\section{ANL-ZPR-491}

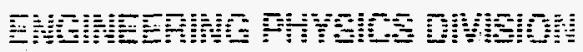

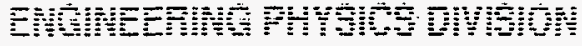

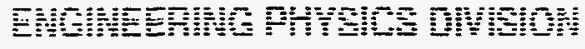

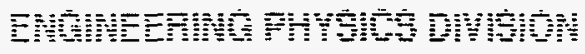

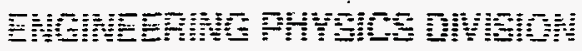
ENGINEERING PHYSICS DIVISION ENGINEERING PHYSICS DIVISION ENGINEERING PHYSICS DIVISION ENGINEERING PHYSICS DIVISION ENGINEERING PHYSICS DIVISION ENGINEERING PHYSICS DIVISION

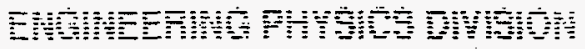

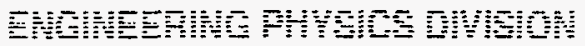

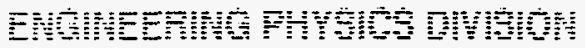

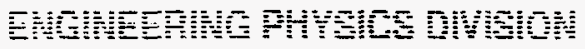

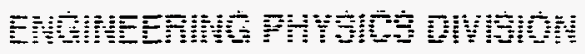

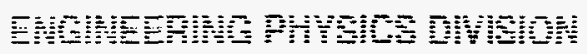

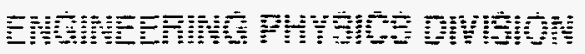

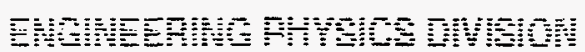

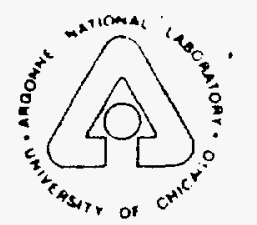

Argonne National Laboratory-West, Idaho Falls, Idaho 83403-2528

Operated by The University of Chicago

for the United States Department of Energy Under Contract W-31-109-Eng-38

$$
\text { and }
$$

AUG 311989

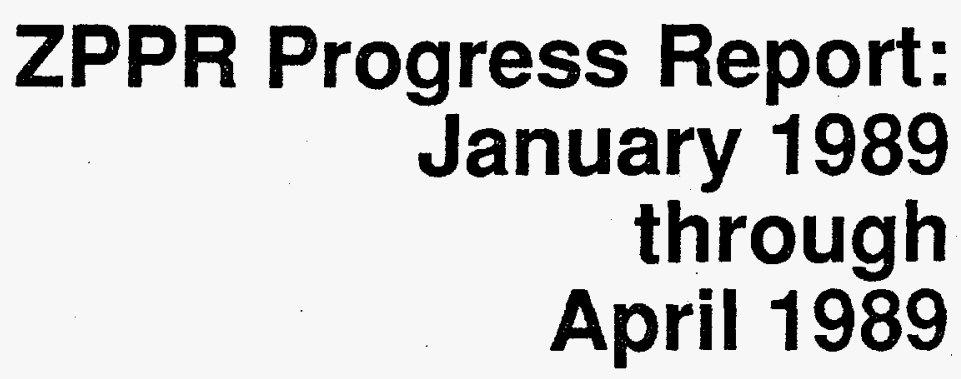

CONTENTS:

ZPPR-18 Control Rod Worths.

ZPPR-18B Reaction Rates.

ZPPR-18 Gamma Dose.

ZPPR-19 Calculation Models.

ZPPR-19 k-effective.

ZPPR-19B Sodium Worth.

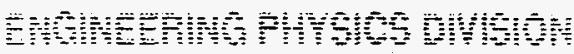

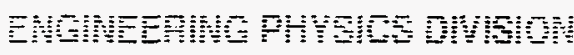

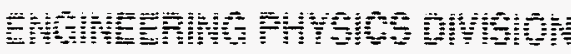

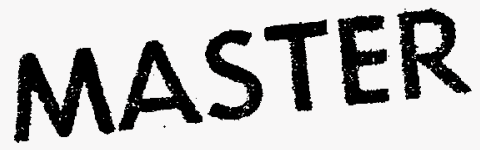

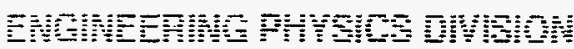

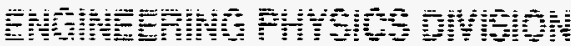


Argonne National Laboratory, with facilities in the states of Illinois and Idaho, is owned by the United States government, and operated by The University of Chicago under the provisions of a contract with the Department of Energy.

\section{DISCLAIMER}

This report was prepared as an account of work sponsored by an agency of the United States Government. Neither the United States Government nor any agency thereof, nor any of their employees, makes any warranty, express or implied, or assumes any legal liability or responsibility for the accuracy, completeness, or usefulness of any information, apparatus, product, or process disclosed, or represents that its use would not infringe privately owned rights. Reference herein to any specific commercial product. process, or service by trade name, trademark manufacturer, or otherwise, does not necessarily constitute or imply its endorsement, recommendation, or favoring by the United States Government or any agency thereof. The views and opinions of authors expressed herein do not necessarily state or reflect those of the United States Government or any agency thereof. 


\title{
ZPPR PROGRESS REPORT: JANUARY 1989 THROUGH APRIL 1989
}

\author{
edited by
}

\author{
P. J. Collins and S. B. Brumbach
}

\author{
Engineering Physics Division \\ Argonne National Laboratory - West \\ P.O. Box 2528 \\ Idaho Falls, ID $\mathbf{8 3 4 0 3 - 2 5 2 8}$
}

\section{DISCLAIMER} This report was prepared as an account of work sponsored by an agency of the United States
Government. Neither the United States Government nor any agency thereof, nor any of their
employees, makes any warranty, express or implied, or as bility for the accuracy, completeness, or usefulned, or assumes any legal liability or responsiprocess disclosed, or represents that its use would of any information, apparatus, product, or ence herein to any specific commercial product pot infringe privately owned rights. Refermanufacturer, or otherwise does not necessarily process, or service by trade name, trademark, mendation, or favoring by the United States Gonstitute or imply its endorsement, recomand opinions of authors expressed herein Government or any agency thereof. The views United States Government ar any agency thereof. 
1. PROGRAM STATUS (S. B. Brumbach and P. J. Collins 1

2. MEASUREMENT AND CALCULATION OF CONTROL ROD WORTHS IN ZPPR-18A (D. M. Smith, P. J. Collins, G. L. Grasseschi and F. Nakashima).

3. THE CONTROL ROD WORTH MEASUREMENT N ZPPR-18B

(F. Nakashima and P. J. Collins)

4. MEASUREMENTS AND ANLAYSIS OF REACTION RATES IN ZPPR-18B (P. J. Collins, J. M. Gasidlo, D. W. Maddison and G. L. Grasseschi)

5. BASIC DATA FOR REACTION RATE DISTRIBUTIONS IN ZPPR-18A AND ZPPR-18B (D. W. Maddison and

J. M. GasidIo)

6. IN-CELL REACTION RATE MEASUREMENTS AND CELL FACTORS FOR 2PPR-18A AND ZPPR-18B (J. M. GasidIo and D. W. Maddison)

7. GAMMA RAY DOSE MEASUREMENTS IN ZPPR-18A AND ZPPR-18B

8. CALCULATION MODELS FOR ZPPR-19A

(G. L. Grasseschi and P.J. Collins)

9. CALCULATION MODELS FOR ZPPR-19B

(G. L. Grasseschi and P.J. Collins)

10. CALCULATED K-EFFECTIVES, DELAYED NEUTRON PARAMETERS

AND ${ }^{241}$ PU DECAY COEFFICIENT FOR ZPPR-19A AND

ZPPR-19B (G. L. Grassesch1 and P. J. Col1ins)

11. SODIUM REACTIVITY WORTHI IN ZPPR-19B (R. W. GOIn)

\title{
DISCLAIMER
}

\begin{abstract}
This report was prepared as an account of work sponsored by an agency of the United States Government. Neither the United States Government nor any agency thereof, nor any of their employees, makes any warranty, express or implied, or assumes any legal liability or responsibility for the accuracy, completeness, or usefulness of any information, apparatus, product, or process disclosed, or represents that its use would not infringe privately owned rights. Reference herein to any specific commercial product, process, or service by trade name, trademark, manufacturer, or otherwise does not necessarily constitute or imply its endorsement, recommendation, or favoring by the United States Government or any agency thereof. The views and opinions of authors expressed herein do not necessarily state or reflect those of the United States Government or any agency thereof.
\end{abstract}




\section{DISCLAIMER}

Portions of this document may be illegible in electronic image products. Images are produced from the best available original document. 


\section{ZPPR PROGRESS REPORT: JANUARY 1989 THROUGH APRIL 1989}

edited by

P. J. Collins and S. B. Brumbach

\section{ABSTRACT}

Further results are presented from the large, homogeneous assembly ZPPR-18 in the JUPITER-III program. Reaction rate results are given for ZPPR-18B along with measured gamma ray dose results from ZPPR-18A and 18B. Control rod worth results from the ZPPR-18 assemblies are included.

Calculation models, measured and calculated k-effective values and measured sodium worth values are presented for the ZPPR-19 assemblies of the lo program. 
1. PROGRAM STATUS (P. J. Collins and S. B. Brumbach)

Processing of experimental data and analysis has been completed for the large conventional cores of the JUPITER-III program, ZPPR-18 and ZPPR-19. This report contains data for control rod worths in $Z P P R-18$, reaction rates in $Z P P R-18 B$, gamma ray dose measurements in $Z P P R-18$ and sodium void reactivities in $Z P P R-19 B$. The data remaining to be reported are reaction rates in $Z P P R-18 C$ and $Z P P R-19 B$ and control rod worths in $Z P P R-19 B$. These will be included in the next report (ANL-ZPR-492) which is in production at this time.

Experimental data for reactivities and reaction rates in mockups of the nuclear assembly test (ZPPR-20A, 20B and 20F) and the reference flight system (ZPPR-20C) have been issued as "ZPPR-20 Data Sheets". Processing of the reactivities for the flooding experiment ( $Z P P R-20 D$ ) and for the sandburied experiment (ZPPR-20E) are nearing completion.

A major concern arising from the initial analysis of ZPPR-20 is the underprediction of control rod reactivity worths. Calculations by the MCNP Monte Carlo code give worths lower than experiment by $16 \%$. The experimental worths are directly proportional to the effective source ratio which, in the case of ZPPR-20, must be calculated. A number of transport calculations using the TWODANT code have been made to investigate errors in the source ratios calculated by diffusion theory in xyz geometry. Differences of only $1-2 \%$ are indicated for a range of cases. The $r z$ model, with a suitable shielding factor derived for the control rod annulus gives excellent agreement with MCNP results for the rod worths.

A processing code which constructs the input data for the VIM Monte Carlo code directly from the ZPPR experimental data base is operational. The ZPPR assemblies are described in great detail including drawers, cladding of the material plates and air gaps. Initial tests indicate that a value of the source ratio will be obtained to useful accuracy from the Monte Carlo calculations. 
2. MEASUREMENT AND CALCULATION OF CONTROL ROD WORTHS IN ZPPR-18A

(D. M. Smith, P. J. Collins, G. L. Grasseschi and F. Nakashima*)

\subsection{Introduction}

The ZPPR-18 assembly was designed to provide basic physics data for a very large, homogeneous LMR. Of particular interest were spatially sensitive parameters, including control rod worths. The ZPPR-18A configuration contained 24 control rod positions (CRPs) arranged as an inner ring of six, a middle ring of six and an outer ring of twelve. The outer bank is located at the boundary between the inner core and outer core. Because there were distinct zones of plutonium and uranium fuel in the outer core, outer ring CRPs are not all in equivalent environments.

In addition to measurements in the standard CRP locations, the worths of individual rods and pairs of rods were measured as a function of radius along the $x$-axis of the assembly. These latter measurements were made with respect to fuel. The control locations are given in Fig. 2.1. As shown, all CRPs are two-by-three drawers in size except for the special measurements at $x$-axis locations in the inner core which are three-by-three drawers in the inner core.

The measurements fall into four basic categories; those with $100 \%$ natural $B_{4} C$ rods, those at various radil along the $x$-axis, banks and partial banks with $50 \%$ natural $B_{4} C$ rods and half-inserted banks. All measurements were made in the ZPPR-18A subcritical reference (described in ANL-ZPR-485, p 2). This reference loading was rebuilt immediately prior to the control rod experiments, after the $\mathrm{x}$-axis measurements and after the $50 \% \mathrm{~B}_{4} \mathrm{C}$ rod bank measurements. The reactivity was established by inverse kinetics analysis of the power history following a rod drop. The experimental rod worths were measured relative to this reference reactivity by the modifiedsource-multiplication method using the countrates recorded on a system of

* On assignment from the Power Reactor and Nuclear Fuel Development Corporation. 
sixty-four in-core fission chambers. All measured values presented here are from the least-squares fitting of reactivity versus detector efficiency in the McCRUNCH code. Adjustments to the calculated effective source ratio after the least squares fitting were small, usually of the order of tenths of a percent and about $1 \%$ for some multiple rod patterns.

Various control rod drawers were used. Atom densities corresponding to these control rod and CRP drawers, with axial sections labeled in inches, are given in Table 2.1 .

\subsection{Measurements with $100 \% \mathrm{~B}_{4} \mathrm{C}$ Rods}

The worth of each of the three CR banks was measured for rods containing $100 \%$ natural $\mathrm{B}_{4} \mathrm{C}$. Master 603 was used in rods 1 to 6,7 to 12 and $13,14,17,18,19,20,23$ and 24. Master 604 was used in rods 15, 16, 21 and 22. Details of the data processing are given in Table 2.2. The ref erence reactivity prior to the measurements (loading number 45) was $-22.92 \phi \pm 0.18 \phi$. The 64 detector data for this reference were recorded on data file 42.

\subsection{Measurements of Worth vs Radius on the $x^{\text {taxis }}$}

A series of thirteen worth measurements was made for single rods and rod pairs along the x-axis. These measurements were made with respect to fuel. Later, the worths of CRPs were measured in the central location (A) and an outer location $(E+f)$. For all locations, the $50 \% \mathrm{~B}_{4} \mathrm{C}$ master 606 was used. The reference reactivity was determined at the end of this measurement sequence and was $-25.81 \notin \pm 0.21 \notin$ (loading number 62,64 mcounter data file 59, inverse kinetios file 43). After correcting for temperature, interface gap and ${ }^{241} \mathrm{Pu}$ decay, the difference between reference reactivities in loadings 45 and 62 was $0.30 \phi$. This difference is attributed to material movement in loading and unloading fuel drawers during the $x$-axis measure ments. Details of the data processing are given in Table 2.3. 
2.4 Worths of Banks and Partial Banks with $50 \% \mathrm{~B}_{4} \mathrm{C}$ Rods

Worths were measured for twentyiseven conflgurations which included unique individual rods, whole banks, partial banks and combinations of banks.

The control rod drawer masters assigned to the various locations are given in Table 2.4. The reference reactivity for this measurement sequence was that described above and measured in loading $62(-25.81 \phi)$. A reference reactivity measurement was repeated after this serles of measurements and differed from the loading 62 reactivity by only $0.03 \phi$, and continued use of the previous reference is appropriate.

Details of the data processing are given in Table 2.4.

\subsection{Worths of Half-Inserted Rod Banks}

Worths were also measured for a few configurations with halfinserted rods. All half-inserted rods had their $B_{4} C$ in half two of the assembly. The drawers used for half-inserted rods were master 608 and contained $787 \mathrm{~mm}$-long (31-inch) columns of natural $\mathrm{B}_{4} \mathrm{C}$ in contrast to $508 \mathrm{~mm}$ iong (20 inch) columns in drawers used for fully inserted rods. Master 605 was used in CR 24 when CR 24 was fully inserted. The reference reactivity for this measurement is again that from loading $62(-25.81 \phi)$. Details of the data processing are given in Table 2.5.

\subsection{Reference Diffusion Calculations and Comparisons with Measurement}

The basic calculations were required to provide adjoint fluxes and sourcerdriven real fluxes for each control rod measurement in order to calculate detector efficiencies and effective source ratios for processing the experimental data. For economy, these calculations were made with diffusion theory in $x y$ geometry with cross sections collapsed to six energy groups. The adjoint k-effectives give calculated worths for each case. 
This simple calculation model gave excellent predictions of flux distributions relative to those in the reference core. This can be seen in Tables 2.4 and 2.5 where the number of detectors retained in the MCCRUNCH processing was close to 60 in almost all cases. The good predictions of detector efficiencies give confidence in the calculated effective source ratios.

The calculations were made with the finite difference path of DIF3D for convenience in the editing codes (local to ZPPR usage) rather than the nodal diffusion path. A mesh spacing of $55 \mathrm{~mm}$ (one mesh per ZPPR matrix position) was used. Thus calculated rod worths are closer to transport results than by the nodal method due to mesh size errors.

The six group data were collapsed from an xyz model of the reference core calculated in 21 groups. This model used average compositions for each characteristic drawer master type - In the inner core (single plutonium column types DUM and DUF), in the outer core (plutonium single and double column, uranium single and double column), control positions (CRPs), and each blanket and reflector zone. A second xyz calculation with all even numbered controls rods inserted was used to collapse data for control rods. Data were collapsed individually for each rod in the ring. Thus, in the outer ring, the control rods adjacent to the plutonium section (Numbers 24 and 18) were distinct from those near the uranium section (Numbers 22 and 16). However, cross sections for the odd numbered rods used data collapsed for the even numbered rods.

Effective buckling terms for each drawer type in the reference and for control rods were generated by a method analogous to that used for group collapse. Two xyz calculations in six groups provided axial leakages at the core/axial blanket interface which were matched to group and region dependent $\mathrm{DB}^{2}$ terms.

Benoist diffusion coefficient modifiers were collapsed from 21 groups to 6 groups using 


$$
\sum D_{1 g} \Phi_{g} / \Sigma D_{\text {homg }} \Phi_{g}
$$

with $i=x, y, z, D_{\text {homg }}$ the Isotropic diffusion coefficient and summation over the 6 group subsets of the 21 groups.

Although the six group collapse was done with generic master compositions, the $x y$ models used the individual drawer compositions in the loading as for the $x y z$ calculation models described in ANL ZPR $-489, p .12$ et. seq.

Calculated worths are defined as $\left(k_{1}-k_{2}\right) /\left(k_{1} k_{2} \beta\right)$ with $k_{1}$ the $k$-effective for the subcritical reference, $k_{2}$ the k-effective for the case with control rods inserted and $\beta=0.0037855$, the calculated $\beta$ teffective for ZPPR-18A (ANL-ZPR-489, p. 49). The kreffective calculated for the subcritical reference $x y$ model was 0.989333 .

Five measurements were made for patterns of half-inserted control rods (Table 2.5). In the $x y$ models these rods were represented with control rods and CRP compositions and bucklings combined with 50\% volume fractions. The MCCRUNCH processing was carried out in two steps. First, the processing was done with the halfone detectors and half-two detectors separately. This gave average biases of 1.023 for the reactivity predicted by the halftwo detectors and 0.977 for the halfrone detectors compared with the mean of all detectors. In the second stage, the efficiencies for half two detectors were adjusted by a factor 0.977 and those for the halfrone detectors by 1.023. This produced an adequate fit to the experiment countrates with over 50 detectors being retained in the data processing (Table 2.5). The calculated rod worths were overpredicted by about $5 \%$ relative to the results for the rods fully inserted due to underestimation of boron shlelding with the 50\% density rods in the model. Threemdimensional calculations will be needed to improve these results.

\subsubsection{Control Rods Along the X-axis}

A series of control rod worth measurements was made for positions along the $x$-axis to provide detail on the accuracy of prediction 
of worths as a function of radius and to give rod interaction effects. The rods occupied $3 \times 3$ matrix positions in the inner core and $2 \times 3$ matrix positions in the outer core (Figure 2.1). The rod worths were measured relts ative to fuel rather than relative to CRPs as in the other measurements. A similar series of measurements was made in the smaller conventional core ZPPR -9 (ZPR-TM-356 p. 2). A comparison of the measured and calculated worths for ZPPR-18A is shown in Table 2.6 and interaction effects are given in Table 2.7 .

The results of the analysis are:

(i) The rod worths are predicted most consistently with a mean $\mathrm{C} / \mathrm{E}$ of 1.104 and standard deviation 0.006 . No trend in $\mathrm{C} / \mathrm{E}$ with radius is apparent, including rod. $G$ which is in the steep flux gradient at the edge of the outer core. The unvarying prediction is consistent with the analysis of fission rate distributions (ANL-ZPR-489) and contrasts with results in the smaller conventional cores of the Jupiter-I series.

(ii) The worths of CRPs relative to fuel are overpredicted by $20 \%$. A large overprediction is always obtained by diffusion calculations and has been shown to be due to overprediction of axial leakage in the sodium channels. Note that in the present case the axial leakage errors are contained in the CRP buckling terms which were calculated from an xyz diffusion model.

(iii) The C/Es for control rod worths relative to fuel are higher than those relative to CRPs by about $3 \%$. This is shown in the last two lines of Table 2.6 where experimental and calculated worths are given relative to CRPs. All other control rod worths in ZPPR-18A were made In the CRP positions of the reference loading. As will be seen, the $C / E$ results relative to CRPs of 1.064 ( $C R A$ ) and 1.0770 (CRs $F+f$ ) are consistent with other results for inner $r$ ing rods and outer $r$ ing rods near the plutonium sector.

(iv) Interaction effects are usually defined as the percent change in the combined rod worth relative to the sum of the 
individual worths. These interactions are well calculated by the simple diffusion model (table 2.7). The interaction effects for ZPPR 18A in percent appear to be smaller than in ZPPR-9. For example, the interaction for the pair of outer ring control rods relative to fuel in ZPPR-9 (rods 13, 19 in ZPPR -9 numbering scheme) is $27 \%$ while that for rods $E+e$ in $Z P P R-18 B$ is 17\%. However, a better comparison is made by normalizing the interaction percent to the worth of a single rod, as is shown in the fourth column of Table 2.7. Since the rods in $Z P P R-9$ have about twice the worth of those in ZPPR-18A, the normalized interactions for the pair of outer $r i n g$ rods are $15 \% \$^{-1}$ in ZPPR -9 and $21 \% \$^{-1}$ in ZPPR-18A.

\subsubsection{Worths of Control Rod Banks and Constituent Rods}

These data have been selected to show the analysis of the principal rod banks and of single rods, pairs of rods, and quartets of rods in the banks. The single rods were measured in each of the positions with $60^{\circ}$ symmetry in the banks. Sets of two and four rods were measured at positions in the outer bank adjacent to the plutonium sector and adjacent to the uranium sector to provide data on the azimuthal predictions in the outer ring which could be calculated by refined methods using one-eighth core symmetry. Differences in accuracy of prediction of rods near the two sectors have an impact on the utilization of the complete rod bank worths for an all-plutonium fueled core.

Results in the following tables are given a descriptive label for ease of comprehension. The full description of the measurement can be found in Tables 2.3 to 2.5 by means of the data file numbers. Single rod positions are indicated by the angle relative to the positive $x$-axis. Note that the statistical uncertainties for the measurement are between $0.1 \%$ and $0.2 \%$. This is the standard by which to compare the relative accuracies of predictions.

The analysis of rods in the individual banks is given in Table 2.8. A number of interesting conclusions result: 
(i) The rod worths in the inner ring are predicted with remarkable consistency. The $100 \% \mathrm{~B}_{4} \mathrm{C}$ rod bank has a $\mathrm{C} / \mathrm{E}$ which is marginally higher than the standard $50 / 50 \mathrm{~B}_{4} \mathrm{C} / \mathrm{Na}$ rod.

(ii) In the middle ring, $C R 11$ at the $y$-axis has a $C / E$ lower than that of CR 12 near the $x$-axis by $1.8 \%$. This difference is attributed to underpredictions of fluxes near the uranium sector relative to prediction near the plutonium sector.

(iii) In the outer ring, rods adjacent to the uranium sector have a C/E lower by $5 \%$ to $6 \%$ than those adjacent to the plutonium section (compare results for $C R 22$ and $C R 24$, pairs of rods and quartets of rods). This difference is consistent with analysis of fission rates at the $y$ - and $x$ - axes (ANL-ZPR-489, p. 50). The average of the $C / E s$ for rods 21 , 23, and 24 is 1.042 and consistent with the C/Es for 12 outer ring rods (1.041) and for 6 outer ring rods (1.039). This result shows that the $\mathrm{C} / \mathrm{E}$ bias for the banks (1.04) should not be applied to an all plutonium core. However, the consistent $\mathrm{C} / \mathrm{Es}$ for the banks and single rods show that the bias factors could be taken from the results of measurements near the plutonium sectors.

(iv) The difference of $1.5 \%$ between C/Es for CRs 15 and 16 and for rods $15,16,21$, and 22 is evidence of a tilt between the top and bottom of the ZPPR matrix. As shown in detail in ZPPR-13 experiments, the reactor halves are in contact at the top of the assembly and separated by about $1 \mathrm{~mm}$ at the bottom. Control rods 15 and 16 were positioned at the lower part of the assembly where fuel is separated and measured worths are thus lower than at the top. The difference between CRs 15 and 16 and CRs 21 and 22 was measured in $Z P P R-19 B$.

(v) The worths of the banks of $100 \% \mathrm{~B}_{4} \mathrm{C}$ rods are calculated with biases similar to those for the $50 \% \mathrm{~B}_{4} \mathrm{C}$ rod banks (Table 2.8). 


\subsubsection{Rod Bank Combinations and Interactions}

Three measurements were made of combinations of complete control rod banks. The results are given in Table 2.9. The worths of the individual rod banks from Table 2.8 are also included for comparison.

Small negative interactions are found for adjacent rod banks, $-2 \%$ for $I R+M R$ and $-4 \%$ for $M R+O R$. A large interaction of $32 \%$ occurs between the more widely spaced inner and outer banks. In this case, the worth of inserting the inner bank when the outer bank is already inserted in the core is $\$ 11.28$ which is $58 \%$ greater than the worth of the inner bank alone.

These interaction effects are adequately predicted by the simple diffusion calculations.

\subsubsection{Rod Bank Combinations with Missing Rods}

Measurements were made for combinations of two rod banks with a rod missing from each bank in turn. The missing rod in each case was one nearest to the plutonium sectors. These results are given in Table 2.10. The results for the complete rod banks (Table 2.9) are also included for comparison. The $\mathrm{C} / \mathrm{E}$ values for each of the three sets are consistent within $0.4 \%$ but these results are not a sensitive test of calculation.

By subtracting these results one can deduce the worth of the missing control rods. This is important in evaluation of rod run-out or stuck rod effects. This analysis is given in Table 2.11. Comparisons are made with the single rod worth and the worth of the rod within its bank. Note that the statistical uncertainties, which are shown in the table, may be relatively large due to subtraction of two worths of similar magnitude.

The worths of single rods vary considerably due to interaction effects between rods in a ring and interaction effects between rod banks. The worth of a rod in the inner $r i n g$ is reduced in comparison 
with the isolated rod due to a negative interaction between rods in the ring and further reduced if the middle ring is also inserted. However, the worth is increased by $60 \%$ when the outer bank rods are inserted.

A strong positive interaction exists in the middle ring because of the wide separation of the rods and the single rod worth is enhanced by $77 \%$. The worth is further enhanced to about a factor of 2 greater when the inner ring bank is present but the increase in worth is only $46 \%$ when the neighboring outer bank is inserted. Interaction effects in the outer ring gave an increase in the single rod worth of $12 \%$ but insertion of the inner ring enhances the single rod worth by $180 \%$.

These effects are quite well calculated as can be seen by comparing the relative $\mathrm{C} / \mathrm{E}$ results for each rod group.

\subsubsection{Half Inserted Rods}

The present calculations are almed at data reduction and do not provide good comparisons with experiment. Improvements would require threedimensional calculations or derivation of appropriate rod shielding factors and buckling terms for use in the xy model. For completeness, however, the results for these cases are recorded in Table 2.12.

The consistency among the C/E results in Table 2.12 is quite remarkable, although the sample model results in biases which are 6-9\% higher than those for the fully inserted rods.

The worth of control rod 24 is $0.784 \$$ with 6 inner ring and 11 outer ring rods half inserted (Table 2.5, Files 93 and 94). This compares with the worth of $1.479 \$$ with the same rods fully inserted and the worth of $0.514 \$$ when inserted alone (Table 2.11).

\subsubsection{Threerdimensional Diffusion and Transport Calculations}

A series of three-dimensional calculations with 21 group cross sections was made for selected cases exhibiting one-eighth core 
symmetry in order to provide refined results for comparison with data in other cores. The cases chosen were the three complete rod banks, both with $100 \% \mathrm{~B}_{4} \mathrm{C}$ rods and with the $50 / 50 \mathrm{~B}_{4} \mathrm{C} / \mathrm{Na}$ reference rods, plus the two groups of four rods adjacent to the plutonium sector and adjacent to the uranium sector. The latter cases were of particular interest because of the different accuracies of prediction found for rods near the two sectors. These differences clearly have an impact on the use of the outer rod bank with analysis for extrapolation to an all plutonium fueled core. Most effective use of the data may require a full sensitivity analyses.

Three dimension diffusion calculations were made using the nodal diffusion and nodal transport solutions. The $x y$ mesh in the nodal solutions was constrained by the ZPPR geometry to $55 \mathrm{~mm}$ but coarse node spacings of 100-150 mm were used in the $z$ dimension. The k-effective values are recorded in Table 2.13 and Table 2.14 compares the C/E results for the two methods.

Transport corrections, relative to the nodal diffusion, are close to $-3 \%$ for the inner and middle ring rods and $-1.5 \%$ for the outer ring rod bank. Different transport corrections are calculated for the outer ring rods adjacent to the plutonium sector $(-0.8 \%)$ and for the rods adjacent to the uranium sector $(-2.5 \%)$. The simple $x y$ calculations give results within about $2 \%$ of those from the $x y z$ transport values. This result is typical of the compensation of course mesh and transport errors found in conventional cores, but effects of group collapse and axial buckling approximations have not been explored for $Z P P R-18$.

The $x y z$ calculations show about $2 \%$ higher $C / E$ values for the $100 \% \mathrm{~B}_{4} \mathrm{C}$ rods than for the reference $\mathrm{B}_{4} \mathrm{C} / \mathrm{Na}$ rods. This is attributed to heterogeneity effects in the $\mathrm{B}_{4} \mathrm{C} / \mathrm{Na}$ rods which has not been treated in the calculations.

The xyz calculation confirms the lower $C / E$ for rods adjacent to the uranium sector than adjacent to the plutonium sector ( $5 \%$ lower by diffusion, $6 \%$ lower by transport). This difference clearly affects the $\mathrm{C} / \mathrm{E}$ for the outer ring bank and possibly the middle ring bank. This 
result appears to be consistent with the analysis of foil reaction rate data. The bias factor developed for an all-plutonium loaded core would need to take account of this effect. 
TABLE 2.1 Atom Densities for the Control Rods in ZPPR-18 (atoms/barn-cm)

\begin{tabular}{|c|c|c|c|c|c|c|}
\hline Isotopes & $\begin{array}{c}\text { Control } \\
\text { Position } \\
\text { Master } 601 \\
0-20 \\
\end{array}$ & $\begin{array}{c}\text { Control } \\
\text { Position } \\
\text { Master } 601 \\
20-31 \\
\end{array}$ & $\begin{array}{c}\text { Control } \\
\text { Position } \\
\text { Master } 601 \\
31-36 \\
\end{array}$ & $\begin{array}{c}\text { Control } \\
\text { Position } \\
\text { Master } 602 \\
0-20 \\
\end{array}$ & $\begin{array}{c}\text { Control } \\
\text { Position } \\
\text { Master } 602 \\
20-31 \\
\end{array}$ & $\begin{array}{c}\text { Control } \\
\text { Position } \\
\text { Master } 602 \\
31-36 \\
\end{array}$ \\
\hline BO & 0.0 & 0.0 & 0.0 & 0.0 & 0.0 & 0.0 \\
\hline B1 & 0.0 & 0.0 & 0.0 & 0.0 & 0.0 & 0.0 \\
\hline $\mathrm{C}$ & 0.0000308 & 0.0000309 & 0.0000447 & 0.0000307 & 0.0000309 & 0.0000447 \\
\hline 0 & 0.0000 & 0.00 & 0.00 & 0.00 & 0.000 & 0.0 \\
\hline $\mathrm{Na}$ & 0.0 & 4405 & 79925 & 0.0 & 0.0 & 0.0 \\
\hline $\mathrm{Si}$ & 0.0001649 & 1649 & 0.0001705 & 0.0001645 & 0.0001649 & 001705 \\
\hline Al & 0.0000047 & 0049 & 0.0000048 & 0.0000047 & 0.0000049 & 0.0000048 \\
\hline Mn & 0.0002433 & 0.0002443 & 0.0002511 & 0.0002430 & 0.0002443 & 0.0002511 \\
\hline $\mathrm{Cr}$ & 0.0029568 & 0.0029657 & 0.0030388 & 0.0029514 & 0.0029657 & 0.0030388 \\
\hline $\mathrm{Fe}$ & 0.0104374 & 0.0104697 & 0.0110018 & 0.0104182 & 0.0104697 & 0.0110018 \\
\hline $\mathrm{Ni}$ & 0.0013247 & 0.0013281 & 0.0013586 & 0.0013223 & 0.0013281 & 0.0013586 \\
\hline $\mathrm{Cu}$ & 0.0000354 & 0.0000353 & 0.0000370 & 0.0000353 & 0.0000353 & 0.0000370 \\
\hline Mo. & 0.0000174 & 0.0000174 & 0.0000183 & 0.0000173 & 0.0000174 & 0.0000183 \\
\hline$P$ & 0.0000040 & 0.0000040 & 0.0000042 & 0.0000040 & 0.0000040 & 0.0000042 \\
\hline$S$ & 0.0000012 & 0.0000012 & 0.0000013 & 0.0000012 & 0.0000012 & 0.0000013 \\
\hline $\mathrm{Cl}$ & 0.0000006 & 0.0000006 & 0.0000006 & 0.0000006 & 0.0000006 & 0.0000006 \\
\hline $\mathrm{Ca}$ & 0.0000042 & 0.0000042 & 0.0000041 & 0.0000042 & 0.0000042 & 0.0000041 \\
\hline $\mathrm{Co}$ & 0.0000038 & 0.0000037 & 0.0000043 & 0.0000038 & 0.0000037 & 0.0000043 \\
\hline
\end{tabular}


TABLE 2.1 (Contd)

\begin{tabular}{|c|c|c|c|c|c|c|}
\hline Isotopes & $\begin{array}{c}\text { Control } \\
\text { Rod } \\
\text { Master } 603 \\
0-20 \\
\end{array}$ & $\begin{array}{c}\text { Control } \\
\text { Rod } \\
\text { Master } 603 \\
20-31 \\
\end{array}$ & $\begin{array}{c}\text { Control } \\
\text { Rod } \\
\text { Master } 603 \\
31+36 \\
\end{array}$ & $\begin{array}{c}\text { Control } \\
\text { Rod } \\
\text { Master } 604 \\
0-20 \\
\end{array}$ & $\begin{array}{c}\text { Control } \\
\text { Rod } \\
\text { Master } 604 \\
20-31 \\
\end{array}$ & $\begin{array}{c}\text { Control } \\
\text { Rod } \\
\text { Master } 604 \\
31-36 \\
\end{array}$ \\
\hline BO & 0.0153857 & 0.0 & 0.0 & 0.0152058 & 0.0 & 0.0 \\
\hline $\mathrm{B} 1$ & 0.0623918 & 0.0 & 0.0 & 0.0616654 & 0.0 & 0.0 \\
\hline $\mathrm{C}$ & 0.0199484 & 0.0000309 & 0.0000447 & 0.0098260 & 0.0000309 & 0.0000447 \\
\hline 0 & 0.0000781 & 0.00000 & 0.0000 & 0.0000651 & 0.0000013 & 0.0000013 \\
\hline $\mathrm{Na}$ & 0.0 & 0.0181363 & 0.0 & 0.0 & 0.0181363 & 0.0179925 \\
\hline Si & 0.0002321 & 0.0001651 & 1705 & 0.000 & 0.0001651 & 0.0001705 \\
\hline AI & 0.0000026 & 0.0000043 & 0.00 & 0.00 & 0.0000043 & 0.0000048 \\
\hline $\mathrm{Mn}$ & 0.0002144 & 0.0002432 & 0.0002512 & 0.00 & 0.0002432 & 0.0002512 \\
\hline $\mathrm{Cr}$ & 0.0025322 & 0.0029562 & 0.0030388 & 0.0022743 & 0.0029562 & 0.0030388 \\
\hline $\mathrm{Fe}$ & 0.0090962 & 0.0104363 & 0.0110018 & 0.0082314 & 0.0104363 & 0.0110018 \\
\hline $\mathrm{Ni}$ & 0.0011231 & 0.0013233 & 0.0013586 & 0.0009914 & 0.0013233 & 0.0013586 \\
\hline $\mathrm{Cu}$ & 0.0000322 & 0.0000357 & 0.0000370 & 0.000 & 0.0000357 & 0.0000370 \\
\hline Mo & 0.0000161 & 0.0000174 & 0.0000183 & 0.0000155 & 0.0000174 & 0.0000183 \\
\hline$P$ & 0.0000040 & 0.0000040 & 0.0000042 & 0.0000040 & 0.0000040 & 0.0000042 \\
\hline$S$ & 0.0000012 & 0.0000012 & 0.0000013 & 0.0000012 & 0.0000012 & 0.0000013 \\
\hline $\mathrm{Cl}$ & 0.0 & 0.0000006 & 0.0000006 & 0.0 & 0.0000006 & 0.0000006 \\
\hline $\mathrm{Ca}$ & 0.0 & 0.0000042 & 0.0000041 & 0.0 & 0.0000042 & 0.0000041 \\
\hline Co & 0.0000038 & 0.0000037 & 0.0000043 & 0.0000038 & 0.0000037 & 0.0000043 \\
\hline
\end{tabular}


TABLE 2.1 (Contd)

\begin{tabular}{|c|c|c|c|c|c|c|}
\hline Isotopes & $\begin{array}{c}\text { Control } \\
\text { Rod } \\
\text { Master } 605 \\
0-20 \\
\end{array}$ & $\begin{array}{c}\text { Control } \\
\text { Rod } \\
\text { Master } 605 \\
20-31 \\
\end{array}$ & $\begin{array}{c}\text { Control } \\
\text { Rod } \\
\text { Master } 605 \\
31-36 \\
\end{array}$ & $\begin{array}{c}\text { Control } \\
\text { Rod } \\
\text { Master } 606 \\
0-20 \\
\end{array}$ & $\begin{array}{c}\text { Control } \\
\text { Rod } \\
\text { Master } 606 \\
20-31 \\
\end{array}$ & $\begin{array}{c}\text { Control } \\
\text { Rod } \\
\text { Master } 606 \\
31-36 \\
\end{array}$ \\
\hline BO & 0.0071583 & 0.0 & 0.0 & 0.0085434 & 0.0 & 0.0 \\
\hline B1 & 0.0290256 & 0.0 & 0.0 & 0.0346512 & 0.0 & 0.0 \\
\hline $\mathrm{C}$ & 0.0094051 & 0.0000309 & 0.0000447 & 0.0110453 & 0.0000309 & 0.0000447 \\
\hline 0 & 0.0000006 & 0.0000013 & 0.0000013 & 0.0000787 & 0.0000013 & 0.0000013 \\
\hline $\mathrm{Na}$ & 0.0087470 & 0.0181363 & 0.0179925 & 0.0087470 & 0.0181363 & 0.0179925 \\
\hline $\mathrm{Si}$ & 0.0002035 & 0.0001651 & 0.0001705 & 0.0001999 & 0.0001651 & 0.0001705 \\
\hline $\mathrm{Al}$ & 0.0000054 & 0.0000043 & 0.0000048 & 0.0000033 & 0.0000043 & 0.0000048 \\
\hline $\mathrm{Mn}$ & 0.0002605 & 0.0002432 & 0.0002512 & 0.0002198 & 0.0002432 & 0.0002512 \\
\hline $\mathrm{Cr}$ & 0.0031765 & 0.0029562 & 0.0030388 & 0.0026312 & 0.0029562 & 0.0030388 \\
\hline $\mathrm{Fe}$ & 0.0112393 & 0.0104363 & 0.0110018 & 0.0094141 & 0.0104363 & 0.0110018 \\
\hline $\mathrm{Ni}$ & 0.0014421 & 0.0013233 & 0.0013586 & 0.0011628 & 0.0013233 & 0.0013586 \\
\hline $\mathrm{Cu}$ & 0.0000361 & 0.0000357 & 0.0000370 & 0.0000336 & 0.0000357 & 0.0000370 \\
\hline Mo & 0.0000178 & 0.0000174 & 0.0000183 & 0.0000167 & 0.0000174 & 0.0000183 \\
\hline$P$ & 0.0000040 & 0.0000040 & 0.0000042 & 0.0000040 & 0.0000040 & 0.0000042 \\
\hline $\mathrm{S}$ & 0.0000012 & 0.0000012 & 0.0000013 & 0.0000012 & 0.0000012 & 0.0000013 \\
\hline $\mathrm{Cl}$ & 0.0000003 & 0.0000006 & 0.0000006 & 0.0000003 & 0.0000006 & 0.0000006 \\
\hline $\mathrm{Ca}$ & 0.0000020 & 0.0000042 & 0.0000041 & 0.0000020 & 0.0000042 & 0.0000041 \\
\hline Co & 0.0000038 & 0.0000037 & 0.0000043 & 0.0000038 & 0.0000037 & 0.0000043 \\
\hline
\end{tabular}


TABLE 2.1 (Contd)

\begin{tabular}{|c|c|c|c|c|c|c|}
\hline Isotopes & $\begin{array}{c}\text { Control } \\
\text { Rod } \\
\text { Master } 607 \\
0-20 \\
\end{array}$ & $\begin{array}{c}\text { Control } \\
\text { Rod } \\
\text { Master } 607 \\
20 \div 31 \\
\end{array}$ & $\begin{array}{c}\text { Control } \\
\text { Rod } \\
\text { Master } 607 \\
31-36 \\
\end{array}$ & $\begin{array}{c}\text { Control } \\
\text { Rod } \\
\text { Master } 608 \\
0-20 \\
\end{array}$ & $\begin{array}{c}\text { Control } \\
\text { Rod } \\
\text { Master } 608 \\
20-31 \\
\end{array}$ & $\begin{array}{c}\text { Control } \\
\text { Rod } \\
\text { Master } 608 \\
31-36 \\
\end{array}$ \\
\hline BO & 0.0071436 & 0.0 & 0.0 & 0.0071583 & 0.0072759 & 0.0 \\
\hline B1 & 0.0289671 & 0.0 & 0.0 & 0.0290256 & 0.0295024 & 0.0 \\
\hline$c$ & 0.0093870 & 0.0000309 & 0.0000447 & 0.0094051 & 0.0095480 & 0.0000479 \\
\hline 0 & 0.0000006 & 0.0000013 & 0.0000013 & 0.0000006 & 0.0000068 & 0.0000013 \\
\hline $\mathrm{Na}$ & 0.0087470 & 0.0181363 & 0.0179925 & 0.0087470 & 0.0087131 & 0.0175023 \\
\hline Si & 0.0002043 & 0.0001651 & 0.0001705 & 0.0002035 & 0.0002034 & 0.0001905 \\
\hline$A 1$ & 0.0000051 & 0.0000043 & 0.0000048 & 0.0000054 & 0.0000048 & 0.0000060 \\
\hline Mn & 0.0002613 & 0.0002432 & 0.0002512 & 0.0002605 & 0.0002578 & 0.0002692 \\
\hline $\mathrm{Cr}$ & 0.0031882 & 0.0029562 & 0.0030388 & 0.0031765 & 0.0031389 & 0.0032725 \\
\hline $\mathrm{Fe}$ & 0.0112792 & 0.0104363 & 0.0110018 & 0.0112393 & 0.0111169 & 0.0118247 \\
\hline $\mathrm{Ni}$ & 0.0014482 & 0.0013233 & 0.0013586 & 0.0014421 & 0.0014216 & 0.0014830 \\
\hline $\mathrm{Cu}$ & 0.0000361 & 0.0000357 & 0.0000370 & 0.0000361 & 0.0000359 & 0.0000384 \\
\hline Mo & 0.0000178 & 0.0000174 & 0.0000183 & 0.0000178 & 0.0000177 & 0.0000189 \\
\hline $\mathrm{P}$ & 0.0000040 & 0.0000040 & 0.0000042 & 0.0000040 & 0.0000040 & 0.0000050 \\
\hline$S$ & 0.0000012 & 0.0000012 & 0.0000013 & 0.0000012 & 0.0000012 & 0.0000013 \\
\hline $\mathrm{Cl}$ & 0.0000003 & 0.0000006 & 0.0000006 & 0.0000003 & 0.0000003 & 0.0000006 \\
\hline $\mathrm{Ca}$ & 0.0000020 & 0.0000042 & 0.0000041 & 0.0000020 & 0.0000020 & 0.0000040 \\
\hline Co & 0.0000038 & 0.0000037 & 0.0000043 & 0.0000038 & 0.0000037 & 0.0000051 \\
\hline
\end{tabular}


TABLE 2.2 Data Processing for ZPPR-18A $100 \%$ Natural B $C$ Control Rod Measurements

\begin{tabular}{|c|c|c|c|c|c|c|c|c|}
\hline $\begin{array}{l}\text { Control } \\
\text { Rods }\end{array}$ & $\begin{array}{l}\text { Reactor } \\
\text { Loading } \\
\text { Number }\end{array}$ & $\begin{array}{r}\text { Data } \\
\text { File } \\
\text { Number } \\
\end{array}$ & $\begin{array}{l}\text { Number } \\
\text { of FCs } \\
\end{array}$ & $x^{2}$ & $\begin{array}{l}\text { Source } \\
\text { Ratio }\end{array}$ & $\begin{array}{l}\text { Measured } \\
\text { Worth, } \$\end{array}$ & $\begin{array}{c}\text { Statistical } \\
\text { Uncertainty, } \%\end{array}$ & $\begin{array}{c}\text { Total } \\
\text { Uncertainty, } \%\end{array}$ \\
\hline $1-6$ & 46 & 43 & 61 & 1.279 & 1.0036 & 8.444 & 0.152 & 0.869 \\
\hline $7-12$ & 47 & 44 & 60 & 1.340 & 0.9851 & 7.997 & 0.136 & 0.868 \\
\hline $13-24$ & 48 & 45 & 61 & 1.472 & 0.8521 & 7.408 & 0.146 & 1.029 \\
\hline
\end{tabular}


TABLE 2.3 Data Processing for Control Rods on the X-Axis in ZPPR-18A

\begin{tabular}{|c|c|c|c|c|c|c|c|c|}
\hline $\begin{array}{l}\text { Control } \\
\text { Rods } \\
\end{array}$ & $\begin{array}{l}\text { Reactor } \\
\text { Loading } \\
\text { Number } \\
\end{array}$ & $\begin{array}{r}\text { Data } \\
\text { File } \\
\text { Number } \\
\end{array}$ & $\begin{array}{l}\text { Number } \\
\text { of FCs } \\
\end{array}$ & $x^{2}$ & $\begin{array}{l}\text { Source } \\
\text { Ratio } \\
\end{array}$ & $\begin{array}{l}\text { Measured } \\
\text { Worth, } \$ \\
\end{array}$ & $\begin{array}{c}\text { Statistical } \\
\text { Uncertainty, \% }\end{array}$ & $\begin{array}{c}\text { Total } \\
\text { Uncertainty, } \%\end{array}$ \\
\hline A & 49 & 46 & 64 & 1.438 & 1.0153 & 2.224 & 0.111 & 0.873 \\
\hline B & 50 & 47 & 63 & 1.295 & 0.9994 & 1.985 & 0.0973 & 0.873 \\
\hline$B+b$ & 51 & 48 & 63 & 1.469 & 1.0128 & 3.982 & 0.108 & 0.866 \\
\hline$C$ & 52 & 49 & 64 & 1.418 & 0.9732 & 1.575 & 0.0958 & 0.876 \\
\hline$C+c$ & 53 & 50 & 64 & 1.445 & 0.9945 & 3.805 & 0.133 & 0.871 \\
\hline $\mathrm{D}$ & 54 & 51 & 64 & 1.190 & 0.9614 & 1.198 & 0.0968 & 0.882 \\
\hline$D+d$ & 55 & 52 & 63 & 1.054 & 0.9601 & 2.951 & 0.0999 & 0.869 \\
\hline$E$ & 56 & 53 & 63 & 1.133 & 0.9645 & 0.809 & 0.0809 & 0.894 \\
\hline$E+e$ & 57 & 54 & 63 & 1.470 & 0.9471 & 1.892 & 0.104 & 0.874 \\
\hline$F$ & 58 & 55 & 64 & 1.112 & 0.9761 & 0.532 & 0.101 & 0.919 \\
\hline$F+f$ & 59 & 56 & 64 & 1.470 & 0.9578 & 1.178 & 0.0930 & 0.885 \\
\hline$G$ & 60 & 57 & 64 & 0.865 & 0.9872 & 0.282 & 0.113 & 0.990 \\
\hline$G+g$ & 61 & 58 & 64 & 1.277 & 0.9744 & 0.591 & 0.108 & 0.916 \\
\hline CRP A & 91 & 88 & 64 & 1.436 & 1.0019 & 0.496 & 0.118 & 0.928 \\
\hline CRP F+f & 92 & 89 & 64 & 1.034 & 0.9877 & 0.359 & 0.139 & 0.957 \\
\hline
\end{tabular}


TABLE 2.4 Data Processing for ZPPR-18A 50\% Natural B C Control Rod Bank Measurements

\begin{tabular}{|c|c|c|c|c|c|c|c|c|c|}
\hline $\begin{array}{l}\text { Control } \\
\text { Rod } \\
\text { Pattern }\end{array}$ & $\begin{array}{l}\text { Drawer } \\
\text { Master }\end{array}$ & $\begin{array}{l}\text { Reactor } \\
\text { Loading } \\
\text { Number } \\
\end{array}$ & $\begin{array}{l}\text { Data } \\
\text { File } \\
\text { Number } \\
\end{array}$ & $\begin{array}{l}\text { Number } \\
\text { of FCs } \\
\end{array}$ & $x^{2}$ & $\begin{array}{l}\text { Source } \\
\text { Ratio } \\
\end{array}$ & $\begin{array}{l}\text { Measured } \\
\text { Worth, \$ }\end{array}$ & $\begin{array}{l}\text { Statistical } \\
\text { Uncertainty, } \&\end{array}$ & $\begin{array}{c}\text { Total } \\
\text { Uncertainty, } q \\
\end{array}$ \\
\hline $\begin{array}{c}6 \\
5 \\
12 \\
11 \\
21 \\
23 \\
24\end{array}$ & $\begin{array}{l}606 \\
606 \\
606 \\
606 \\
606 \\
606 \\
606\end{array}$ & $\begin{array}{l}63 \\
64 \\
65 \\
66 \\
67 \\
68 \\
69\end{array}$ & $\begin{array}{l}60 \\
61 \\
62 \\
63 \\
64 \\
65 \\
66\end{array}$ & $\begin{array}{l}64 \\
63 \\
63 \\
63 \\
64 \\
64 \\
63\end{array}$ & $\begin{array}{l}1.094 \\
1.299 \\
1.334 \\
1.169 \\
1.362 \\
1.306 \\
1.235\end{array}$ & $\begin{array}{l}1.0000 \\
1.0024 \\
0.9840 \\
0.9924 \\
0.9944 \\
0.9881 \\
0.9814\end{array}$ & $\begin{array}{l}1.286 \\
1.204 \\
0.842 \\
0.737 \\
0.407 \\
0.470 \\
0.514\end{array}$ & $\begin{array}{l}0.0937 \\
0.0880 \\
0.0875 \\
0.0927 \\
0.116 \\
0.113 \\
0.123\end{array}$ & $\begin{array}{l}0.881 \\
0.882 \\
0.894 \\
0.900 \\
0.945 \\
0.932 \\
0.925\end{array}$ \\
\hline $\begin{array}{c}14,18,20,24 \\
16,22\end{array}$ & $\begin{array}{l}605 \\
607\end{array}$ & 70 & 67 & 63 & 0.974 & 0.9533 & 3.050 & 0.0825 & 0.872 \\
\hline $\begin{array}{c}13,14,17,18,19,20,23,24 \\
15,16,21,22\end{array}$ & $\begin{array}{l}605 \\
604\end{array}$ & 71 & 68 & 61 & 1.475 & 0.9013 & 5.745 & 0.117 & 0.916 \\
\hline $\begin{array}{c}13,14,17,18,19,20,23 \\
15,16,21,22\end{array}$ & $\begin{array}{l}605 \\
607\end{array}$ & 72 & 69 & 61 & 1.287 & 0.9140 & 5.176 & 0.113 & 0.892 \\
\hline $\begin{array}{c}13,14,17,18,19,23 \\
15,16,21,22 \\
\text { and } 7-12\end{array}$ & $\begin{array}{l}605 \\
607 \\
606\end{array}$ & 73 & 70 & 57 & 1.359 & 0.8970 & 10.979 & 0.155 & 0.923 \\
\hline $\begin{array}{c}13,14,17,18,19,20,23,24 \\
15,16,21,22 \\
\text { and } 7-11\end{array}$ & $\begin{array}{l}605 \\
607 \\
606\end{array}$ & 74 & 71 & 59 & 1.464 & 0.8766 & 10.221 & 0.200 & 0.977 \\
\hline $\begin{array}{c}13,14,17,18,19,23,24 \\
15,16,21,22 \\
\text { and } 7-12\end{array}$ & $\begin{array}{l}605 \\
607 \\
606\end{array}$ & 75 & 72 & 59 & 1.361 & 0.8790 & 11.458 & 0.141 & 0.960 \\
\hline $\begin{array}{c}13,14,17,18,19,20,23,24 \\
15,16,21,22 \\
\text { and } 1-5\end{array}$ & $\begin{array}{l}605 \\
607 \\
606\end{array}$ & 76 & 73 & 58 & 1.514 & 0.9837 & 14.996 & 0.179 & 0.874 \\
\hline
\end{tabular}


TABLE 2.4 (contd)

\begin{tabular}{|c|c|c|c|c|c|c|c|c|c|}
\hline $\begin{array}{l}\text { Control } \\
\text { Rod } \\
\text { Pattern } \\
\end{array}$ & $\begin{array}{l}\text { Drawer } \\
\text { Master }\end{array}$ & $\begin{array}{l}\text { Reactor } \\
\text { Loading } \\
\text { Number }\end{array}$ & $\begin{array}{r}\text { Data } \\
\text { File } \\
\text { Number } \\
\end{array}$ & $\begin{array}{l}\text { Number } \\
\text { of FCs } \\
\end{array}$ & $x^{2}$ & $\begin{array}{l}\text { Source } \\
\text { Ratio }\end{array}$ & $\begin{array}{l}\text { Measured } \\
\text { Worth, } \$ \\
\end{array}$ & $\begin{array}{l}\text { Statistical } \\
\text { Uncertainty, } \$\end{array}$ & $\begin{array}{c}\text { Total } \\
\text { Uncertainty, } \$\end{array}$ \\
\hline $\begin{array}{c}13,14,17,18,19,20,23,24 \\
15,16,21,22 \\
\text { and } 1-6\end{array}$ & $\begin{array}{l}605 \\
607 \\
606\end{array}$ & 77 & 74 & 57 & 1.472 & 1.0048 & 17.026 & 0.188 & 0.875 \\
\hline $\begin{array}{c}13,14,17,18,19,20,23 \\
15,16,21,22 \\
\text { and } 1-6\end{array}$ & $\begin{array}{l}605 \\
607 \\
606\end{array}$ & 78 & 75 & 59 & 1.468 & 0.9919 & 15.547 & 0.151 & 0.868 \\
\hline $\begin{array}{l}1=6 \\
1-5\end{array}$ & $\begin{array}{l}606 \\
606\end{array}$ & $\begin{array}{l}79 \\
80\end{array}$ & $\begin{array}{l}76 \\
77\end{array}$ & $\begin{array}{l}61 \\
62\end{array}$ & $\begin{array}{l}1.179 \\
1.288\end{array}$ & $\begin{array}{l}1.0174 \\
1.0113\end{array}$ & $\begin{array}{l}7.119 \\
5.958\end{array}$ & $\begin{array}{l}0.135 \\
0.130\end{array}$ & $\begin{array}{l}0.867 \\
0.867\end{array}$ \\
\hline $\begin{array}{c}1-5 \\
\text { and } 7-12\end{array}$ & $\begin{array}{l}606 \\
605\end{array}$ & 81 & 78 & 59 & 1.486 & 1.0093 & 11.980 & 0.163 & 0.870 \\
\hline $\begin{array}{c}1-6 \\
\text { and } 7-11\end{array}$ & $\begin{array}{l}606 \\
605\end{array}$ & 82 & 79 & 61 & 1.494 & 0.9827 & 11.472 & 0.155 & 0.870 \\
\hline $\begin{array}{c}1-6 \\
\text { and } 7-12\end{array}$ & $\begin{array}{l}606 \\
605\end{array}$ & 83 & 80 & 59 & 1.523 & 1.0113 & 13.114 & 0.181 & 0.873 \\
\hline $\begin{array}{c}7-11 \\
7,-12 \\
13,24 \\
13,18,19,24 \\
15,16 \\
15,16,21,22\end{array}$ & $\begin{array}{l}606 \\
606 \\
606 \\
606 \\
606 \\
606\end{array}$ & $\begin{array}{l}84 \\
85 \\
86 \\
87 \\
88 \\
89\end{array}$ & $\begin{array}{l}81 \\
82 \\
83 \\
84 \\
85 \\
86\end{array}$ & $\begin{array}{l}63 \\
62 \\
63 \\
63 \\
63 \\
63\end{array}$ & $\begin{array}{l}1.122 \\
1.308 \\
1.274 \\
1.316 \\
1.216 \\
0.839\end{array}$ & $\begin{array}{l}0.9735 \\
0.9909 \\
0.9571 \\
0.9344 \\
0.9845 \\
0.9826\end{array}$ & $\begin{array}{l}4.772 \\
6.264 \\
0.910 \\
2.180 \\
0.705 \\
1.593\end{array}$ & $\begin{array}{l}0.100 \\
0.120 \\
0.127 \\
0.103 \\
0.108 \\
0.0834\end{array}$ & $\begin{array}{l}0.866 \\
0.866 \\
0.897 \\
0.878 \\
0.906 \\
0.877\end{array}$ \\
\hline
\end{tabular}


TABLE 2.5 Data Processing for Half Inserted 50\% B C Control Rods in ZPPR $18 \mathrm{~A}$

\begin{tabular}{|c|c|c|c|c|c|c|c|c|}
\hline $\begin{array}{l}\text { Control } \\
\text { Rods } \\
\end{array}$ & $\begin{array}{l}\text { Reactor } \\
\text { Loading } \\
\text { Number } \\
\end{array}$ & $\begin{array}{c}\text { Data } \\
\text { File } \\
\text { Number } \\
\end{array}$ & $\begin{array}{l}\text { Number } \\
\text { of FCs }\end{array}$ & $x^{2}$ & $\begin{array}{l}\text { Source } \\
\text { Ratio } \\
\end{array}$ & $\begin{array}{l}\text { Measured } \\
\text { Worth, } \$ \\
\end{array}$ & $\begin{array}{c}\text { Statistical } \\
\text { Uncertainty, \% }\end{array}$ & $\begin{array}{c}\text { Total } \\
\text { Uncertainty, }\end{array}$ \\
\hline $1-6$ & 94 & 91 & 59 & 1.354 & 1.0217 & 3.912 & 0.163 & 0.877 \\
\hline $\begin{array}{c}1-6 \text { and } \\
13-22,24\end{array}$ & 95 & 92 & 57 & 1.388 & 1.0023 & 7.874 & 0.188 & 0.877 \\
\hline $\begin{array}{c}1-6 \text { and } \\
13-23\end{array}$ & 96 & 93 & 57 & 1.508 & 1.0042 & 7.793 & 0.162 & 0.872 \\
\hline $\begin{array}{l}1-6 \text { and } \\
13-23 \text { and } \\
24 \text { Full In }\end{array}$ & 97 & 94 & 56 & 1.481 & 0.9981 & 8.577 & 0.155 & 0.870 \\
\hline $\begin{array}{c}1-6 \text { and } \\
13-24\end{array}$ & 98 & 95 & 54 & 1.501 & 1.0032 & 8.278 & 0.158 & 0.871 \\
\hline
\end{tabular}


TABLE 2.6 Worths of Control Rods on the $X$-axis

\begin{tabular}{|c|c|c|c|c|}
\hline $\begin{array}{l}\text { Data } \\
\text { File } \\
\end{array}$ & $\begin{array}{l}\text { Control } \\
\text { Rods } \\
\end{array}$ & $\begin{array}{l}\text { Measured } \\
\text { Worth, } \$ \\
\end{array}$ & $\begin{array}{l}\text { Calculated } \\
\text { Worth, } \$ \\
\end{array}$ & $\underline{C} / \mathrm{E}$ \\
\hline 46 & $A$ & 2.224 & 2.444 & 1.099 \\
\hline $\begin{array}{l}47 \\
48\end{array}$ & $\begin{array}{l}B \\
B+b\end{array}$ & $\begin{array}{l}1.985 \\
3.982\end{array}$ & $\begin{array}{l}2.183 \\
4.382\end{array}$ & $\begin{array}{l}1.100 \\
1.101\end{array}$ \\
\hline $\begin{array}{l}49 \\
50\end{array}$ & $\begin{array}{l}c \\
C+c\end{array}$ & $\begin{array}{l}1.575 \\
3.805\end{array}$ & $\begin{array}{l}1.742 \\
4.208\end{array}$ & $\begin{array}{l}1.106 \\
1.106\end{array}$ \\
\hline $\begin{array}{l}51 \\
52\end{array}$ & $\begin{array}{l}D \\
D+d\end{array}$ & $\begin{array}{l}1.198 \\
2.951\end{array}$ & $\begin{array}{l}1.317 \\
3.277\end{array}$ & $\begin{array}{l}1.099 \\
1.110\end{array}$ \\
\hline $\begin{array}{l}53 \\
54\end{array}$ & $\begin{array}{l}E \\
E+e\end{array}$ & $\begin{array}{l}0.809 \\
1.892\end{array}$ & $\begin{array}{l}0.896 \\
2.116\end{array}$ & $\begin{array}{l}1.108 \\
1.118\end{array}$ \\
\hline $\begin{array}{l}55 \\
56\end{array}$ & $\begin{array}{l}F \\
F+f\end{array}$ & $\begin{array}{l}0.532 \\
1.178\end{array}$ & $\begin{array}{l}0.585 \\
1.301\end{array}$ & $\begin{array}{l}1.100 \\
1.104\end{array}$ \\
\hline $\begin{array}{l}57 \\
58\end{array}$ & $\begin{array}{l}G \\
G+g\end{array}$ & $\begin{array}{l}0.282 \\
0.591\end{array}$ & $\begin{array}{l}0.309 \\
0.654\end{array}$ & $\begin{array}{l}1.096 \\
1.107\end{array}$ \\
\hline $\begin{array}{l}88 \\
89\end{array}$ & $\begin{array}{l}\text { CRP A } \\
\text { CRP } F+f\end{array}$ & $\begin{array}{l}0.496 \\
0.359\end{array}$ & $\begin{array}{l}0.606 \\
0.425\end{array}$ & $\begin{array}{l}1.222 \\
1.184\end{array}$ \\
\hline \multicolumn{5}{|c|}{ Relative to CRPs } \\
\hline $\begin{array}{ll}46, & 48 \\
56, & 89\end{array}$ & $\begin{array}{l}A \\
F+f\end{array}$ & $\begin{array}{l}1.728 \\
0.819\end{array}$ & $\begin{array}{l}1.838 \\
0.876\end{array}$ & $\begin{array}{l}1.064 \\
1.070\end{array}$ \\
\hline
\end{tabular}


TABLE 2.7 Interaction Effects for Control Rods Along the X-Axis

\begin{tabular}{|c|c|c|c|c|}
\hline $\begin{array}{c}\text { Control } \\
\text { Rods } \\
\end{array}$ & $\begin{array}{c}\text { Separation } \\
\mathrm{cm}\end{array}$ & $\begin{array}{c}\text { Interaction } \\
\% \\
\end{array}$ & $\begin{array}{c}\text { Normalized } \\
\text { Interaction } \\
\% \$^{-1 a} \\
\end{array}$ & $\begin{array}{c}\text { Calculated } \\
\text { Interaction } \\
q \\
\end{array}$ \\
\hline$B, b$ & 66.3 & 0.30 & 0.15 & 0.37 \\
\hline$C, c$ & 132.6 & 20.8 & 13.2 & 20.8 \\
\hline$D, d$ & 187.8 & 23.2 & 19.3 & 24.4 \\
\hline$E, e$ & 243.1 & 16.9 & 20.9 & 18.1 \\
\hline$F, f$ & 270.7 & 10.7 & 20.1 & 11.2 \\
\hline$G, g$ & 303.8 & 4.8 & 17.0 & 5.8 \\
\hline
\end{tabular}

Interaction $\%$ divided by the worth of a single rod. 
TABLE 2.8 Worths of Control Rods in the Three Banks

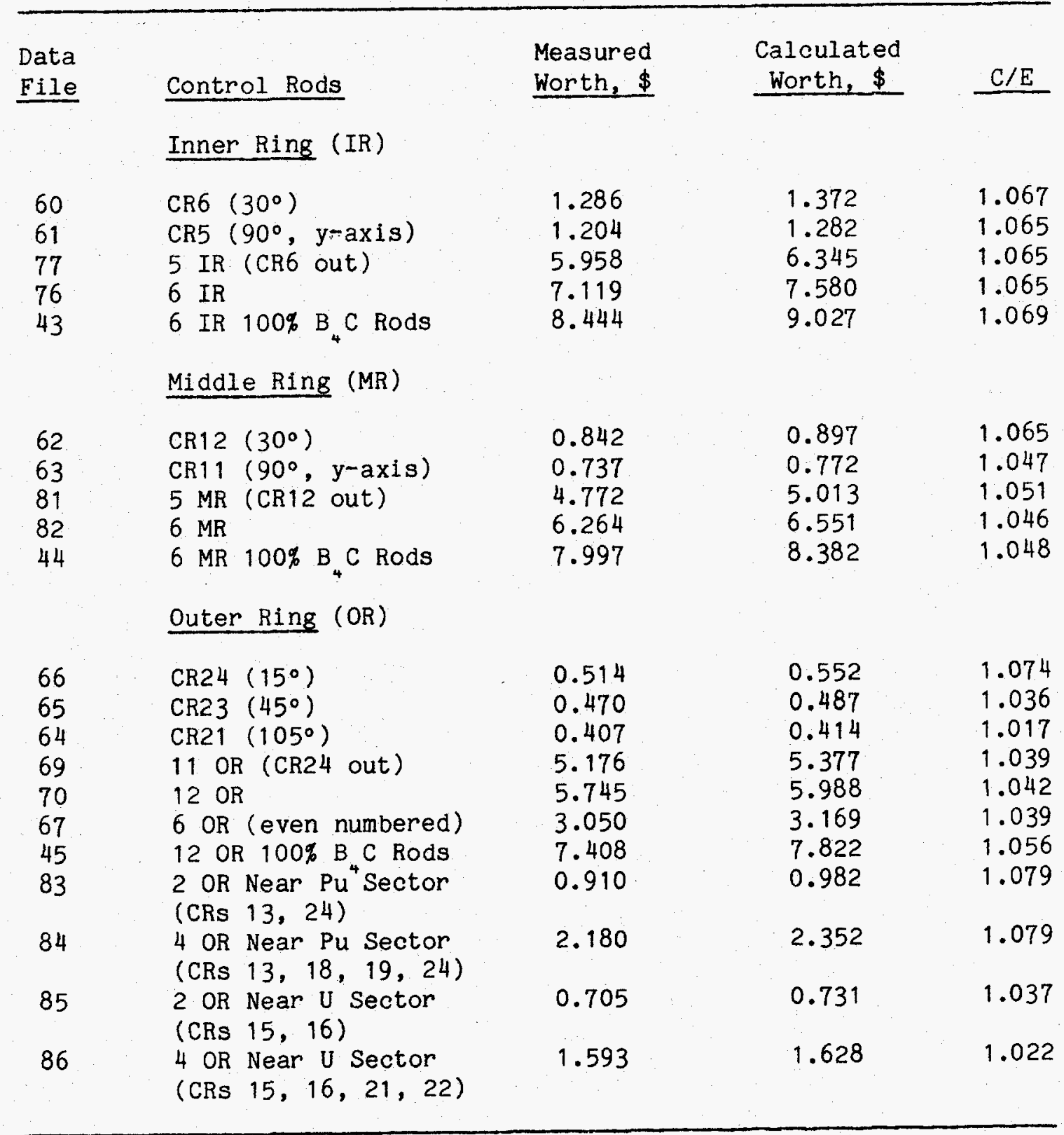


TABLE 2.9 Worths of Rod Bank Combinations and Interaction Effects

\begin{tabular}{|c|c|c|c|c|}
\hline File & Control Rods & $\begin{array}{l}\text { Measured } \\
\text { Worth, \$ } \\
\end{array}$ & $\begin{array}{l}\text { Calculated } \\
\text { Worth, } \$ \\
\end{array}$ & $C / E$ \\
\hline $\begin{array}{l}80 \\
76 \\
82\end{array}$ & $\begin{array}{l}6 \mathrm{IR}+6 \mathrm{MR} \\
6 \mathrm{IR} \\
6 \mathrm{MR} \\
\text { Sum } 6 \mathrm{IR}, 6 \mathrm{MR} \\
\text { Interaction }\end{array}$ & $\begin{array}{r}13.114 \\
7.119 \\
6.264 \\
13.383 \\
-2.0 \%\end{array}$ & $\begin{array}{r}13.814 \\
7.580 \\
6.551 \\
14.131 \\
-2.2 \%\end{array}$ & $\begin{array}{l}1.053 \\
1.065 \\
1.046 \\
1.056\end{array}$ \\
\hline $\begin{array}{l}74 \\
76 \\
70\end{array}$ & $\begin{array}{l}6 \mathrm{IR}+12 \mathrm{OR} \\
6 \mathrm{IR} \\
12 \mathrm{OR} \\
\text { Sum } 6 \mathrm{IR}, 12 \mathrm{OR} \\
\text { Interaction }\end{array}$ & $\begin{array}{r}17.026 \\
7.119 \\
5.745 \\
12.864 \\
+32 \%\end{array}$ & $\begin{array}{r}17.574 \\
7.580 \\
5.988 \\
13.568 \\
+30 \%\end{array}$ & $\begin{array}{l}1.032 \\
1.065 \\
1.042 \\
1.055\end{array}$ \\
\hline $\begin{array}{l}72 \\
82 \\
70\end{array}$ & $\begin{array}{l}6 \mathrm{MR}+12 \mathrm{OR} \\
6 \mathrm{MR} \\
12 \mathrm{OR} \\
\text { Sum } 6 \mathrm{MR}, 12 \mathrm{OR} \\
\text { Interaction }\end{array}$ & $\begin{array}{l}11.458 \\
6.264 \\
5.745 \\
12.009 \\
-4.6 \%\end{array}$ & $\begin{array}{c}12.030 \\
6.551 \\
5.988 \\
12.539 \\
-4.1 \%\end{array}$ & $\begin{array}{l}1.050 \\
1.046 \\
1.042 \\
1.044\end{array}$ \\
\hline
\end{tabular}


TABLE 2.10 Worths of Rod Bank Combinations with Missing Rods

\begin{tabular}{|c|c|c|c|c|}
\hline File & Control Rods & $\begin{array}{l}\text { Measured } \\
\text { Worth, } \$ \\
\end{array}$ & $\begin{array}{l}\text { Calculated } \\
\text { Worth, } \$ \\
\end{array}$ & $C / E$ \\
\hline 78 & $5 \mathrm{IR}+6 \mathrm{MR}$ & 11.980 & 12.587 & 1.051 \\
\hline 79 & $6 \mathrm{IR}+5 \mathrm{MR}$ & 11.472 & 12.100 & 1.055 \\
\hline 80 & $6 \mathrm{IR}+6 \mathrm{MR}$ & 13.114 & 13.814 & 1.053 \\
\hline 73 & $5 \mathrm{IR}+12 \mathrm{OR}$ & 14.996 & 15.501 & 1.034 \\
\hline 75 & $6 I R+11 O R$ & 15.547 & 16.095 & 1.035 \\
\hline 74 & $6 I R+12 O R$ & 17.026 & 17.574 & 1.032 \\
\hline 71 & $5 \mathrm{MR}+12 \mathrm{OR}$ & 10.221 & 10.742 & 1.051 \\
\hline 70 & $6 \mathrm{MR}+11 \mathrm{OR}$ & 10.979 & 11.460 & 1.044 \\
\hline 72 & $6 \mathrm{MR}+12 \mathrm{OR}$ & 11.458 & 12.030 & 1.050 \\
\hline
\end{tabular}


TABLE 2.11 Variations in the Worth of a Single Control Rod

\begin{tabular}{|c|c|c|c|c|c|}
\hline $\begin{array}{c}\text { Control } \\
\text { Rod } \\
\end{array}$ & $\begin{array}{l}\text { Rods in } \\
\text { Core }\end{array}$ & $\begin{array}{l}\text { Measured } \\
\text { Worth, } \$ \\
\end{array}$ & $\begin{array}{c}\text { Statistical } \\
\text { Uncertainty, } \% \\
\end{array}$ & $\begin{array}{l}\text { Calculated } \\
\text { Worth, } \$ \\
\end{array}$ & $C / E$ \\
\hline $\begin{array}{l}\text { CR6 } \\
\text { (Inner Ring) }\end{array}$ & $\begin{array}{l}\text { None } \\
5 \mathrm{IR} \\
5 \mathrm{IR}+6 \mathrm{MR} \\
5 \mathrm{IR}+12 \mathrm{OR}\end{array}$ & $\begin{array}{l}1.286 \\
1.161 \\
1.134 \\
2.030\end{array}$ & $\begin{array}{l}0.1 \\
1.1 \\
2.7 \\
2.1\end{array}$ & $\begin{array}{l}1.372 \\
1.235 \\
1.227 \\
2.073\end{array}$ & $\begin{array}{l}1.067 \\
1.064 \\
1.082 \\
1.021\end{array}$ \\
\hline $\begin{array}{l}\text { CR12 } \\
\text { (Middle Ring) }\end{array}$ & $\begin{array}{l}\text { None } \\
5 \mathrm{MR} \\
6 \mathrm{IR}+5 \mathrm{MR} \\
5 \mathrm{MR}+12 \mathrm{OR}\end{array}$ & $\begin{array}{l}0.842 \\
1.492 \\
1.642 \\
1.237\end{array}$ & $\begin{array}{l}0.1 \\
0.6 \\
1.8 \\
2.1\end{array}$ & $\begin{array}{l}0.897 \\
1.538 \\
1.714 \\
1.288\end{array}$ & $\begin{array}{l}1.065 \\
1.031 \\
1.044 \\
1.041\end{array}$ \\
\hline $\begin{array}{l}\text { CR2 } 4 \\
\text { (Outer Ring) }\end{array}$ & $\begin{array}{l}\text { None } \\
11 \mathrm{OR} \\
6 \mathrm{IR}+11 \mathrm{OR} \\
6 \mathrm{MR}+11 \mathrm{OR}\end{array}$ & $\begin{array}{l}0.514 \\
0.569 \\
1.479 \\
0.479\end{array}$ & $\begin{array}{l}0.1 \\
1.6 \\
2.7 \\
4.9\end{array}$ & $\begin{array}{l}0.552 \\
0.611 \\
1.479 \\
0.570\end{array}$ & $\begin{array}{l}1.074 \\
1.074 \\
1.000 \\
1.190\end{array}$ \\
\hline
\end{tabular}


TABLE 2.12 Worths of Half-Inserted Control Rods

\begin{tabular}{|c|c|c|c|c|}
\hline $\begin{array}{l}\text { Data } \\
\text { File }\end{array}$ & Control Rods & $\begin{array}{l}\text { Measured } \\
\text { Worth, } \$\end{array}$ & $\begin{array}{l}\text { Calculated } \\
\text { Worth, } \$\end{array}$ & $C / E$ \\
\hline 91 & 6 IR & 3.912 & 4.395 & 1.123 \\
\hline 95 & $6 \mathrm{IR}+12 \mathrm{OR}$ & 8.278 & 9.304 & 1.124 \\
\hline 92 & $\begin{array}{l}6 \text { IR }+11 \text { oR } \\
\text { (CR23 out) }\end{array}$ & 7.874 & 8.834 & 1.122 \\
\hline 93 & $\begin{array}{l}6 \text { IR }+11 \text { OR } \\
\text { (CR24 out) }\end{array}$ & 7.793 & 8.748 & 1.123 \\
\hline 94 & $\begin{array}{l}6 \text { IR }+11 \text { OR } \\
+ \text { CR24 full in }\end{array}$ & 8.577 & 9.595 & 1.119 \\
\hline
\end{tabular}


TABLE 2.13 Three-dimensional Calculations of Rod Worths in $\mathrm{ZPPR}=18 \mathrm{~A}$

\begin{tabular}{|c|c|c|c|c|c|}
\hline \multirow{2}{*}{$\begin{array}{l}\text { Control Rods } \\
\text { Subcritical Reference }\end{array}$} & \multicolumn{2}{|c|}{ Nodal Diffusion } & \multicolumn{2}{|c|}{ Nodal Transport } & \multirow[t]{2}{*}{$\begin{array}{c}\text { Transport } \\
\text { Correction, } \%\end{array}$} \\
\hline & 0.989782 & - & 0.991872 & - & \\
\hline $\begin{array}{l}6 \text { IR } 100 \% \text { B C } \\
6 \text { MR } 100 \% B^{4} \mathrm{C} \\
12 \text { OR } 100 \%{ }^{4}{ }_{4} C\end{array}$ & $\begin{array}{l}0.956750 \\
0.958681 \\
0.961627\end{array}$ & $\begin{array}{l}9.213 \\
8.657 \\
7.813\end{array}$ & $\begin{array}{l}0.959626 \\
0.961517 \\
0.964024\end{array}$ & $\begin{array}{l}8.948 \\
8.407 \\
7.693\end{array}$ & $\begin{array}{l}-2.9 \\
-2.9 \\
-1.5\end{array}$ \\
\hline $\begin{array}{l}6 \mathrm{IR} \\
6 \mathrm{MR} \\
12 \mathrm{OR}\end{array}$ & $\begin{array}{l}0.962207 \\
0.965714 \\
0.968157\end{array}$ & $\begin{array}{l}7.648 \\
6.651 \\
5.961\end{array}$ & $\begin{array}{l}0.964952 \\
0.968345 \\
0.970465\end{array}$ & $\begin{array}{l}7.429 \\
6.470 \\
5.874\end{array}$ & $\begin{array}{l}-2.9 \\
-2.7 \\
-1.5\end{array}$ \\
\hline $\begin{array}{l}4 \text { OR Pu Sector } \\
4 \text { OR U Sector }\end{array}$ & $\begin{array}{l}0.981229 \\
0.983795\end{array}$ & $\begin{array}{l}2.326 \\
1.624\end{array}$ & $\begin{array}{l}0.983354 \\
0.986010\end{array}$ & $\begin{array}{l}2.307 \\
1.583\end{array}$ & $\begin{array}{l}-0.8 \\
-2.5\end{array}$ \\
\hline
\end{tabular}


TABLE 2.14 Diffusion and Transport Results for Rod Worths in ZPPR $-18 \mathrm{~A}$

\begin{tabular}{lccc}
\hline Control Rods & $\begin{array}{c}\text { Measured } \\
\text { Worth, } \$\end{array}$ & $\begin{array}{c}\text { Nodal } \\
\text { Diffusion } \\
\mathrm{C} / \mathrm{E}\end{array}$ & $\begin{array}{c}\text { Nodal } \\
\text { Transport } \\
\mathrm{C} / \mathrm{E}\end{array}$ \\
\hline 6 IR 100\% B C & 8.444 & 1.091 & 1.060 \\
$6 \mathrm{MR} \mathrm{100 \%} \mathrm{B} \mathrm{C}$ & 7.997 & 1.083 & 1.051 \\
12 OR 100\% B C & 7.408 & 1.055 & 1.038 \\
6 IR & & & \\
6 MR & 7.119 & 1.074 & 1.044 \\
12 OR & 6.264 & 1.062 & 1.033 \\
& 5.745 & 1.038 & 1.022 \\
4 OR Pu Sector & & & 1.058 \\
4 OR U Sector & 2.180 & 1.067 & 0.994 \\
\hline
\end{tabular}




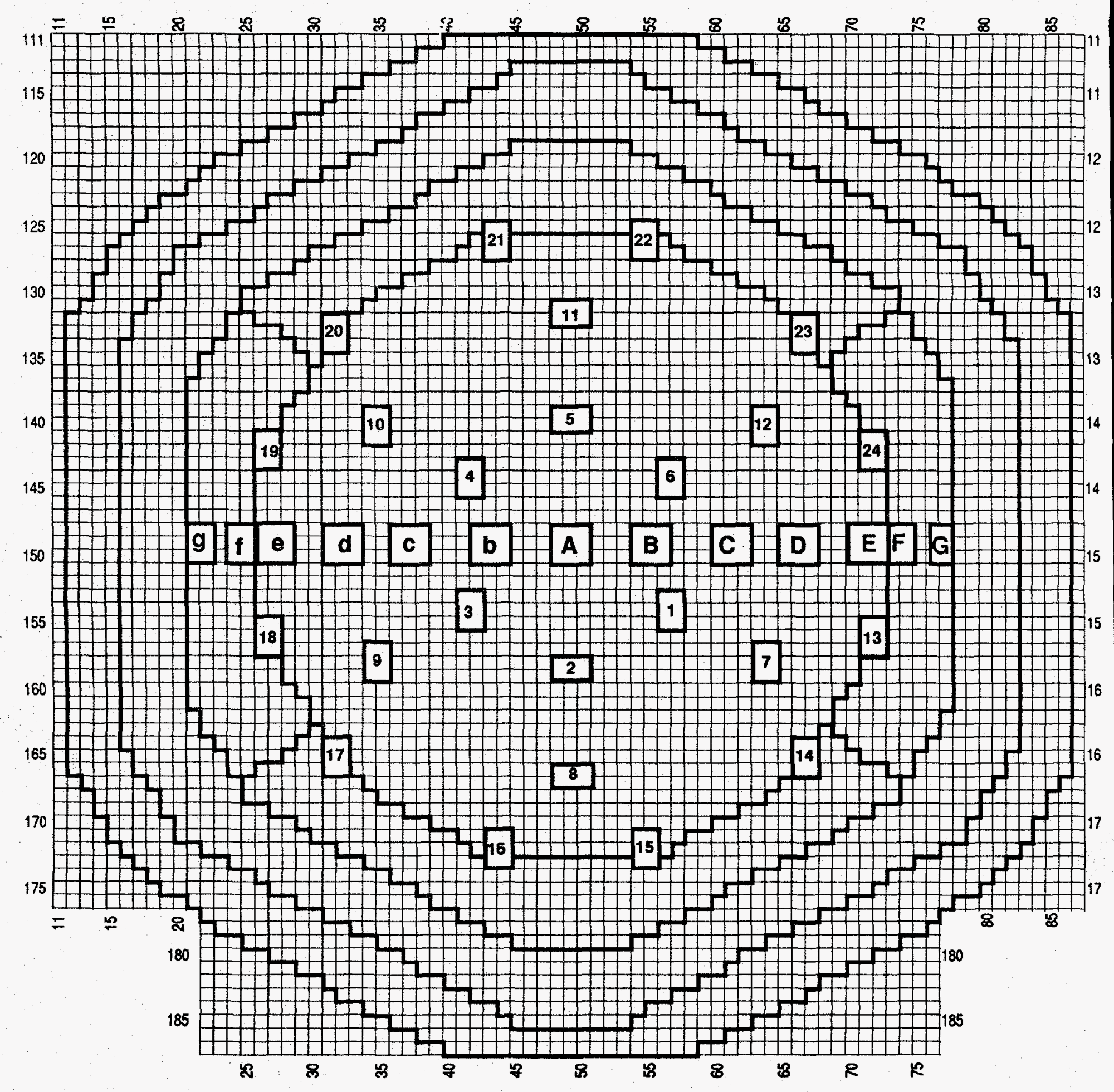

Figure $2.1^{\circ}$ Control Rod Locations in ZPPR-18 


\section{THE CONTROL ROD WORTH MEASUREMENT IN ZPPR-18B \\ (F. Nakashima* and P.J. Collins)}

ZPPR-18B was critical with the inner bank of six control rods and the outer bank of twelve rods half inserted. The raison d'être of 18B was to measure reaction rate distributions (Section 4) but the opportunity was taken to measure the worth of inserting the middle bank of control rods. This measurement provides a test of calculating the shutdown reactivity of the secondary control bank with the primary rods near the beginning-of-cycle position.

The reference for the measurement was the ZPPR -18B subcritical loading described in ANL-ZPR-485, p. 15. The control rod measurement was made on December 4, 1987 in reactor run 95, loading 106. Fission chamber countrates and other operational data were recorded on ZPPR-18 64 detector file 104. The reactivity of the subcritical reference was $-32.5 \phi(1 \sigma=0.28 \phi)$ and countrate data were recorded on file 102.

The middle bank control rods were constructed with drawer master 606 and compositions are given in Section 2, Table 1 of this report.

The calculation of detector efficiencies and source ratio used a 6 group $x y$ diffusion model for $Z P P R-18 B$. The control positions with half inserted rods in the reference core used a mixture of control rod and CRP compositions and bucklings, with the buckling terms taken from the ZPPR $18 \mathrm{~A}$ control rod calculation. The calculation for the reference core was adjusted to 0.990600 (close to the k-effective for ZPPR-18A with this model) by using a mixture of $34 \%$ control rod and $66 \% \mathrm{CRP}$ in the inner and outer bank positions. Then, inserting the middle ring rods (at full denstty) gave a k-effective of 0.966521 and a calculated worth of $6.604 \$$ using a B-effective of $0.3801 \%$.

\footnotetext{
*0n assignment from the Power Reactor and Nuclear Fuel Development Corporation.
} 
The data processing for this measurement is shown in Table 3.1. Results for all four methods used in the McCRUNCH code are compared: (i) using all 64 fission chambers, (ii) rejecting detectors which predict reactivities greater than 3.6 standard deviation from the mean, (iii) least squares fit of reactivity versus efficiercy, and (iv) least squares fit with adjustment to the calculated source ratio. The fit to the experimental countrates is excellent with no more than four detectors being rejected. This gives confidence in the simple calculation model used and in the source ratio. The preferred reactivity worth is taken from the last case, 1.e., 6.181\$ which has an acceptable value of chi-square and yields a total uncertainty of $0.92 \%$.

The ratio of calculation to experiment using the $x y$ diffusion model is 1.068 which compares with 1.046 for the worth of the middle bank in ZPPR-18A. 
TABLE 3.1 Data Processing for the Measurement of the Worth of the Middle Bank of Control Rods in ZPPR-18B

\begin{tabular}{|c|c|c|c|c|}
\hline & $\begin{array}{c}\text { Mean } \\
\text { using all } \\
\text { Detectors } \\
\end{array}$ & $\begin{array}{c}\text { Mean with } \\
\text { Rejection } \\
>3.60 \\
\end{array}$ & $\begin{array}{l}\text { Least } \\
\text { Squares } \\
\text { Fit (LSFIT) }\end{array}$ & $\begin{array}{c}\text { LSFIT with } \\
\text { Adjusted } \\
\text { Source Ratio } \\
\end{array}$ \\
\hline Number of Detectors & 64 & 60 & 63 & 63 \\
\hline Reduced chi-square & 5.95 & 1.78 & 1.28 & 1.28 \\
\hline Source Ratio & 0.9977 & 0.9977 & 0.9977 & 0.9974 \\
\hline Worth, $\$$ & 6.175 & 6.157 & 6.183 & 6.181 \\
\hline Statistical Uncertainty, $1 \sigma, \%$ & 0.20 & 0.17 & 0.10 & 0.10 \\
\hline Correlated Uncertainty, $1 \sigma, \%$ & 0.92 & 0.92 & 0.92 & 0.92 \\
\hline Total Uncertainty $10, \%$ & 0.94 & 0.93 & 0.92 & 0.92 \\
\hline
\end{tabular}


4. MEASUREMENTS AND ANALYSIS OF REACTION RATES IN ZPPR-18B (P. J. COIIInS, J. M. Gasidlo, D. W. Maddison, and G. L. Grassesch1)

\subsection{Introduction}

ZPPR $-18 \mathrm{~B}$ was a simulation of a large conventional LMR design with the primary rod banks - 6 inner ring and 12 outer ring - half inserted. Reaction rate distributions were measured in detail in or der to compare the accuracy of predictions with those for $Z P P R-18 \mathrm{~A}$ in which no control rods were inserted.

The reactor zones in ZPPR -18B were identical to those in ZPPR $18 \mathrm{~A}$. The reactivity of the inserted control rods was compensated by increasing enrichments in the inner and outer core regions. Enrichment changes were approximately uniform within each zone but an additional inorease in enrichment was made in the uranium-fuel zones. in the outer core in order to improve the power distributions. A detalled description of $Z P P R-18 B$ is given in $A N L-Z P R-485$ p. 15.

This report contains the experimental "cell-averaged" reaction rate data, together with the analysis using ENDF/B-V.2 cross sections and an xyz nodal transport calculation consistent with that used for ZPPR-18A. The basic foil data and the cell-average/foll adjustment factors will be given in a subsequent section.

\subsection{Description of the Measurements}

Measurements in ZPPR-18B were made in both halves of the core in order to follow the effects of the half-inserted control rods on the fission rates. Two irradiations were necessary to handle the large number of foils. In addition, the in-cell measurements, using multiple foils in selected cells, were made in $2 P P R-18 B$ to provide data for deriving the experimental celleaverage/foll factors.

The first irradiation was made in loading 112 , reactor run 102 , on December 10, 1987. Eight ZPPR operating control rods, in matrix 135-67 and 
symmetric positions, were inserted to a depth of $42.8 \mathrm{~cm}$ from the midplane and had an average worth of $6.08 \notin$ during the irradiation. The second irradiation was made in loading 115, Run 105 on December 15, 1987 and the shim rods were inserted $43.2 \mathrm{~cm}$ from the midplane with a worth of $5.83 \phi$.

A set of ten ${ }^{235} \mathrm{U}$ foils were irradiated in the same locations in each irradiation. The average ratio of the corresponding countrates was 1.0008 with a standard deviation of the distribution of 0.0020 . The detailed comparison will be given with the basic data report. This agreement enabled the data from the two irradiations to be combined into one set without further adjustment.

Statistical uncertainties in the measured reaction rates were essentially the same as for ZPPR-18A - around $0.7 \%$ for the three nonthreshold reactions in the core regions and $1.5 \%$ to $2.5 \%$ for fission in ${ }^{238} \mathrm{U}$. Foil techniques are described in $\mathrm{ANL}-82-38$ and calibrations were consistent with these used throughout $Z P P R-17$ and $Z P P R-18$ as described in ANL-87-5.

Reaction rate distributions were measured in the same positions as in $Z P P R-18 \mathrm{~A}$, but extended to both halves of the reactor:

(i) Radial distributions along the $x$-axis at $+5 \mathrm{~cm},-5 \mathrm{~cm}$, $+28 \mathrm{~cm}$, and $-28 \mathrm{~cm}$ from the midplane.

(ii) A radial distribution along the y-axis for ${ }^{235} U$ fission only.

(iii) Radial distributions at $15^{\circ}$ to the $x$-axis, passing through an outer ring CRP (half-1) and outer ring control rod (half-2) at $\pm 5 \mathrm{~cm}$ and $\pm 28 \mathrm{~cm}$.

(iv) Radial distributions at $30^{\circ}$ to the $x$-axis, passing through an inner-ring control rod and a middle-ring CRP. These measurements were made in half -2 only at $Z=-5 \mathrm{~cm}$ and $-28 \mathrm{~cm}$. 
(v) Axial distributions at the core center (matrix 1,249-49), in the inner core (matrix 1,249-64), in the outer core (matrix $1,249-75)$ and adjacent to an inner bank CRP/control rod (matrix 1,246-57).

A total of 895 reaction rate values were measured. The number of plutonium foils was limited. As for ZPPR-18A, these were used for full radial traverses along the $x$-axis, at $15^{\circ}$ to the xraxis and in an axial traverse at the core center. However, in ZPPR-18B the plutonium foils were used only in half-2 for radial distributions (control rod half) and were made in both halves for the axial distribution at the core center. In general, ${ }^{235} \mathrm{U}$ fission was measured in corresponding locations in both halves but, apart from the axial distributions, ${ }^{23 \mathrm{U}} \mathrm{U}$ capture and fission were measured only in half-2. Thus, the detailed mapping of the core fission distributions is available only for $235 \mathrm{U}$ fission.

The foll locations in the $x y$ plane are shown in ANL-ZPR-489, p. 57 for ZPPR-18A. The foil types shown in the figure correspond to those in half -2 of $\mathrm{ZPPR}-18 \mathrm{~B}$ as noted above.

\subsection{Calculation Methods}

Calculations were made with the cross sections processed for ZPPR$18 \mathrm{~A}$ using the "buckling-recycle" method (ANLrZPR-489, p. 12). The data were not reprocessed for ZPPR-18B. The calculations used 21 group cross sections in an xyz model with the nodal transport path of DIF3D and included anisom tropic transport cross sections. The model required a fulliz representation and, because of storage limitations on the IBM computer at the time, it was necessary to use the cubic polynomial approximation rather than the quartic polynomials which are normally used. (A subsequent run using the CRAY com puter gave an increase in kreffective of $0.035 \%$ using the quartic approximation rather than the cubic). The calculation model is given in ANL-ZPR-489, p. 26 .

The shim rods were inserted in the model to a depth of $43.3 \mathrm{~cm}$ from the midplane and used special boron carbide cross sections appropriate 
to homogenizing the shim blades over the matrix area. The k-effectives were 0.994140 with shim rods inserted and 0.994524 with shim rods withdrawn (cubic polynomial approximation), giving a reactivity of $10.2 \varnothing$. This compares rather poorly with the measured worth of $6 \phi$ (Section 2). The error is supposed to be due to the experimental control rods in half-2 being immediately adjacent to the shim rods so that the data homogenized over the matrix area is a poor represertation of the actual shim blade location. However, since the experimental rods have a much larger worth than the partly inserted shim blades (about $1 \$$ for 4 half-inserted outer ring rods) it is assumed that mispredictions of the shim rod worth have a negligible effect on the calculated renction rate distributions.

Calculated reaction rates were interpolated to the foll positions of the measurements using a quadratic $f$ it to the node-average values.

\subsection{Experimental Reaction Rates and Comparison with Calculation}

The reaction rate results are given in Tables 4.1 to 4.14 . The data are organized into distributions along the axes, at $15^{\circ}$ and $30^{\circ}$ to the $x$ raxis and into axial distributions. As for ZPPR $=18 \mathrm{~A}$ (ANL-ZPR $489, \mathrm{p} .50$ et seq.), reactor zones are indicated by $I C, O C, R B, A B$ in an obvious notar tion and the single column drawer types are distinguished by SF (drawers with $\mathrm{Fe}_{2} \mathrm{O}_{3}$ adjacent to fuel) and by $S M$ (depleted uranium metal adjacent to fuel).

Experimental reaction rates are given in units of $10^{-1} \mathrm{~B}$ reactions per atom per second at a reactor power of approximately 1 watt. In the analysis, calculations are normalized to give a mean $\mathrm{C} / \mathrm{E}$ of unity for the 78 measurements of fission in ${ }^{23 y} \mathrm{Pu}$ which were made along the $\mathrm{x}$-axis and at $15^{\circ}$ to the axis at $z=-5 \mathrm{~cm}$ and for an axial distribution at the core center.

Predictions of reaction rate distributions are similar to those in ZPPR-18A, except for positions in the vicinity of inserted control rods:

(i) Radial distributions along the $x$-axis and at $15^{\circ}$ and $30^{\circ}$ are well predicted. In the inner core, the $C / E$ values are 
constant within the uncertainties. The nonthreshold reaction rates in the outer core have, on average, a lower $C / E$ by $0.5 \%$ to $1 \%$ than in the inner core. Measured results for ${ }^{235} \mathrm{U}$ fission at $15^{\circ}$ to the $\mathrm{x}^{-}$axis and at four axial heights are shown in Fig. 4.1 .

(ii) Fission rates along the yraxis show a decreasing $\mathrm{C} / \mathrm{E}$ with radius and are predicted $5 \%$ low in the outer core uranium sector relative to the core center (Table 4.4).

(iii) Fission rates in ${ }^{23} \mathrm{Pu}$ and ${ }^{235} \mathrm{U}$ have $\mathrm{C} / \mathrm{E}$ values $1 \%$ higher in the SM drawers than in the SF drawers.

(iv) Reaction rates in half-two of the assembly (negative $z$ in the tables) intersect an outer ring control rod in the $15^{\circ}$ distributions (Tables 4.5, 4.6 and 4.7) and intersect an inner ring control rod in the $30^{\circ}$ distributions (Table 4.8). The $\mathrm{C} / \mathrm{E}$ values for ${ }^{23 y} \mathrm{Pu}$ and ${ }^{235} \mathrm{U}$ are $1-3 \%$ higher in drawers near to the rods than elsewhere.

(v) Axial distributions are well predicted in the core regions with standard deviations of the C/E distributions close to the statistical uncertainties (Tables 4.9 to 4.14 ). In the axial blankets, the $\mathrm{C} /$ Es increase with penetration indicating errors in predicting effects of the steel axial reflector. Calculated and measured results for the axial ${ }^{235} \mathrm{U}$ fission distribution near the core center are shown in Fig. 4.2.

(vi) Axial fission rates adjacent to a half-inserted control rod in the inner ring (matrix 246,146-57) are given in Table 4.14 and illustrated in Fig. 4.3. The average C/E results in the core are in good agreement -1.000 (S.D. $=0.007$ ) adjacent to the control rod and 0.995 (S.D. $=0.011$ ) adjacent to the CRP. The predictions in the axial blanket are a few percent different to those in positions removed from the control rod position. 
The radial reaction rate analysis for ${ }^{235} \mathrm{U}$ fission is summarized in Figs. 4.4 to 4.7 . These figures show the mean $C / E$ results for groups of 3 to 4 adjacent locations at $5 \mathrm{~cm}$ and $28 \mathrm{~cm}$ above and below the midplane.

Table 4.15 gives the mean C/E results for the inner and outer core at the same axial heights. Results for the distributions at the $x$-axis, $15^{\circ}$ and $30^{\circ}$, have been combined in the table. In the all-CRP half $(z=+5 \mathrm{~cm}$ and $+28 \mathrm{~cm})$, the standard deviations are rather larger than statistics. The mean $C / E$ results are $0.5 \%$ to $1 \%$ higher in the control rod half. This table also shows slightly lower $C / E$ values in the outer core relative to the inner core.

\subsection{Reaction Rate Ratios}

Reaction rate ratios relative to ${ }^{239} \mathrm{Pu}$ fission are given in Tables 4.16 to 4.18. The experimental values shown are simply the ratios at the respective foil locations and have not been interpolated to a common location.

A summary of the analysis is given in Table 4.19. Results for the ${ }^{235} \mathrm{U}$ fission ratio (F5/F9) and the ${ }^{230} \mathrm{U}$ capture ratio (C8/F9) are consistent among the three distributions. Results for the ${ }^{238} \mathrm{U}$ fission ratio are less consistent between the inner core and the outer core. The mean $C / E$ values compare well with those obtained from analysis of ZPPR-18A as shown below: $\dagger$

$\begin{array}{llll} & & \text { Mean } & \text { Standard Deviation } \\ \text { F5/F9 } & \text { ZPPR-18B } & 1.003 & 0.009 \\ & \text { ZPPR-18A } & 1.004 & 0.010 \\ \text { C8/F9 } & \text { ZPPR-18B } & 1.042 & 0.012 \\ & \text { ZPPR-18A } & 1.046 & 0.010 \\ \text { F8/F9 } & \text { ZPPR-18B } & 1.002 & 0.024 \\ & \text { ZPPR-18A } & 0.998 & 0.023\end{array}$

The measured results for $238 \mathrm{U}$ fission in $Z P R-489$ have been revised. The corrected values will be published in ANL-ZPR- 492 . 
TABLE 4.1 ZPPR 18B: Radial Reaction Rate Distributions along the $\mathrm{x}$-axis at $\mathrm{z}=-5.16 \mathrm{~cm}$

\begin{tabular}{|c|c|c|c|c|c|c|c|}
\hline \multirow[b]{2}{*}{ Matrix } & \multirow[b]{2}{*}{ Zone } & \multicolumn{2}{|c|}{$2{ }^{3 y} \mathrm{Pu}(\mathrm{n}, \mathrm{f})$} & \multicolumn{2}{|c|}{${ }^{2{ }^{38}} \mathrm{U}(\mathrm{n}, \gamma)$} & \multicolumn{2}{|c|}{$238 U(n, f)$} \\
\hline & & Exp. & $C / E$ & Exp. & $C / E$ & Exp. & $C / E$ \\
\hline 249.49 & IC $\mathrm{SF}$ & 8.283 & 0.998 & 1.1620 & 1.040 & 0.1945 & 1.029 \\
\hline 24950 & IC SE & 8.295 & 0.995 & 1.1680 & 1.033 & 0.1966 & 1.021 \\
\hline 24951 & IC $S M$ & 8.061 & 1.011 & 1.0860 & 1.040 & 0.1938 & 1.027 \\
\hline 24952 & IC SF & 8.102 & 0.997 & 1.1290 & 1.039 & 0.1966 & 1.007 \\
\hline 24953 & IC SM & 7.842 & 1.012 & 1.0480 & 1.043 & 0.1952 & 1.013 \\
\hline 24954 & IC SF & 7.828 & 1.003 & 1.0920 & 1.038 & 0.2022 & 0.999 \\
\hline 24955 & IC SM & 7.566 & 1.008 & 1.0170 & 1.035 & 0.1893 & 1.004 \\
\hline 24956 & IC SF & 7.474 & 1.006 & 1.0620 & 1.032 & 0.1840 & 0.995 \\
\hline 24957 & IC SM & 7.438 & 1.003 & 0.9942 & 1.042 & 0.1760 & 1.031 \\
\hline 24958 & IC SF & 7.572 & 0.998 & 1.0520 & 1.049 & 0.1845 & 1.018 \\
\hline 24959 & IC SF & 7.581 & 1.006 & 1.0550 & 1.050 & 0.1923 & 1.020 \\
\hline 24960 & IC SM & 7.472 & 1.016 & 0.9974 & 1.051 & 0.1862 & 1.016 \\
\hline 24961 & IC $\mathrm{SF}$ & 7.644 & 0.998 & 1.0590 & 1.049 & 0.1847 & 1.008 \\
\hline 24962 & IC SF & 7.639 & 1.000 & 1.0700 & 1.045 & 0.1863 & 0.995 \\
\hline 24963 & IC SM & 7.491 & 1.009 & 1.0020 & 1.048 & 0.1799 & 1.017 \\
\hline 24964 & IC $S F$ & 7.607 & 0.989 & 1.0610 & 1.036 & 0.1846 & 0.994 \\
\hline 24965 & IC $\mathrm{SF}$ & 7.433 & 1.000 & 1.0280 & 1.056 & 0.1816 & 1.008 \\
\hline 24966 & IC $S M$ & 7.132 & 1.010 & 0.9479 & 1.053 & 0.1741 & 1.011 \\
\hline 24967 & IC $S F$ & 7.016 & 0.996 & 0.9643 & 1.053 & 0.1656 & 1.025 \\
\hline 249.68 & IC SM & 6.640 & 1.009 & 0.8831 & 1.046 & 0.1583 & 1.028 \\
\hline 24969 & IC SF & 6.513 & 0.991 & 0.8993 & 1.038 & 0.1563 & 1.011 \\
\hline 24970 & IC SM & 6.104 & 1.007 & 0.8203 & 1.027 & 0.1457 & 1.045 \\
\hline 24971 & IC SF & 5.899 & 0.993 & 0.8084 & 1.042 & 0.1447 & 1.007 \\
\hline 24972 & IC SM & 5.520 & 1.003 & 0.7304 & 1.035 & 0.1390 & 1.006 \\
\hline 24973 & OC SF & 5.261 & 1.000 & 0.7303 & 1.029 & 0.1352 & 1.027 \\
\hline 24974 & OC SE & 4.905 & 1.003 & 0.6656 & 1.047 & 0.1338 & 1.028 \\
\hline 24975 & $O C D$ & 4.425 & 0.993 & 0.5900 & 1.046 & 0.1326 & 0.967 \\
\hline 24976 & $O C \quad S F$ & 3.808 & 1.002 & 0.5308 & 1.037 & 0.1042 & 1.007 \\
\hline 249.77 & OC SF & 3.241 & 0.988 & 0.4582 & 1.057 & 0.0807 & 0.932 \\
\hline 24978 & $\mathrm{RB}$ & -- & - & 0.3788 & 1.029 & 0.0334 & 1.142 \\
\hline 24979 & $\mathrm{RB}$ & - & $r$ & 0.2990 & 1.047 & 0.0198 & 0.991 \\
\hline 24980 & $R B$ & $\sim$ & - & 0.2311 & 1.051 & 0.0117 & 0.922 \\
\hline 24981 & $\mathrm{RB}$ & $\sim$ & $m$ & 0.1793 & 1.041 & 0.0068 & 0.895 \\
\hline 24982 & $\mathrm{RB}$ & $-\cdots$ & $-\infty$ & 0.1496 & 0.989 & 0.0036 & 0.949 \\
\hline
\end{tabular}


TABLE 4.2. ZPPR-18B: Radial ${ }^{235} \mathrm{U}$ Fission Distributions along the $x$-axis at $z=5.16 \mathrm{~cm}$

\begin{tabular}{|c|c|c|c|c|c|c|c|}
\hline \multicolumn{4}{|c|}{ Half -1 (CRP Half) $z=+5.16 \mathrm{~cm}$} & \multicolumn{4}{|c|}{ Half -2 (CR Half) $z=-5.16 \mathrm{~cm}$} \\
\hline Matrix & Zone & Exp. & $\mathrm{C} / \mathrm{E}$ & Matrix & Zone & Exp. & $\mathrm{C} / \mathrm{E}$ \\
\hline 14949 & IC SF & 9.111 & 0.996 & 24949 & IC SF & 8.791 & 1.001 \\
\hline 14950 & IC $\mathrm{SF}$ & 9.050 & 1.003 & 24950 & IC $\mathrm{SF}$ & 8.781 & 1.001 \\
\hline 14951 & IC SM & 8.833 & 1.013 & 24951 & IC SM & 8.581 & 1.009 \\
\hline 14952 & IC SF & 8.791 & 1.008 & 24952 & IC SF & 8.566 & 0.999 \\
\hline 14953 & IC SM & 8.663 & 1.006 & 24953 & IC SM & 8.339 & 1.005 \\
\hline 14954 & IC $S F$ & 8.633 & 1.001 & 24954 & IC $\mathrm{SF}$ & 8.280 & 1.000 \\
\hline 14955 & IC SM & 8.381 & 1.010 & 24955 & IC SM & 8.020 & 1.006 \\
\hline 14956 & IC $S F$ & 8.419 & 0.998 & 24956 & IC $S F$ & 7.987 & 0.999 \\
\hline 14957 & IC SM & 8.269 & 1.009 & 24957 & IC SM & 7.856 & 1.010 \\
\hline 14958 & IC $\mathrm{SF}$ & 8.384 & 1.004 & 24958 & IC $S F$ & 7.990 & 1.005 \\
\hline 14959 & IC $S F$ & 8.384 & 1.002 & 24959 & IC $\mathrm{SF}$ & 8.032 & 1.005 \\
\hline 14960 & IC SM & 8.241 & 1.009 & 24960 & IC SM & 7.896 & 1.018 \\
\hline 14961 & IC $\mathrm{SF}$ & 8.329 & 1.002 & 24961 & IC SF & 8.103 & 1.000 \\
\hline 14962 & IC $S F$ & 8.289 & 1.008 & 24962 & IC SF & 8.103 & 1.005 \\
\hline 14963 & IC SM & 8.163 & 1.009 & 24963 & IC SM & 8.004 & 1.006 \\
\hline 149.64 & IC SF & 8.222 & 0.995 & 24964 & IC $\mathrm{SF}$ & 8.047 & 0.996 \\
\hline 14965 & IC SE & 8.034 & 1.005 & 24965 & IC SF & 7.891 & 1.003 \\
\hline 14966 & IC SM & 7.680 & 1.017 & 24966 & IC SM & 7.557 & 1.013 \\
\hline 14967 & IC SF & 7.575 & 0.998 & 24967 & IC $\mathrm{SF}$ & 7.401 & 1.001 \\
\hline 14968 & IC SM & 7.206 & 1.005 & 24968 & IC SM & 7.060 & 1.004 \\
\hline 149.69 & IC SF & 6.985 & 0.998 & 24969 & IC $\mathrm{SF}$ & 6.843 & 0.995 \\
\hline 14970 & IC SM & 6.622 & 1.002 & 24970 & IC SM & 6.470 & 1.000 \\
\hline 14971 & IC $\mathrm{SF}$ & 6.382 & 0.992 & 24971 & IC SF & 6.157 & 1.001 \\
\hline 14972 & IC SM & 5.980 & 1.000 & 24972 & IC SM & 5.788 & 1.005 \\
\hline 14973 & OC SF & 5.662 & 1.005 & 24973 & $O C S F$ & 5.554 & 0.993 \\
\hline 14974 & $O C S F$ & 5.262 & 0.998 & $249 \cdot 74$ & $\mathrm{OC} S \mathrm{SF}$ & 5.137 & 0.999 \\
\hline 14975 & $O C D$ & 4.728 & 1.001 & 24975 & $O C D$ & 4.675 & 0.986 \\
\hline 14976 & $O C S F$ & 4.188 & 0.990 & 24976 & $\mathrm{OC} \mathrm{SF}$ & 4.066 & 0.994 \\
\hline 14977 & $O C S F$ & 3.629 & 0.996 & 24977 & OC SF & 3.542 & 0.995 \\
\hline 14978 & $\mathrm{RB}$ & 3.077 & 1.011 & 24978 & $\mathrm{RB}$ & 3.056 & 0.993 \\
\hline 14979 & $\mathrm{RB}$ & 2.525 & 1.002 & 249.79 & $\mathrm{RB}$ & 2.460 & 1.004 \\
\hline 14980 & $\mathrm{RB}$ & 2.017 & 1.002 & 24980 & $\mathrm{RB}$ & 1.985 & 0.996 \\
\hline 14981 & $R B$ & 1.653 & 0.997 & 24981 & $\mathrm{RB}$ & 1.604 & 1.005 \\
\hline 14982 & $\mathrm{RB}$ & 1.381 & 1.067 & 24982 & $\mathrm{RB}$ & 1.363 & 1.058 \\
\hline 14983 & $\mathrm{RR}$ & $-\pi$ & $-\infty$ & 24983 & $\mathrm{RR}$ & 1.431 & 1.106 \\
\hline
\end{tabular}




\begin{tabular}{|c|c|c|c|c|c|c|c|c|c|}
\hline \multirow[b]{3}{*}{ Matrix } & \multirow[b]{3}{*}{ Zone } & \multirow{2}{*}{\multicolumn{2}{|c|}{$\begin{array}{l}\text { Half }-1 z=+28 \mathrm{~cm} \\
2{ }^{35} U(n, f) \\
\end{array}$}} & \multicolumn{6}{|c|}{$\mathrm{HaIf}-2 \mathrm{z}=-28 \mathrm{~cm}$} \\
\hline & & & & \multicolumn{2}{|c|}{$235 U(n, f)$} & \multicolumn{2}{|c|}{$238 U(n, Y)$} & \multicolumn{2}{|c|}{$2{ }^{38} \mathrm{U}(\mathrm{n}, \mathrm{f})$} \\
\hline & & Exp. & $\mathrm{C} / \mathrm{E}$ & Exp. & $C / E$ & Exp. & $C / E$ & Exp. & $\mathrm{C} / \mathrm{E}$ \\
\hline $1,249 \quad 49$ & IC $S F$ & 8.025 & 0.984 & 6.811 & 0.996 & 0.8979 & 1.038 & 0.1494 & 1.013 \\
\hline 1,24950 & IC SF & 7.971 & 0.991 & 6.784 & 0.998 & 0.9024 & 1.031 & 0.1484 & 1.023 \\
\hline 1,24951 & IC SM & 7.793 & 1.001 & 6.618 & 1.005 & 0.8407 & 1.031 & 0.1471 & 1.020 \\
\hline $1,249.52$ & IC $\mathrm{SF}$ & 7.809 & 0.993 & 6.546 & 1.001 & 0.8594 & 1.046 & 0.1480 & 1.007 \\
\hline $1,249 \quad 53$ & IC SM & 7.694 & 0.995 & 6.333 & 1.008 & 0.8032 & 1.037 & 0.1471 & 1.007 \\
\hline $1,249 \quad 54$ & IC SF & 7.761 & 0.983 & 6.288 & 0.996 & 0.8307 & 1.035 & 0.1524 & 0.989 \\
\hline 1,24955 & IC SM & 7.552 & 0.996 & 6.020 & 1.008 & 0.7573 & 1.047 & 0.1391 & 1.014 \\
\hline 1,24956 & IC SF & 7.586 & 0.989 & 5.976 & 1.001 & 0.7950 & 1.036 & 0.1371 & 0.988 \\
\hline 1,24957 & IC SM & 7.460 & 0.999 & 5.895 & 1.010 & 0.7410 & 1.051 & 0.1288 & 1.044 \\
\hline 1,24958 & IC SF & 7.537 & 0.991 & 6.069 & 1.000 & 0.8052 & 1.037 & 0.1392 & 1.006 \\
\hline $1,249.59$ & IC $S F$ & 7.421 & 0.996 & 6.126 & 1.004 & 0.8037 & 1.051 & 0.1445 & 1.019 \\
\hline 1,24960 & IC SM & 7.209 & 1.007 & 6.055 & 1.020 & 0.7733 & 1.042 & 0.1430 & 1.000 \\
\hline $1,249 \quad 61$ & IC $S F$ & 7.286 & 0.992 & 6.268 & 1.002 & 0.8355 & 1.033 & 0.1451 & 0.977 \\
\hline 1,24962 & IC $\mathrm{SF}$ & 7.283 & 0.988 & 6.366 & 0.998 & 0.8499 & 1.027 & 0.1431 & 0.992 \\
\hline $1,249 \quad 63$ & IC SM & 7.109 & 0.993 & 6.200 & 1.018 & 0.7869 & 1.045 & 0.1387 & 1.015 \\
\hline 1,24964 & IC SF & 7.093 & 0.985 & 6.340 & 0.994 & 0.8440 & 1.025 & 0.1420 & 0.996 \\
\hline $1,249 \quad 65$ & IC $S F$ & 6.958 & 0.990 & 6.201 & 1.005 & 0.8232 & 1.040 & 0.1398 & 1.012 \\
\hline 1,24966 & IC SM & 6.612 & 1.006 & 6.033 & 0.999 & 0.7512 & 1.046 & 0.1334 & 1.019 \\
\hline 1,24967 & IC $S F$ & 6.517 & 0.989 & 5.843 & 0.997 & 0.7717 & 1.036 & 0.1309 & 1.000 \\
\hline 1,24968 & IC SM & 6.234 & 0.991 & 5.543 & 1.004 & 0.6974 & 1.040 & 0.1242 & 1.009 \\
\hline 1,24969 & IC SF & 6.052 & 0.984 & 5.355 & 0.995 & 0.7074 & 1.033 & 0.1222 & 0.993 \\
\hline 1,24970 & IC SM & 5.701 & 0.996 & 5.028 & 1.003 & 0.6344 & 1.036 & 0.1156 & 1.008 \\
\hline 1,24971 & IC SF & 5.487 & 0.989 & 4.778 & 1.002 & 0.6264 & 1.046 & 0.1095 & 1.014 \\
\hline 1,24972 & IC SM & 5.128 & 1.000 & 4.476 & 1.007 & 0.5652 & 1.035 & 0.1048 & 1.014 \\
\hline $1,249 \quad 73$ & OC SF & 4.922 & 0.991 & 4.241 & 1.005 & 0.5597 & 1.039 & 0.1065 & 0.990 \\
\hline 1,24974 & $O C S F$ & 4.560 & 0.985 & 3.947 & 1.003 & 0.5105 & 1.055 & 0.1057 & 0.987 \\
\hline 1,24975 & $O C D$ & 4.107 & 0.984 & 3.552 & 1.001 & 0.4578 & 1.040 & 0.1037 & 0.939 \\
\hline 1,24976 & OC SF & 3.624 & 0.975 & 3.150 & 0.990 & 0.4132 & 1.030 & 0.0810 & 0.984 \\
\hline $1,249 \quad 77$ & OC SF & 3.152 & 0.977 & 2.729 & 0.999 & 0.3519 & 1.065 & 0.0605 & 0.945 \\
\hline 1,24978 & $\mathrm{RB}$ & 2.647 & 1.002 & 2.323 & 1.014 & -- & - & +2 & -+ \\
\hline 1,24979 & $\mathrm{RB}$ & 2.193 & 0.984 & 1.924 & 1.000 & -- & $-\infty$ & $\sim$ & $m$ \\
\hline 1,24980 & $\mathrm{RB}$ & 1.716 & 1.005 & 1.546 & 0.998 & $\ldots$ & $\cdots$ & $\therefore-$ & $\ldots$ \\
\hline 1,24981 & $\mathrm{RB}$ & 1.417 & 0.993 & 1.270 & 0.994 & $\ldots$ & $m$ & -- & $\ldots$ \\
\hline $1,249 \quad 82$ & $\mathrm{RB}$ & 1.205 & 1.044 & 1.052 & 1.077 & r & بنز & $-r$ & $-\infty$ \\
\hline
\end{tabular}


TABLE 4.4 ZPPR-18B: Radial

$$
\begin{gathered}
2{ }^{35} \mathrm{U}(\mathrm{n}, \mathrm{f}) \text { Distribution } \\
\text { along the } \mathrm{y} \text {-axis at } \\
z=-5.16 \mathrm{~cm}
\end{gathered}
$$

\begin{tabular}{lllll}
\hline & & & & \\
& & & & \\
\cline { 4 - 5 } Matrix $(\mathrm{n}, \mathrm{f})$ \\
\hline
\end{tabular}


TABLE 4.5 ZPPR-18B: Radial Reaction Rate Distributions at $15^{\circ}$ to the $\mathrm{x}$-axis at $\mathrm{z}=-5.16 \mathrm{~cm}$

\begin{tabular}{|c|c|c|c|c|c|c|c|}
\hline \multirow[b]{2}{*}{ Matrix } & \multirow[b]{2}{*}{ Zone } & \multicolumn{2}{|c|}{${ }^{2}{ }^{3}{ }^{4} \mathrm{Pu}(\mathrm{n}, \mathrm{f})$} & \multicolumn{2}{|c|}{$2{ }^{2} \mathrm{\cup} \cup(n, y)$} & \multicolumn{2}{|c|}{$23^{8} U(n, f)$} \\
\hline & & Exp. & $C / E$ & Exp. & $\mathrm{C} / \mathrm{E}$ & Exp. & $C / E$ \\
\hline 24850 & IC SF & 8.278 & 0.994 & 1.1610 & 1.033 & 0.1988 & 1.007 \\
\hline 24852 & IC SM & 7.959 & 1.010 & 1.0620 & 1.044 & 0.1935 & 1.021 \\
\hline 24853 & IC $S F$ & 7.965 & 0.998 & 1.0950 & 1.051 & 0.1980 & 1.008 \\
\hline 24754 & IC $S F$ & 7.652 & 1.005 & 1.0620 & 1.046 & 0.1915 & 1.000 \\
\hline 24755 & IC SM & $m-$ & $-\infty$ & $-n$ & $m-$ & $-\div$ & $-\infty$ \\
\hline 24756 & IC $S F$ & 7.316 & 0.994 & 1.0220 & 1.036 & 0.1769 & 0.983 \\
\hline 24757 & IC $S F$ & 7.296 & 0.991 & 0.9996 & 1.054 & 0.1748 & 0.987 \\
\hline 24657 & IC SM & 6.823 & 0.990 & 0.9026 & 1.032 & 0.1571 & 0.988 \\
\hline 24658 & IC SF & 6.898 & 1.013 & 0.9501 & 1.069 & 0.1647 & 1.001 \\
\hline 24659 & IC $S M$ & 7.070 & 1.018 & 0.9438 & 1.052 & 0.1699 & 1.017 \\
\hline 24660 & IC SF & 7.383 & 0.997 & 1.0180 & 1.050 & 0.1758 & 1.010 \\
\hline 24560 & IC SM & 7.134 & 1.011 & 0.9536 & 1.047 & 0.1707 & 1.022 \\
\hline 24561 & IC $S F$ & 7.481 & 0.983 & 1.0130 & 1.056 & 0.1802 & 1.007 \\
\hline 24562 & IC SM & 7.287 & 1.009 & 0.9783 & 1.038 & 0.1779 & 1.001 \\
\hline 24463 & IC SM & 7.181 & 1.007 & 0.9569 & 1.046 & 0.1713 & 1.007 \\
\hline 24464 & IC $S F$ & 7.227 & 0.992 & 1.0030 & 1.043 & 0.1728 & 0.987 \\
\hline 24465 & IC SM & 7.020 & 1.003 & 0.9297 & 1.046 & 0.1633 & 1.034 \\
\hline $244 \quad 56$ & IC SF & 6.946 & 0.994 & 0.9672 & 1.038 & 0.1665 & 1.009 \\
\hline 24367 & IC $S F$ & 6.535 & 1.003 & 0.9139 & 1.041 & 0.1619 & 0.988 \\
\hline 24368 & IC SF & 6.307 & 0.990 & 0.8749 & 1.033 & 0.1532 & 1.009 \\
\hline 24369 & IC $S M$ & 5.747 & 1.003 & 0.7642 & 1.036 & 0.1397 & 1.003 \\
\hline 24370 & IC SM & 5.100 & 1.018 & 0.7129 & 1.048 & 0.1241 & 0.995 \\
\hline 24270 & IC SM & 4.814 & 1.028 & 0.6544 & 1.045 & 0.1193 & 0.991 \\
\hline & $\mathrm{CR}$ & & & & & & \\
\hline 24273 & OC SF & 3.933 & 1.015 & 0.5495 & 1.043 & 0.1035 & 0.992 \\
\hline 24173 & OC SF & 3.924 & 1.013 & 0.5529 & 1.038 & 0.1026 & 1.029 \\
\hline 24174 & $O C S F$ & 3.895 & 1.011 & 0.5389 & 1.043 & 0.1071 & 1.009 \\
\hline 24175 & $O C D$ & 3.684 & 0.989 & 0.4998 & 1.029 & 0.1118 & 0.931 \\
\hline 24176 & OC SF & 3.283 & 0.988 & 0.4501 & 1.034 & 0.0905 & 0.992 \\
\hline 24077 & OC SF & 2.675 & 0.994 & 0.3814 & 1.036 & 0.0698 & 0.940 \\
\hline 24078 & $\mathrm{RB}$ & - & $\rightarrow$ & 0.3053 & 1.039 & 0.0288 & 1.136 \\
\hline 24079 & $\mathrm{RB}$ & $-\pi$ & $\rightarrow$ & 0.2500 & 1.020 & 0.0179 & 0.927 \\
\hline 13980 & $\mathrm{RB}$ & $-m$ & $\cdots$ & 0.1812 & 1.042 & 0.0094 & 0.903 \\
\hline 23981 & $\mathrm{RB}$ & - & $m$ & 0.1386 & 1.040 & 0.0059 & 0.803 \\
\hline 23982 & $\mathrm{RB}$ & - & $m$ & 0.1164 & 0.977 & 0.0027 & 1.006 \\
\hline
\end{tabular}


TABLE 4.6 ZPPR 18B: Radial ${ }^{235} \mathrm{U}$ Fission Distributions at $15^{\circ}$ to the $x$-axis at $z=5.16 \mathrm{~cm}$

\begin{tabular}{|c|c|c|c|c|c|c|c|}
\hline Half -1 & \multicolumn{3}{|c|}{ (CRP Half) $z=+5.16 \mathrm{~cm}$} & \multicolumn{4}{|c|}{ Half -2 (CR Half) $z=-5.16 \mathrm{~cm}$} \\
\hline Matrix & Zone & Exp. & $\mathrm{C} / \mathrm{E}$ & Matrix & Zone & Exp. & $\mathrm{C} / \mathrm{E}$ \\
\hline $148 \quad 50$ & IC SF & 8.988 & 1.001 & 24850 & IC SF & 8.644 & 1.007 \\
\hline 14852 & IC SM & 8.722 & 1.011 & 24852 & IC $S M$ & 8.400 & 1.012 \\
\hline 14853 & IC SF & 8.697 & 1.002 & 24853 & IC SF & 8.338 & 1.003 \\
\hline 14754 & IC SF & 8.545 & 0.993 & 24754 & IC SF & 8.069 & 0.995 \\
\hline $147 \cdot 55$ & IC SM & 8.184 & 1.015 & 24755 & IC SM & me & - \\
\hline 14756 & IC $\mathrm{SF}$ & 8.301 & 0.993 & 247.56 & IC SF & 7.590 & 0.993 \\
\hline 14757 & IC SF & 8.227 & 0.995 & 24757 & IC SF & 7.492 & 0.999 \\
\hline 14658 & IC SF & 8.045 & 1.000 & $246 \quad 58$ & IC SF & 7.139 & 1.012 \\
\hline 14659 & IC SM & 7.977 & 1.011 & 24659 & IC SM & 7.407 & 1.018 \\
\hline 14660 & IC SF & 8.129 & 0.999 & 24660 & IC $S F$ & 7.773 & 0.997 \\
\hline 14560 & IC SM & 7.996 & 1.006 & 24560 & IC SM & 7.541 & 1.012 \\
\hline 14561 & IC SF & 8.155 & 0.990 & 24561 & IC SF & 7.768 & 1.002 \\
\hline 14562 & IC SM & 7.997 & 1.002 & 24562 & IC SM & 7.700 & 1.010 \\
\hline 14463 & IC SM & 7.880 & 1.001 & 24463 & IC SM & 7.626 & 1.008 \\
\hline 14464 & IC SF & 7.802 & 0.999 & 24464 & IC SF & 7.684 & 0.991 \\
\hline 14465 & IC SM & 7.550 & 1.011 & 24465 & IC SM & 7.425 & 1.004 \\
\hline 14466 & IC SF & 7.531 & 0.992 & $244 \quad 66$ & IC SF & 7.325 & 0.995 \\
\hline 143.67 & IC SF & 7.151 & 0.994 & 24367 & IC $\mathrm{SF}$ & 6.966 & 0.990 \\
\hline 14368 & IC SF & 6.911 & 0.987 & 24368 & IC SF & 6.579 & 0.995 \\
\hline 14369 & IC SM & 6.397 & 1.002 & 24369 & IC SM & 5.988 & 1.006 \\
\hline 14370 & IC SF & 6.057 & 0.990 & 24370 & IC SF & 5.189 & 1.029 \\
\hline \multirow[t]{2}{*}{14270} & IC SM & 5.957 & 0.988 & 24270 & IC SM & 5.025 & 1.032 \\
\hline & $\mathrm{CRP}$ & & & $\mathrm{CR}$ & & & \\
\hline 14273 & OC SF & 4.998 & 0.995 & 24273 & OC SF & 4.027 & 1.029 \\
\hline 14173 & OC SF & 4.968 & 0.987 & 24173 & OC SF & 4.170 & 1.010 \\
\hline 14174 & OC SF & 4.570 & 0.990 & 24174 & $\mathrm{OC} \mathrm{SF}$ & 4.091 & 1.007 \\
\hline 14175 & $O C D$ & 4.142 & 0.987 & 24175 & $O C D$ & 3.840 & 0.995 \\
\hline 14176 & OC SF & 3.602 & 0.991 & 24176 & $\mathrm{OC} \quad \mathrm{SF}$ & 3.438 & 0.987 \\
\hline $140 \quad 77$ & $O C S F$ & 3.055 & 0.972 & $240: 77$ & OC SF & 2.904 & 0.982 \\
\hline 14078 & $\mathrm{RB}$ & 2.571 & 0.985 & 24078 & $\mathrm{RB}$ & 2.492 & 0.980 \\
\hline 14079 & RB & 2.060 & 1.000 & 24079 & $\mathrm{RB}$ & 2.047 & 0.973 \\
\hline 13980 & $\mathrm{RB}$ & 1.591 & 0.983 & 23980 & $\mathrm{RB}$ & 1.561 & 0.973 \\
\hline 13981 & RB & 1.256 & 1.008 & 23981 & $\mathrm{RB}$ & 1.251 & 0.985 \\
\hline 1.3982 & $\mathrm{RB}$ & 1.068 & 1.052 & $239 \quad 82$ & $\mathrm{RB}$ & 1.043 & 1.050 \\
\hline
\end{tabular}


TABLE 4.7 ZPPR-18B: Radial Reaction Rate Distributions at $15^{\circ}$ to the $x-a x i s$ at $z=28.02 \mathrm{~cm}$

\begin{tabular}{|c|c|c|c|c|c|c|c|c|c|}
\hline \multirow[b]{3}{*}{ Matrix } & \multirow[b]{3}{*}{ Zone } & \multirow{2}{*}{\multicolumn{2}{|c|}{$\begin{array}{l}\mathrm{Half}-1 \mathrm{z}=+28 \mathrm{~cm} \\
23 \mathrm{~s} U(\mathrm{n}, \mathrm{f}) \\
\end{array}$}} & \multicolumn{6}{|c|}{ Half $-2 z=-28 \mathrm{~cm}$} \\
\hline & & & & \multicolumn{2}{|c|}{$2^{35} U(n, f)$} & \multicolumn{2}{|c|}{${ }^{23} \mathrm{U} U(n, \gamma)$} & \multicolumn{2}{|c|}{$2^{3} \mathrm{U} U(\mathrm{n}, \mathrm{f})$} \\
\hline & & Exp. & $\mathrm{C} / \mathrm{E}$ & Exp. & $\mathrm{C} / \mathrm{E}$ & Exp. & $\mathrm{C} / \mathrm{E}$ & Exp. & $\mathrm{C} / \mathrm{E}$ \\
\hline 1,24850 & IC SF & - & $m$ & 6.689 & 1.001 & 0.8839 & 1.045 & 0.1520 & 0.995 \\
\hline $1,248 \quad 52$ & IC SM & 7.741 & 0.998 & 6.427 & 1.011 & 0.8082 & 1.050 & 0.1460 & 1.016 \\
\hline 1,24853 & IC $\mathrm{SF}$ & 7.736 & 0.992 & 6.320 & 1.005 & 0.8340 & 1.050 & 0.1459 & 1.024 \\
\hline 1,24754 & IC SE & 7.729 & 0.983 & 6.029 & 0.996 & 0.8066 & 1.034 & 0.1416 & 1.002 \\
\hline 1,24755 & IC SM & 7.555 & 1.000 & - & $\cdots$ & $-r$ & $-\infty$ & $\sim$ & $\ldots$ \\
\hline 1,24756 & IC SF & 7.610 & 0.999 & 5.542 & 0.991 & 0.7487 & 1.042 & 0.1319 & 0.965 \\
\hline $1,247 \quad 57$ & IC $\mathrm{SF}$ & 7.620 & 0.991 & 5.446 & 1.002 & 0.7453 & 1.044 & 0.1295 & 0.977 \\
\hline $1,246 / 57$ & IC SM & 7.676 & 1.001 & 4.742 & 0.994 & 0.6444 & 1.032 & 0.1148 & 0.975 \\
\hline 1,24658 & IC $\mathrm{SF}$ & 7.651 & 0.981 & 5.181 & 1.007 & 0.7050 & 1.053 & 0.1171 & 1.029 \\
\hline 1,24659 & IC $S M$ & 7.273 & 1.004 & 5.495 & 1.021 & 0.7113 & 1.044 & 0.1239 & 1.033 \\
\hline $1,246 \quad 60$ & IC SF & - & $\sim$ & 5.836 & 1.008 & - & - & $\ldots$ & $-\cdots$ \\
\hline 1,24560 & IC SM & 7.100 & 1.008 & 5.621 & 1.027 & 0.7238 & 1.045 & 0.1305 & 1.026 \\
\hline 1,24561 & IC SF & 7.113 & 0.994 & 5.929 & 1.009 & - & -- & $-\infty$ & $\ldots$ \\
\hline $1,245 \quad 62$ & IC SM & 6.933 & 1.001 & 5.990 & 1.009 & 0.7683 & 1.027 & 0.1358 & 1.000 \\
\hline 1,24463 & IC SM & 6.775 & 1.004 & 5.966 & 1.008 & 0.7527 & 1.039 & 0.1314 & 1.007 \\
\hline $1,244.64$ & IC SF & 6.764 & 0.991 & 5.963 & 1.002 & $\rightarrow$ & -- & $-r$ & $r$ \\
\hline 1,24465 & IC SM & 6.536 & 1.003 & 5.818 & 1.005 & 0.7291 & 1.046 & 0.1296 & 1.002 \\
\hline 1,24466 & IC $\mathrm{SF}$ & 6.443 & 0.997 & 5.716 & 0.997 & -- & $\rightarrow$ & -- & -- \\
\hline 1,24367 & IC SF & 6.280 & 0.981 & 5.350 & 0.999 & 0.7164 & 1.031 & 0.1208 & 1.009 \\
\hline 1,24368 & IC SF & 6.071 & 0.983 & 5.047 & 0.993 & 0.6685 & 1.038 & 0.1178 & 0.992 \\
\hline 1,24369 & IC SF & 5.795 & 0.989 & 4.470 & 1.011 & 0.5712 & 1.045 & 0.1024 & 1.021 \\
\hline 1,24370 & IC SF & 5.666 & 0.984 & 3.754 & 1.027 & 0.5144 & 1.058 & 0.0964 & 0.937 \\
\hline 1,24270 & $\begin{array}{l}\text { IC SM } \\
\text { CRP/CR }\end{array}$ & 5.631 & 0.980 & 3.643 & 1.018 & 0.4733 & 1.041 & 0.0850 & 1.009 \\
\hline 1,24273 & $\mathrm{OC} \quad \mathrm{SF}$ & 4.908 & 0.979 & 2.736 & 1.028 & 0.3686 & 1.060 & 0.0734 & 0.982 \\
\hline 1,24173 & OC SF & 4.789 & 0.974 & 2.891 & 1.006 & 0.3790 & 1.043 & 0.0757 & 0.985 \\
\hline 1,24174 & OC SF & $\cdots$ & $\cdots$ & 2.930 & 1.006 & 0.3844 & 1.046 & 0.0751 & 1.032 \\
\hline 1,24175 & $O C D$ & بs- & $r$ & 2.807 & 0.996 & 0.3625 & 1.037 & 0.0803 & 0.940 \\
\hline 1,24176 & OC SF & -- & $\rightarrow$ & 2.554 & 0.987 & 0.3360 & 1.030 & 0.0661 & 0.995 \\
\hline 1,24077 & OC SF & $-m$ & $m$ & 2.200 & 0.975 & 0.2873 & 1.034 & 0.0516 & 0.940 \\
\hline 14078 & $R B$ & $\cdots$ & $m$ & 1.870 & 0.992 & - & - & $-r$ & -- \\
\hline 14079 & $\mathrm{RB}$ & $m$ & $-r$ & 1.524 & 0.999 & $-m$ & - & $-\infty$ & -- \\
\hline 13980 & $\mathrm{RB}$ & - & -- & 1.191 & 0.982 & - & -- & $\sim$ & -- \\
\hline 13981 & RB & $-r$ & - & 0.945 & 1.009 & $m$ & -. & $-\infty$ & $-\infty$ \\
\hline 13982 & $\mathrm{RB}$ & $\cdots$ & $\sim$ & 0.784 & 1.087 & $-\infty$ & -- & $\cdots$ & -- \\
\hline
\end{tabular}


TABLE 4.8 ZPPR-18B: Radial Reaction Rate Distributions at $30^{\circ}$ to the $x$-axis

\begin{tabular}{|c|c|c|c|c|c|c|c|c|c|}
\hline \multirow[b]{3}{*}{ Matrix } & \multirow[b]{3}{*}{ Zone } & \multirow{2}{*}{\multicolumn{2}{|c|}{$\begin{array}{l}\mathrm{HaIf}-2 \mathrm{z}=-28 \mathrm{~cm} \\
235 \mathrm{U}(\mathrm{n}, \mathrm{f})\end{array}$}} & \multicolumn{6}{|c|}{$\mathrm{Half}-2 \mathrm{z}=-5 \mathrm{~cm}$} \\
\hline & & & & \multicolumn{2}{|c|}{$23^{5} U(n, f)$} & \multicolumn{2}{|c|}{${ }^{23} \mathrm{U}(n, Y)$} & \multicolumn{2}{|c|}{${ }^{23} \mathrm{U} U(\mathrm{n}, \mathrm{f})$} \\
\hline & & Exp. & $C / E$ & Exp. & $C / E$ & Exp. & $\mathrm{C} / \mathrm{E}$ & Exp. & $\mathrm{C} / \mathrm{E}$ \\
\hline 24654 & IC SM & 5.691 & 1.013 & 7.721 & 1.010 & 0.9852 & 1.042 & 0.1762 & 1.023 \\
\hline $245 \quad 55$ & $\begin{array}{l}\text { IC SM } \\
\text { CR }\end{array}$ & 4.717 & 1.040 & 6.690 & 1.044 & 0.8869 & 1.050 & 0.1613 & 0.968 \\
\hline 24458 & IC SM & 4.538 & 1.025 & 6.446 & 1.033 & 0.8405 & 1.049 & 0.1425 & 1.020 \\
\hline 24358 & IC SE & 4.822 & 1.014 & 6.784 & 1.012 & 0.8997 & 1.048 & 0.1556 & 0.959 \\
\hline 24359 & IC SM & 5.283 & 1.026 & 7.199 & 1.017 & 0.9153 & 1.043 & 0.1605 & 1.017 \\
\hline $242 \quad 60$ & IC SM & 5.705 & 1.020 & 7.491 & 1.016 & 0.9515 & 1.040 & 0.1682 & 1.019 \\
\hline 24261 & IC SF & 5.987 & 1.000 & 7.736 & 0.999 & 1.0200 & 1.040 & 0.1734 & 0.996 \\
\hline 24262 & IC SF & 6.103 & 1.001 & 7.775 & 1.004 & 1.0310 & 1.034 & 0.1680 & 0.994 \\
\hline 24162 & $\begin{array}{l}\text { IC SE } \\
\text { CRP }\end{array}$ & 6.108 & 1.007 & 7.814 & 1.004 & 1.0470 & 1.023 & 0.1631 & 0.980 \\
\hline 24065 & IC SM & 5.806 & 0.998 & 7.365 & 1.003 & 0.9252 & 1.028 & 0.1530 & 0.967 \\
\hline 23965 & IC SF & 5.704 & 0.988 & 7.267 & 0.994 & 0.9758 & 1.018 & 0.1555 & 0.973 \\
\hline 23966 & IC SM & 5.199 & 1.002 & 6.765 & 0.996 & 0.8518 & 1.031 & 0.1509 & 1.006 \\
\hline 23867 & IC SM & 4.722 & 1.001 & 6.194 & 1.003 & 0.7852 & 1.037 & 0.1410 & 1.035 \\
\hline $238 \quad 68$ & IC SF & 4.609 & 0.985 & 6.046 & 0.994 & 0.7994 & 1.035 & 0.1432 & 1.005 \\
\hline 23768 & IC SM & 4.353 & 0.991 & 5.737 & 1.006 & 0.7431 & 1.020 & 0.1383 & 1.027 \\
\hline
\end{tabular}


TABLE 4.9 ZPPR 18B: Axial Reaction Rate Distributions in Matrix 249-49 (core center)

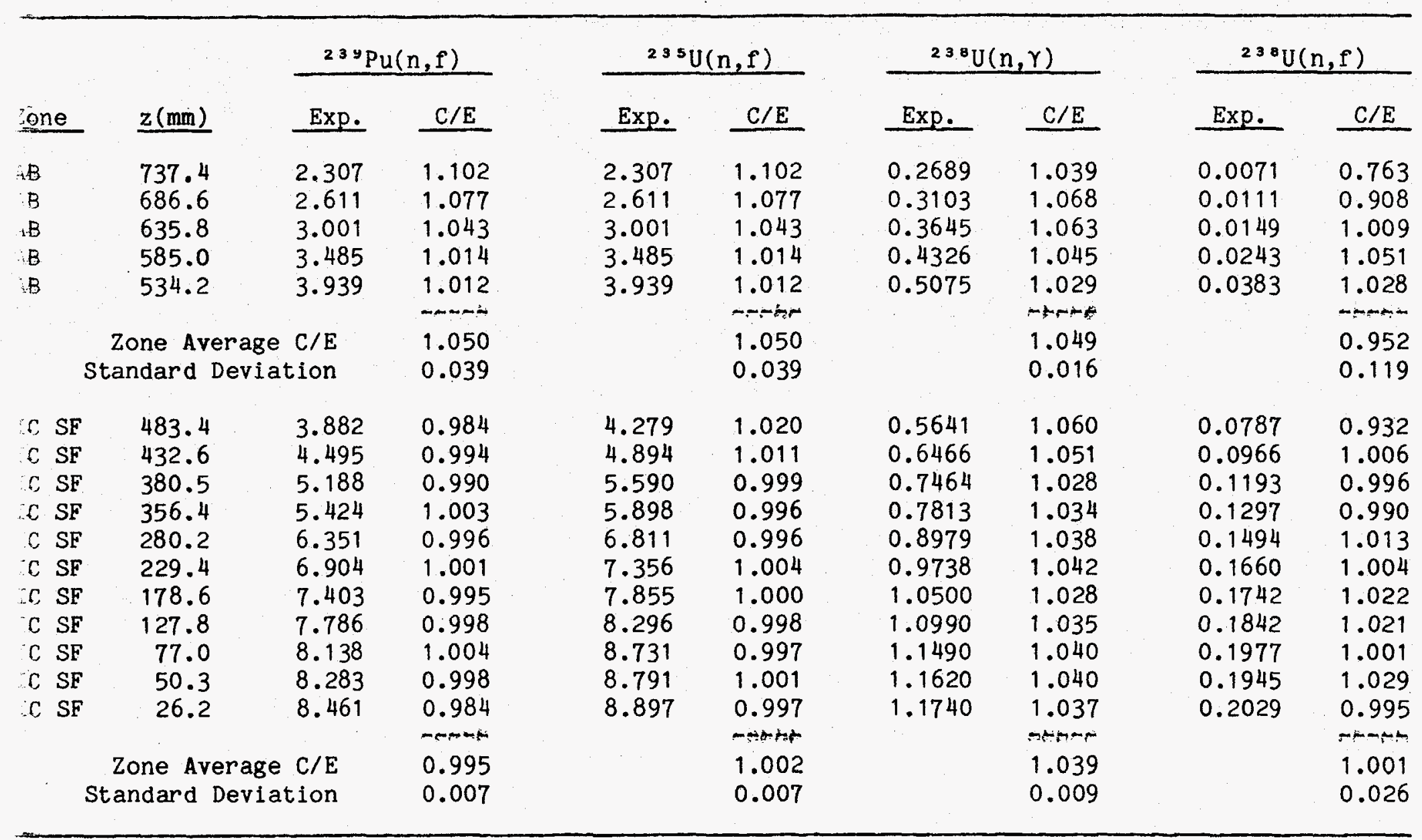


TABLE 4.10 ZPPR 18B: Axial Reaction Rate Distributions in Matrix 149-49 (core center)

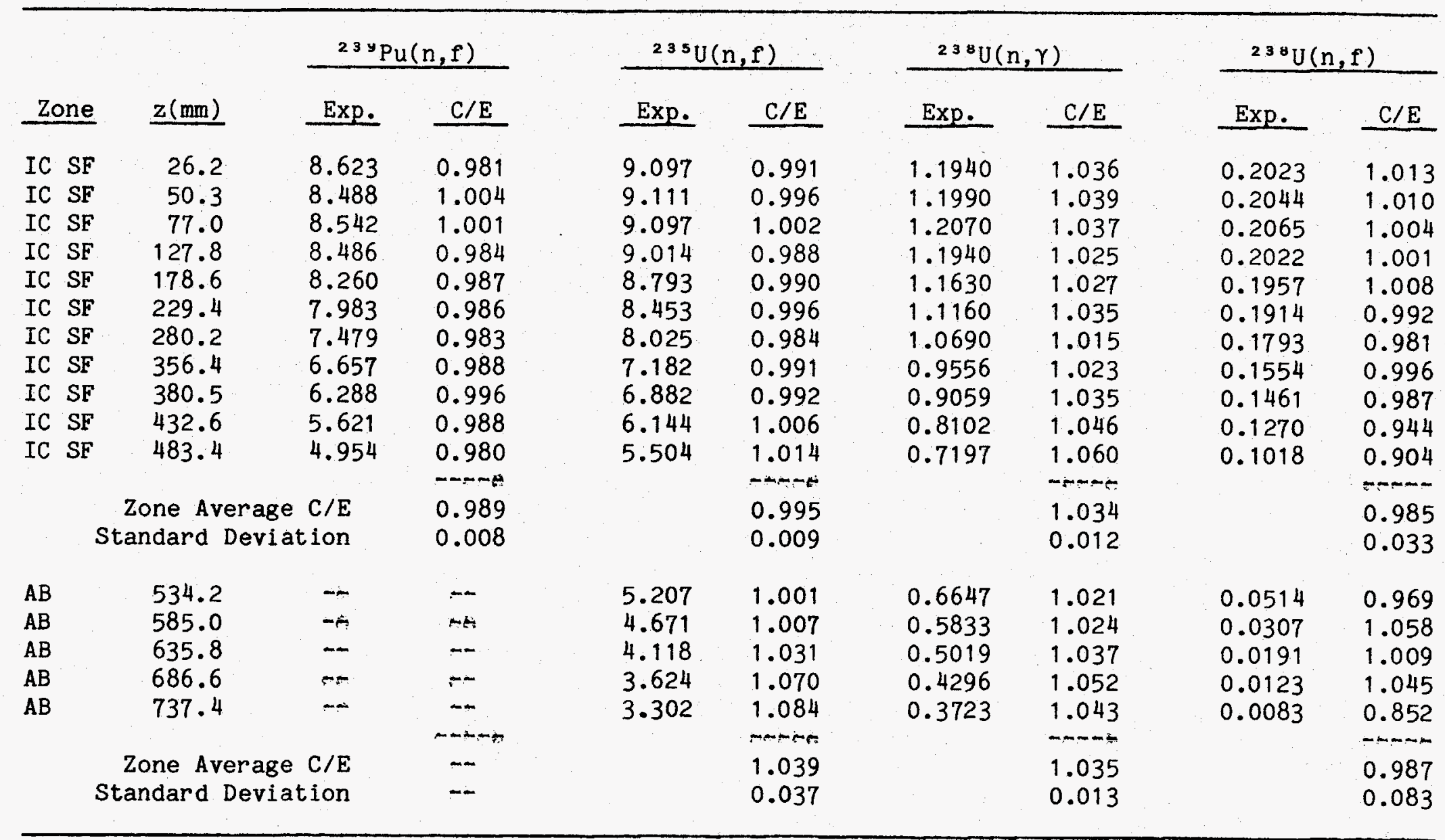


TABLE 4.11 ZPPR-18B: Axial ${ }^{235} \mathrm{U}$ Fission Distributions in Matrix 249-64 and 149m-64

\begin{tabular}{|c|c|c|c|c|c|c|c|}
\hline \multirow[b]{2}{*}{ Zone } & & \multicolumn{2}{|c|}{ Matrix 249-64 } & \multirow[b]{2}{*}{ Zone } & \multirow[b]{2}{*}{$\underline{z(m m)}$} & \multicolumn{2}{|c|}{ Matrix 149-64 } \\
\hline & $\underline{z(m m)}$ & Exp. & $\mathrm{C} / \mathrm{E}$ & & & Exp. & $\mathrm{C} / \mathrm{E}$ \\
\hline$A B$ & 737.4 & 2.233 & 1.097 & $A B$ & 737.4 & 2.861 & 1.085 \\
\hline$A B$ & 686.6 & 2.498 & 1.080 & $A B$ & 686.6 & 3.185 & 1.058 \\
\hline$A B$ & 635.8 & 2.908 & 1.029 & $A B$ & 635.8 & 3.638 & 1.016 \\
\hline$A B$ & 585.0 & 3.350 & 1.005 & $A B$ & 585.0 & 4.075 & 1.006 \\
\hline \multirow[t]{3}{*}{$A B$} & 534.2 & 3.765 & 1.005 & $A B$ & 534.2 & 4.545 & 1.002 \\
\hline & & & $-4 m a r$ & & & & m-ran \\
\hline & \multicolumn{2}{|c|}{$\begin{array}{l}\text { Zone Average C/E } \\
\text { Standard Deviation }\end{array}$} & $\begin{array}{l}1.043 \\
0.043\end{array}$ & \multicolumn{3}{|c|}{$\begin{array}{l}\text { Zone Average C/E } \\
\text { Standard Deviation }\end{array}$} & $\begin{array}{l}1.033 \\
0.036\end{array}$ \\
\hline IC SF & 483.4 & 4.062 & 1.014 & $A B$ & 483.4 & 4.812 & 1.014 \\
\hline IC SF & 432.6 & 4.622 & 1.006 & IC SF & 432.6 & 5.445 & 0.995 \\
\hline IC $S F$ & 381.8 & 5.263 & 0.991 & IC $\mathrm{SF}$ & 381.8 & 5.996 & 0.998 \\
\hline IC SF & 331.0 & 5.802 & 0.993 & IC SF & 331.0 & 6.605 & 0.985 \\
\hline IC $\mathrm{SF}$ & 280.2 & 6.340 & 0.994 & IC SF & 280.2 & 7.093 & 0.985 \\
\hline IC SF & 229.4 & 6.793 & 1.006 & IC SF & 229.4 & 7.539 & 0.991 \\
\hline IC SF & 178.6 & 7.281 & 0.994 & IC $\mathrm{SF}$ & 178.6 & 7.832 & 0.991 \\
\hline IC $S F$ & 127.8 & 7.673 & 0.989 & IC $S F$ & 127.8 & 8.089 & 0.986 \\
\hline IC SF & 77.0 & 7.922 & 1.002 & IC SF & 77.0 & 8.136 & 1.007 \\
\hline IC SF & 50.3 & 8.047 & 0.996 & IC SF & 50.3 & 8.222 & 0.995 \\
\hline \multirow[t]{4}{*}{ IC SF } & 26.2 & 8.106 & 0.993 & IC SF & 26.2 & 8.194 & 0.993 \\
\hline & & & $\rightarrow m-$ & & & & r- r- \\
\hline & one Ave & $\mathrm{C} / \mathrm{E}$ & 0.998 & & one Ave & $C / E$ & 0.995 \\
\hline & Adard D & tion & 0.008 & & ndard $D$ & tion & 0.009 \\
\hline
\end{tabular}


TABLE 4.12 ZPPR 18B: Axial Reaction Rate Distributions in Matrix 249-75 (outer core)

\begin{tabular}{|c|c|c|c|c|c|c|c|}
\hline \multirow[b]{2}{*}{ Zone } & \multirow[b]{2}{*}{$\underline{z}(\mathrm{~mm})$} & \multicolumn{2}{|c|}{${ }^{235} U(n, f)$} & \multicolumn{2}{|c|}{$2^{38} U(n, Y)^{a}$} & \multicolumn{2}{|c|}{$238 \mathrm{U}(\mathrm{n}, \mathrm{f})$} \\
\hline & & Exp. & $\mathrm{C} / \mathrm{E}$ & Exp. & $\mathrm{C} / \mathrm{E}$ & Exp. & $\mathrm{C} / \mathrm{E}$ \\
\hline$A B$ & 737.4 & 1.171 & 1.072 & 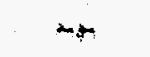 & $\omega$ & $\rightarrow$ & $\rightarrow$ \\
\hline$A B$ & 686.6 & 1.310 & 1.066 & - & 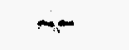 & 0.0062 & 0.920 \\
\hline$A B$ & 635.8 & 1.509 & 1.036 & $-r^{2}$ & $\rightarrow$ & 0.0097 & 0.892 \\
\hline$A B$ & 585.0 & 1.752 & 1.012 & $\rightarrow$ & $\therefore$ & 0.0142 & 1.062 \\
\hline \multirow[t]{2}{*}{$A B$} & 534.2 & 1.999 & 1.005 & $m$ & $\rightarrow$ & 0.0253 & 0.933 \\
\hline & \multicolumn{2}{|c|}{$\begin{array}{l}\text { Zone Average C/E } \\
\text { tandard Deviation }\end{array}$} & $\begin{array}{l}1.038 \\
0.030\end{array}$ & & & & $\begin{array}{l}0.952 \\
0.075\end{array}$ \\
\hline OC D & 483.4 & 2.185 & 1.016 & 0.2785 & 1.067 & 0.0520 & 0.887 \\
\hline$O C D$ & 432.6 & 2.499 & 1.018 & 0.3197 & 1.068 & 0.0648 & 0.956 \\
\hline$O C D$ & 381.8 & 2.899 & 0.998 & 0.3705 & 1.047 & 0.0791 & 0.958 \\
\hline$O C D$ & 331.0 & 3.225 & 1.001 & 0.4213 & 1.027 & 0.0931 & 0.939 \\
\hline$O C D$ & 280.2 & 3.552 & 1.001 & 0.4578 & 1.040 & 0.1037 & 0.939 \\
\hline$O C D$ & 229.4 & 3.914 & 0.991 & 0.5069 & 1.025 & 0.1119 & 0.959 \\
\hline$O C D$ & 178.6 & 4.141 & 0.996 & 0.5348 & 1.033 & 0.1196 & 0.957 \\
\hline OC D & 127.8 & 4.402 & 0.987 & 0.5648 & 1.029 & 0.1274 & 0.947 \\
\hline$O C D$ & 77.0 & 4.576 & 0.997 & 0.5814 & 1.050 & 0.1309 & 0.969 \\
\hline OC D & 50.3 & 4.675 & 0.986 & 0.5900 & 1.046 & 0.1326 & 0.967 \\
\hline \multirow[t]{3}{*}{$O C D$} & 26.2 & 4.702 & 0.987 & 0.5970 & 1.040 & 0.1344 & 0.960 \\
\hline & & & $\rightarrow-m$ & & 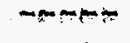 & & artar \\
\hline & $\begin{array}{l}\text { ne Aver } \\
\text { dard De }\end{array}$ & & $\begin{array}{l}0.998 \\
0.011\end{array}$ & & $\begin{array}{l}1.043 \\
0.015\end{array}$ & & $\begin{array}{l}0.949 \\
0.023\end{array}$ \\
\hline
\end{tabular}

${ }^{a}$ Cell factor not measured for ${ }^{238} \mathrm{U}$ capture in the axial blanket. 
TABLE 4.13 ZPPR-18B: Axial Reaction Rate Distributions in Matrix 149-75 (outer core)

\begin{tabular}{|c|c|c|c|c|c|c|c|}
\hline \multirow[b]{2}{*}{ Zone } & \multirow[b]{2}{*}{$\underline{z(m m)}$} & \multicolumn{2}{|c|}{$235 \mathrm{U}(\mathrm{n}, \mathrm{f})$} & \multicolumn{2}{|c|}{${ }^{238} \mathrm{U}(\mathrm{n}, \gamma)^{\mathrm{a}}$} & \multicolumn{2}{|c|}{${ }^{2}{ }^{38} \mathrm{U}(n, f)$} \\
\hline & & Exp. & $C / E$ & Exp. & $C / E$ & Exp. & $C / E$ \\
\hline$O C D$ & 26.2 & 4.735 & 0.993 & 0.6147 & 1.024 & 0.1379 & 0.949 \\
\hline$O C D$ & 50.3 & 4.728 & 1.001 & 0.6143 & 1.031 & 0.1371 & 0.960 \\
\hline OC D & 77.0 & 4.795 & 0.990 & 0.6097 & 1.042 & 0.1392 & 0.949 \\
\hline$O C D$ & 127.8 & 4.686 & 0.987 & 0.6019 & 1.028 & 0.1367 & 0.940 \\
\hline OC D & 178.6 & 4.565 & 0.986 & 0.5853 & 1.029 & 0.1303 & 0.958 \\
\hline OC D & 229.4 & 4.395 & 0.986 & 0.5603 & 1.035 & 0.1250 & 0.958 \\
\hline$O C D$ & 280.2 & 4.107 & 0.984 & 0.5271 & 1.026 & 0.1187 & 0.931 \\
\hline$O C D$ & 331.0 & 3.770 & 0.994 & 0.4887 & 1.027 & 0.1070 & 0.947 \\
\hline$O C D$ & 381.8 & 3.463 & 0.989 & 0.4459 & 1.029 & 0.0957 & 0.933 \\
\hline$O C D$ & 432.6 & 3.075 & 0.996 & 0.3900 & 1.053 & 0.0806 & 0.917 \\
\hline \multirow[t]{3}{*}{$O C D$} & 483.4 & 2.704 & 1.008 & 0.3440 & 1.059 & 0.0638 & 0.875 \\
\hline & & & 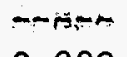 & & 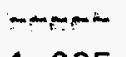 & & matima \\
\hline & \multicolumn{2}{|c|}{$\begin{array}{l}\text { Zone Average } C / E \\
\text { tandard Deviation }\end{array}$} & $\begin{array}{l}0.992 \\
0.007\end{array}$ & & $\begin{array}{l}1.035 \\
0.012\end{array}$ & & $\begin{array}{l}0.938 \\
0.025\end{array}$ \\
\hline$A B$ & 534.2 & 2.534 & 0.994 & -6 & $\rightarrow$ & 0.0295 & 0.977 \\
\hline$A B$ & 585.0 & 2.279 & 0.989 & -4 & $-\infty$ & 0.0177 & 1.043 \\
\hline$A B$ & 635.8 & 2.006 & 1.007 & -6 & m & 0.0131 & 0.816 \\
\hline$A B$ & 686.6 & 1.735 & 1.055 & $\rightarrow$ & $-\infty$ & 0.0080 & 0.884 \\
\hline \multirow[t]{3}{*}{$A B$} & 737.4 & 1.584 & 1.056 & $\rightarrow$ & i & -4 & -- \\
\hline & & & $\rightarrow m-m$ & & & & חיד, \\
\hline & $\begin{array}{l}\text { he Aver } \\
\text { dard De }\end{array}$ & & $\begin{array}{l}1.020 \\
0.033\end{array}$ & & & & $\begin{array}{l}0.930 \\
0.100\end{array}$ \\
\hline
\end{tabular}

${ }^{a} \mathrm{Cell}$ factor not measured for ${ }^{23} \mathrm{U}$ capture in the axial blanket. 
TABLE 4.14 ZPPR-18B: Axial $235 \mathrm{U}$ Fission Distributions in Matrix 246-57 and 146-57

\begin{tabular}{|c|c|c|c|c|c|c|c|}
\hline \multirow[b]{2}{*}{ Zone } & \multicolumn{3}{|c|}{ Matrix 246-57 } & & & \multicolumn{2}{|c|}{ Matrix $146-57^{\mathrm{b}}$} \\
\hline & $\underline{z}(\mathrm{~mm})$ & Exp. & $C / E$ & Zone & $z(\mathrm{~mm})$ & Exp. & $C / E$ \\
\hline$A B$ & 737.4 & 1.371 & 1.013 & $\mathrm{AB}$ & 737.4 & 3.655 & 1.040 \\
\hline$A B$ & 686.6 & 1.556 & 1.016 & $A B$ & 686.6 & 3.990 & 1.041 \\
\hline$A B$ & 635.8 & 1.818 & 1.001 & $A B$ & 635.8 & 4.420 & 1.027 \\
\hline$A B$ & 585.0 & 2.139 & 1.000 & $A B$ & 585.0 & 4.942 & 1.007 \\
\hline \multirow[t]{2}{*}{$A B$} & 534.2 & 2.521 & 0.993 & $A B$ & 534.2 & 5.400 & $\begin{array}{l}1.009 \\
m-\infty \sim m\end{array}$ \\
\hline & $\begin{array}{l}\text { one Ave } \\
\text { idard D }\end{array}$ & $\begin{array}{l}C / E \\
\text { tion }\end{array}$ & $\begin{array}{l}1.005 \\
0.010\end{array}$ & \multicolumn{3}{|c|}{$\begin{array}{r}\text { Zone Average } C / E \\
\text { Standard Deviation }\end{array}$} & $\begin{array}{l}1.025 \\
0.016\end{array}$ \\
\hline IC SM & 483.4 & 2.885 & 0.995 & IC SM & 483.4 & 5.551 & 1.026 \\
\hline IC SM & 432.6 & 3.362 & 0.990 & IC SM & 432.6 & 6.106 & 1.018 \\
\hline IC SM & 381.8 & 3.821 & 0.995 & IC $S M$ & 381.8 & 6.674 & 1.014 \\
\hline IC SM & 331.0 & 4.254 & 1.002 & IC SM & 331.0 & 7.227 & 1.003 \\
\hline IC SM & 280.2 & 4.742 & 0.994 & IC SM & 280.2 & 7.676 & 1.001 \\
\hline IC SM & 229.4 & 5.181 & 0.997 & IC SM & 229.4 & 8.036 & 1.010 \\
\hline IC SM & 178.6 & 5.595 & 1.002 & IC SM & 178.6 & 8.253 & 0.996 \\
\hline IC SM & 127.8 & 5.970 & 1.011 & IC SM & 127.8 & 8.303 & 0.990 \\
\hline IC SM & 77.0 & 6.461 & 1.001 & IC SM & 77.0 & 8.160 & 1.007 \\
\hline IC SM & 50.3 & 6.668 & 1.015 & IC SM & 50.3 & 7.984 & 0.997 \\
\hline \multirow[t]{4}{*}{ IC SM } & 26.2 & 7.052 & 1.000 & IC SM & 26.2 & 7.695 & 0.997 \\
\hline & & & $m-m-m-$ & & & & \\
\hline & one Ave & $\mathrm{C} / \mathrm{E}$ & 1.000 & & one Ave & $C / E$ & 0.995 \\
\hline & dard D & tion & 0.007 & & ndard $D$ & tion & 0.011 \\
\hline
\end{tabular}

${ }^{a}$ Adjacent to control rod.

b Adjacent to CRP. 
TABLE 4.15 Summary of C/E Results for ${ }^{25} \mathrm{U}$ Fission in $Z P P R-18 B$

\begin{tabular}{|c|c|c|c|c|}
\hline Radial Distribution ${ }^{a}$ & Zone & $\begin{array}{c}\text { Number of } \\
\text { Folls } \\
\end{array}$ & $\begin{array}{r}\text { Mean } \\
\mathrm{C} / \mathrm{E} \\
\end{array}$ & $\begin{array}{l}\text { Standard } \\
\text { Deviation }\end{array}$ \\
\hline \multirow[t]{2}{*}{$z=+28 \mathrm{~cm}$} & Inner Core & 45 & 0.993 & 0.008 \\
\hline & Outer Core & 7 & 0.981 & 0.006 \\
\hline \multirow[t]{2}{*}{$z=+5 \mathrm{~cm}$} & Inner Core & 46 & 1.002 & 0.007 \\
\hline & Outer Core & 11 & 0.992 & 0.009 \\
\hline \multirow[t]{2}{*}{$z=-5 \mathrm{~cm}$} & Inner Core & 60 & 1.006 & 0.010 \\
\hline & outer Core & 11 & 0.998 & 0.013 \\
\hline \multirow[t]{2}{*}{$z=-28 \mathrm{~cm}$} & Inner Core & 61 & 1.005 & 0.011 \\
\hline & Outer Core & 11 & 1.000 & 0.013 \\
\hline
\end{tabular}

${ }^{a}$ Results at the $x$-axis, $15^{\circ}$ and $30^{\circ}$ combined. Results along the yraxis excluded. 
TABLE 4.16 ZPPR-18B: Reaction Rate Ratios along the $x$ raxis at $z=-5.16 \mathrm{~cm}$

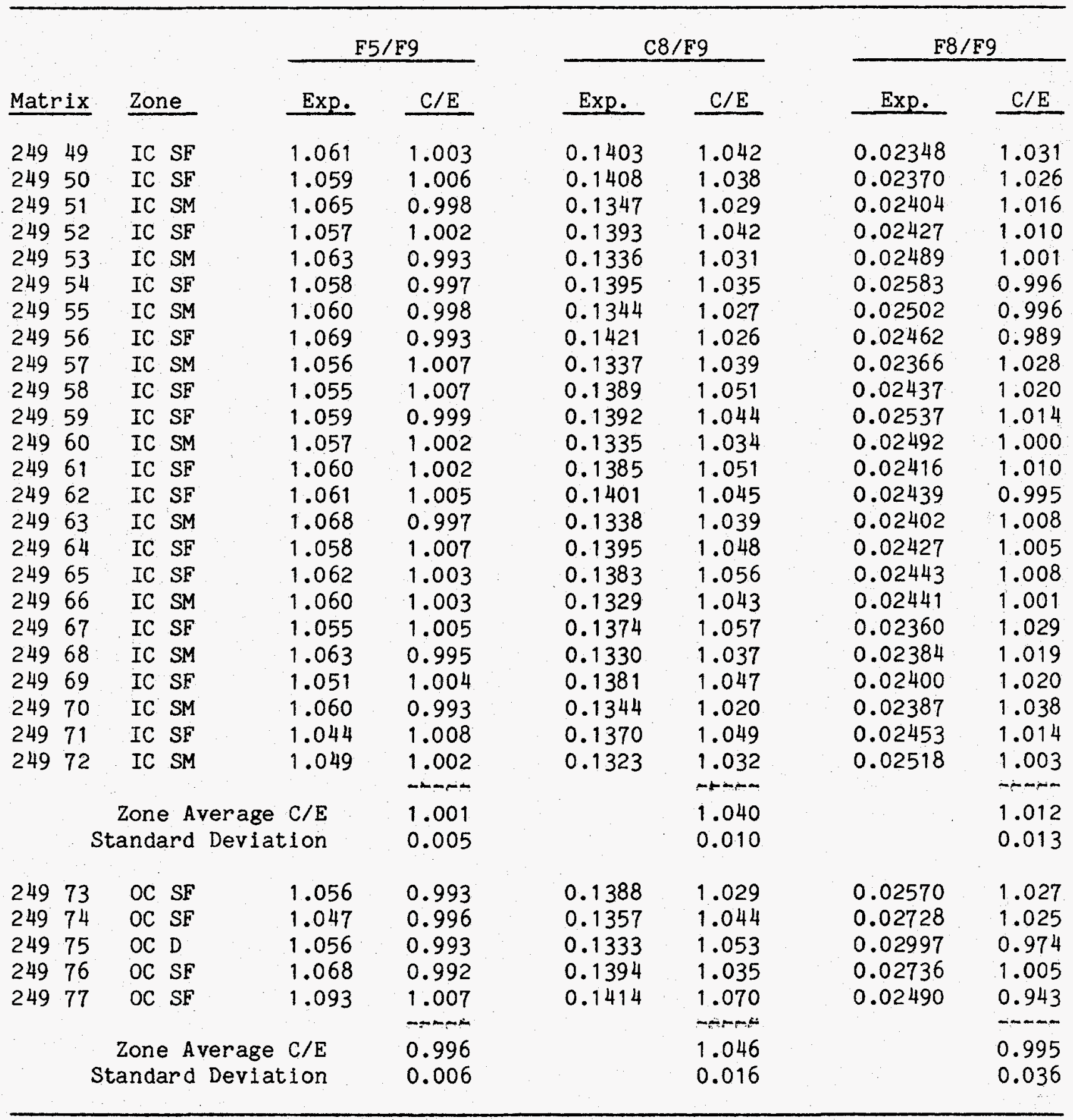


TABLE 4.17 ZPPR-18B: Reaction Rate Ratios at $15^{\circ}$ to the $x^{-a x i s}$ at $z=-5.16 \mathrm{~cm}$

\begin{tabular}{|c|c|c|c|c|c|c|c|}
\hline \multirow[b]{2}{*}{ Matrix } & \multirow[b]{2}{*}{ Zone } & \multicolumn{2}{|c|}{$\mathrm{F} 5 / \mathrm{F} 9$} & \multicolumn{2}{|c|}{$\mathrm{C} 8 / \mathrm{Fg}$} & \multicolumn{2}{|c|}{$\mathrm{F} 8 / \mathrm{F} 9$} \\
\hline & & Exp. & $\mathrm{C} / \mathrm{E}$ & Exp. & $C / E$ & Exp. & $C / E$ \\
\hline 24850 & IC SF & 1.044 & 1.013 & 0.1403 & 1.039 & 0.02402 & 1.013 \\
\hline 24852 & IC SM & 1.055 & 1.002 & 0.1334 & 1.034 & 0.02431 & 1.011 \\
\hline 24853 & IC $S F$ & 1.047 & 1.005 & 0.1375 & 1.053 & 0.02486 & 1.010 \\
\hline 24754 & IC SF & 1.054 & 0.990 & 0.1388 & 1.041 & 0.02503 & 0.995 \\
\hline 24756 & IC SF & 1.037 & 0.999 & 0.1397 & 1.042 & 0.02418 & 0.989 \\
\hline 24757 & IC SF & 1.027 & 1.008 & 0.1370 & 1.064 & 0.02396 & 0.996 \\
\hline 24657 & IC $S M$ & 0.977 & 1.025 & 0.1323 & 1.042 & 0.02303 & 0.998 \\
\hline $246 \quad 58$ & IC SF & 1.035 & 0.999 & 0.1377 & 1.055 & 0.02388 & 0.988 \\
\hline $246 \quad 59$ & IC SM & 1.048 & 1.000 & 0.1335 & 1.033 & 0.02403 & 0.999 \\
\hline 24660 & IC SF & 1.053 & 1.000 & 0.1379 & 1.053 & 0.02381 & 1.013 \\
\hline 24561 & IC $S F$ & 1.038 & 1.019 & 0.1354 & 1.074 & 0.02409 & 1.024 \\
\hline 24562 & IC SM & 1.057 & 1.001 & 0.1343 & 1.029 & 0.02441 & 0.992 \\
\hline 24463 & IC SM & 1.062 & 1.001 & 0.1333 & 1.039 & 0.02385 & 1.000 \\
\hline 244.64 & IC SF & 1.063 & 0.999 & 0.1388 & 1.051 & 0.02391 & 0.995 \\
\hline 244.65 & IC SM & 1.058 & 1.001 & 0.1324 & 1.043 & 0.02326 & 1.031 \\
\hline 24466 & IC SF & 1.055 & 1.001 & 0.1392 & 1.044 & 0.02397 & 1.015 \\
\hline 24367 & IC SF & 1.043 & 0.987 & 0.1398 & 1.038 & 0.02477 & 0.985 \\
\hline 24368 & IC SF & 1.042 & 1.005 & 0.1387 & 1.043 & 0.02429 & 1.019 \\
\hline 24369 & IC SM & 1.042 & 1.003 & 0.1330 & 1.033 & 0.02431 & 1.000 \\
\hline 24370 & IC SF & 1.017 & 1.011 & 0.1398 & 1.029 & 0.02433 & 0.977 \\
\hline \multirow[t]{4}{*}{24270} & $\begin{array}{l}\text { IC SM } \\
\text { CR }\end{array}$ & 1.044 & 1.004 & 0.1359 & 1.017 & 0.02478 & 0.964 \\
\hline & & & Anth & & بctaram & & $m-m-a$ \\
\hline & \multirow{2}{*}{\multicolumn{2}{|c|}{$\begin{array}{l}\text { Zone Average C/E } \\
\text { Standard Deviation }\end{array}$}} & 1.003 & & 1.043 & & 1.001 \\
\hline & & & 0.008 & & 0.013 & & 0.016 \\
\hline 24273 & OC SF & 1.024 & 1.014 & 0.1397 & 1.028 & 0.02632 & 0.977 \\
\hline 24173 & OC SF & 1.063 & 0.997 & 0.1409 & 1.025 & 0.02615 & 1.016 \\
\hline 24174 & OC SF & 1.050 & 0.996 & 0.1384 & 1.032 & 0.02750 & 0.998 \\
\hline 24175 & $O C D$ & 1.042 & 1.006 & 0.1357 & 1.040 & 0.03035 & 0.941 \\
\hline 24176 & $O C S F$ & 1.047 & 0.999 & 0.1371 & 1.047 & 0.02758 & 1.004 \\
\hline \multirow[t]{4}{*}{24077} & $\mathrm{OC} \mathrm{SF}$ & 1.086 & 0.988 & 0.1426 & 1.042 & 0.02609 & 0.946 \\
\hline & & & 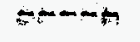 & & $-m+n+c$ & & \\
\hline & Zone Av & $C / E$ & 1.000 & & 1.036 & & 0.980 \\
\hline & tandard & tion & 0.009 & & 0.009 & & 0.031 \\
\hline
\end{tabular}


TABLE 4.18 ZPPR-18B: Reaction Rate Ratios in Matrix 249-49 and 149-49 (core center)

\begin{tabular}{|c|c|c|c|c|c|c|c|c|}
\hline \multirow[b]{2}{*}{ Matrix } & \multirow[b]{2}{*}{ Zone } & \multirow[b]{2}{*}{$\mathrm{z}(\mathrm{mm})$} & \multicolumn{2}{|c|}{ F5/F9 } & \multicolumn{2}{|c|}{$\mathrm{C} 8 / \mathrm{F} 9$} & \multicolumn{2}{|c|}{$\mathrm{F} 8 / \mathrm{F} 9$} \\
\hline & & & Exp. & $C / E$ & Exp. & $\mathrm{C} / \mathrm{E}$ & Exp. & $C / E$ \\
\hline 24949 & IC SF & 483.4 & 1.102 & 1.037 & 0.1453 & 1.077 & 0.02028 & 0.947 \\
\hline 24949 & IC SF & 432.6 & 1.089 & 1.017 & 0.1438 & 1.057 & 0.02149 & 1.012 \\
\hline 24949 & IC SF & 380.5 & 1.077 & 1.009 & 0.1439 & 1.038 & 0.02300 & 1.006 \\
\hline 24949 & IC SF & 356.4 & 1.087 & 0.993 & 0.1440 & 1.031 & 0.02391 & 0.987 \\
\hline $249 \quad 49$ & IC SF & 280.2 & 1.072 & 1.000 & 0.1414 & 1.042 & 0.02352 & 1.017 \\
\hline $249 \quad 49$ & IC SF & 229.4 & 1.065 & 1.003 & 0.1410 & 1.041 & 0.02404 & 1.003 \\
\hline $249 \quad 49$ & IC SF & 178.6 & 1.061 & 1.005 & 0.1418 & 1.033 & 0.02353 & 1.027 \\
\hline 24949 & IC SF & 127.8 & 1.066 & 1.000 & 0.1412 & 1.037 & 0.02366 & 1.023 \\
\hline 24949 & IC SF & 77.0 & 1.073 & 0.993 & 0.1412 & 1.036 & 0.02429 & 0.997 \\
\hline 24949 & IC SF & 50.3 & 1.061 & 1.003 & 0.1403 & 1.042 & 0.02348 & 1.031 \\
\hline $249 \quad 49$ & IC SF & 26.2 & 1.052 & 1.013 & 0.1388 & 1.054 & 0.02398 & 1.011 \\
\hline 14949 & IC SF & 26.2 & 1.055 & 1.010 & 0.1385 & 1.056 & 0.02346 & 1.033 \\
\hline $149 \quad 49$ & IC $\mathrm{SF}$ & 50.3 & 1.073 & 0.992 & 0.1413 & 1.035 & 0.02408 & 1.006 \\
\hline 14949 & IC SF & 77.0 & 1.065 & 1.001 & 0.1413 & 1.036 & 0.02417 & 1.003 \\
\hline $149 \quad 49$ & IC SF & 127.8 & 1.062 & 1.004 & 0.1407 & 1.042 & 0.02383 & 1.017 \\
\hline $149 \quad 49$ & IC SF & 178.6 & 1.065 & 1.003 & 0.1408 & 1.041 & 0.02369 & 1.021 \\
\hline 14949 & IC SF & 229.4 & 1.059 & 1.010 & 0.1398 & 1.050 & 0.02398 & 1.006 \\
\hline 14949 & IC SF & 280.2 & 1.073 & 1.001 & 0.1429 & 1.033 & 0.02397 & 0.998 \\
\hline $149 \quad 49$ & IC SF & 356.4 & 1.079 & 1.003 & 0.1435 & 1.035 & 0.02334 & 1.008 \\
\hline 14949 & IC SF & 380.5 & 1.094 & 0.996 & 0.1441 & 1.039 & 0.02323 & 0.991 \\
\hline $149 \quad 49$ & IC SF & 432.6 & 1.093 & 1.018 & 0.1441 & 1.059 & 0.02259 & 0.955 \\
\hline \multirow[t]{3}{*}{14949} & IC SF & 483.4 & 1.111 & 1.035 & 0.1453 & 1.082 & 0.02055 & 0.922 \\
\hline & & & & $\cdots+\cdots$ & & 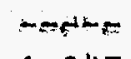 & & 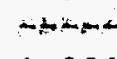 \\
\hline & \multicolumn{2}{|c|}{$\begin{array}{l}\text { Zone Average } C / E \\
\text { Standard Deviation }\end{array}$} & & $\begin{array}{l}1.007 \\
0.012\end{array}$ & & $\begin{array}{l}1.045 \\
0.014\end{array}$ & & 1.001 \\
\hline
\end{tabular}


TABLE 4.19 ZPPR-18B: Summary of Reaction Rate Ratio Analysis

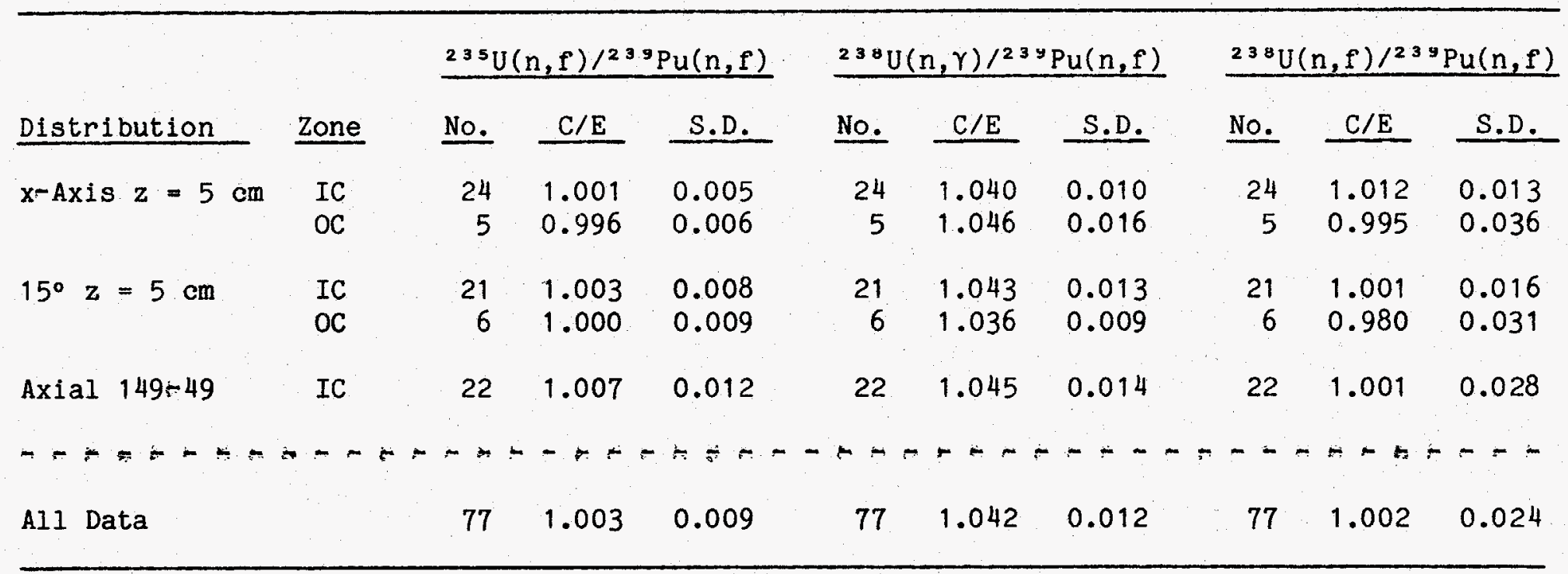




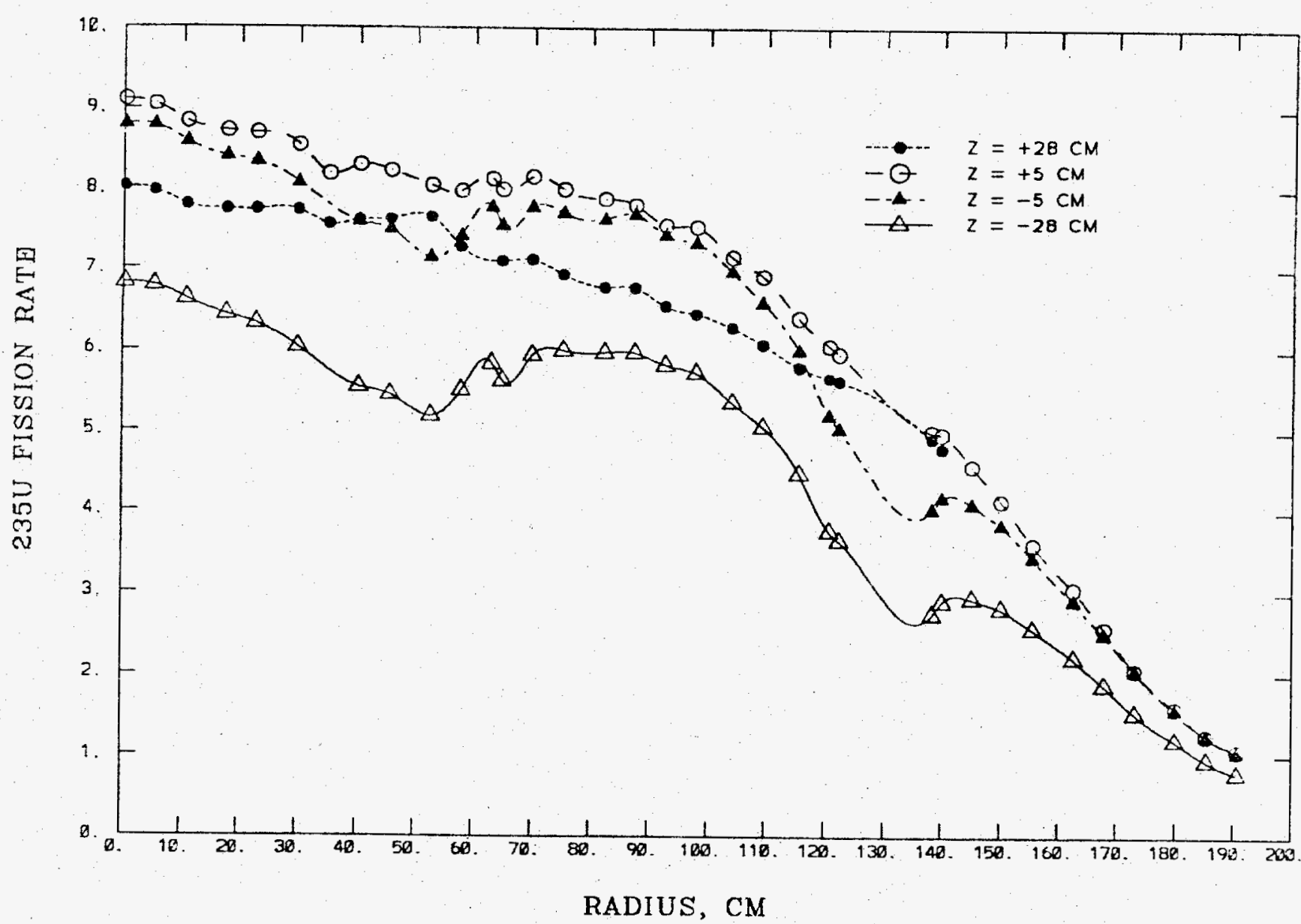

Fig. 4.1 Measured ${ }^{23} \mathrm{U}$ Radial Fission Distributions at Four Axial Heights at $15^{\circ}$ to the X-axis of ZPPR-18B 


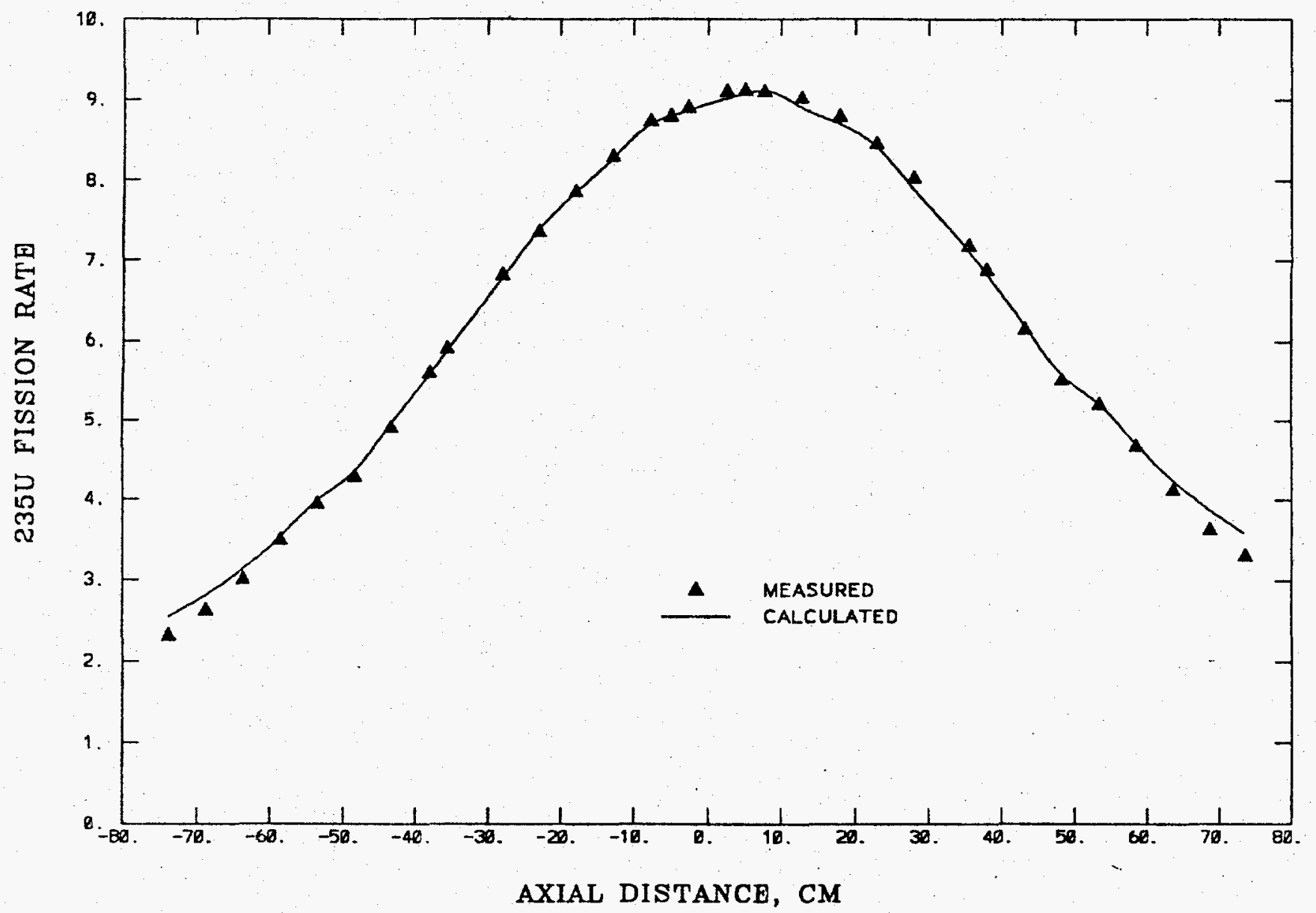

F18. 4.2 Measured and Calculated Axial Distributions for ${ }^{23} \mathrm{U}$ Fission Near the Center of ZPPR-18B 


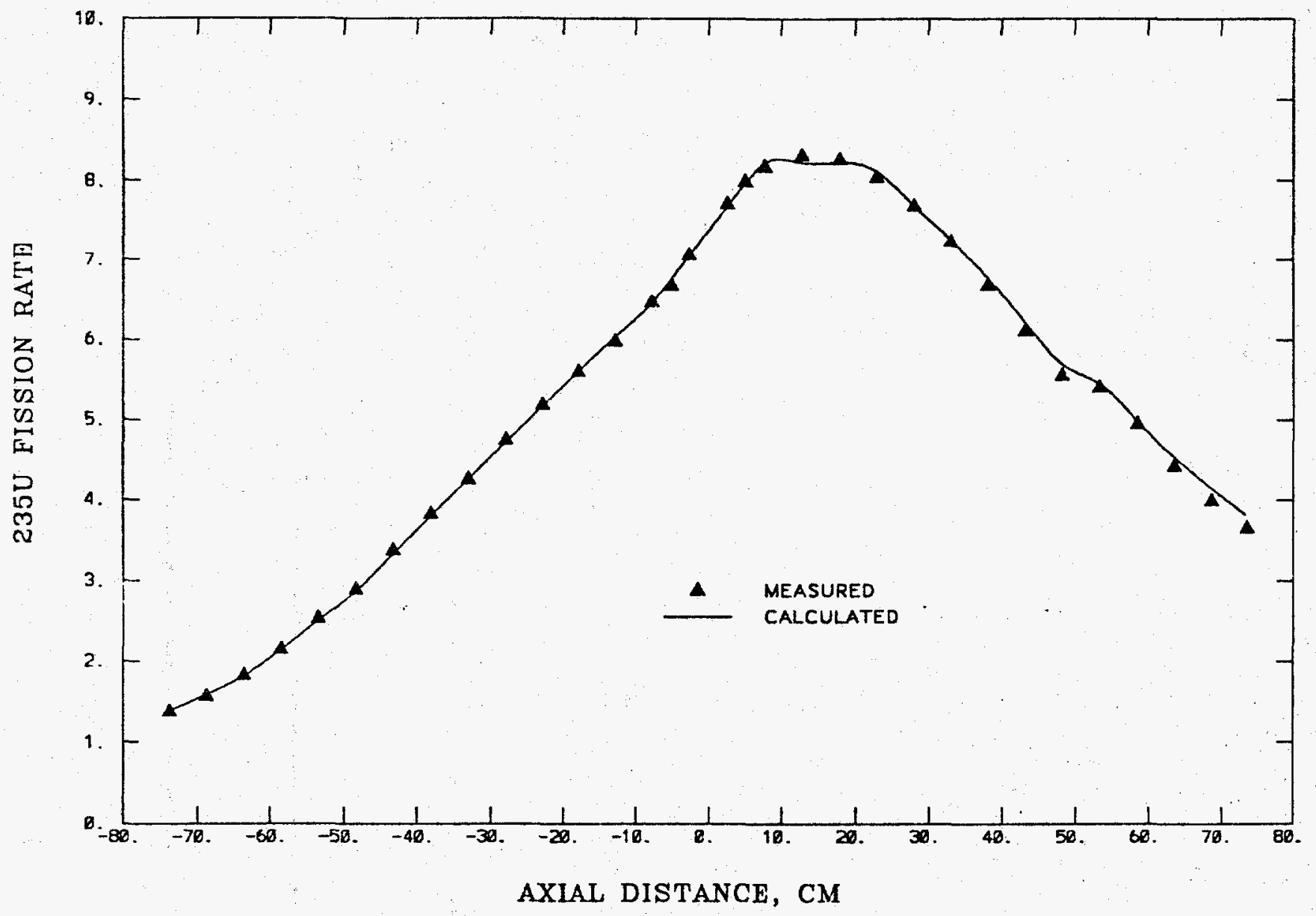

Fig. 4.3 Measured and Calculated Axial Distributions for ${ }^{23} \mathrm{U}$ Fission Next to an Inner Ring Control rod in $\mathrm{ZPPR}-18 \mathrm{~B}$ 


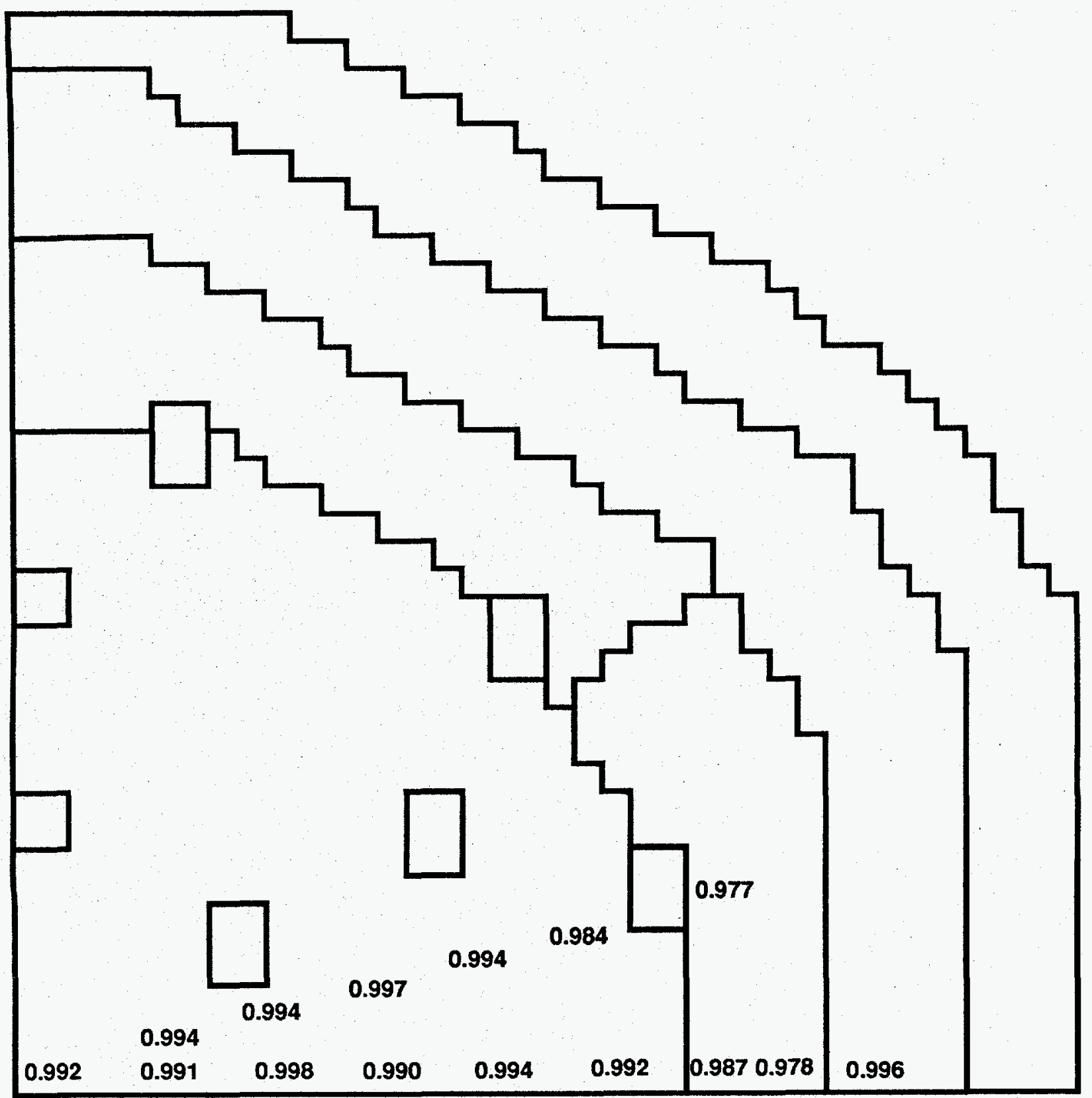

Fig. 4.4 Summary of C/E Results for $235 \mathrm{U}$ Fission in ZPPR $18 \mathrm{~B}$ at $28 \mathrm{~cm}$ Above the Midplane (CRP half) 


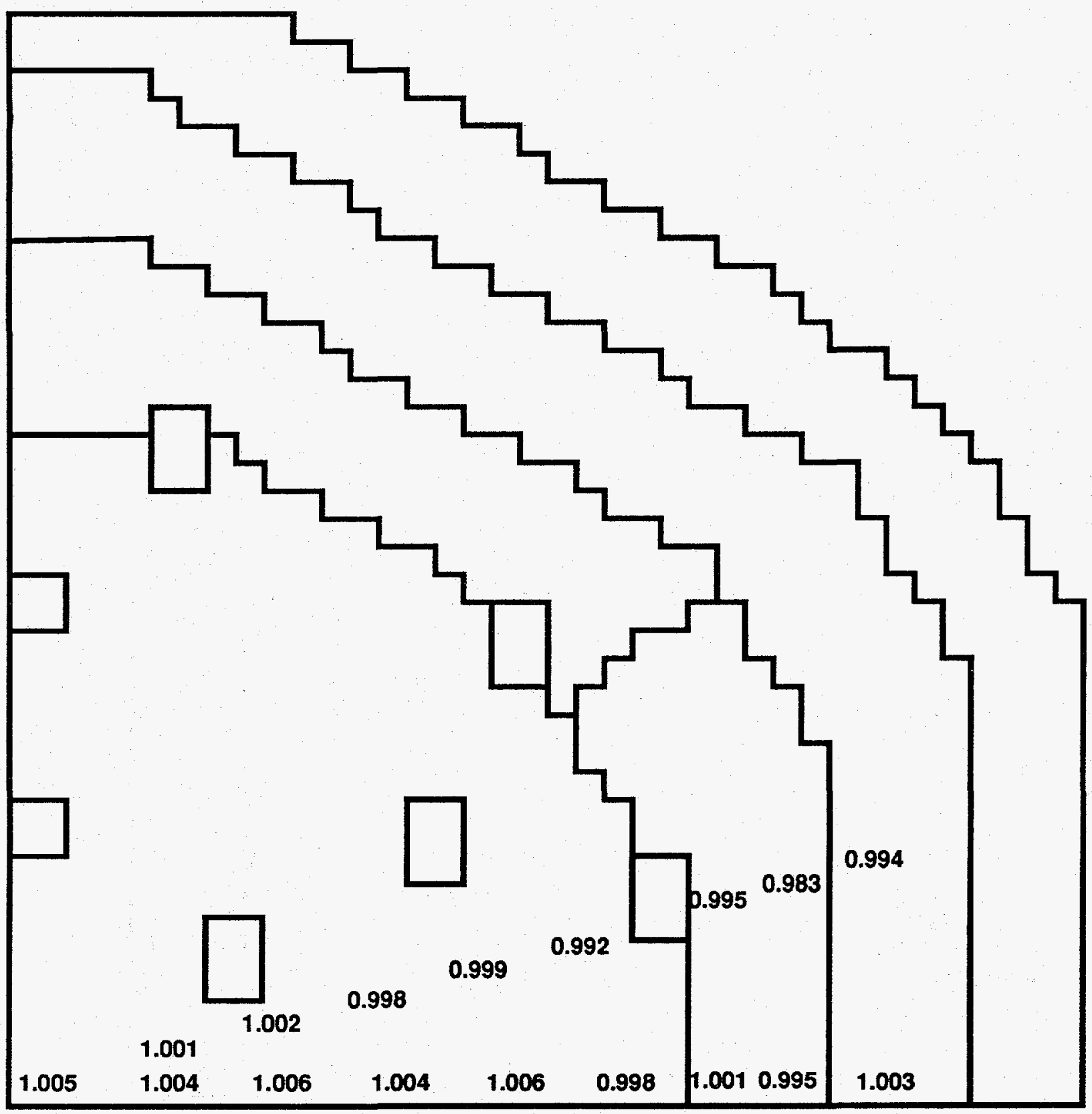

Fig. 4.5 Summary of C/E Results for $235 \mathrm{U}$ Fission in ZPPR-18B at $5 \mathrm{~cm}$ Above the Midplane (CRP half) 


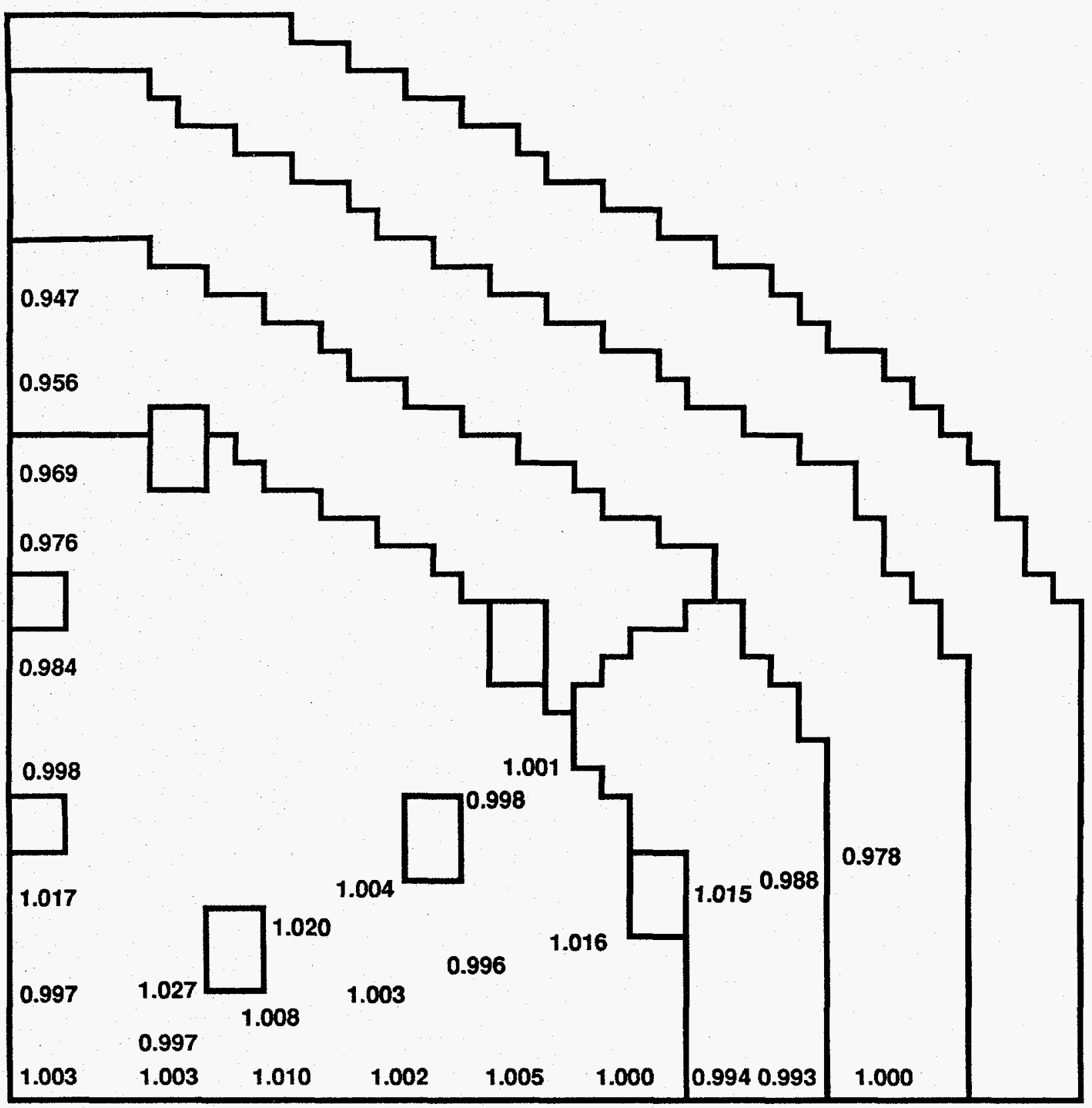

Fig. 4.6 Summary of C/E Results for ${ }^{235} \mathrm{U}$. Fission in ZPPR-18B at $5 \mathrm{~cm}$ Below the Midplane (CR half) 


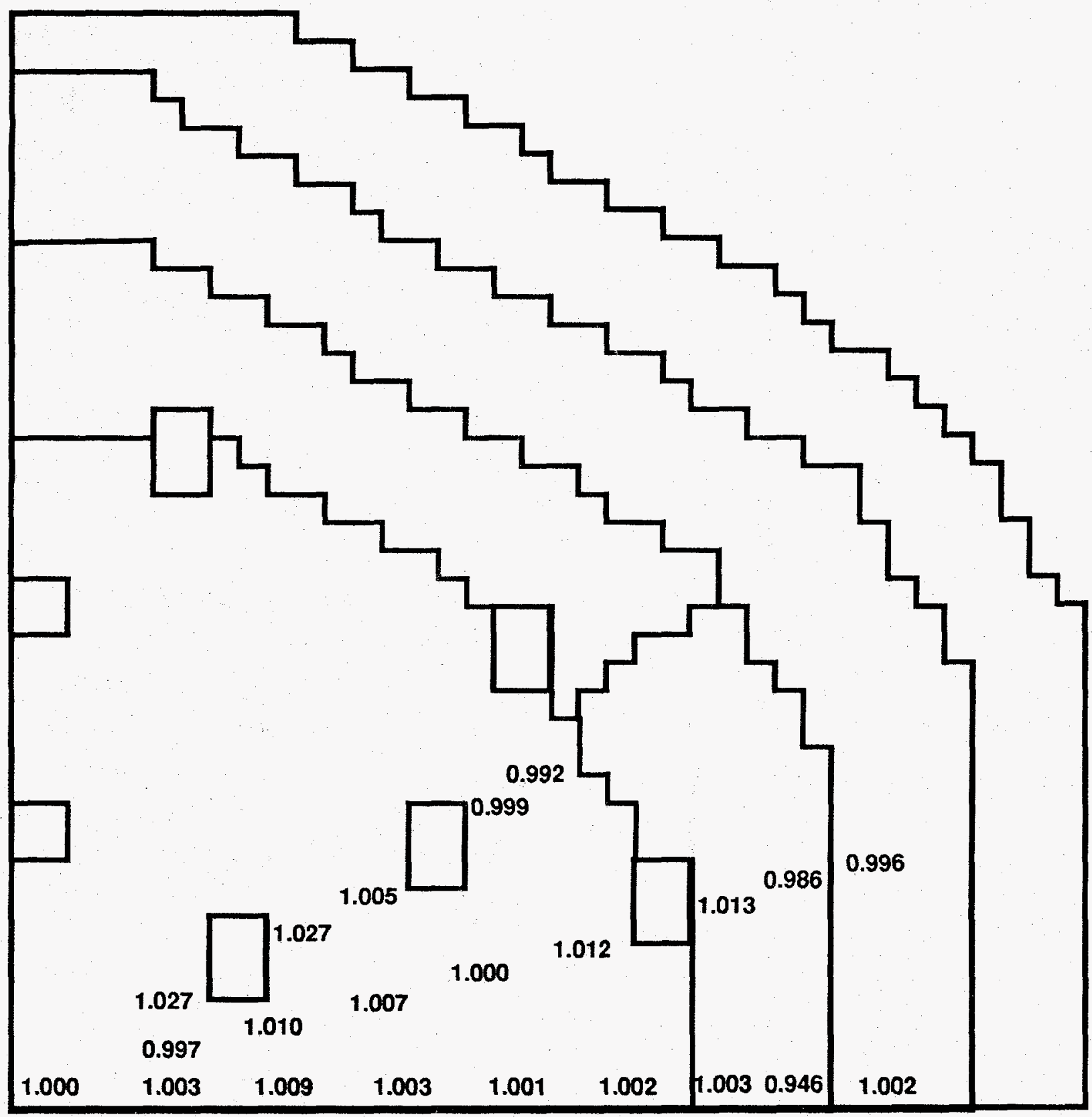

Fig. 4.7 Summary of $\mathrm{C} / \mathrm{E}$ Results for ${ }^{235} \mathrm{U}$ Fission in $\mathrm{ZPPR}-18 \mathrm{~B}$ at $28 \mathrm{~cm}$ Below the Midplane (CR half) 
5. BASIC DATA FOR REACTION RATE DISTRIBUTIONS IN ZPPR-18A AND ZPPR-18B (D. W. Maddison and J. M. Gasidlo)

\subsection{Introduction}

Reaction rate maps are measured by first choosing a location in a ceil to place the irradiation foil. These "mapping" foils are always placed in the same location in a drawer for a specific type of cell. Then, a pattern of drawers is chosen to provide the reactor distribution desired, such as a radial map or an axial distribution.

\subsection{Discussion of Foil Locations}

The foils were first placed in stainless steel foll holders which were then loaded into the drawers between the vertical columns of materials. For drawers with one fuel column the foil was placed adjacent to the fuel on the side closer to the core center. For drawers with two fuel columns, the foll was placed adjacent to the fuel plate closer to the core center on the side of the plate closer to the drawer center. The drawer column for the mapping foils in any matrix position in the reactor are given below.

Matrix Columns $\leq 48$

Reactor Half 1

Core

$\mathrm{AB}$

$\mathrm{RB}$

$\mathrm{RR} / \mathrm{AR}$

$\begin{array}{lll}\text { SF,SM } & & \text { D } \\ & & \text { JK } \\ \text { IJ } & & \text { IJ } \\ \text { IJ } & & \\ & \text { IJ } & \\ & \text { TC } & \end{array}$

TC $\underline{\text { Matrix Column } \geq 49}$

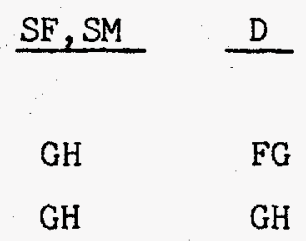

GH

TC

\footnotetext{
*ZPPR drawers are divided into sixteen $3.175 \mathrm{~mm}(1 / 8 \mathrm{in.})$ wide vertical columns designed by letter's A through P. The letter A denotes the column at the left as one faces the drawer. The letters FG, for example, indicate the foil is between columns $F$ and $G$, and is centered $19.050 \mathrm{~mm}(0.750$ in.) from the left side of the drawer.
} 
Matrix Columns $\leq 48$

Reactor Half 2

Core

$\mathrm{AB}$

$\mathrm{RB}$

$\mathrm{RR} / \mathrm{AR}$

$$
\mathrm{SF}, \mathrm{SM}
$$

GH

$\mathrm{GH}$

$\mathrm{GH}$

TC
Matrix Column $\geq 49$

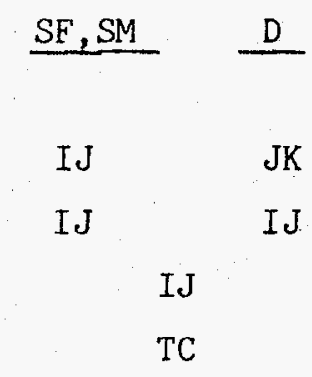

Where SF is a drawer with one fuel column that has iron oxide plates on both sides of the fuel plate, SM is a drawer with one fuel column that has depleted uranium metal plates on both sides of the fuel plate, $D$ is a drawer with two fuel columns, core is the fueled region, $A B$ is the axial blanket in a fueled drawer, $R B$ is the radial blanket and $A R / R R$ is the axial or radial reflector. There are some ${ }^{235} \mathrm{U}$ foils in the axial and radial reflectors which are given a drawer location of "TC". For this location, the foil holder was placed horizontally across the top of the drawer. The foil was centered above drawer columns $\mathrm{HI}$ and the foil was centered $25.654 \mathrm{~mm}$ above the mid-height of the drawer.

The basic data for the radial maps and axial distributions in ZPPR-18A are given in Tables 5.1 - 5.3. The basic data for $Z P P R-18 B$ were measured in two irradiations. There were 10 common locations for ${ }^{235} \mathrm{U}$ folls in the two irradiations for comparing the power histories. The welghted average fission ratio of the common foils was 1.0008 with one standard deviation of 0.0020 . Since this was close to unity, the two data sets were merged using the average of the two foils for the common locations. The basic data for the merged data sets are 8 iven in Tables $5.4-5.8$.

In both $18 \mathrm{~A}$ and $18 \mathrm{~B}$, all foils were centered on the listed axial positions as measured from the reactor midplane. The ${ }^{235} \mathrm{U}$ folls were centered $13.8 \mathrm{~mm}$ above, the ${ }^{238} \mathrm{U}$ foils were centered on, and the ${ }^{239} \mathrm{Pu}$ foils were centered $13.8 \mathrm{~mm}$ below the mid-height of the drawer. 
The core configurations and operating rod parameters for $18 \mathrm{~A}$ are given in ANL-ZPR-489, Section 8, and for $18 \mathrm{~B}$ in Section 4 of this report.

\subsection{Discussion of Foll Data and Uncertainties}

In all of the tables, the data are given in units of $10^{-18}$ fissions or captures per sec per atom in the irradiated foll at an estimated reactor power of one watt. The second number assoclated with each reaction rate is a one standard deviation estimate of the random uncertainty. The uncertainties given in the tables are solely due to counting statistios and data reduction. They include the effects of peak integration, interference peak corrections, corrections for other reactions in the foils, irradiation history and foil positioning in the counter system. Other uncertalnties due to cell-averaging factors are discussed in Section 6. Other uncertainties such as those due to detector calibrations are not included. The uncertainties are discussed in greater detail by Brumbach and Gasidlo. ${ }^{1}$

REFERENCE

1. "In-Cell Reaction Rate Distributions and Cell-Average Reaction Rates in Fast Critical Assemblies," ANL-85-44, August 1985. 
TABLE 5.1 Basic Data for Radial Distributions of ${ }^{23} \mathrm{Pu}(\mathrm{n}, \mathrm{f})$ in $\mathrm{ZPPR}-18 \mathrm{~A}$

\begin{tabular}{|c|c|c|c|c|c|c|}
\hline Matrix & $\underline{L o c}{ }^{a}$ & $2{ }^{39} \mathrm{Pu}(\mathrm{n}, \mathrm{f})^{\mathrm{b}}$ & Matrix & Loc & \multicolumn{2}{|c|}{$2{ }^{39} \mathrm{Pu}(\mathrm{n}, \mathrm{f})^{\mathrm{b}}$} \\
\hline$x^{2}-a x i s$ & data at & $z=51.6 \mathrm{~mm}$ & \multicolumn{4}{|c|}{$15^{\circ}$ data at $\mathrm{z}=51.6 \mathrm{~mm}$} \\
\hline 14950 & $\mathrm{GH}$ & $8.790 \quad 0.043$ & 14850 & $\mathrm{GH}$ & 8.738 & 0.042 \\
\hline 14951 & GH & $8.474 \cdot 0.044$ & 14852 & GH & 8.515 & 0.039 \\
\hline 14952 & $\mathrm{GH}$ & 8.6510 .040 & 14853 & GH & 8.608 & 0.040 \\
\hline 14953 & $\mathrm{GH}$ & $\begin{array}{lll}8.473 & 0.042\end{array}$ & 14754 & $\mathrm{GH}$ & 8.386 & 0.041 \\
\hline 14954 & GH & $8.472 \quad 0.040$ & 14755 & $\mathrm{GH}$ & 8.261 & 0.041 \\
\hline 14955 & GH & 8.2970 .042 & 14756 & GH & 8.360 & 0.041 \\
\hline 14956 & $\mathrm{GH}$ & 8.3690 .043 & 14757 & $\mathrm{GH}$ & 8.344 & 0.039 \\
\hline 14957 & GH & 8.2320 .040 & 14657 & $\mathrm{GH}^{*}$ & 8.253 & 0.039 \\
\hline 14958 & $\mathrm{GH}$ & $8.320 \quad 0.039$ & 14658 & $\mathrm{GH}$ & 8.269 & 0.040 \\
\hline 14959 & $\mathrm{GH}$ & $8.100 \quad 0.040$ & 14659 & GH & 7.938 & 0.037 \\
\hline 14960 & GH & 7.8550 .038 & 14660 & $\mathrm{GH}$ & 7.927 & 0.039 \\
\hline 14961 & GH & $\begin{array}{lll}7.884 & 0.043\end{array}$ & 14560 & $\mathrm{GH}$ & 7.857 & 0.038 \\
\hline 14962 & GH & 7.8320 .041 & 14561 & $\mathrm{GH}$ & 7.836 & 0.038 \\
\hline 14963 & GH & $7.607 \quad 0.036$ & 14562 & $\mathrm{GH}$ & 7.512 & 0.038 \\
\hline 14964 & $\mathrm{GH}$ & $7.609 \quad 0.035$ & 14463 & $\mathrm{GH}$ & 7.302 & 0.035 \\
\hline 14965 & $\mathrm{GH}$ & $\begin{array}{lll}7.439 & 0.037\end{array}$ & 14464 & $\mathrm{GH}$ & 7.332 & 0.035 \\
\hline 14966 & $\mathrm{GH}$ & 7.1150 .033 & 14465 & $\mathrm{GH}$ & 7.068 & 0.033 \\
\hline 14967 & $\mathrm{GH}$ & $\begin{array}{lll}7.051 & 0.035\end{array}$ & 14466 & $\mathrm{GH}$ & 7.047 & 0.036 \\
\hline 14968 & $\mathrm{GH}$ & $6.744 \quad 0.036$ & 14367 & $\mathrm{GH}$ & 6.832 & 0.039 \\
\hline 14969 & $\mathrm{GH}$ & $6.580 \quad 0.033$ & 14368 & $\mathrm{GH}$ & 6.626 & 0.037 \\
\hline 14970 & $\mathrm{GH}$ & $\begin{array}{lll}6.273 & 0.032\end{array}$ & 14369 & $\mathrm{GH}$ & 311 & 0.034 \\
\hline 14971 & $\mathrm{GH}$ & 6.0920 .030 & 14370 & $\mathrm{GH}$ & 34 & 0.032 \\
\hline 14972 & $\mathrm{GH}$ & $\begin{array}{lll}5.757 & 0.031\end{array}$ & 14270 & $\mathrm{GH}$ & 6.064 & 0.032 \\
\hline 14973 & $\mathrm{GH}$ & $5.574 \quad 0.030$ & 14273 & $\mathrm{GH}$ & 5.516 & 0.028 \\
\hline 14974 & $\mathrm{GH}$ & 5.2240 .030 & 14173 & $\mathrm{GH}$ & 5.433 & 0.030 \\
\hline 14975 & $F G^{*}$ & $4.703 \quad 0.026$ & 14174 & $\mathrm{GH}$ & 4.899 & 0.026 \\
\hline 14976 & $\mathrm{GH}$ & $\begin{array}{lll}4.128 & 0.024\end{array}$ & 14175 & $\mathrm{GH}$ & 4.365 & 0.024 \\
\hline 14977 & $\mathrm{GH}$ & 3.5190 .022 & 14176 & $\mathrm{GH}$ & 3.773 & 0.026 \\
\hline & & & 14077 & GH & 3.075 & 0.021 \\
\hline
\end{tabular}

an-drawer column which designates the foil location in the drawer. The ${ }^{239} \mathrm{Pu}$ foils were centered $13.8 \mathrm{~mm}$ below the mid-height of the drawer. All foils were centered $51.6 \mathrm{~mm}$ from the reactor midplane except "*" which were at $50.3 \mathrm{~mm}$.

$b_{\text {Experimental results in units of } 10^{-1}}$ fissions per atom per second at a reactor power of approximately one watt. The second number is one standard deviation uncertainty. See text for details. 
TABLE 5.2 Basic Data for Radial Distributions of ${ }^{235} \mathrm{U}$ and ${ }^{238} \mathrm{U}$ Reaction Rates in ZPPR-18A

\begin{tabular}{|c|c|c|c|c|}
\hline Matrix & Loc & $235 U(n, f)^{b}$ & ${ }^{238} U(n, f)^{b}$ & $2^{38} \mathrm{U}(n, \gamma)^{\mathrm{b}}$ \\
\hline$x$-axis & lata at & $z=51.6 \mathrm{~mm}$ & & \\
\hline 14950 & GH & $9.266 \quad 0.037$ & $0.2253 \quad 0.0029$ & 1.31950 .0077 \\
\hline 14951 & GH & 9.0260 .038 & 0.21830 .0025 & $1.1308 \quad 0.0061$ \\
\hline 14952 & $\mathrm{GH}$ & 9.1750 .038 & 0.22030 .0026 & $1.3016 \quad 0.0070$ \\
\hline 14953 & GH & 8.9100 .038 & $0.2144 \quad 0.0026$ & 1.10490 .0066 \\
\hline 14954 & GH & 9.2190 .050 & 0.21530 .0023 & 1.26920 .0073 \\
\hline 14955 & $\mathrm{GH}$ & $\begin{array}{lll}8.771 & 0.040\end{array}$ & 0.20920 .0026 & 1.08520 .0060 \\
\hline 14956 & $\mathrm{GH}$ & $8.922 \quad 0.042$ & $0.2145 \quad 0.0027$ & 1.26370 .0067 \\
\hline 14957 & GH & $8.674 \quad 0.040$ & $0.2099 \quad 0.0025$ & $1.0716 \quad 0.0064$ \\
\hline 14958 & $\mathrm{GH}$ & $\begin{array}{lll}8.833 & 0.039\end{array}$ & 0.20810 .0028 & $1.2588 \quad 0.0068$ \\
\hline 14959 & GH & 8.6640 .041 & $0.2025 \quad 0.0027$ & 1.24040 .0071 \\
\hline 14960 & GH & 8.2740 .035 & 0.19790 .0026 & $1.0373 \quad 0.0058$ \\
\hline 14961 & GH & $\begin{array}{lll}8.417 & 0.037\end{array}$ & 0.19160 .0027 & 1.20370 .0063 \\
\hline $149 \quad 62$ & $\mathrm{GH}$ & $8.354 \quad 0.036$ & 0.19540 .0029 & $1.1954 \quad 0.0067$ \\
\hline 14963 & $\mathrm{GH}$ & 8.1120 .037 & 0.19110 .0033 & 1.01630 .0057 \\
\hline 14964 & GH & 8.0690 .035 & 0.19120 .0028 & 1.15780 .0068 \\
\hline 14965 & GH & 7.9070 .036 & 0.18190 .0032 & 1.14240 .0068 \\
\hline 14966 & $\mathrm{GH}$ & $\begin{array}{lll}7.497 & 0.032\end{array}$ & 0.18020 .0023 & 0.93490 .0054 \\
\hline 14967 & $\mathrm{GH}$ & $7.444 \quad 0.032$ & $0.1826 \quad 0.0024$ & 1.05630 .0059 \\
\hline 14968 & GH & $\begin{array}{lll}7.088 & 0.034\end{array}$ & 0.16410 .0025 & 0.88410 .0048 \\
\hline 14969 & $\mathrm{GH}$ & 6.9870 .031 & 0.16660 .0025 & $0.9900 \quad 0.0057$ \\
\hline 14970 & GH & $6.599 \quad 0.032$ & & \\
\hline 14971 & $\mathrm{GH}$ & 6.4720 .029 & $\begin{array}{lll}0.1585 & 0.0023\end{array}$ & $0.9060 \quad 0.0058$ \\
\hline 14972 & GH & $\begin{array}{lll}6.066 & 0.028\end{array}$ & $0.1504 \quad 0.0024$ & $0.7519 \quad 0.0044$ \\
\hline 14973 & $\mathrm{GH}$ & $\begin{array}{lll}5.865 & 0.028\end{array}$ & $\begin{array}{lll}0.1487 & 0.0023\end{array}$ & $\begin{array}{llll}0.8260 & 0.0051\end{array}$ \\
\hline 14974 & GH & $5.470 \quad 0.026$ & $0.1493 \quad 0.0022$ & $0.7701 \quad 0.0044$ \\
\hline 14976 & GH & $4.404 \quad 0.024$ & 0.11220 .0021 & $0.6156 \quad 0.0040$ \\
\hline 14977 & GH & $3.797 \quad 0.019$ & $0.0901 \quad 0.0023$ & 0.54130 .0046 \\
\hline 14978 & GH & $3.227 \quad 0.017$ & 0.04010 .0013 & 0.39390 .0033 \\
\hline 14979 & $\mathrm{GH}$ & 2.6550 .015 & 0.02050 .0012 & $0.3138 \quad 0.0024$ \\
\hline $149: 80$ & $\mathrm{GH}$ & 2.1320 .013 & 0.01210 .0009 & 0.24920 .0021 \\
\hline 14981 & $\mathrm{GH}$ & $1.738 \quad 0.011$ & $\begin{array}{lll}0.0060 & 0.0008\end{array}$ & 0.19350 .0018 \\
\hline 14982 & $\mathrm{GH}$ & 1.4860 .010 & 0.00490 .0009 & 0.15300 .0016 \\
\hline
\end{tabular}

$x$-axis data at $z=280.2 \mathrm{~mm}$

$\begin{array}{lllllllll}149 & 50 & \mathrm{GH} & 7.778 & 0.037 & 0.1739 & 0.0027 & 1.1210 & 0.0061 \\ 149 & 51 & \mathrm{GH} & 7.488 & 0.035 & 0.1723 & 0.0027 & 0.9392 & 0.0053 \\ 149 & 52 & \mathrm{GH} & 7.622 & 0.037 & 0.1769 & 0.0027 & 1.0880 & 0.0059 \\ 149 & 53 & \mathrm{GH} & 7.359 & 0.034 & 0.1704 & 0.0031 & 0.9228 & 0.0052 \\ 149 & 54 & \mathrm{GH} & 7.485 & 0.038 & 0.1688 & 0.0027 & 1.0659 & 0.0061 \\ 149 & 55 & \mathrm{GH} & 7.356 & 0.036 & 0.1617 & 0.0025 & 0.9049 & 0.0051 \\ 149 & 56 & \mathrm{GH} & 7.338 & 0.034 & 0.1695 & 0.0028 & 1.0648 & 0.0057 \\ 149 & 57 & \mathrm{GH} & 7.226 & 0.034 & 0.1666 & 0.0025 & 0.9005 & 0.0051 \\ 149 & 58 & \mathrm{GH} & 7.374 & 0.036 & 0.1633 & 0.0024 & 1.0496 & 0.0056\end{array}$


TABLE 5.2 (contd)

\begin{tabular}{|c|c|c|c|c|c|}
\hline Matrix & $\operatorname{Loc}^{2}$ & $235 \mathrm{U}(\mathrm{n}, \mathrm{f})^{\mathrm{b}}$ & ${ }^{238} U(n, f)^{b}$ & $238 \mathrm{U}($ & $(1, \gamma)^{b}$ \\
\hline 14959 & $\mathrm{GH}$ & $\begin{array}{lll}7.157 & 0.037\end{array}$ & $\begin{array}{lll}0.1650 & 0.0029\end{array}$ & 1.0268 & 0.0061 \\
\hline 14960 & $\mathrm{GH}$ & 6.9010 .033 & 0.15520 .0031 & 0.8681 & 0.0050 \\
\hline 14961 & GH & 6.9530 .034 & 0.16350 .0022 & 0.9828 & 0.0055 \\
\hline 14962 & $\mathrm{GH}$ & $6.953 \quad 0.033$ & 0.15830 .0030 & 0.9909 & 0.0054 \\
\hline 14963 & $\mathrm{GH}$ & $6.694 \quad 0.032$ & 0.15390 .0023 & 0.8320 & 0.0047 \\
\hline 149.64 & GH & $\begin{array}{lll}6.687 & 0.031\end{array}$ & 0.16260 .0025 & 0.9461 & 0.0054 \\
\hline 14965 & $\mathrm{GH}$ & $\begin{array}{ll}6.653 & 0.031\end{array}$ & $0.1500 \quad 0.0026$ & 0.9495 & 0.0053 \\
\hline 14966 & $\mathrm{GH}$ & $6.272 \quad 0.031$ & 0.14320 .0020 & 0.7817 & 0.0049 \\
\hline 14967 & $\mathrm{GH}$ & $6.190 \quad 0.031$ & $0.1464 \quad 0.0022$ & 0.8788 & 0.0052 \\
\hline 14968 & $\mathrm{GH}$ & $5.858 \quad 0.030$ & $\begin{array}{lll}0.1378 & 0.0021\end{array}$ & 0.7230 & 0.0044 \\
\hline 14969 & $\mathrm{GH}$ & $5.745 \quad 0.030$ & 0.14290 .0022 & 0.8122 & 0.0048 \\
\hline 14970 & GH & $5.421 \quad 0.027$ & $0.1270 \quad 0.0024$ & 0.6767 & 0.0040 \\
\hline 14971 & GH & 5.2950 .026 & 0.12410 .0022 & 0.7594 & 0.0045 \\
\hline 14972 & $\mathrm{GH}$ & 4.9410 .027 & 0.12050 .0024 & 0.6195 & 0.0038 \\
\hline 149.73 & $\mathrm{GH}$ & 4.8390 .027 & 0.12370 .0026 & 0.6810 & 0.0041 \\
\hline 14974 & $\mathrm{GH}$ & 4.4810 .024 & & & \\
\hline 14976 & $\mathrm{GH}$ & 3.5780 .020 & & & \\
\hline 14977 & $\mathrm{GH}$ & $3.096 \quad 0.017$ & $0.0720 \quad 0.0020$ & 0.4468 & 0.0031 \\
\hline 14978 & $\mathrm{GH}$ & $2.652 \quad 0.018$ & & & \\
\hline 14979 & $\mathrm{GH}$ & $2.190 \quad 0.013$ & & & \\
\hline 14980 & GH & $1.744 \quad 0.012$ & & & \\
\hline 14981 & $\mathrm{GH}$ & $1.418 \quad 0.011$ & & & \\
\hline 14982 & $\mathrm{GH}$ & 1.2190 .009 & & & \\
\hline $15^{\circ} \mathrm{d}$ & & $51.6 \mathrm{~mm}$ & & & \\
\hline 14850 & GH & 9.2110 .040 & 0.22350 .0024 & & 0.0069 \\
\hline 14852 & $\mathrm{GH}$ & 8.9590 .038 & $0.2126 \quad 0.0026$ & 1.1106 & 0.0061 \\
\hline 14853 & $\mathrm{GH}$ & 9.1090 .038 & 0.21650 .0026 & 1.2826 & 0.0075 \\
\hline 14754 & $\mathrm{GH}$ & 9.1830 .043 & $0.2107 \quad 0.0026$ & 1.2673 & 0.0065 \\
\hline 14755 & $\mathrm{GH}$ & $8.807 \quad 0.040$ & 0.20620 .0026 & 1.0976 & 0.0065 \\
\hline 14756 & $\mathrm{GH}$ & 9.0240 .042 & $0.2115 \quad 0.0026$ & 1.2906 & 0.0069 \\
\hline 14757 & $\mathrm{GH}$ & 9.0190 .041 & 0.20620 .0025 & 1.2749 & 0.0068 \\
\hline 14657 & $\mathrm{GH}^{*}$ & & $0.1875 \quad 0.0026$ & 1.1027 & 0.0058 \\
\hline 14658 & $\mathrm{GH}$ & 9.0010 .043 & 0.19820 .0026 & 1.2704 & 0.0068 \\
\hline 14659 & $\mathrm{GH}$ & $8.447 \quad 0.038$ & $0.1873 \quad 0.0027$ & 1.0590 & 0.0058 \\
\hline 146.60 & $\mathrm{GH}$ & 8.4430 .035 & 0.19290 .0024 & 1.1985 & 0.0071 \\
\hline 14560 & $\mathrm{GH}$ & 8.2270 .036 & $0.1851 \quad 0.0027$ & 1.0194 & 0.0060 \\
\hline 14561 & $\mathrm{GH}$ & $8.221 \quad 0.034$ & $0.1930 \quad 0.0029$ & 1.1830 & 0.0066 \\
\hline 145.62 & $\mathrm{GH}$ & $7.947 \quad 0.035$ & 0.18330 .0029 & 0.9968 & 0.0056 \\
\hline 14463 & $\mathrm{GH}$ & 7.7130 .033 & $0.1826 \quad 0.0030$ & 0.9708 & 0.0061 \\
\hline 14464 & $\mathrm{GH}$ & $\begin{array}{lll}7.781 & 0.037\end{array}$ & 0.18240 .0026 & 1.0963 & 0.0060 \\
\hline 14465 & $\mathrm{GH}$ & $\begin{array}{lll}7.417 & 0.034\end{array}$ & $0.1801 \quad 0.0030$ & 0.9201 & 0.0059 \\
\hline 14466 & $\mathrm{GH}$ & $\begin{array}{lll}7.461 & 0.033\end{array}$ & 0.17860 .0025 & 1.0522 & 0.0059 \\
\hline 14367 & $\mathrm{GH}$ & 7.1910 .034 & $0.1650 \quad 0.0023$ & 1.0302 & 0.0055 \\
\hline 14368 & $\mathrm{GH}$ & $\begin{array}{lll}7.038 & 0.032\end{array}$ & $0.1630 \quad 0.0023$ & 1.0004 & 0.0057 \\
\hline 14369 & GH & 6.6680 .030 & $\begin{array}{lll}0.1552 & 0.0031\end{array}$ & 0.8322 & 0.0052 \\
\hline 14370 & $\mathrm{GH}$ & $\begin{array}{lll}6.688 & 0.030\end{array}$ & 0.14420 .0026 & 0.9598 & 0.0055 \\
\hline
\end{tabular}


TABLE 5.2 (contd)

\begin{tabular}{|c|c|c|c|c|}
\hline Matrix & $\operatorname{Loc}^{a}$ & $235 U(n, f)^{b}$ & ${ }^{238} U(n, f)^{b}$ & $23 a U(n, \gamma)^{b}$ \\
\hline 14270 & $\mathrm{GH}$ & 6.4420 .028 & 0.13790 .0022 & 0.79320 .0045 \\
\hline 14273 & $\mathrm{GH}$ & 5.9350 .031 & $\begin{array}{lll}0.1342 & 0.0021\end{array}$ & 0.85330 .0047 \\
\hline 14173 & $\mathrm{GH}$ & $5.703 \quad 0.025$ & 0.13240 .0024 & 0.83360 .0048 \\
\hline 14174 & $\mathrm{GH}$ & 5.1230 .024 & $\begin{array}{lll}0.1346 & 0.0021\end{array}$ & 0.73430 .0043 \\
\hline 14175 & $\mathrm{GH}$ & $\begin{array}{lll}4.525 & 0.022\end{array}$ & $0.1280 \quad 0.0022$ & 0.64030 .0044 \\
\hline 14176 & $\mathrm{GH}$ & 3.9190 .021 & 0.10530 .0019 & 0.55300 .0036 \\
\hline 14077 & $\mathrm{GH}$ & $3.215 \quad 0.017$ & 0.08290 .0017 & 0.46720 .0033 \\
\hline 14078 & $\mathrm{GH}$ & 2.7470 .015 & 0.03670 .0012 & $0.3340 \quad 0.0027$ \\
\hline 14079 & $\mathrm{GH}$ & 2.2250 .013 & 0.01810 .0010 & 0.26890 .0022 \\
\hline 13980 & $\mathrm{GH}$ & $\begin{array}{lll}1.682 & 0.011\end{array}$ & 0.01140 .0010 & $0.1988 \quad 0.0021$ \\
\hline 13981 & $\mathrm{GH}$ & 1.3490 .009 & 0.00690 .0008 & 0.15320 .0016 \\
\hline 13982 & $\mathrm{GH}$ & 1.1400 .008 & $0.0050 \quad 0.0008$ & 0.11720 .0014 \\
\hline \\
\hline 14850 & GH & $7.709 \quad 0.035$ & 0.17600 .0027 & 1.10150 .0060 \\
\hline 14852 & GH & 7.4340 .034 & 0.16990 .0028 & $0.9259 \quad 0.0057$ \\
\hline 14853 & $\mathrm{GH}$ & 7.5630 .035 & $\begin{array}{llll}0.1738 & 0.0026\end{array}$ & $1.0788 \quad 0.0064$ \\
\hline 14754 & $\mathrm{GH}$ & $7.478 \quad 0.035$ & 0.17150 .0025 & 1.07550 .0058 \\
\hline 14755 & $\mathrm{GH}$ & $\begin{array}{lll}7.335 & 0.037\end{array}$ & & \\
\hline 14756 & $\mathrm{GH}$ & $7.488 \quad 0.037$ & 0.16610 .0026 & $1.0744 \quad 0.0058$ \\
\hline 14757 & $\mathrm{GH}$ & $7.450 \quad 0.035$ & 0.16450 .0026 & $1.0794 \quad 0.0058$ \\
\hline $146 \quad 57$ & $\mathrm{GH}$ & & 0.15120 .0026 & 0.91470 .0051 \\
\hline 14658 & $\mathrm{GH}$ & $\begin{array}{lll}7.428 & 0.033\end{array}$ & 0.16140 .0023 & 1.06000 .0059 \\
\hline 14659 & $\mathrm{GH}$ & $7.033 \quad 0.034$ & $\begin{array}{llll}0.1514 & 0.0028\end{array}$ & 0.87890 .0053 \\
\hline 14660 & $\mathrm{GH}$ & 7.0190 .033 & & \\
\hline 14560 & $\mathrm{GH}$ & 6.8720 .033 & 0.15220 .0028 & 0.85410 .0051 \\
\hline 14561 & $\mathrm{GH}$ & $\begin{array}{lll}6.772 & 0.029\end{array}$ & & \\
\hline 14562 & $\mathrm{GH}$ & $\begin{array}{lll}6.528 & 0.032\end{array}$ & 0.15210 .0025 & 0.82040 .0047 \\
\hline 14463 & $\mathrm{GH}$ & $\begin{array}{lll}6.402 & 0.031\end{array}$ & $\begin{array}{llll}0.1424 & 0.0024\end{array}$ & $0.7990 \quad 0.0046$ \\
\hline 14464 & $\mathrm{GH}$ & 6.4260 .031 & & \\
\hline 14465 & $\mathrm{GH}$ & 6.1690 .029 & 0.14170 .0023 & 0.76330 .0048 \\
\hline 14466 & $\mathrm{GH}$ & $\begin{array}{lll}6.141 & 0.028\end{array}$ & & \\
\hline 14367 & $\mathrm{GH}$ & $\begin{array}{lll}5.935 & 0.030\end{array}$ & $\begin{array}{lll}0.1385 & 0.0021\end{array}$ & $0.8470 \quad 0.0049$ \\
\hline 14368 & GH & $5.794 \quad 0.029$ & & \\
\hline 14369 & $\mathrm{GH}$ & $5.524 \quad 0.027$ & $0.1248 \quad 0.0024$ & $0.6826 \quad 0.0041$ \\
\hline 14370 & $\mathrm{GH}$ & $\begin{array}{lll}5.517 & 0.028\end{array}$ & $\begin{array}{lll}0.1171 & 0.0021\end{array}$ & 0.79230 .0045 \\
\hline 14270 & $\mathrm{GH}$ & $5.353 \quad 0.027$ & 0.11380 .0022 & 0.66140 .0039 \\
\hline 14273 & $\mathrm{GH}$ & $\begin{array}{lll}4.915 & 0.024\end{array}$ & $0.1096 \quad 0.0022$ & $0.7077 \quad 0.0047$ \\
\hline 14173 & $\mathrm{GH}$ & $4.786 \quad 0.023$ & 0.10780 .0023 & $0.6957 \quad 0.0042$ \\
\hline 14174 & $\mathrm{GH}$ & $4.220 \quad 0.021$ & 0.10710 .0022 & $0.6020 \quad 0.0038$ \\
\hline 14175 & $\mathrm{GH}$ & $\begin{array}{lll}3.727 & 0.023\end{array}$ & & \\
\hline 14176 & $\mathrm{GH}$ & 3.2350 .019 & $\begin{array}{lll}0.0861 & 0.0017\end{array}$ & $0.4552 \quad 0.0032$ \\
\hline 14077 & $\mathrm{GH}$ & 2.6820 .018 & $\begin{array}{lll}0.0647 & 0.0017\end{array}$ & 0.38140 .0028 \\
\hline 14078 & $\mathrm{GH}$ & 2.2260 .016 & & \\
\hline 14079 & $\therefore \mathrm{GH}$ & $\begin{array}{lll}1.811 & 0.012\end{array}$ & & \\
\hline 13980 & $\therefore \quad \mathrm{GH}$ & $1.370 \quad 0.009$ & & \\
\hline 13981 & $\mathrm{GH}$ & 1.0940 .008 & & \\
\hline 13982 & $\mathrm{GH}$ & 0.9310 .008 & & \\
\hline
\end{tabular}


TABLE 5.2 (contd)

\begin{tabular}{|c|c|c|c|c|}
\hline Matrix & Loc ${ }^{a}$ & $235 U(n, f)^{b}$ & $238 \mathrm{U}(n, f)^{b}$ & $238 \mathrm{U}(n, \gamma)^{b}$ \\
\hline \multicolumn{5}{|c|}{$30^{\circ}$ data at $\mathrm{z}=51.6 \mathrm{~mm}$} \\
\hline 14654 & $\mathrm{GH}$ & $\begin{array}{lll}8.870 & 0.040\end{array}$ & $0.1996 \quad 0.0031$ & 1.10260 .0062 \\
\hline 14555 & $\mathrm{GH}$ & $8.940 \quad 0.041$ & $0.1890 \quad 0.0026$ & 1.11280 .0063 \\
\hline 14458 & $\mathrm{GH}$ & 8.8120 .038 & $0.1782 \quad 0.0029$ & 1.09480 .0060 \\
\hline 14358 & $\mathrm{GH}$ & 8.8310 .042 & 0.18250 .0025 & $1.2956 \quad 0.0066$ \\
\hline 14359 & $\mathrm{GH}$ & $8.273 \quad 0.037$ & 0.18130 .0029 & 1.03470 .0056 \\
\hline 14260 & GH & $7.925 \quad 0.035$ & $0.1843 \cdot 0.0026$ & 0.99390 .0057 \\
\hline 14261 & $\mathrm{GH}$ & $7.996 \quad 0.038$ & $0.1807 \quad 0.0028$ & $1.1524 \quad 0.0061$ \\
\hline 14262 & $\mathrm{GH}$ & 8.0210 .036 & $0.1755 \quad 0.0024$ & 1.15260 .0064 \\
\hline 14162 & $\mathrm{GH}$ & 7.9570 .036 & $0.1716 \quad 0.0025$ & $1.1479 \quad 0.0061$ \\
\hline 14065 & $\mathrm{GH}$ & $7.375 \quad 0.034$ & $0.1506 \quad 0.0023$ & 0.91960 .0051 \\
\hline 13965 & $\mathrm{GH}$ & $7.381 \quad 0.035$ & 0.15120 .0025 & 1.09190 .0061 \\
\hline 13966 & $\mathrm{GH}$ & $6.806 \quad 0.035$ & $0.1562 \quad 0.0024$ & $0.8423 \quad 0.0054$ \\
\hline 13867 & GH & 6.4630 .035 & 0.15510 .0022 & 0.79910 .0051 \\
\hline 13868 & $\mathrm{GH}$ & $6.386 \quad 0.031$ & 0.16140 .0023 & $0.9021 \cdot 0.0052$ \\
\hline 13768 & $\mathrm{GH}$ & $6.126 \quad 0.031$ & 0.15670 .0023 & 0.76370 .0047 \\
\hline \multicolumn{5}{|c|}{$30^{\circ}$ data at $\mathrm{z}=280.2 \mathrm{~mm}$} \\
\hline 14654 & $\mathrm{GH}$ & 7.3470 .035 & & \\
\hline 14555 & $\mathrm{GH}$ & $7.520 \quad 0.035$ & & \\
\hline 14458 & $\mathrm{GH}$ & $7.298 \quad 0.037$ & & \\
\hline 14358 & GH & $7.398 \quad 0.038$ & & \\
\hline 14359 & $\mathrm{GH}$ & 6.8810 .032 & & \\
\hline 14260 & $\mathrm{GH}$ & 6.6140 .030 & & \\
\hline 14261 & $\mathrm{GH}$ & 6.7020 .031 & & \\
\hline 14262 & GH & $6.646 \quad 0.034$ & & \\
\hline 14162 & $\mathrm{GH}$ & 6.5960 .029 & & \\
\hline 14065 & $\mathrm{GH}$ & $6.148 \quad 0.028$ & & \\
\hline 13965 & GH & 6.1920 .031 & & \\
\hline 13966 & $\mathrm{GH}$ & 5.7190 .032 & & \\
\hline 13867 & GH & 5.2620 .028 & & \\
\hline 13868 & $\mathrm{GH}$ & 5.2490 .029 & & \\
\hline 13768 & $\mathrm{GH}$ & 5.0060 .026 & & \\
\hline \multicolumn{5}{|c|}{$y$-axis data at $z=51.6 \mathrm{~mm}$} \\
\hline 14849 & $\mathrm{GH}$ & 9.1270 .041 & & \\
\hline 14749 & GH & 9.1670 .039 & & \\
\hline $146 \quad 49$ & GH & 8.9190 .037 & & \\
\hline $145 \quad 49$ & GH & 9.1830 .042 & & \\
\hline $144 \quad 49$ & GH & $9.001 \quad 0.038$ & & \\
\hline 14349 & GH & $8.764 \quad 0.039$ & & \\
\hline 14249 & GH & 8.8810 .038 & & \\
\hline 14149 & GH & $8.795 \quad 0.041$ & & \\
\hline $138 \cdot 49$ & $\mathrm{GH}$ & $8.338 \quad 0.038$ & & \\
\hline 13749 & $\mathrm{GH}$ & $7.844 \quad 0.036$ & & \\
\hline 13649 & GH & $7.810 \quad 0.035$ & & \\
\hline
\end{tabular}


TABLE 5.2 (contd)

\begin{tabular}{|c|c|c|c|c|}
\hline Matr1x & $\operatorname{Loc}^{a}$ & $2{ }^{5} U(n, f)^{b}$ & ${ }^{23} \mathrm{U}(n, f)^{b}$ & ${ }^{238} U(n, \gamma)^{b}$ \\
\hline 13549 & GH & 7.6270 .036 & & \\
\hline 13449 & $\mathrm{GH}$ & $7.363 \quad 0.034$ & & \\
\hline 13349 & $\mathrm{GH}$ & $7.460 \quad 0.033$ & & \\
\hline 13049 & $\mathrm{GH}$ & $\begin{array}{lll}6.578 & 0.032\end{array}$ & & \\
\hline $129 \quad 49$ & GH & $\begin{array}{lll}6.327 & 0.029\end{array}$ & & \\
\hline $128 \quad 49$ & $\mathrm{GH}$ & $\begin{array}{lll}5.868 & 0.029\end{array}$ & & \\
\hline $127 \quad 49$ & $\mathrm{GH}$ & $5.706 \quad 0.026$ & & \\
\hline 12649 & $\mathrm{GH}$ & $\begin{array}{llll}5.410 & 0.025\end{array}$ & & \\
\hline 12549 & $C D$ & $4.976 \quad 0.029$ & 0.14760 .0023 & 0.67300 .0040 \\
\hline $124 \quad 49$ & HI & $4.548 \quad 0.026$ & $0.1394 \quad 0.0021$ & 0.61330 .0040 \\
\hline 12349 & $C D$ & $\begin{array}{lll}4.173 & 0.023\end{array}$ & 0.12870 .0022 & $0.5670 \quad 0.0041$ \\
\hline 12249 & $\mathrm{HI}$ & $3.707 \quad 0.023$ & 0.11310 .0019 & $0.5045 \quad 0.0035$ \\
\hline 12149 & HI & $3.178 \quad 0.020$ & 0.09890 .0018 & 0.43540 .0032 \\
\hline $120 \quad 49$ & $C D$ & 2.6680 .015 & $0.0784 \quad 0.0018$ & $0.3758 \quad 0.0029$ \\
\hline 11949 & $\mathrm{HI}$ & $2.211 \quad 0.015$ & $0.0580 \quad 0.0016$ & $0.3067 \quad 0.0027$ \\
\hline
\end{tabular}

an-drawer column which designates the foll location in the drawer. The ${ }^{235} \mathrm{U}$ folls were centered $13.8 \mathrm{~mm}$ above and the ${ }^{23} \mathrm{U}$ foils were centered on the mid-height of the drawer. All foils were centered $51.6 \mathrm{~mm}$ from the reactor midplane except "*" which were at 50.3.

Experimental results in units of $10^{-18}$ fissions or captures per atom per second at a reactor power of approximately one watt. The second number is one standard deviation of uncertainty. See text for details. 
TABLE 5.3 Basic Reaction Rate Data for Axial Distributions in ZPPR-18A

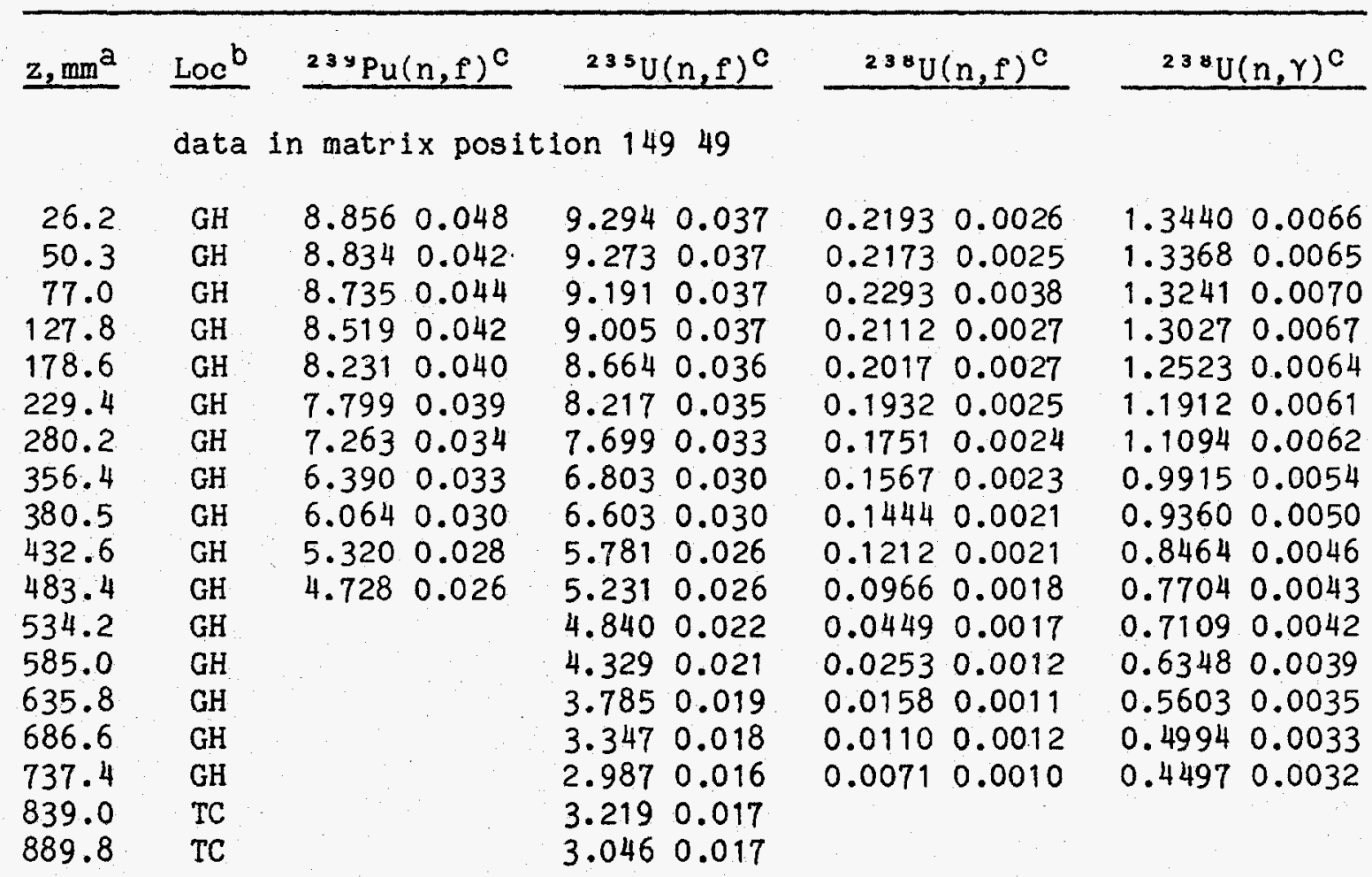

data in matrix position 14975

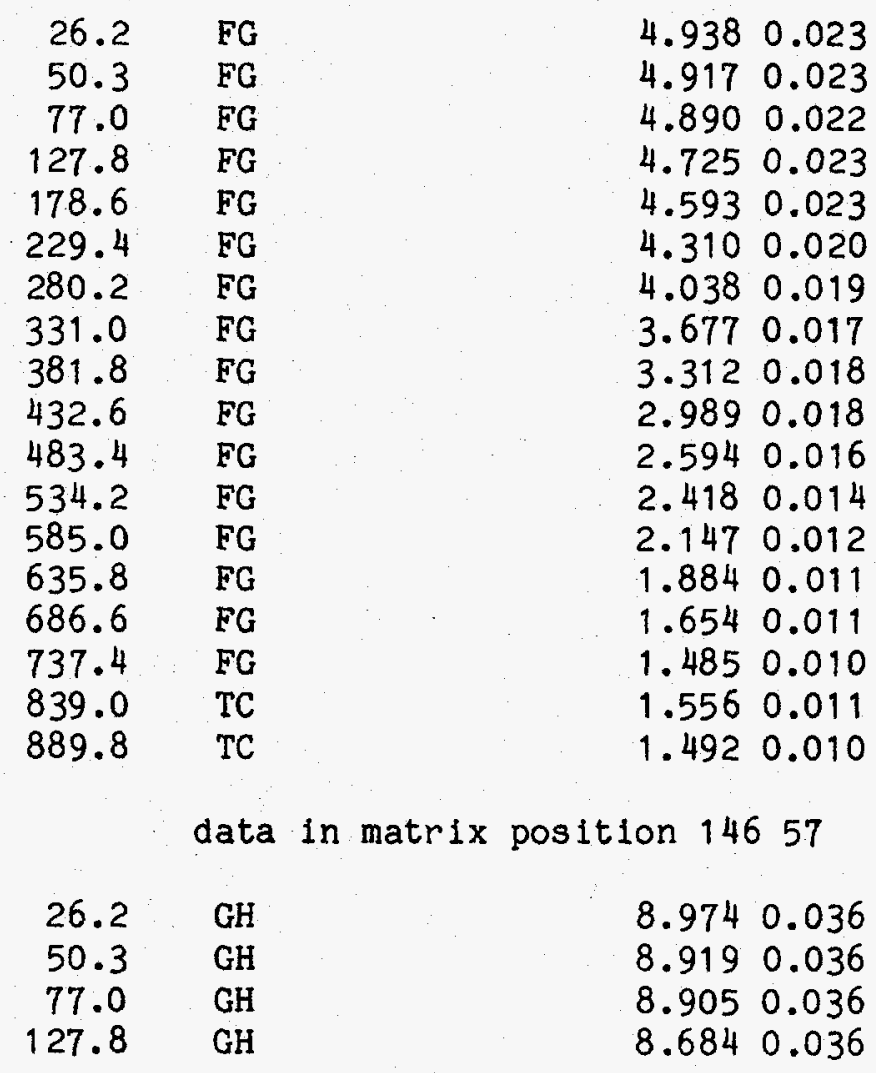

$\begin{array}{llllll}0.1455 & 0.0021 & 0.7037 & 0.0040\end{array}$

$\begin{array}{llllll}0.1438 & 0.0023 & 0.6969 & 0.0040\end{array}$

$\begin{array}{lllll}0.1450 & 0.0028 & 0.6921 & 0.0046\end{array}$

$\begin{array}{llllll}0.1377 & 0.0028 & 0.6745 & 0.0041\end{array}$

$\begin{array}{lllll}0.1316 & 0.0023 & 0.6465 & 0.0040\end{array}$

$\begin{array}{lllll}0.1248 & 0.0023 & 0.6141 & 0.0038\end{array}$

$\begin{array}{lllll}0.1163 & 0.0024 & 0.5694 & 0.0038\end{array}$

$\begin{array}{lllll}0.1040 & 0.0025 & 0.5233 & 0.0038\end{array}$

$\begin{array}{lllll}0.0904 & 0.0022 & 0.4769 & 0.0032\end{array}$

$\begin{array}{llll}0.0790 & 0.0019 & 0.4283 & 0.0030\end{array}$

$\begin{array}{lllll}0.0610 & 0.0017 & 0.3838 & 0.0029\end{array}$

$\begin{array}{lllll}0.0284 & 0.0014 & 0.3309 & 0.0025\end{array}$

$\begin{array}{llllll}0.0149 & 0.0011 & 0.2937 & 0.0027\end{array}$

$0.0100 \quad 0.0011 \quad 0.25460 .0022$

$\begin{array}{llll}0.0070 & 0.0009 & 0.2210 & 0.0020\end{array}$ 
TABLE 5.3 (contd)

\begin{tabular}{|c|c|c|c|c|c|}
\hline$\underline{\mathrm{z}}, \mathrm{mm}^{\mathrm{a}}$ & Loc ${ }^{b}$ & ${ }^{2{ }^{3 y} \mathrm{Pu}(\mathrm{n}, \mathrm{f})^{\mathrm{C}}}$ & ${ }^{235} \mathrm{U}(n, f)^{\mathrm{c}}$ & $2^{3 y} U(n, f)^{\mathrm{C}}$ & ${ }^{2{ }^{3} \mathrm{U}} \mathrm{U}(n, \gamma)^{\mathrm{C}}$ \\
\hline 178.6 & $\mathrm{GH}$ & & $8.290 \quad 0.036$ & & \\
\hline 229.4 & $\mathrm{GH}$ & & $7.916 \quad 0.034$ & & \\
\hline 280.2 & GH & & 7.4360 .031 & & \\
\hline 331.0 & $\mathrm{GH}$ & & $\begin{array}{lll}6.848 & 0.031\end{array}$ & & \\
\hline 381.8 & $\mathrm{GH}$ & & $6.310 \quad 0.028$ & & \\
\hline 432.6 & $\mathrm{GH}$ & & $5.690 \quad 0.027$ & & \\
\hline 483.4 & $\mathrm{GH}$ & & $\begin{array}{lll}5.183 & 0.024\end{array}$ & & \\
\hline 534.2 & $\mathrm{GH}$ & & 5.0260 .024 & & \\
\hline 585.0 & $\mathrm{GH}$ & & $4.580 \quad 0.021$ & & \\
\hline 635.8 & $\mathrm{GH}$ & & $\begin{array}{lll}4.088 & 0.021\end{array}$ & & \\
\hline 686.6 & $\mathrm{GH}$ & & $3.708 \quad 0.019$ & & \\
\hline 737.4 & $\mathrm{GH}$ & & $3.379 \quad 0.019$ & & \\
\hline
\end{tabular}

a Distance from the reactor interface to the center of the foll.

${ }^{b}$ In-drawer column which designates the foll location in the drawer. The ${ }^{235} \mathrm{U}$ foils were centered $13.8 \mathrm{~mm}$ above, the ${ }^{238} \mathrm{U}$ foils were centered on, and the ${ }^{23 y} \mathrm{Pu}$ foils were centered $13.8 \mathrm{~mm}$ below the mid-height of the drawer.

Experimental results in units of $10^{-1}$ \& fissions or captures per atom per second at a reactor power of approximately one watt. The second number is one standard deviation uncertainty. See text for details. 
TABLE 5.4 Basic Data for Radial Distributions of $2{ }^{3} \mathrm{Pu}(n, f)$ in $\mathrm{ZPPR}-18 \mathrm{~B}$

\begin{tabular}{|c|c|c|c|c|c|c|}
\hline Matrix & Loc & ${ }^{2{ }^{3} \mathrm{Pu}(n, f)^{\mathrm{b}}}$ & Matrix & Loc $^{b}$ & \multicolumn{2}{|c|}{$2{ }^{2{ }^{y}} \mathrm{Pu}(\mathrm{n}, \mathrm{f})$} \\
\hline$x^{+}$axis & data at & $z=51.6 \mathrm{~mm}^{\mathrm{c}}$ & $15^{\circ} \mathrm{d}$ & a at 2 & $=51.6$ & $m m^{c}$ \\
\hline 24950 & IJ & 8.3330 .040 & 24850 & $I J$ & 8.316 & 0.040 \\
\hline 24951 & I J & $8.075 \quad 0.040$ & 24852 & IJ & 7.973 & 0.038 \\
\hline 24952 & $I J$ & 8.1390 .038 & 24853 & IJ & 8.002 & 0.038 \\
\hline 24953 & $I J$ & 7.8550 .036 & 24754 & IJ & 7.688 & 0.037 \\
\hline 24954 & $I J$ & $7.864 \quad 0.040$ & 24756 & IJ & 7.350 & 0.034 \\
\hline 24955 & $I J$ & $\begin{array}{lll}7.579 & 0.038\end{array}$ & $247 \quad 57$ & IJ & 7.330 & 0.037 \\
\hline 24956 & IJ & $\begin{array}{lll}7.508 & 0.037\end{array}$ & 24657 & $I J^{*}$ & 6.771 & 0.034 \\
\hline 24957 & IJ & $\begin{array}{lll}7.450 & 0.037\end{array}$ & 24658 & IJ & 6.930 & 0.037 \\
\hline 24958 & $I J$ & $\begin{array}{lll}7.607 & 0.038\end{array}$ & 24659 & IJ & 7.082 & 0.037 \\
\hline 24959 & IJ & $7.616 \quad 0.038$ & 24660 & IJ & 7.417 & 0.038 \\
\hline 24960 & IJ & $7.485 \quad 0.038$ & 24560 & IJ & 7.146 & 0.037 \\
\hline 24961 & IJ & $\begin{array}{llll}7.680 & 0.040\end{array}$ & 24561 & IJ & 7.516 & 0.038 \\
\hline 24962 & IJ & $\begin{array}{lll}7.674 & 0.039\end{array}$ & 24562 & IJ & 7.300 & 0.037 \\
\hline 24963 & IJ & 7.5030 .042 & 24463 & $I J$ & 7.194 & 0.035 \\
\hline 24964 & $I J^{*}$ & 7.6430 .040 & 24464 & IJ & 7.260 & 0.037 \\
\hline 24965 & IJ & $7.468 \quad 0.038$ & $244 \quad 65$ & IJ & 7.032 & 0.035 \\
\hline 24966 & $\mathrm{IJ}$ & 7.1440 .036 & 24466 & $I J$ & 6.978 & 0.036 \\
\hline 24967 & IJ & 7.0490 .036 & 24367 & IJ & 6.565 & 0.034 \\
\hline 24968 & IJ & $\begin{array}{lll}6.651 & 0.035\end{array}$ & 24368 & IJ & 6.336 & 0.034 \\
\hline 24969 & IJ & $\begin{array}{llll}6.543 & 0.034\end{array}$ & 24369 & IJ & 5.757 & 0.032 \\
\hline 24970 & IJ & $\begin{array}{lll}6.115 & 0.033\end{array}$ & 24370 & IJ & 5.204 & 0.029 \\
\hline 24971 & $I J$ & $\begin{array}{lll}5.927 & 0.032\end{array}$ & 24270 & IJ & 4.899 & 0.028 \\
\hline 24972 & $I J$ & $\begin{array}{lll}5.529 & 0.031\end{array}$ & 24273 & IJ & 3.907 & 0.024 \\
\hline 24973 & IJ & $\begin{array}{lll}5.285 & 0.031\end{array}$ & 24173 & IJ & 3.898 & 0.024 \\
\hline 24974 & JK & $\begin{array}{lll}4.928 & 0.031\end{array}$ & 24174 & IJ & 3.913 & 0.024 \\
\hline 24975 & $\mathrm{JK}^{*}$ & 4.4430 .024 & 24175 & $\mathrm{JK}$ & 3.699 & 0.024 \\
\hline 24976 & $I J$ & 3.8250 .025 & 24176 & IJ & 3.298 & 0.021 \\
\hline 24977 & $I J$ & $3.290 \quad 0.021$ & 24077 & $I J$ & 2.715 & 0.019 \\
\hline
\end{tabular}

${ }^{a}$ In-drawer column which designates the foll location in the drawer. The ${ }^{23 y} \mathrm{Pu}$ folls were centered $13.8 \mathrm{~mm}$ below the mid-helght of the drawer. All foils were centered $51.6 \mathrm{~mm}$ from the reactor $\mathrm{midplane}$ except "*" which were at $50.3 \mathrm{~mm}$.

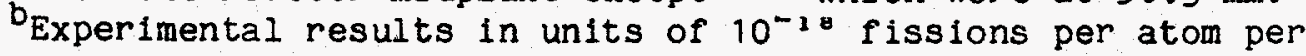
second at a reactor power of approximately one watt. The second number is one standard deviation uncertainty. See text for details. 
TABLE 5.5 Basic Data for Radial Distributions of ${ }^{235} \mathrm{U}(n, f)$ in Half 2 of $2 P P R-18 B$

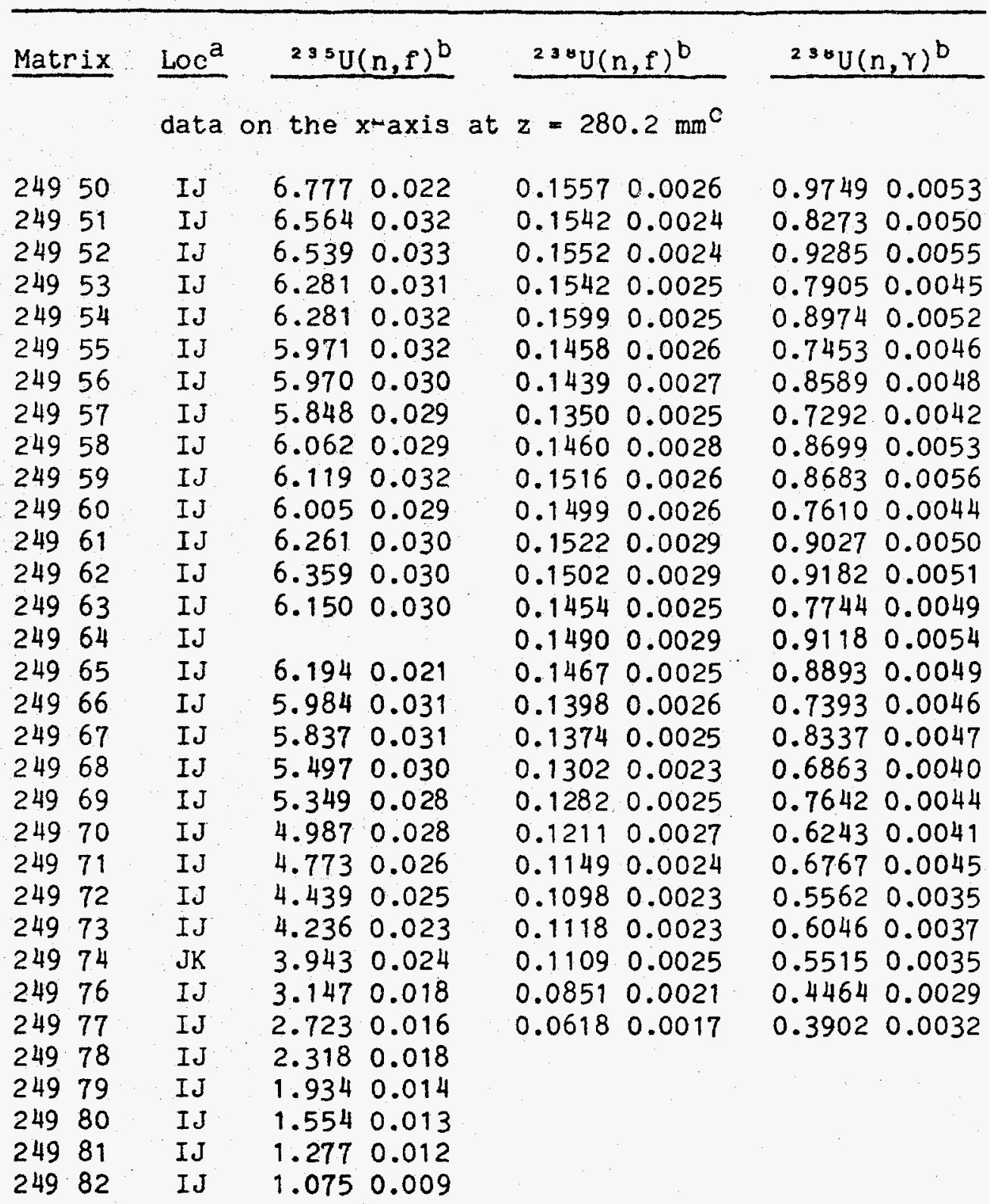

data on the $15^{\circ}$ radial at $z=280.2 \mathrm{~mm}^{\mathrm{C}}$

$\begin{array}{lllll}248 & 50 & \text { IJ } & 6.682 & 0.033 \\ 248 & 52 & \text { IJ } & 6.375 & 0.032 \\ 248 & 53 & \text { IJ } & 6.314 & 0.031 \\ 247 & 54 & \text { IJ } & 6.022 & 0.031 \\ 247 & 56 & \text { IJ } & 5.536 & 0.032 \\ 247 & 57 & \text { IJ } & 5.440 & 0.027 \\ 246 & 58 & \text { IJ } & 5.175 & 0.027 \\ 246 & 59 & \text { IJ } & 5.451 & 0.028 \\ 246 & 60 & \text { IJ } & 5.829 & 0.029 \\ 245 & 60 & \text { IJ } & 5.575 & 0.028 \\ 245 & 61 & \text { IJ } & 5.922 & 0.029\end{array}$

0.15940 .0025

0.15310 .0024

0.15310 .0023

0.14860 .0025

0.13840 .0025

0.13590 .0026

0.11990 .0024

0.12290 .0026

0.12990 .0024

0.13340 .0023 $\begin{array}{lll}0.9550 & 0.0052\end{array}$

$0.7954 \quad 0.0047$

0.90100 .0049

0.87150 .0048

0.80890 .0046

0.80520 .0047

$\begin{array}{lll}0.6226 & 0.0038\end{array}$

0.76170 .0044

$0.7000 \quad 0.0042$

0.71230 .0041 
TABLE 5.5 (contd)

\begin{tabular}{|c|c|c|c|c|c|}
\hline Matrix & Loc ${ }^{a}$ & ${ }^{235} U(n, f)^{b}$ & ${ }^{238} \mathrm{U}(n, f)^{\mathrm{b}}$ & \multicolumn{2}{|c|}{${ }^{238} U(n, \gamma)^{b}$} \\
\hline 24562 & $I J$ & $\begin{array}{lll}5.941 & 0.031\end{array}$ & $0.1423 \quad 0.0024$ & 0.7561 & 0.0044 \\
\hline 24463 & $I J$ & 5.9180 .029 & 0.13770 .0024 & 0.7408 & 0.0043 \\
\hline 24464 & IJ & 5.9560 .031 & & & \\
\hline 24465 & $I J$ & 5.7710 .030 & 0.13580 .0024 & 0.7176 & 0.0042 \\
\hline 24466 & $I J$ & 5.7090 .030 & & & \\
\hline 24367 & $I J$ & $5.344 \quad 0.029$ & $0.1268 \quad 0.0022$ & 0.7740 & 0.0044 \\
\hline 24368 & IJ & 5.0420 .027 & $\begin{array}{lll}0.1236 & 0.0024\end{array}$ & 0.7222 & 0.0045 \\
\hline 24369 & IJ & 4.4330 .026 & $0.1074 \quad 0.0024$ & 0.5621 & 0.0036 \\
\hline 24370 & $I J$ & $3.846 \quad 0.024$ & $0.0998 \quad 0.0023$ & 0.5609 & 0.0036 \\
\hline 24270 & IJ & $3.678 \quad 0.024$ & $\begin{array}{lll}0.0870 & 0.0021\end{array}$ & 0.4677 & 0.0034 \\
\hline 24273 & IJ & $\begin{array}{lll}2.717 & 0.017\end{array}$ & $\begin{array}{lll}0.0751 & 0.0020\end{array}$ & 0.3884 & 0.0030 \\
\hline 24173 & IJ & 2.8700 .018 & 0.07740 .0021 & 0.3994 & 0.0031 \\
\hline 24174 & IJ & 2.9270 .019 & $0.0788 \quad 0.0020$ & 0.4153 & 0.0029 \\
\hline 24175 & JK & 2.8060 .019 & 0.08190 .0020 & 0.3988 & 0.0028 \\
\hline 24176 & $I J$ & 2.5510 .015 & 0.06940 .0019 & 0.3630 & 0.0026 \\
\hline 24077 & $\mathrm{IJ}$ & 2.1960 .014 & $0.0528 \quad 0.0014$ & 0.3185 & 0.0026 \\
\hline 24078 & IJ & 1.8660 .015 & & & \\
\hline 24079 & IJ & 1.5320 .013 & & & \\
\hline $239 \cdot 80$ & IJ & $1.197 \quad 0.011$ & & & \\
\hline 23981 & IJ & $0.950 \quad 0.010$ & & & \\
\hline 23982 & IJ & $0.801 \quad 0.007$ & & & \\
\hline & data & the $30^{\circ} \mathrm{ra}$ & at $z=280.2$ & & \\
\hline 24654 & IJ & 5.6450 .029 & & & \\
\hline 24555 & IJ & 4.7630 .026 & & & \\
\hline 24458 & IJ & 4.4250 .024 & & & \\
\hline 24358 & IJ & $4.788 \quad 0.026$ & & & \\
\hline 24359 & IJ & $5.240 \quad 0.026$ & & & \\
\hline 24260 & IJ & $\begin{array}{lll}5.659 & 0.029\end{array}$ & & & \\
\hline 24261 & IJ & $5.980 \quad 0.030$ & & & \\
\hline 24262 & IJ & $6.096 \quad 0.030$ & & & \\
\hline 24162 & $I J$ & $6.118 \quad 0.030$ & & & \\
\hline 24065 & IJ & 5.8020 .030 & & & \\
\hline 23965 & IJ & $5.749 \quad 0.029$ & & & \\
\hline 23966 & IJ & $5.156 \quad 0.026$ & & & \\
\hline $238 \quad 67$ & $I J$ & $4.684 \quad 0.025$ & & & \\
\hline 23868 & $\mathrm{IJ}$ & $4.604 \quad 0.025$ & & & \\
\hline 23768 & $I J$ & 4.3170 .025 & & & \\
\hline & data & $n$ the $x$-axis & $z=51.6 \mathrm{~mm}^{\mathrm{C}}$ & & \\
\hline 24950 & IJ & $\begin{array}{ll}8.771 & 0.027\end{array}$ & $0.2063 \quad 0.0030$ & 1.2621 & 0.0065 \\
\hline 24951 & IJ & 8.5110 .037 & $\begin{array}{lll}0.2031 & 0.0030\end{array}$ & 1.0684 & 0.0058 \\
\hline 24952 & IJ & $8.557 \quad 0.036$ & $0.2063 \quad 0.0029$ & 1.2196 & 0.0063 \\
\hline 24953 & $I J$ & 8.2720 .036 & 0.20460 .0031 & 1.0310 & 0.0055 \\
\hline 24954 & IJ & 8.2710 .035 & 0.21220 .0030 & 1.1797 & 0.0066 \\
\hline 24955 & $I J$ & $\begin{array}{lll}7.955 & 0.038\end{array}$ & $\begin{array}{lll}0.1984 & 0.0030\end{array}$ & 1.0007 & 0.0060 \\
\hline 24956 & IJ & $7.978 \quad 0.025$ & 0.19310 .0028 & 1.1479 & 0.0066 \\
\hline
\end{tabular}


TABLE 5.5 (contd)

\begin{tabular}{|c|c|c|c|c|}
\hline Matrix & $\mathrm{Loc}^{\mathrm{a}}$ & $235 \mathrm{U}(n, f)^{\mathrm{b}}$ & ${ }^{238} U(n, f)^{b}$ & ${ }^{230} \cup(n, \gamma)^{b}$ \\
\hline 24957 & $I J$ & $\begin{array}{lll}7.792 & 0.035\end{array}$ & $0.1844 \quad 0.0027$ & 0.97840 .0053 \\
\hline 24958 & $I J$ & 7.9810 .036 & 0.19360 .0026 & $1.1367 \quad 0.0063$ \\
\hline 24959 & IJ & $8.023 \quad 0.036$ & 0.20170 .0028 & 1.13950 .0065 \\
\hline 24960 & IJ & $\begin{array}{lll}7.832 & 0.035\end{array}$ & $\begin{array}{lll}0.1951 & 0.0028\end{array}$ & 0.98160 .0058 \\
\hline 24961 & IJ & 8.0940 .038 & 0.19380 .0029 & $1.1446 \quad 0.0065$ \\
\hline 24962 & IJ & $8.094 \quad 0.037$ & $\begin{array}{lll}0.1955 & 0.0029\end{array}$ & 1.15590 .0066 \\
\hline 249.63 & IJ & 7.9390 .036 & $\begin{array}{lll}0.1886 & 0.0027\end{array}$ & 0.98560 .0063 \\
\hline 249.64 & $I J^{*}$ & & $\begin{array}{lll}0.1937 & 0.0027\end{array}$ & 1.14650 .0059 \\
\hline 24965 & IJ & $\begin{array}{lll}7.883 & 0.031\end{array}$ & 0.19050 .0029 & $1.1112 \quad 0.0063$ \\
\hline 24966 & IJ & 7.4960 .033 & $\begin{array}{lll}0.1825 & 0.0026\end{array}$ & 0.93290 .0055 \\
\hline 24967 & IJ & $\begin{array}{lll}7.393 & 0.033\end{array}$ & 0.17370 .0025 & $1.0418 \quad 0.0059$ \\
\hline 24968 & IJ & 7.0020 .032 & 0.16600 .0026 & 0.86910 .0052 \\
\hline 24969 & $I J$ & $\begin{array}{lll}6.836 & 0.031\end{array}$ & $0.1640 \quad 0.0026$ & $0.9716 \quad 0.0052$ \\
\hline 24970 & IJ & $6.417 \quad 0.029$ & 0.15270 .0026 & 0.80730 .0048 \\
\hline 24971 & IJ & $\begin{array}{lll}6.151 & 0.028\end{array}$ & $0.1518 \quad 0.0023$ & $0.8734 \quad 0.0048$ \\
\hline 24972 & $\mathrm{I} J$ & $\begin{array}{lll}5.741 & 0.026\end{array}$ & 0.14570 .00 & $0.7188 \quad 0.0042$ \\
\hline 24973 & IJ & $5.548 \quad 0.026$ & 0.14190 .00 & 900.0 \\
\hline 24974 & $\mathrm{JK}$ & $\begin{array}{lll}5.131 & 0.024\end{array}$ & $0.1404 \quad 0.0023$ & 1910.0044 \\
\hline 24976 & IJ & 4.0610 .020 & 0.10930 .0020 & $\begin{array}{ll}735 & 0.0037\end{array}$ \\
\hline 24977 & IJ & 3.5350 .019 & $0.0825 \quad 0.0019$ & 800. \\
\hline 24978 & IJ & 3.0490 .019 & 0.03660 .0012 & 7040.0 \\
\hline 24979 & $\mathrm{IJ}$ & 2.4730 .015 & 0.02010 .01 & 400.1 \\
\hline 24980 & IJ & $\begin{array}{lll}1.995 & 0.013\end{array}$ & 0.01180 .0 & 2730.0 \\
\hline 24981 & IJ & 1.6130 .011 & 0.00690 .0 & 630.0019 \\
\hline 24982 & IJ & 1.3920 .011 & 0.00430 .0008 & 4230.1 \\
\hline 24983 & $\mathrm{TC}$ & 1.4310 .011 & & \\
\hline & & 150 & $=-53$ & \\
\hline 24850 & IJ & $\begin{array}{lll}8.635 & 0.041\end{array}$ & $0.2086 \quad 0.0031$ & 1.25420 .0068 \\
\hline 24852 & $\mathrm{IJ}$ & 8.3320 .036 & 0.20290 .0029 & 4520.0 \\
\hline 24853 & IJ & 8.3290 .036 & $0.2078 \quad 0.0028$ & 1.18290 .0066 \\
\hline 24754 & IJ & $8.060 \quad 0.038$ & 0.20090 .0029 & $1.1470 \quad 0.0070$ \\
\hline 24756 & IJ & $\begin{array}{lll}7.582 & 0.033\end{array}$ & $0.1856 \quad 0.0025$ & 1.10470 .0058 \\
\hline 24757 & IJ & $7.484 \quad 0.033$ & $0.1834 \quad 0.0027$ & $1.0800 \quad 0.0061$ \\
\hline 24657 & $I J^{*}$ & & 0.16420 .0026 & 0.87220 .0048 \\
\hline 24658 & $I J$ & $\begin{array}{lll}7.131 & 0.032\end{array}$ & 0.17290 .0026 & 1.02650 .0060 \\
\hline 24659 & IJ & 7.3460 .032 & 0.17810 .0029 & $0.9288 \quad 0.0055$ \\
\hline 24660 & IJ & $7.764 \quad 0.037$ & $0.1844 \quad 0.0027$ & $1.0998 \quad 0.0062$ \\
\hline 24560 & IJ & $\begin{array}{llll}7.480 & 0.035\end{array}$ & $0.1790 \quad 0.0026$ & 0.93840 .0056 \\
\hline 24561 & $I J$ & $\begin{array}{lll}7.759 & 0.036\end{array}$ & 0.18910 .0028 & 1.09390 .0066 \\
\hline $245 \quad 62$ & IJ & 7.6370 .034 & 0.18650 .0027 & 6280.0056 \\
\hline 24463 & IJ & 7.5640 .034 & $0.1796 \quad 0.0027$ & $0.9417 \quad 0.0061$ \\
\hline 24464 & IJ & $7.675 \quad 0.034$ & 0.18130 .0028 & 1.08370 .0062 \\
\hline 24465 & $I J$ & $7.364 \quad 0.032$ & 0.17110 .0026 & $0.9150 \quad 0.0054$ \\
\hline 24466 & IJ & $\begin{array}{llll}7.317 & 0.033\end{array}$ & $\begin{array}{lll}0.1747 & 0.0027\end{array}$ & 1.04490 .0060 \\
\hline 24367 & IJ & $\begin{array}{lll}6.959 & 0.031\end{array}$ & 0.16980 .0026 & 0.98740 .0057 \\
\hline 24368 & IJ & 6.5710 .033 & $0.1607 \quad 0.0024$ & 0.94520 .0055 \\
\hline 24369 & IJ & 5.9390 .029 & 0.14650 .0028 & 0.75210 .0043 \\
\hline
\end{tabular}


TABLE 5.5 (contd)

\begin{tabular}{|c|c|c|c|c|}
\hline Matrix & $\operatorname{Loc}^{a}$ & ${ }^{235} U(n, f)^{b}$ & $238 U(n, f)^{b}$ & $2{ }^{38} U(n, y)^{b}$ \\
\hline 24370 & IJ & $\begin{array}{lll}5.317 & 0.025\end{array}$ & 0.12840 .0023 & 0.77740 .0044 \\
\hline 24270 & IJ & $5.074 \quad 0.027$ & 0.12210 .0022 & $0.6466: 0.0041$ \\
\hline 24273 & $I J$ & $3.998 \quad 0.021$ & $\begin{array}{lll}0.1058 & 0.0022\end{array}$ & $0.5790 \quad 0.0039$ \\
\hline 241.73 & IJ & $4.140 \quad 0.022$ & 0.10490 .0022 & 0.58260 .0036 \\
\hline 24174 & IJ & $\begin{array}{lll}4.087 & 0.021\end{array}$ & $0.1124 \quad 0.0023$ & 0.58220 .0036 \\
\hline 24175 & JK & 3.8390 .020 & 0.11390 .0022 & $0.5498 \quad 0.0034$ \\
\hline 24176 & IJ & 3.4340 .019 & $0.0950 \quad 0.0020$ & 0.48620 .0031 \\
\hline 240.77 & IJ & 2.8980 .016 & 0.07140 .0016 & $0.4228 \quad 0.0028$ \\
\hline 24078 & IJ & 2.4860 .016 & 0.03160 .0012 & $0.2985 \quad 0.0028$ \\
\hline 24079 & I J & 2.0570 .014 & 0.01810 .0010 & $0.2458 \quad 0.0025$ \\
\hline 23980 & IJ & $\begin{array}{lll}1.569 & 0.012\end{array}$ & 0.00950 .0009 & $0.1782 \quad 0.0018$ \\
\hline 23981 & IJ & 1.2570 .010 & $0.0060 \quad 0.0008$ & 0.13630 .0016 \\
\hline 23982 & IJ & $\begin{array}{lll}1.065 & 0.009\end{array}$ & 0.00320 .0009 & $0.1107 \quad 0.0017$ \\
\hline
\end{tabular}

data on the $30^{\circ}$ radial at $z=51.6 \mathrm{~mm}^{\mathrm{c}}$

$\begin{array}{lllllllll}246 & 54 & \text { IJ } & 7.658 & 0.035 & 0.1846 & 0.0026 & 0.9696 & 0.0052 \\ 245 & 55 & \text { IJ } & 6.755 & 0.034 & 0.1651 & 0.0027 & 0.8764 & 0.0049 \\ 244 & 58 & \text { IJ } & 6.286 & 0.031 & 0.1489 & 0.0028 & 0.8122 & 0.0047 \\ 243 & 58 & \text { IJ } & 6.736 & 0.031 & 0.1591 & 0.0028 & 0.9480 & 0.0052 \\ 243 & 59 & \text { IJ } & 7.140 & 0.032 & 0.1682 & 0.0029 & 0.9008 & 0.0050 \\ 242 & 60 & \text { IJ } & 7.430 & 0.035 & 0.1763 & 0.0029 & 0.9364 & 0.0052 \\ 242 & 61 & \text { IJ } & 7.727 & 0.034 & 0.1819 & 0.0029 & 1.1016 & 0.0058 \\ 242 & 62 & \text { IJ } & 7.766 & 0.034 & 0.1763 & 0.0029 & 1.1138 & 0.0059 \\ 241 & 62 & \text { IJ } & 7.827 & 0.036 & 0.1697 & 0.0028 & 1.1269 & 0.0059 \\ 240 & 65 & \text { IJ } & 7.361 & 0.035 & 0.1579 & 0.0026 & 0.9087 & 0.0050 \\ 239 & 65 & \text { IJ } & 7.324 & 0.036 & 0.1612 & 0.0026 & 1.0702 & 0.0057 \\ 239 & 66 & \text { IJ } & 6.710 & 0.035 & 0.1581 & 0.0030 & 0.8383 & 0.0048 \\ 238 & 67 & \text { IJ } & 6.144 & 0.031 & 0.1478 & 0.0026 & 0.7727 & 0.0048 \\ 238 & 68 & \text { IJ } & 6.039 & 0.036 & 0.1502 & 0.0027 & 0.8636 & 0.0048 \\ 237 & 68 & \text { IJ } & 5.690 & 0.031 & 0.1450 & 0.0028 & 0.7313 & 0.0047\end{array}$

data on the ysaxis at $z=51.6 \mathrm{~mm}^{\mathrm{C}}$

$\begin{array}{lllll}247 & 49 & \text { IJ } & 8.619 & 0.041 \\ 246 & 49 & \text { IJ } & 8.323 & 0.039 \\ 245 & 49 & \text { IJ } & 8.372 & 0.039 \\ 244 & 49 & \text { IJ } & 8.128 & 0.038 \\ 243 & 49 & \text { IJ } & 7.498 & 0.036 \\ 242 & 49 & \text { IJ } & 7.120 & 0.035 \\ 241 & 49 & \text { IJ } & 6.131 & 0.029 \\ 238 & 49 & \text { IJ } & 6.671 & 0.031 \\ 237 & 49 & \text { IJ } & 7.098 & 0.033 \\ 236 & 49 & \text { IJ } & 7.586 & 0.034 \\ 235 & 49 & \text { IJ } & 7.732 & 0.036 \\ 234 & 49 & \text { IJ } & 7.555 & 0.035 \\ 233 & 49 & \text { IJ } & 7.933 & 0.037 \\ 230 & 49 & \text { IJ } & 7.071 & 0.033\end{array}$


TABLE 5.5 (contd)

\begin{tabular}{|c|c|c|c|c|}
\hline Matrix & Loc $^{a}$ & $2^{23} U(n, f)^{b}$ & $2^{38} U(n, f)^{b}$ & $2^{3 \mathrm{U}} \mathrm{U}(n, \gamma)^{\mathrm{b}}$ \\
\hline $229 \quad 49$ & IJ & 6.7370 .033 & & \\
\hline $228 \quad 49$ & IJ & 6.3310 .030 & & \\
\hline $227 \quad 49$ & IJ & $6.109 \quad 0.029$ & & \\
\hline 22649 & IJ & $\begin{array}{lll}5.778 & 0.030\end{array}$ & & \\
\hline 22549 & $M N$ & 5.2730 .025 & & \\
\hline $224 \quad 49$ & $H I$ & $4.890 \quad 0.025$ & & \\
\hline 22349 & $M N$ & $4.528 \quad 0.024$ & & \\
\hline $222 \quad 49$ & $H I$ & $4.000 \quad 0.023$ & & \\
\hline $221 \quad 49$ & HI & 3.4690 .019 & & \\
\hline 22049 & $M N$ & $2.948 \quad 0.018$ & & \\
\hline 21949 & $\mathrm{HI}$ & 2.4390 .017 & & \\
\hline
\end{tabular}

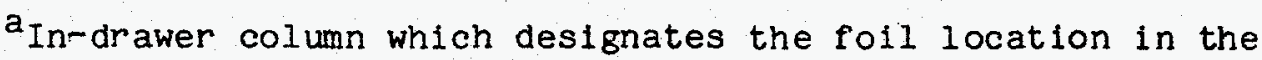
drawer. The ${ }^{235} \mathrm{U}$ foils were centered $13.8 \mathrm{~mm}$ above and the ${ }^{238} \mathrm{U}$ foils were centered on the mid-height of the drawer.

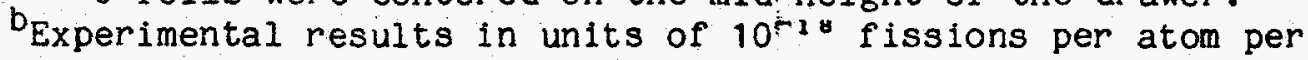
second at a reactor power of approximately one watt. The second number is one standard deviation of uncertainty. See text for detalls.

${ }^{C}$ istance from the reactor interface to the center of the foll except "*" which were at $50.3 \mathrm{~mm}$. 
TABLE 5.6 Basic Data for Radial

Distributions of ${ }^{25} U(n, f)$

in Half 1 of ZPPR-18B

\begin{tabular}{|c|c|c|c|}
\hline Matrix & $\operatorname{Loc}^{a}$ & ${ }^{235} \mathrm{U}(n, f)^{b}$ & ${ }^{235} U(n, f)^{b}$ \\
\hline -axis & ta at & $z=51.6 \mathrm{~mm}, c$ & $z=280.2 \mathrm{~mm}^{\mathrm{C}}$ \\
\hline 14950 & $\mathrm{GH}$ & $9.040 \quad 0.042$ & $7.962 \cdot 0.040$ \\
\hline 14951 & $\mathrm{GH}$ & 8.7610 .042 & $7.730 \quad 0.039$ \\
\hline 14952 & $\mathrm{GH}$ & 8.7810 .045 & $\begin{array}{lll}7.800 & 0.039\end{array}$ \\
\hline 14953 & $\mathrm{GH}$ & $\begin{array}{llll}8.593 & 0.041\end{array}$ & $\begin{array}{lll}7.631 & 0.038\end{array}$ \\
\hline 14954 & $\mathrm{GH}$ & 8.6240 .040 & $\begin{array}{lll}7.752 & 0.038\end{array}$ \\
\hline 14955 & $\mathrm{GH}$ & 8.3120 .039 & $7.491 \quad 0.039$ \\
\hline 14956 & $\mathrm{GH}$ & $8.409 \quad 0.026$ & $7.578 \quad 0.024$ \\
\hline 14957 & GH & 8.2020 .036 & $7.399 \quad 0.033$ \\
\hline 14958 & GH & $8.375 \quad 0.037$ & $\begin{array}{lll}7.528 & 0.033\end{array}$ \\
\hline 14959 & $\mathrm{GH}$ & $8.375 \quad 0.038$ & 7.4120 .034 \\
\hline 14960 & $\mathrm{GH}$ & $8.174 \quad 0.038$ & $\begin{array}{ll}51 & 0.033\end{array}$ \\
\hline 14961 & $\mathrm{GH}$ & $8.320 \quad 0.039$ & $78 \quad 0.035$ \\
\hline 14962 & $\mathrm{GH}$ & 8.2790 .038 & $7.275 \quad 0.035$ \\
\hline 14963 & $\mathrm{GH}$ & 8.0970 .039 & $7.051 \quad 0.035$ \\
\hline 14965 & $\mathrm{GH}$ & 8.0250 .026 & $50 \quad 0.023$ \\
\hline 14966 & $\mathrm{GH}$ & $7.618 \quad 0.035$ & $\begin{array}{lll}58 & 0.031\end{array}$ \\
\hline 14967 & $\mathrm{GH}$ & $\begin{array}{lll}7.567 & 0.035\end{array}$ & $10 \quad 0.030$ \\
\hline 14968 & $\mathrm{GH}$ & $7.147 \quad 0.033$ & 830.030 \\
\hline 14969 & $\mathrm{GH}$ & $\begin{array}{lll}6.977 & 0.033\end{array}$ & 6.0460 .030 \\
\hline 14970 & $\mathrm{GH}$ & $\begin{array}{lll}6.568 & 0.030\end{array}$ & 5.6550 .028 \\
\hline 14971 & $\mathrm{GH}$ & 6.3750 .031 & $\begin{array}{lll}5.481 & 0.029\end{array}$ \\
\hline 14972 & $\mathrm{GH}$ & 5.9310 .030 & $87 \quad 0.027$ \\
\hline 14973 & FG & 5.6560 .030 & 4.9170 .026 \\
\hline 14974 & $\mathrm{GH}$ & $5.256 \quad 0.029$ & 4.5550 .026 \\
\hline 14976 & $\mathrm{GH}$ & $4.184 \quad 0.024$ & $3.620 \quad 0.023$ \\
\hline 14977 & $\mathrm{GH}$ & $3.622 \quad 0.022$ & $3.145 \quad 0.020$ \\
\hline 14978 & GH & $3.071 \quad 0.017$ & 2.6410 .016 \\
\hline 14979 & GH & 2.5380 .016 & $2.204 \quad 0.015$ \\
\hline 14980 & $\mathrm{GH}$ & 2.0270 .016 & $1.725 \quad 0.013$ \\
\hline 14981 & GH & $1.662 \quad 0.011$ & $1.424 \quad 0.012$ \\
\hline 14982 & $\mathrm{GH}$ & $1.410 \quad 0.013$ & $1.231 \quad 0.012$ \\
\hline $15^{\circ} \mathrm{da}$ & & $=51.6 \mathrm{~mm}, c$ & $z=280.2 \mathrm{~mm}$ \\
\hline $148 \quad 50$ & & 80.043 & \\
\hline 14852 & $\mathrm{GH}$ & 8.6510 .043 & $7.678 \quad 0.038$ \\
\hline 14853 & GH & $8.687 \quad 0.042$ & $7.728 \quad 0.041$ \\
\hline 14754 & $\mathrm{GH}$ & 8.5350 .042 & 7.7210 .040 \\
\hline 14755 & $\mathrm{GH}$ & 8.1170 .036 & $7.493 \quad 0.034$ \\
\hline 14756 & $\mathrm{GH}$ & 8.2920 .038 & 7.6020 .034 \\
\hline 14757 & $\mathrm{GH}$ & $\begin{array}{lll}8.218 & 0.037\end{array}$ & 7.6120 .033 \\
\hline 146.58 & $\mathrm{GH}$ & $8.036 \quad 0.035$ & 7.6420 .035 \\
\hline 14659 & $\mathrm{GH}$ & 7.9120 .036 & 7.2130 .034 \\
\hline $146 \quad 60$ & $\mathrm{GH}$ & $8.120 \quad 0.038$ & \\
\hline
\end{tabular}


TABLE 5.6 (contd)

\begin{tabular}{|c|c|c|c|}
\hline Matrix & $\operatorname{Loc}^{2}$ & $235 U(n, f)^{b}$ & ${ }^{235} U(n, f)^{b}$ \\
\hline 14560 & $\mathrm{GH}$ & $\begin{array}{lll}7.931 & 0.037\end{array}$ & $7.042 \quad 0.032$ \\
\hline 14561 & GH & $8.146 \quad 0.040$ & 7.1050 .035 \\
\hline 14562 & $\mathrm{GH}$ & $\begin{array}{lll}7.932 & 0.037\end{array}$ & $6.877 \quad 0.033$ \\
\hline 14463 & GH & $\begin{array}{lll}7.816 & 0.038\end{array}$ & $6.720 \quad 0.034$ \\
\hline 14464 & $\mathrm{GH}$ & $7.793 \quad 0.037$ & $\begin{array}{lll}6.757 & 0.033\end{array}$ \\
\hline 14465 & GH & $7.488 \quad 0.034$ & $6.483 \quad 0.033$ \\
\hline 14466 & $\mathrm{GH}$ & 7.5230 .034 & $6.436 \quad 0.033$ \\
\hline 14367 & $\mathrm{GH}$ & 7.1430 .033 & $6.273 \quad 0.030$ \\
\hline 14368 & $\mathrm{GH}$ & 6.9030 .032 & 6.0640 .030 \\
\hline 143.69 & $\mathrm{GH}$ & 6.3450 .031 & $\begin{array}{lll}5.748 & 0.028\end{array}$ \\
\hline 14370 & GH & $\begin{array}{lll}6.067 & 0.031\end{array}$ & $\begin{array}{lll}5.676 & 0.029\end{array}$ \\
\hline 14270 & $\mathrm{GH}$ & $5.840 \quad 0.030$ & $5.520 \quad 0.028$ \\
\hline 14273 & GH & $\begin{array}{lll}5.037 & 0.026\end{array}$ & $4.947 \quad 0.026$ \\
\hline 14173 & GH & $5.008 \quad 0.025$ & $4.826 \quad 0.024$ \\
\hline 14174 & $\mathrm{GH}$ & $\begin{array}{lll}4.565 & 0.024\end{array}$ & 4.2590 .024 \\
\hline 14175 & FG & 4.1410 .026 & 3.7570 .023 \\
\hline 14176 & $\mathrm{GH}$ & $\begin{array}{lll}3.597 & 0.022\end{array}$ & $3.237 \quad 0.021$ \\
\hline 14077 & GH & 3.0490 .020 & $2.665 \quad 0.017$ \\
\hline 14078 & $\mathrm{GH}$ & $2.566 \quad 0.018$ & \\
\hline 14079 & $\mathrm{GH}$ & 2.0710 .013 & \\
\hline 13980 & $\mathrm{GH}$ & $1.599 \quad 0.014$ & \\
\hline 13981 & $\mathrm{GH}$ & 1.2620 .013 & \\
\hline 13982 & $\mathrm{GH}$ & 1.0910 .011 & \\
\hline
\end{tabular}

an-drawer column which designates the foil location in the drawer. The ${ }^{235} \mathrm{U}$ folls were centered $13.8 \mathrm{~mm}$ above the mid-height of the drawer.

Experimental results in units of $10^{-1}$ y fissions per atom per second at a reactor power of approximately one watt. The second number is one standard deviation uncertainty. See text for details. cDistance from the reactor interface to the center of the foll. 
TABLE 5.7 Basic Data for Axial Distributions of ${ }^{239} \mathrm{Pu}$ and ${ }^{238} \mathrm{U}$ Reaction Rates in ZPPR - 18B

\begin{tabular}{|c|c|c|c|c|c|}
\hline Matrix & $\underline{\mathrm{z}, \mathrm{mm}^{\mathrm{a}}}$ & Loc & ${ }^{239} \mathrm{Pu}(\mathrm{n}, \mathrm{f})^{\mathrm{C}}$ & ${ }^{23 \mathrm{~B}} U(\mathrm{n}, \mathrm{f})^{\mathrm{C}}$ & ${ }^{23 \mathrm{~B}} \mathrm{U}(\mathrm{n}, \gamma)^{\mathrm{C}}$ \\
\hline 24949 & 737.4 & IJ & & $\begin{array}{lll}0.0066 & 0.0011\end{array}$ & $0.3440 \quad 0.0027$ \\
\hline 24949 & 686.6 & $\mathrm{IJ}$ & & 0.01020 .0013 & $\begin{array}{lll}0.3853 & 0.0027\end{array}$ \\
\hline 24949 & 635.8 & $I J$ & & 0.01380 .0011 & $\begin{array}{lll}0.4371 & 0.0029\end{array}$ \\
\hline 24949 & 585.0 & IJ & & 0.02250 .0013 & 0.50770 .0033 \\
\hline $249 \quad 49$ & 534.2 & $I J$ & & 0.03540 .0015 & 0.57570 .0036 \\
\hline 24949 & 483.4 & $I J$ & 3.9280 .023 & $\begin{array}{lll}0.0833 & 0.0020\end{array}$ & $\begin{array}{lll}0.6322 & 0.0037\end{array}$ \\
\hline 24949 & 432.6 & IJ & 4.5320 .025 & $0.1018 \quad 0.0021$ & $0.7114 \quad 0.0040$ \\
\hline $249 \cdot 49$ & 380.5 & $I J$ & $\begin{array}{lll}5.212 & 0.028\end{array}$ & 0.12510 .0022 & 0.80630 .0049 \\
\hline 24949 & 356.4 & IJ & $5.450 \quad 0.029$ & 0.13610 .0025 & 0.84410 .0050 \\
\hline 24949 & 280.2 & IJ & $\begin{array}{lll}6.380 & 0.033\end{array}$ & $\begin{array}{lll}0.1568 & 0.0031\end{array}$ & $0.9700 \quad 0.0056$ \\
\hline 24949 & 229.4 & $I J$ & 6.9350 .034 & 0.17420 .0026 & $\begin{array}{lll}1.0521 & 0.0058\end{array}$ \\
\hline 24949 & 178.6 & IJ & $\begin{array}{lll}7.438 & 0.037\end{array}$ & $\begin{array}{lll}0.1828 & 0.0028\end{array}$ & 1.13390 .0059 \\
\hline 24949 & 127.8 & $I J$ & 7.8220 .039 & 0.19330 .0031 & 1.18690 .0062 \\
\hline 24949 & 77.0 & IJ & $\begin{array}{lll}8.175 & 0.038\end{array}$ & $0.2074 \quad 0.0029$ & 1.24110 .0075 \\
\hline 249.49 & 50.3 & IJ & 8.3210 .042 & 0.20410 .0028 & 1.25570 .0064 \\
\hline 24949 & 26.2 & I J & $8.500 \quad 0.042$ & 0.21280 .0029 & $1.2686 \quad 0.0064$ \\
\hline 14949 & 26.2 & $\mathrm{GH}$ & 8.6630 .040 & 0.21230 .0029 & 1.28990 .0065 \\
\hline 149.49 & 50.3 & $\mathrm{GH}$ & $8.527 \quad 0.041$ & 0.21450 .0029 & 1.29550 .0066 \\
\hline 14949 & 77.0 & $\mathrm{GH}$ & $\begin{array}{lll}8.582 & 0.041\end{array}$ & $0.2167 \quad 0.0029$ & 1.30370 .0066 \\
\hline 14949 & 127.8 & GH & 8.5250 .044 & 0.21210 .0028 & 1.28950 .0065 \\
\hline 14949 & 178.6 & GH & 8.2980 .042 & $0.2053 \quad 0.0029$ & $1.2568 \quad 0.0064$ \\
\hline 14949 & 229.4 & GH & 8.0190 .040 & $0.2008 \quad 0.0029$ & 1.20590 .0062 \\
\hline 14949 & 280.2 & $\mathrm{GH}$ & 7.5130 .036 & $\begin{array}{lll}0.1881 & 0.0031\end{array}$ & 1.15460 .0061 \\
\hline $149 \quad 49$ & 356.4 & $\mathrm{GH}$ & $\begin{array}{lll}6.688 & 0.033\end{array}$ & 0.16310 .0026 & $1.0324 \quad 0.0054$ \\
\hline 14949 & 380.5 & GH & $\begin{array}{lll}6.317 & 0.031\end{array}$ & 0.15330 .0028 & $0.9787 \quad 0.0057$ \\
\hline $149 \quad 49$ & 432.6 & GH & $\begin{array}{lll}5.667 & 0.030\end{array}$ & 0.13380 .0025 & 0.89140 .0049 \\
\hline 14949 & 483.4 & $\mathrm{GH}$ & $5.014 \quad 0.028$ & $\begin{array}{lll}0.1077 & 0.0023\end{array}$ & 0.80660 .0056 \\
\hline 14949 & 534.2 & $\mathrm{CH}$ & & 0.04750 .0017 & 0.75400 .0043 \\
\hline 14949 & 585.0 & GH & & 0.02840 .0012 & $0.6846 \quad 0.0039$ \\
\hline 14949 & 635.8 & GH & & $0.0177 \quad 0.0011$ & 0.60180 .0036 \\
\hline 14949 & 686.6 & GH & & 0.01140 .0010 & 0.53350 .0033 \\
\hline 149.49 & 737.4 & $\mathrm{GH}$ & & 0.00770 .0010 & 0.47630 .0033 \\
\hline 24975 & 686.6 & JK & & $\begin{array}{lll}0.0058 & 0.0009\end{array}$ & 0.17740 .0019 \\
\hline 24975 & 635.8 & JK & & $0.0090 \quad 0.0012$ & 0.20750 .0018 \\
\hline 24975 & 585.0 & JK & & 0.01320 .0011 & 0.24230 .0020 \\
\hline 24975 & 534.2 & JK & & 0.02380 .0013 & $0.2727 \quad 0.0022$ \\
\hline 24975 & 483.4 & JK & & 0.05260 .0017 & $0.3203 \quad 0.0024$ \\
\hline 24975 & 432.6 & JK & & 0.06590 .0020 & 0.35950 .0026 \\
\hline 24975 & 381.8 & JK & & $0.0807 \quad 0.0022$ & 0.40750 .0028 \\
\hline 24975 & 331.0 & JK & & $0.0949 \quad 0.0022$ & 0.46340 .0034 \\
\hline $249 \cdot 75$ & 280.2 & JK & & $\begin{array}{lll}0.1057 & 0.0025\end{array}$ & 0.50360 .0032 \\
\hline 24975 & 229.4 & JK & & 0.11410 .0025 & $0.5576 \quad 0.0035$ \\
\hline 24975 & 178.6 & JK & & $\begin{array}{lll}0.1219 & 0.0024\end{array}$ & 0.58840 .0036 \\
\hline 24975 & 127.8 & JK & & 0.12990 .0026 & 0.62130 .0038 \\
\hline
\end{tabular}


TABLE 5.7 (contd)

\begin{tabular}{|c|c|c|c|c|c|}
\hline Matrix & $\underline{\mathrm{z}, \mathrm{mm}^{\mathrm{a}}}$ & Loc $^{b}$ & $2{ }^{39} \mathrm{Pu}(n, f)^{c}$ & ${ }^{23} \mathrm{U}(n, f)^{\mathrm{C}}$ & ${ }^{2{ }^{3}} \mathrm{U}(n, \gamma)^{c}$ \\
\hline 24975 & 77.0 & $\mathrm{JK}$ & & 0.13340 .0023 & $\begin{array}{lll}0.6395 & 0.0040\end{array}$ \\
\hline 24975 & 50.3 & JK & & 0.13520 .0024 & $0.6490 \quad 0.0038$ \\
\hline 24975 & 26.2 & JK & & $\begin{array}{lll}0.1370 & 0.0022\end{array}$ & $0.6568 \quad 0.0041$ \\
\hline 14975 & 26.2 & $F G$ & & 0.14050 .0028 & 0.67620 .0040 \\
\hline 14975 & 50.3 & FG & & $\begin{array}{lll}0.1397 & 0.0027\end{array}$ & 0.67580 .0040 \\
\hline 14975 & 77.0 & FG & & $0.1419 \quad 0.0025$ & 0.67070 .0039 \\
\hline 14975 & 127.8 & $F G$ & & 0.13930 .0027 & 0.66210 .0039 \\
\hline 14975 & 178.6 & $F G$ & & $0.1328 \quad 0.0025$ & 0.64390 .0039 \\
\hline 14975 & 229.4 & FG & & $0.1274 \quad 0.0023$ & 0.61640 .0041 \\
\hline 14975 & 280.2 & $F G$ & & $0.1210 \quad 0.0024$ & $0.5798 \quad 0.0036$ \\
\hline 14975 & 331.0 & FG & & $0.1090 \quad 0.0022$ & 0.53770 .0034 \\
\hline 14975 & 381.8 & FG & & $\begin{array}{lll}0.0976 & 0.0021\end{array}$ & 0.49050 .0036 \\
\hline 14975 & 432.6 & FG & & $0.0820 \quad 0.0020$ & 0.43860 .0029 \\
\hline 14975 & 483.4 & FG & . & $0.0646 \quad 0.0016$ & $\begin{array}{lll}0.3957 & 0.0027\end{array}$ \\
\hline 149.75 & 534.2 & FG & & 0.02770 .0012 & $0.3437 \quad 0.0027$ \\
\hline 14975 & 585.0 & FG & & 0.01640 .0015 & 0.30820 .0023 \\
\hline 14975 & 635.8 & FG & & 0.01210 .0010 & $0.2705 \quad 0.0022$ \\
\hline 14975 & 686.6 & FG & & $0.0074 \quad 0.0009$ & 0.23820 .0020 \\
\hline
\end{tabular}

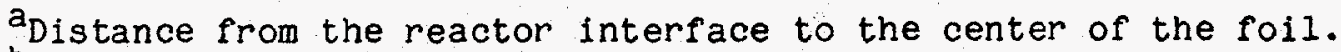

In-drawer column which designates the foll location in the drawer. The ${ }^{23 \mathrm{U}} \mathrm{U}$ foils were centered on and the ${ }^{235} \mathrm{Pu}$ folls were centered $13.8 \mathrm{~mm}$ below the mid-height of the drawer.

Experimental: results in units of $10^{-1}$ fissions or captures per atom per second at a reactor power of approximately one watt. The second number is one standard deviation uncertainty. See text for details. 
TABLE 5.8 Basic Data for Axial Distributions of ${ }^{235} \mathrm{U}(\mathrm{n}, \mathrm{f})$ in $\mathrm{ZPPR}-18 \mathrm{~B}$

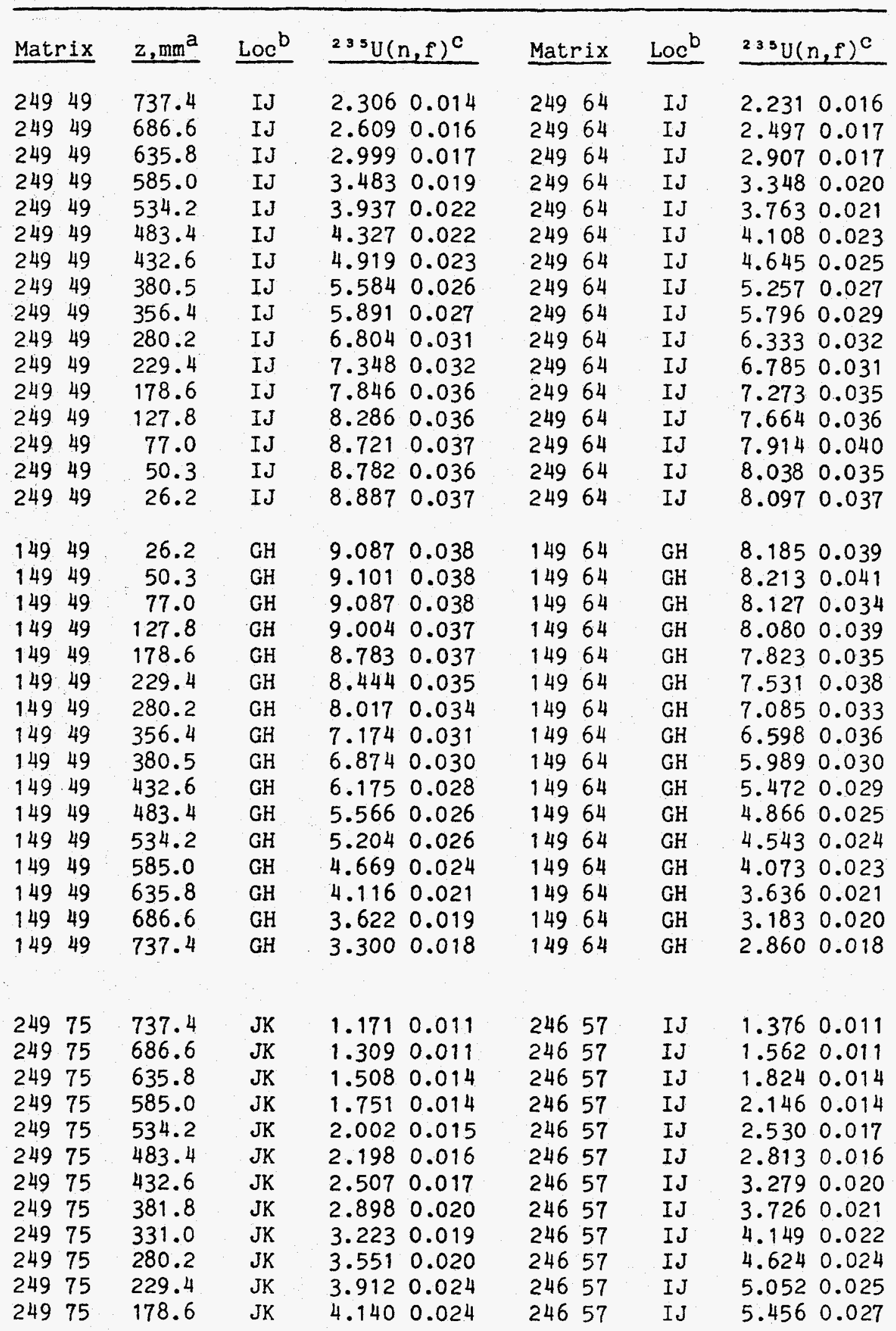


TABLE 5.8 (contd)

\begin{tabular}{|c|c|c|c|c|c|c|}
\hline Matrix & $\underline{\mathrm{z}, \mathrm{mm}^{\mathrm{a}}}$ & Loc $^{\mathrm{b}}$ & $235 U(n, f)^{c}$ & Matrix & Loc $^{b}$ & ${ }^{235} \mathrm{U}(n, f)^{\mathrm{C}}$ \\
\hline 24975 & 127.8 & JK & $4.400 \quad 0.025$ & 24657 & $I J$ & $\begin{array}{lll}5.822 & 0.028\end{array}$ \\
\hline 24975 & 77.0 & JK & $4.574 \quad 0.024$ & 24657 & IJ & $6.300 \quad 0.030$ \\
\hline 24975 & 50.3 & JK & 4.6740 .024 & 24657 & $I J$ & $6.502 \quad 0.029$ \\
\hline 24975 & 26.2 & JK & $4.700 \quad 0.023$ & $246 \quad 57$ & IJ & $6.877 \quad 0.030$ \\
\hline 14975 & 26.2 & FG & $4.734 \quad 0.027$ & $146 \quad 57$ & $\mathrm{GH}$ & 7.6910 .036 \\
\hline 14975 & 50.3 & FG & $\begin{array}{lll}4.727 & 0.026\end{array}$ & 14657 & GH & 7.9790 .038 \\
\hline 14975 & 77.0 & FG & $4.794 \quad 0.023$ & 14657 & $\mathrm{GH}$ & $\begin{array}{lll}8.155 & 0.037\end{array}$ \\
\hline 14975 & 127.8 & FG & $\begin{array}{lll}4.685 & 0.028\end{array}$ & 14657 & GH & $8.298 \quad 0.037$ \\
\hline 14975 & 178.6 & FG & $\begin{array}{lll}4.563 & 0.023\end{array}$ & 14657 & GH & $\begin{array}{lll}8.248 & 0.037\end{array}$ \\
\hline 14975 & 229.4 & FG & $4.394 \quad 0.024$ & 14657 & $\mathrm{GH}$ & $\begin{array}{lll}8.031 & 0.037\end{array}$ \\
\hline 14975 & 280.2 & FG & $4.106 \quad 0.023$ & 14657 & $\mathrm{GH}$ & 7.6710 .035 \\
\hline 14975 & 331.0 & FG & $3.768 \quad 0.021$ & 14657 & $\mathrm{GH}$ & $7.223 \quad 0.032$ \\
\hline 14975 & 381.8 & FG & $3.462 \quad 0.020$ & 14657 & $\mathrm{GH}$ & $6.670 \quad 0.032$ \\
\hline 14975 & 432.6 & FG & 3.0840 .018 & 14657 & $\mathrm{GH}$ & $6.102 \quad 0.030$ \\
\hline 14975 & 483.4 & FG & $2.720 \quad 0.017$ & 14657 & GH & $5.548 \quad 0.028$ \\
\hline 14975 & 534.2 & FG & 2.5370 .015 & 14657 & $\mathrm{GH}$ & $5.429 \quad 0.028$ \\
\hline 14975 & 585.0 & EG & $2.278 \quad 0.014$ & 14657 & $\mathrm{GH}$ & $4.968 \quad 0.026$ \\
\hline 14975 & 635.8 & FG & $2.005 \quad 0.013$ & 14657 & $\mathrm{GH}$ & $4.443 \quad 0.023$ \\
\hline 14975 & 686.6 & FG & 1.7340 .014 & 14657 & $\mathrm{GH}$ & 4.0110 .023 \\
\hline 14975 & 737.4 & $F G$ & $1.583 \quad 0.014$ & 14657 & $\mathrm{GH}$ & 3.6750 .023 \\
\hline
\end{tabular}

${ }^{a}$ Distance from the reactor interface to the center of the foll.

In-drawer column which designates the foll location in the drawer. The ${ }^{235} \mathrm{U}$ foils were centered $13.8 \mathrm{~mm}$ above the mid-height of the drawer.

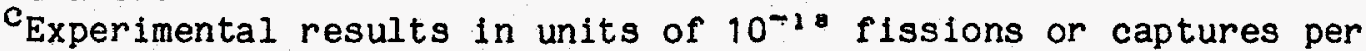
atom per second at a reactor power of approximately one watt. The second number is one standard deviation uncertainty. See text for detalis. 
6. IN-CELL REACTION RATE MEASUREMENTS AND CELL FACTORS FOR ZPPR 18 A AND ZPPR-18B (J. M. Gasidlo and D. W. Maddison)

\subsection{Introduction}

Reaction rate maps are measured by placing foils in matrix positions chosen to measure specific distributions as a radial map or an axial distribution. These "mapping" foils are always placed in the same location in a drawer for a specific type of cell. Then, cell factors are used to convert the reaction rates measured in the mapping foil to reaction rates averaged over the materials used to construct the cell.

\subsection{Description of the Int-Cell Measurements}

Cell factors were measured in special experiments where the ordinary plates used to build the reactor were replaced with special plates. Ordinary ZPPR fuel plates were replaced with two half-thickness fuel plates for which the sum of the fuel alloy is equal to one ordinary fuel plate. Foils were placed between and on the outer surfaces of the two

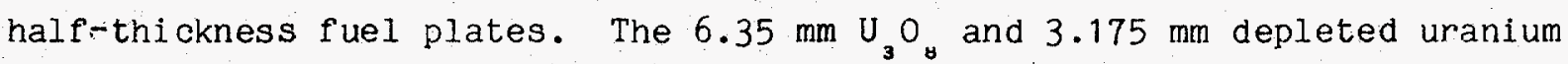
metal plates were replaced with special plates that have slots machined across the width of the plates in which special foils can be placed to measure the integral of the reaction rate across the width of the plate.

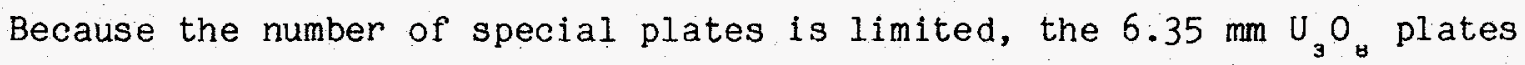

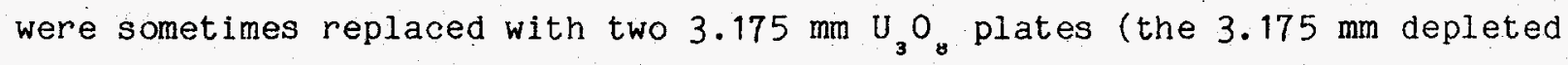
uranium metal plates with $1.588 \mathrm{~mm}$ plates) and the plate average was measured by a foil placed between the two plates.

The folls were first placed in stainless steel or aluminum holders, or in the slots of the special plates. The foil holders were then placed between the vertical columns* of materlals in the drawers. The

*ZPPR drawers are divided into sixteen $3.175 \mathrm{~mm}(1 / 8 \mathrm{in}$.) wide vertical columns designated by letters A through $P$. The letter A denotes the column at the left as one faces the drawer. The letters FG, for example, indicate the foil is between columns $F$ and $G$, and is centered $19.050 \mathrm{~mm}$ ( $0.750 \mathrm{in.})$ from the left side of the drawer. 
special plates replaced the normal plates used in the drawers. (These plates are designated by a negative sign in front of the in-drawer column location.) Foils were placed in, between or on the surface of any plates containing the foil material: ${ }^{23 y} \mathrm{Pu}$ foils are used only at fuel plates whereas ${ }^{235} \mathrm{U}$ and ${ }^{238} \mathrm{U}$ foils are used at fuel plates, depleted uranium metal plates and depleted uranium oxide plates. In all cells at least one foil of each type was placed in the "mapping foil" location.

In $2 P P R-18$ the cell studies were directed towards measuring cell factors in cells different from those in ZPPR 17 and repeating the cell studies done adjacent to control rod positions (CRPs) and control rods (CRs). Cell studies were not done in the ${ }^{235} \mathrm{U}$ fueled drawers. Measurements of cell factors in ${ }^{235} \mathrm{U}$ fueled cores indicate that the cell factor for ${ }^{235} \mathrm{U}(n, f)$ can be set equal to unity $\pm 1 \%$ for the ${ }^{235} \mathrm{U}$ fueled drawers. Cell factors for ${ }^{238} \mathrm{U}$ in the ${ }^{235} \mathrm{U}$ fueled drawers $\mathrm{w} 111$ be measured in a subsequent assembly. For all other types of cells in ZPPR 18 , the cell factors from ZPPR-17 were used without change.

For the cell studies adjacent to CRPs and CRs, half of the cell was measured on one side of the CRP (or CR) and half on the other side. Even then, there was not sufficient room in the cell to contain the necessary folls and foil holders. In that case, the column of $6.35 \mathrm{~mm} \mathrm{U}_{3} \mathrm{O}_{8}$ plates on the side of the cell away from the cell studies was replaced with a $3.18 \mathrm{~mm}$ column of $U_{3} O_{4}$ plates and a $1.59 \mathrm{~mm}$ column of alternating depleted uranium metal and stainless steel plates. This substitution made room for the cell study foils and changed the uranium content in that column by $0.2 \%$.

The basic data for cell studies in the two irradiations in ZPPR $18 \mathrm{~B}$ were combined and are given in Tables 6.1 and 6.2 . The data are given in units of $10^{\mathrm{m} / \mathrm{S}}$ fission or captures per second per atom in the irradiated foil at an estimated reactor power of one watt. The second number associated with each reaction is one standard deviation estimate of the uncertainty. As described below in section 6.4 the uncertainties given in the tables are solely due to counting statistics and data reduction. They include the effects of peak integration, interference peak corrections, 
corrections for other reactions in the foils, irradiation history and foil positioning in the counter system.

\subsection{Derivation of Cell-Averaging Factors}

The results of special experiments were used to convert the data from the special plate measurements to in-plate or plate-averaged reaction rates. These plate-averaged reaction rates were divided by calculated values to remove the effects of the gross reactor flux distribution, atomweighted plate-by-plate to determine the cell-averaged reaction rate, and divided by the mapping foil value to determine the cell factor. This process is described in greater detail in ANL-85-44.

\subsection{Discussion of Cell Factors and Uncertainties}

The cell factors for ZPPR-18A and 18B are given in Table 6.3. The second number associated with each cell factor is a one standard deviation estimate of the uncertainty. The uncertainties in the cell factors are solely due to propagation of the uncertainties in the basic data tables. They are systematic for the use of a single cell factor, but they are random when applied to the cell-averaged reaction rates for different cell factors. The listed uncertainties do not include systematic uncertainties such as deriving plate-averaged reaction rates from half-thickness plate data or other uncertainties such as those due to detector calibrations. See ANL-85-44 for a discussion of these additional uncertainties.

Under the heading "ENVIRONMENT" is listed the type of cell and cell environment for which the cell factor is applicable. The mapping foil is always located in the center drawer, the center drawer has the two indicated drawers on either side, and the mapping foil is located closer to the left-hand drawer. (See Section 5.2 on the method used for specifying the in-drawer location of mapping foils.). The key is: SF is a drawer with one fuel column that has iron oxide plates on both sides of the fuel plate, $S M$ is a drawer with one fuel column that has depleted uranium metal plates on both sides of the fuel plate, $D$ is a drawer with two fuel columns, $F$ is any fueled drawer, $A B$ is axial blanket, $R B$ is radial blanket, and $R$ is axial 
or radial reflector. The code $F / S F / R B$ means that the cell factor is used for a drawer with one fuel column that has iron oxide plates on both sides of the fuel plate with any fueled drawer on one side and a radial blanket drawer on the other with the mapping foll closer to the fueled drawer. 
TABLE 6.1. Basic Data for ${ }^{23} \mathrm{Pu}(\mathrm{n}, \mathrm{f})$ Cell Studies in ZPPR-18B

\begin{tabular}{|c|c|c|c|c|c|c|c|}
\hline Matrix & $\underline{\mathrm{z}, \mathrm{mm}^{\mathrm{a}}}$ & Loc $^{b}$ & ${ }^{2{ }^{3 y}} \mathrm{Pu}(\mathrm{n}, \mathrm{f})^{\mathrm{c}}$ & Matrix & $\underline{\mathrm{z}, \mathrm{mm}^{\mathrm{a}}}$ & $\operatorname{Loc}^{b}$ & ${ }^{2{ }^{3 y}} \mathrm{Pu}(n, f)^{\mathrm{c}}$ \\
\hline 24440 & 51.6 & $\mathrm{GH}$ & $6.054 \quad 0.033$ & 24443 & 77.0 & GH & $6.257 \quad 0.033$ \\
\hline $244 \quad 40$ & 51.6 & HI & $6.121 \quad 0.033$ & $244 \quad 43$ & 77.0 & $\mathrm{HI}$ & 6.0530 .033 \\
\hline 24440 & 51.6 & I J & 6.2920 .032 & $244 \quad 43$ & 77.0 & IJ & $\begin{array}{lll}5.993 & 0.033\end{array}$ \\
\hline 14440 & 254.8 & $\mathrm{GH}$ & $7.078 \quad 0.035$ & $144 \quad 43$ & 254.8 & $\mathrm{GH}$ & $7.473 \quad 0.040$ \\
\hline 14440 & 254.8 & HI & $7.004 \quad 0.033$ & 14443 & 254.8 & HI & 7.2770 .036 \\
\hline $144 \quad 40$ & 254.8 & $\mathrm{IJ}$ & 7.1550 .034 & $144 \quad 43$ & 254.8 & IJ & $\begin{array}{lll}7.382 & 0.037\end{array}$ \\
\hline $148 \quad 37$ & 77.0 & $\mathrm{GH}$ & $7.704 \quad 0.038$ & $148 \quad 35$ & 77.0 & $\mathrm{GH}$ & $7.769 \quad 0.038$ \\
\hline $148 \quad 37$ & 77.0 & $\mathrm{HI}$ & 7.6830 .039 & 14835 & 77.0 & $\mathrm{HI}$ & $7.656 \cdot 0.037$ \\
\hline 14837 & 77.0 & IJ & 7.6860 .039 & 14835 & 77.0 & IJ & $7.749 \quad 0.038$ \\
\hline 14837 & 254.8 & GH & 6.9430 .035 & $148 \quad 35$ & 127.8 & $\mathrm{GH}$ & 7.6890 .039 \\
\hline 14837 & 254.8 & $\mathrm{HI}$ & $6.923 \quad 0.034$ & 14835 & 127.8 & $\mathrm{HI}$ & 7.6200 .039 \\
\hline 14837 & 254.8 & IJ & $\begin{array}{lll}6.979 & 0.035\end{array}$ & $148 \quad 35$ & 127.8 & $I J$ & $7.693 \quad 0.037$ \\
\hline $150 \quad 24$ & 77.0 & $\mathrm{DE}$ & $\begin{array}{lll}4.727 & 0.026\end{array}$ & $148 \quad 35$ & 483.4 & GH & $\begin{array}{lll}4.531 & 0.027\end{array}$ \\
\hline 15024 & 77.0 & EF & 4.8180 .026 & 14835 & 483.4 & $\mathrm{HI}$ & $4.394 \quad 0.027$ \\
\hline 15024 & 77.0 & FG & $4.894 \quad 0.027$ & 14835 & 483.4 & IJ & 4.6100 .026 \\
\hline $148 \quad 24$ & 77.0 & JK & $\begin{array}{lll}4.971 & 0.027\end{array}$ & & & & \\
\hline 14824 & 77.0 & $\mathrm{KL}$ & 4.9870 .027 & & & & \\
\hline 148.24 & 77.0 & LM & $5.068 \quad 0.027$ & & & & \\
\hline
\end{tabular}

${ }^{2}$ Distance from the reactor interface to the center of the foil

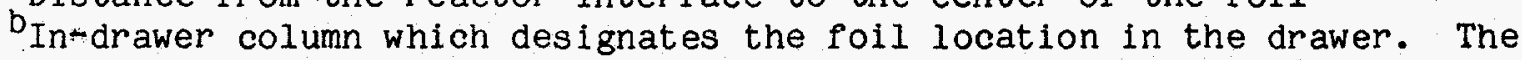
$2{ }^{3} \mathrm{Pu}$ folls were centered $13.8 \mathrm{~mm}$ below the mid-height of the drawer.

$c^{c}$ Experimental results in units of $10^{-18}$ fissions per sec per atom at $a$ reactor power of approximately one watt. The second number is one standard deviation uncertainty. See text for details. 
TABLE 6.2 Basic Data for Cell Studies of ${ }^{235} \mathrm{U}$ and ${ }^{238} \mathrm{U}$ Reaction Rates in ZPPR-18B

\begin{tabular}{|c|c|c|c|c|c|}
\hline Matrix & $\underline{\mathrm{z}, \mathrm{mm}^{\mathrm{a}}}$ & Loc ${ }^{b}$ & $235 U(n, f)^{c}$ & ${ }^{23^{8}} U(n, f)^{c}$ & ${ }^{238} \mathrm{U}(n, \gamma)^{\mathrm{C}}$ \\
\hline $254 \quad 43$ & 77.0 & $\mathrm{AB}$ & $\begin{array}{lll}6.767 & 0.030\end{array}$ & 0.13980 .0026 & 0.91910 .0055 \\
\hline 24443 & 77.0 & $-A B$ & & $0.1410 \quad 0.0029$ & $0.9368 \quad 0.0056$ \\
\hline $244 \quad 43$ & 77.0 & $\mathrm{GH}$ & $\begin{array}{lll}6.600 & 0.030\end{array}$ & 0.15160 .0029 & 0.95470 .0058 \\
\hline 24443 & 77.0 & $\mathrm{HI}$ & 6.2960 .026 & $0.1563 \quad 0.0025$ & $0.8640 \quad 0.0048$ \\
\hline $244 \quad 43$ & 77.0 & $\mathrm{IJ}$ & $6.298 \quad 0.029$ & 0.14740 .0028 & $0.9122 \quad 0.0051$ \\
\hline 24443 & 77.0 & $-O P$ & & $0.1210 \quad 0.0024$ & 0.82170 .0055 \\
\hline 254.43 & 77.0 & $O P$ & 5.8350 .025 & 0.11960 .0029 & 0.81310 .0047 \\
\hline 254.43 & 254.8 & $\mathrm{AB}$ & $\begin{array}{lll}5.251 & 0.024\end{array}$ & $0.1048 \quad 0.0024$ & 0.71130 .0052 \\
\hline $244 \quad 43$ & 254.8 & $-A B$ & & 0.11070 .0026 & 0.71530 .0045 \\
\hline $244 \quad 43$ & 254.8 & $\mathrm{GH}$ & $5.050 \quad 0.026$ & $0.1173 \quad 0.0027$ & $0.7308 \quad 0.0043$ \\
\hline $244 \quad 43$ & 254.8 & $\mathrm{HI}$ & $\begin{array}{lll}4.844 & 0.022\end{array}$ & 0.12210 .0024 & 0.65910 .0039 \\
\hline 24443 & 254.8 & IJ & $\begin{array}{llll}4.783 & 0.021\end{array}$ & 0.11110 .0024 & .68920 .0041 \\
\hline $244 \quad 43$ & 254.8 & -OP & & 0.09190 .0022 & 0.62290 .0041 \\
\hline $254 \quad 43$ & 254.8 & $\mathrm{OP}$ & $\begin{array}{lll}4.489 & 0.021\end{array}$ & 0.09530 .0023 & 0.62020 .0039 \\
\hline 15443 & 77.0 & $A B$ & $8.344 \quad 0.034$ & $0.1500 \quad 0.0030$ & $\begin{array}{ll}387 & 0.0060\end{array}$ \\
\hline 14443 & 77.0 & $-A B$ & & $0.1440 \quad 0.0028$ & 1.15710 .0064 \\
\hline 144.43 & 77.0 & $\mathrm{GH}$ & $8.240 \quad 0.033$ & $0.1750 \quad 0.0030$ & $1.2043 \quad 0.0067$ \\
\hline 14443 & 77.0 & $\mathrm{HI}$ & $8.102 \quad 0.033$ & 0.18490 .0029 & 1.09330 .0057 \\
\hline 14443 & 77.0 & $I J$ & 8.2720 .034 & $0.1782 \quad 0.0028$ & 1.18930 .0066 \\
\hline 14443 & 77.0 & $O P$ & & 0.16350 .0029 & $1.1250 \quad 0.0064$ \\
\hline $154 \quad 43$ & 77.0 & $O P$ & 8.3380 .035 & 0.16180 .0030 & 1.10810 .0063 \\
\hline $154 \quad 43$ & 254.8 & $\mathrm{AB}$ & $8.231 \quad 0.035$ & 0.13420 .0030 & 1.11050 .0059 \\
\hline $144 \quad 43$ & 254.8 & $-A B$ & & $0.1356 \quad 0.0028$ & $1.1418 \quad 0.0064$ \\
\hline 14443 & 254.8 & GH & $8.190 \quad 0.035$ & $0.1618 \quad 0.0031$ & $1.1860 \quad 0.0062$ \\
\hline $144 \quad 43$ & 254.8 & $\mathrm{HI}$ & 7.9170 .034 & $\begin{array}{lll}0.1762 & 0.0028\end{array}$ & 1.05180 .0056 \\
\hline 14443 & 254.8 & $I J$ & 8.0340 .035 & $0.1646 \quad 0.0029$ & 1.15350 .0060 \\
\hline 14443 & 254.8 & TOP & & $\begin{array}{llll}0.1504 & 0.0029\end{array}$ & 1.06830 .0067 \\
\hline 15443 & 254.8 & $O P$ & 7.9630 .033 & $\begin{array}{lll}0.1485 & 0.0029\end{array}$ & 1.04460 .0060 \\
\hline $150 \quad 35$ & 77.0 & $A B$ & $8.166 \quad 0.036$ & $0.1698 \quad 0.0024$ & 1.09300 .0060 \\
\hline 14835 & 77.0 & $-A B$ & & 0.16550 .0031 & 1.10060 .0063 \\
\hline 14835 & 77.0 & $\mathrm{GH}$ & $8.200 \quad 0.036$ & $0.1928 \quad 0.0025$ & $1.1626 \quad 0.0064$ \\
\hline 14835 & 77.0 & $\mathrm{HI}$ & 8.1060 .036 & 0.20130 .0029 & 1.08520 .0057 \\
\hline 14835 & 77.0 & $I J$ & 8.2030 .037 & 0.19170 .0026 & 1.16650 .0065 \\
\hline 14835 & 77.0 & $-O P$ & & $\begin{array}{lll}0.1705 & 0.0032\end{array}$ & $1.1128 \quad 0.0063$ \\
\hline 15035 & 77.0 & $\mathrm{OP}$ & $\begin{array}{lll}8.310 & 0.038\end{array}$ & 0.17120 .0027 & 1.10590 .0062 \\
\hline 150.35 & 127.8 & $A B$ & 8.0260 .036 & 0.16490 .0024 & 1.06910 .0056 \\
\hline 14835 & 127.8 & $-A B$ & & 0.16140 .0032 & 1.08260 .0062 \\
\hline 14835 & 127.8 & $\mathrm{GH}$ & $8.076 \quad 0.035$ & $0.1896 \quad 0.0024$ & 1.15950 .0060 \\
\hline 14835 & 127.8 & $\mathrm{HI}$ & $7.979 \quad 0.036$ & 0.20190 .0028 & 1.06450 .0056 \\
\hline 14835 & 127.8 & $I J$ & 8.0240 .035 & $0.1878 \quad 0.0026$ & $1.1651 \quad 0.0061$ \\
\hline $148 \quad 35$ & 127.8 & -OP & & $0.1644 \quad 0.0028$ & $\begin{array}{lll}1.0856 & 0.0062\end{array}$ \\
\hline $150 \quad 35$ & 127.8 & OP & 8.1910 .040 & $0.1650 \quad 0.0026$ & 1.08140 .0062 \\
\hline
\end{tabular}


TABLE 6.2 (contd)

\begin{tabular}{|c|c|c|c|c|c|}
\hline Matrix & $\underline{\mathrm{z}, \mathrm{mm}^{\mathrm{a}}}$ & Loc ${ }^{b}$ & ${ }^{235} U(n, f)^{c}$ & ${ }^{23} \mathrm{U} U(n, f)^{\mathrm{C}}$ & ${ }^{238} U(n, \gamma)^{c}$ \\
\hline 15035 & 483.4 & $\mathrm{AB}$ & 4.9330 .025 & $0.0742 \quad 0.0017$ & 0.64990 .0038 \\
\hline 14835 & 483.4 & $-A B$ & & 0.07970 .0021 & 0.66440 .0044 \\
\hline 14835 & 483.4 & $\mathrm{GH}$ & $5.073 \quad 0.025$ & $0.0907 \quad 0.0018$ & $0.7393 \quad 0.0046$ \\
\hline $148 \quad 35$ & 483.4 & $\mathrm{HI}$ & $\begin{array}{lll}5.039 & 0.025\end{array}$ & $\begin{array}{lll}0.0981 & 0.0019\end{array}$ & $0.6624 \quad 0.0038$ \\
\hline $148 \quad 35$ & 483.4 & IJ & $5.247 \quad 0.026$ & 0.09390 .0020 & 0.80710 .0046 \\
\hline $148 \cdot 35$ & 483.4 & ०OP & & $\begin{array}{llll}0.0772 & 0.0022\end{array}$ & 0.70540 .0046 \\
\hline 15035 & 483.4 & $O P$ & 5.0510 .026 & $0.0750 \quad 0.0017$ & 0.66210 .0039 \\
\hline 25440 & 51.6 & $A B$ & $\begin{array}{lll}6.043 & 0.028\end{array}$ & $0.1243 \quad 0.0022$ & 0.82150 .0049 \\
\hline 25440 & 51.6 & GG & 6.3290 .029 & 0.14240 .0023 & 0.79220 .0045 \\
\hline 24440 & 51.6 & $\mathrm{GH}$ & 6.2800 .030 & 0.14170 .0021 & 0.81030 .0049 \\
\hline 24440 & 51.6 & $\mathrm{HI}$ & 6.4120 .030 & $\begin{array}{lll}0.1577 & 0.0025\end{array}$ & 0.83480 .0046 \\
\hline 24440 & 51.6 & IJ & $\begin{array}{lll}6.561 & 0.032\end{array}$ & 0.14910 .0022 & 0.83740 .0046 \\
\hline $254 \quad 40$ & 51.6 & $\mathrm{JJ}$ & $\begin{array}{lll}6.616 & 0.031\end{array}$ & 0.14820 .0025 & $0.8368 \quad 0.0047$ \\
\hline 25440 & 51.6 & $O P$ & $6.916 \quad 0.033$ & 0.14230 .0025 & 0.92250 .0051 \\
\hline $254 \quad 40$ & 254.8 & $A B$ & $4.390 \quad 0.022$ & 0.08820 .0018 & 0.60340 .0036 \\
\hline $254 \quad 40$ & 254.8 & GG & $\begin{array}{lll}4.592 & 0.023\end{array}$ & 0.10590 .0020 & 0.59250 .0035 \\
\hline 24440 & 254.8 & GH & 4.5790 .024 & 0.11200 .0018 & 0.60170 .0035 \\
\hline $244 \quad 40$ & 254.8 & $\mathrm{HI}$ & 4.7210 .024 & 0.12230 .0021 & 0.61650 .0036 \\
\hline 24440 & 254.8 & IJ & $4.865 \quad 0.024$ & $\begin{array}{lll}0.1128 & 0.0022\end{array}$ & 0.62850 .0038 \\
\hline $254 \cdot 40$ & 254.8 & JJ & 4.9230 .026 & $\begin{array}{lll}0.1120 & 0.0021\end{array}$ & $0.6244 \quad 0.0037$ \\
\hline $254 \quad 40$ & 254.8 & $O P$ & 5.2010 .025 & 0.10440 .0019 & 0.69870 .0044 \\
\hline 15440 & 51.6 & $A B$ & $8.008 \quad 0.034$ & $\begin{array}{lll}0.1523 & 0.0022\end{array}$ & $\begin{array}{lll}1.0427 & 0.0058\end{array}$ \\
\hline 15440 & 51.6 & GG & 7.8090 .033 & 0.16240 .0025 & 0.96180 .0056 \\
\hline 14440 & 51.6 & $\mathrm{GH}$ & $\begin{array}{lll}7.672 & 0.032\end{array}$ & 0.17310 .0023 & $0.9694 \quad 0.0054$ \\
\hline 14440 & 51.6 & HI & $7.722 \quad 0.032$ & $0.1790 \quad 0.0025$ & 0.99040 .0056 \\
\hline 14440 & 51.6 & IJ & $\begin{array}{lll}7.816 & 0.033\end{array}$ & $0.1647 \quad 0.0023$ & 0.97770 .0055 \\
\hline 15440 & 51.6 & $\mathrm{JJ}$ & $7.793 \quad 0.033$ & $0.1591 \quad 0.0022$ & 0.96690 .0054 \\
\hline 15440 & 51.6 & $\mathrm{OP}$ & $\begin{array}{lll}7.971 & 0.034\end{array}$ & $\begin{array}{lll}0.1383 & 0.0022\end{array}$ & 1.07560 .0061 \\
\hline 15440 & 254.8 & $A B$ & $\begin{array}{l}7.773 \quad 0.033 \\
\end{array}$ & 0.14210 .0023 & 1.00980. \\
\hline $154 \quad 40$ & 254.8 & GG & $\begin{array}{lll}7.679 & 0.033\end{array}$ & 0.15290 .0022 & $0.9254 \cdot 0.0058$ \\
\hline 14440 & 254.8 & $\mathrm{GH}$ & $\begin{array}{lll}7.540 & 0.031\end{array}$ & $0.1554 \quad 0.0023$ & 0.94580 .0058 \\
\hline 14440 & 254.8 & HI & $\begin{array}{ll}7.561 & 0.032\end{array}$ & 0.16660 .0024 & 0.95790 .0054 \\
\hline 14440 & 254.8 & IJ & $\begin{array}{lll}7.719 & 0.033\end{array}$ & $0.1543 \quad 0.0021$ & $0.9520 \quad 0.0057$ \\
\hline 15440 & 254.8 & $\mathrm{JJ}$ & $\begin{array}{lll}7.750 & 0.033\end{array}$ & $\begin{array}{lll}0.1472 & 0.0021\end{array}$ & 0.94360 .0054 \\
\hline 15440 & 254.8 & $\mathrm{OP}$ & $\begin{array}{lll}5.792 & 0.025\end{array}$ & $0.1306 \quad 0.0021$ & $1.0896 \quad 0.0060$ \\
\hline 15037 & 77.0 & $\mathrm{AB}$ & $8.295 \quad 0.034$ & 0.16930 .0029 & 1.09780 .0062 \\
\hline 150 & 77.0 & GG & 8.0950 .033 & $0.1886 \quad 0.0038$ & $1.0130 \quad 0.0055$ \\
\hline 14837 & 77.0 & $\mathrm{GH}$ & 8.0580 .032 & 0.19430 .0028 & $\begin{array}{lll}1.0183 & 0.0058\end{array}$ \\
\hline $148 \quad 37$ & 77.0 & $\mathrm{HI}$ & 8.0210 .034 & 0.20290 .0030 & 1.03280 .0055 \\
\hline $148 \quad 37$ & 77.0 & IJ & 8.0300 .034 & 0.19340 .0027 & 1.01890 .0053 \\
\hline 15037 & 77.0 & J J & 8.1130 .034 & $\begin{array}{lll}0.1828 & 0.0028\end{array}$ & 1.00910 .0054 \\
\hline 15037 & 77.0 & OP & 8.2630 .035 & 0.17420 .0032 & 1.09070 .0059 \\
\hline
\end{tabular}


TABLE 6.2 (contd)

\begin{tabular}{|c|c|c|c|c|c|}
\hline Matrix & $\underline{\mathrm{z}, \mathrm{mm}^{\mathrm{a}}}$ & Loc $b$ & ${ }^{235} U(n, f)^{c}$ & ${ }^{2{ }^{8}} \mathrm{U}(\mathrm{n}, \mathrm{f})^{\mathrm{C}}$ & $2^{28} U(n, Y)^{c}$ \\
\hline 15037 & 254.8 & $A B$ & $7.475 \quad 0.031$ & 0.14620 .0027 & 0.98030 .0053 \\
\hline 150.37 & 254.8 & GG & 7.3010 .030 & 0.16390 .0029 & $0.9116 \quad 0.0051$ \\
\hline $148 \quad 37$ & 254.8 & $\mathrm{GH}$ & $7.273 \quad 0.033$ & $0.1705 \quad 0.0026$ & 0.91450 .0054 \\
\hline $148 \cdot 37$ & 254.8 & HI & $7.266 \quad 0.031$ & 0.18630 .0027 & 0.92630 .0050 \\
\hline $148 \quad 37$ & 254.8 & IJ & 7.2740 .031 & 0.17290 .0027 & $0.9248 \quad 0.0054$ \\
\hline $150 \quad 37$ & 254.8 & $\mathrm{JJ}$ & $\begin{array}{lll}7.301 & 0.031\end{array}$ & 0.16650 .0031 & $0.9210 \quad 0.0051$ \\
\hline 15037 & 254.8 & $\mathrm{OP}$ & $7.486 \quad 0.031$ & 0.14850 .0030 & 0.98920 .0054 \\
\hline 15024 & 77.0 & $\mathrm{AB}$ & $4.975 \quad 0.024$ & 0.12830 .0021 & 0.63640 .0044 \\
\hline $150 \quad 24$ & 77.0 & DE & 4.9790 .023 & $0.1427 \quad 0.0021$ & $0.6618 \quad 0.0041$ \\
\hline 15024 & 77.0 & $E F$ & 4.9740 .023 & 0.15320 .0022 & 0.65210 .0042 \\
\hline 15024 & 77.0 & FG & 5.0810 .023 & 0.15130 .0022 & 0.71880 .0044 \\
\hline 14824 & 77.0 & $\mathrm{JK}$ & $5.124 \quad 0.022$ & $0.1484 \quad 0.0024$ & 0.72710 .0044 \\
\hline $148 \quad 24$ & 77.0 & $\mathrm{KL}$ & $5.134 \quad 0.025$ & $0.1557 \quad 0.0021$ & $0.6787 \quad 0.0042$ \\
\hline 14824 & 77.0 & LM & 5.1790 .024 & 0.14740 .0021 & 0.70420 .0048 \\
\hline 14824 & 77.0 & OP & $5.294 \quad 0.024$ & 0.13210 .0020 & 0.69870 .0043 \\
\hline 150.24 & 483.4 & $\mathrm{AB}$ & 2.8660 .015 & 0.05760 .0014 & 0.37130 .0031 \\
\hline 15024 & 483.4 & $\mathrm{DE}$ & $2.876 \quad 0.016$ & 0.06550 .0015 & 0.39190 .0029 \\
\hline 15024 & 483.4 & EF & $2.854 \quad 0.015$ & 0.07240 .0014 & $0.3720 \quad 0.0035$ \\
\hline $150 \quad 24$ & 483.4 & FG & $2.956 \quad 0.015$ & $0.0676 \quad 0.0014$ & 0.43180 .0030 \\
\hline $148 \quad 24$ & 483.4 & $\mathrm{JK}$ & 3.0150 .016 & 0.06970 .0016 & 0.43950 .0030 \\
\hline 14824 & 483.4 & $\mathrm{KL}$ & 2.9960 .016 & 0.07160 .0016 & $0.3902 \quad 0.0032$ \\
\hline 14824 & 483.4 & LM & $\begin{array}{lll}3.062 & 0.017\end{array}$ & 0.06870 .0014 & 0.42160 .0030 \\
\hline 14824 & 483.4 & $\mathrm{OP}$ & $3.109 \quad 0.017$ & 0.06140 .0014 & 0.40610 .0030 \\
\hline
\end{tabular}

$a_{D i s t a n c e}$ from the reactor interface to the center of the foil.

b In-drawer column which designates the foil location in the drawer. The ${ }^{235} \mathrm{U}$ foils were centered $13.8 \mathrm{~mm}$ above and the ${ }^{238} \mathrm{U}$ foils were centered on the mid-height of the drawer. A negative sign designates a plate-spanning averaging foil.

Experimental results in units of $10^{\text {-1 }}$ fissions or captures per atom per second at a reactor power of approximately one watt. The second number is one standard deviation uncertainty. See text for details. 
TABLE 6.3 Cell-Averaging Factors for ZPPR-18A and ZPPR -18B

\begin{tabular}{|c|c|c|c|c|c|c|c|c|c|}
\hline \multicolumn{2}{|c|}{${ }^{2{ }^{3}{ }^{y}} \mathrm{Pu}(\mathrm{n}, \mathrm{f})^{\mathrm{a}}$} & \multicolumn{2}{|c|}{${ }^{235} \mathrm{U}(\mathrm{n}, \mathrm{f})^{\mathrm{a}}$} & \multicolumn{2}{|c|}{$2{ }^{3} \mathrm{U} U(\mathrm{n}, \mathrm{f})^{\mathrm{a}}$} & \multicolumn{2}{|c|}{${ }^{2{ }^{8} \mathrm{U}} \mathrm{U}(\mathrm{n}, \gamma)^{\mathrm{a}}$} & \multirow{2}{*}{$\frac{\text { Environment }}{F-S F+F}$} & \multirow{2}{*}{$\frac{\mathrm{z}, \mathrm{mm}^{\mathrm{c}}}{0-381.8}$} \\
\hline 0.9954 & .0058 & 1.0011 & .0049 & 0.953 & .011 & 0.9256 & .0088 & & \\
\hline 0.9918 & .0064 & 0.9950 & .0052 & 0.949 & .014 & 0.9089 & .0079 & $F \sim S F-F$ & 432.6 \\
\hline 0.9881 & .0069 & 0.9889 & .0055 & 0.945 & .016 & 0.8922 & .0069 & $F-S F=F$ & 483.4 \\
\hline 0.9960 & .0058 & 0.9983 & .0047 & 0.961 & .013 & 0.9293 & .0059 & $F-S F-C R P$ & $0-381.8$ \\
\hline 0.9839 & .0060 & 0.9922 & .0089 & 0.965 & .014 & 0.9118 & .0086 & $C R P-S F \sim F$ & $0 \div 381.8$ \\
\hline 0.9801 & .0060 & 0.9760 & .0051 & 0.966 & .016 & 0.9170 & .0063 & $F-S F-C R$ & $0-381.8$ \\
\hline 1.0066 & .0063 & 1.0072 & .0058 & 0.978 & .016 & 0.9490 & .0065 & $\mathrm{CR}-\mathrm{SF}-\mathrm{F}$ & $0-381.8$ \\
\hline 0.9850 & .0059 & 1.0020 & .0057 & 0.978 & .019 & 0.9020 & .0051 & $\mathrm{~F}-\mathrm{SF}-\mathrm{RB}$ & $0-381.8$ \\
\hline 0.9983 & .0041 & 1.0082 & .0046 & 0.954 & .008 & 1.0161 & .0045 & $F-S M-F$ & $0-483.4$ \\
\hline 0.9967 & .0058 & 1.0200 & .0047 & 0.972 & .016 & 1.0244 & .0066 & $F-S M-C R P$ & $0-483.4$ \\
\hline 0.9876 & .0055 & 1.0006 & .0047 & 0.969 & .011 & 1.0181 & .0065 & $C R P-S M-F$ & $0-483.4$ \\
\hline 0.9826 & .0060 & 0.9904 & .0053 & 0.977 & .018 & 1.0120 & .0096 & $F-S M-C R$ & $0-483.4$ \\
\hline 1.0077 & .0064 & 1.0255 & .0055 & 0.957 & .018 & 1.0349 & .0125 & $C R-S M-F$ & $0-483.4$ \\
\hline 0.9958 & .0060 & 1.0003 & .0049 & 0.981 & .016 & 0.9090 & .0063 & $F-D-F$ & $0-381.8$ \\
\hline 0.9917 & .0073 & 0.9971 & .0053 & 0.984 & .017 & 0.8892 & .0067 & $E-D^{4}-F$ & 432.6 \\
\hline 1.0 & .01 & 0.9939 & .0057 & 0.988 & .017 & 0.8694 & .0071 & $F-D-F$ & 483.4 \\
\hline 1.0 & .01 & 1.0022 & .0063 & 0.912 & .026 & 1.0229 & .0058 & $F \div R B-R B$ & ALL \\
\hline 1.0 & .01 & 0.9949 & .0074 & 0.988 & .071 & 1.0170 & .0071 & $\mathrm{RB}-\mathrm{RB} \sim \mathrm{RB}$ & ALL \\
\hline 1.0 & .01 & 0.9791 & .0085 & 0.838 & .100 & 1.0508 & .0090 & $R B-R B-R F$ & ALL \\
\hline 1.0 & .01 & 1.0 & .01 & 1.0 & .01 & 1.0 & .01 & $\mathrm{RR}$ & ALL \\
\hline 1.0 & .008 & 1.0005 & .0059 & 1.081 & .031 & 0.8815 & .0061 & $A B-A B=A B \quad(S C)$ & 534.2 \\
\hline 1.0 & .008 & 1.0005 & .0059 & 1.081 & .053 & 0.8521 & .0064 & $A B-A B-A B \quad(S C)$ & 585.0 \\
\hline 1.0 & .008 & 1.0005 & .0070 & 1.081 & .077 & 0.8340 & .0060 & $A B-A B-A B \quad(S C)$ & 635.8 \\
\hline 1.0 & .008 & 1.0005 & .0073 & 1.081 & .16 & 0.8053 & .0058 & $A B-A B-A B \quad(S C)$ & 686.6 \\
\hline 1.0 & .008 & 1.0005 & .0074 & 1.081 & .14 & 0.7817 & .0064 & $A B-A B=A B \quad(S C)$ & 734.4 \\
\hline 1.0 & .008 & 0.9986 & .0059 & 1.064 & .052 & 0.8627 & .0057 & $A B-A B-A B \quad(D C)$ & 534.2 \\
\hline 1.0 & .008 & 1.0005 & .0059 & 1.081 & .053 & 0.8361 & .0064 & $A B-A B-A B \quad(D C)$ & 585.0 \\
\hline 1.0 & .008 & 1.0005 & .0070 & 1.081 & .077 & 0.8340 & .0060 & $A B-A B-A B \quad(D C)$ & 635.8 \\
\hline 1.0 & .008 & 1.0005 & .0073 & 1.081 & .16 & 0.8053 & .0058 & $A B-A B-A B \quad(D C)$ & 686.6 \\
\hline 1.0 & .008 & 1.0005 & .0074 & 1.081 & .14 & 0.7817 & .0064 & $A B-A B-A B \quad(D C)$ & 734.4 \\
\hline 1.0 & .008 & 0.9947 & .0055 & 1.012 & .019 & 0.8410 & .0045 & $A B-A B-C R P \quad(S C)$ & 534.2 \\
\hline 1.0 & .008 & 0.9947 & .0055 & 1.047 & .038 & 0.8145 & .0040 & $A B-A B-C R P \quad(S C)$ & 585.0 \\
\hline 1.0 & .008 & 0.9947 & .0055 & 1.047 & .042 & 0.7839 & .0061 & $A B-A B-C R P \quad(S C)$ & 635.8 \\
\hline 1.0 & .008 & 0.9947 & .0055 & 1.047 & .063 & 0.7551 & .0052 & $A B-A B-C R P \quad(S C)$ & 686.6 \\
\hline 1.0 & .008 & 0.9947 & .0055 & 1.047 & .101 & 0.7262 & .0042 & $A B-A B-C R P \quad(S C)$ & 737.4 \\
\hline
\end{tabular}


TABLE 6.3 (contd)

\begin{tabular}{|c|c|c|c|c|c|c|c|c|c|}
\hline \multicolumn{2}{|c|}{$2{ }^{39} \mathrm{Pu}(\mathrm{n}, \mathrm{f})^{\mathrm{a}}$} & \multicolumn{2}{|c|}{${ }^{235} U(n, f)^{a}$} & \multicolumn{2}{|c|}{${ }^{2{ }^{8}} \mathrm{U}(\mathrm{n}, \mathrm{f})^{\mathrm{a}}$} & \multicolumn{2}{|c|}{$2^{38} U(n, \gamma)^{a}$} & Environment $t^{b}$ & $\mathrm{z}, \mathrm{mm}^{\mathrm{c}}$ \\
\hline 1.0 & .008 & 0.9964 & .0085 & 0.967 & .034 & 0.9241 & .0212 & $A B-A B-C R \quad(S C)$ & 534.2 \\
\hline 1.0 & .008 & 0.9964 & .0085 & 0.967 & .052 & 0.8945 & .0278 & $A B-A B-C R \quad(S C)$ & 585.0 \\
\hline 1.0 & .008 & 0.9964 & .0085 & 0.967 & .067 & 0.8868 & .0280 & $A B \sim A B-C R \quad(S C)$ & 635.8 \\
\hline 1.0 & .008 & 0.9964 & .0085 & 0.967 & .097 & 0.8539 & .0230 & $A B-A B \sim C R \quad(S C)$ & 686.6 \\
\hline 1.0 & .008 & 0.9964 & .0085 & 0.967 & .100 & 0.8377 & .0253 & $A B-A B-C R \quad(S C)$ & 737.4 \\
\hline
\end{tabular}

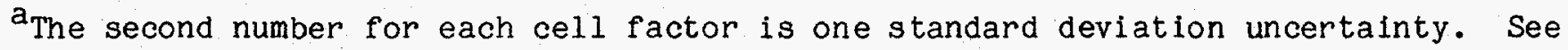
text for details.

Type of cell and local environment. See text for key and discussion.

Axial position to which this cell factor applies. 
7. GAMMA RAY DOSE MEASUREMENTS IN ZPPR -18A AND ZPPR+18B (D. N. Olsen)

Gamma ray doses were measured in $Z P P R-18 \mathrm{~A}$ and $\mathrm{ZPPR}-18 \mathrm{~B}$ as a function of axial and radial location using stainlesststeel encapsulated thermolumi nescent dosimeters (TLDs). The LIF TLDs were $1 \times 1 \times 6 \mathrm{~mm}$ and were placed in holes in stainless steel cylinders. The TLDs are somewhat sensitive to neutron interactions in the LIF. No calculations were made for gamma dose in ZPPR- 18 .

The incell location of the TLDs varied among the drawer types. The TLDs spanned two drawer columns.* For all single-fuel-column drawers and all blanket drawers, the TLDs were in columns $E$ and $F$. In doubletfuelcolumn drawers, the location was columns $C$ and $D$. In the $C R$ and $C R P$ drawers, the location was columns $G$ and $H$. For the axial distributions in ZPPR 18B, the TLDs were in columns $E$ and $F$ in half 2 and in columns $K$ and $L$ in half 1 .

Two radial distributions were measured in ZPPR-18A. The first was along the $x$-axis $76 \mathrm{~mm}$ above the axial midplane and the results are given in Table 7.1. The second was approximately $45^{\circ}$ to the x-axis with detours to go through inner, middle and outer bank control positions. Results from the second radial distribution are given in Table 7.2 .

A single axial dose distribution was measured in an inner-core location (145-63) in ZPPR-18A. This location is four drawers removed from the nearest control position. The drawer is a single-fuel-column drawer with iron oxide. Results are given in Table 7.3.

Many of the gamma dose distribution measurements were repeated in ZPPR 18B which had half-inserted control rods in the inner and outer banks. In core 18B, all radial distributions were measured $76 \mathrm{~mm}$ from the

\footnotetext{
*ZPPR drawers are divided into sixteen $3.175 \mathrm{~mm}(1 / 8 \mathrm{in}$.) wide vertical columns designated by letters A through $P$. The letter A denotes the column at the left as one faces the drawer.
} 
interface in half two, which contained the inserted rods. Results from an $x$-axis distribution are given in Table 7.4. Results from a second radial distribution going through an innerming control rod, a middle-ring CRP and an outer-ring control rod are given in Table 7.5 .

Axial distributions in ZPPR-18B were measured in both assembly halves. One location was $145-35$ and 245-35, symmetrically equivalent to measurement location 145-63 in core 18A. A second location measured in ZPPR-18B was 1,247-42 which was one drawer removed from an inner-ring CR/CRP channel. Axial results from $Z P P R-18 B$ are given in Table 7.6 .

Intra-cell variations in dose were also measured in ZPPR-18A. Results are given in Fig. 7.1. These results indicate the extent of in-cell variations and may be used to test calculations of "cell-average factors". 
TABLE 7.1 Measured Dose Rates along the x-axis in $\mathrm{ZPPR}-18 \mathrm{~A}$

\begin{tabular}{|c|c|c|c|}
\hline $\begin{array}{c}\text { Matrix } \\
\text { Location }\end{array}$ & $\begin{array}{l}\text { Drawer } \\
\text { Type }^{\mathbf{a}} \\
\end{array}$ & $\begin{array}{c}\text { Dose Rate, } \\
\mathrm{mr} \mathrm{d} / \mathrm{s}\end{array}$ & Zone \\
\hline $149-49$ & SF & 0.3716 & Inner Core \\
\hline $149-50$ & SF & 0.3768 & Inner Core \\
\hline $149-51$ & SM & 0.3360 & Inner Core \\
\hline $149-52$ & SF & 0.3685 & Inner Core \\
\hline $149-53$ & SM & 0.3271 & Inner Core \\
\hline $149 \div 54$ & SF & 0.3489 & Inner Core \\
\hline $149-55$ & SM & 0.3286 & Inner Core \\
\hline $149 \div 56$ & SF & 0.3484 & Inner Core \\
\hline $149-57$ & SM & 0.3172 & Inner Core \\
\hline $149-58$ & SF & 0.3512 & Inner Core \\
\hline $149-59$ & SF & 0.3551 & Inner Core \\
\hline $149-60$ & SM & 0.3134 & Inner Core \\
\hline $149 m 61$ & SF & 0.3417 & Inner Core \\
\hline $149-62$ & SF & 0.3478 & Inner Core \\
\hline $149-63$ & SM & 0.3000 & Inner Core \\
\hline $149-64$ & SF & 0.3376 & Inner Core \\
\hline $149-65$ & SF & 0.3315 & Inner Core \\
\hline $149-66$ & SM & 0.2892 & Inner Core \\
\hline $149-67$ & SF & 0.3219 & Inner core \\
\hline $149-68$ & SM & 0.2750 & Inner Core \\
\hline $149-69$ & SF & 0.2810 & Inner Core \\
\hline $149 \div 70$ & SM & 0.2468 & Inner Core \\
\hline $149-71$ & $S F$ & 0.2570 & Inner Core \\
\hline $149 \div 72$ & SM & 0.2254 & Inner Core \\
\hline $149-73$ & SF & 0.2482 & Outer Core \\
\hline $149-74$ & SF & 0.2508 & Outer Core \\
\hline $149-75$ & DF & 0.2611 & Outer Core \\
\hline $149-76$ & SF & 0.2000 & Outer Core \\
\hline $149-77$ & SF & 0.1542 & Outer Core \\
\hline $149-78$ & B & 0.0782 & Blanket \\
\hline $148-80$ & B & 0.0202 & Blanket \\
\hline $149-82$ & B & 0.0199 & Blanket \\
\hline
\end{tabular}

$a_{S F}$ is singlenfuel column with iron oxide, SM is singlemfuel column with depleted-uranium metal, DF is double fuel column and $B$ is blanket.

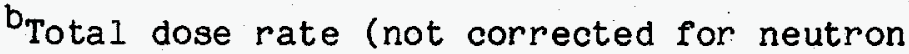
contribution) at an estimated reactor power of 1 watt. 
TABLE 7.2 Measured Dose Rates $45^{\circ}$ to the $x$-axis and through Control Positions in ZPPR 18A

\begin{tabular}{|c|c|c|c|c|}
\hline $\begin{array}{c}\text { Matrix } \\
\text { Location } \\
\end{array}$ & $\begin{array}{r}\text { Drawer } \\
\text { Type } \\
\end{array}$ & $\begin{array}{c}\text { Dose Rate, } \\
\mathrm{mrd} / \mathrm{s} \\
\end{array}$ & \multicolumn{2}{|c|}{ Zone } \\
\hline $148-50$ & $S F$ & 0.3621 & Inner & Core \\
\hline $147-51$ & $\mathrm{SF}$ & 0.3721 & Inner & Core \\
\hline $146-52$ & SF & 0.3637 & Inner & Core \\
\hline $145-53$ & $\mathrm{SF}$ & 0.3578 & Inner & Core \\
\hline $144-54$ & $\mathrm{SF}$ & 0.3470 & Inner & Core \\
\hline $144-55$ & SF & 0.3420 & Inner & Core \\
\hline $144-56$ & CRP & 0.2456 & CRP & \\
\hline $144-57$ & CRP & 0.2481 & CRP & \\
\hline $144-58$ & SM & 0.2855 & Inner & Core \\
\hline $144: 59$ & $S F$ & 0.3368 & Inner & Core \\
\hline $143-60$ & $\mathrm{SF}$ & 0.3310 & Inner & Core \\
\hline $142-61$ & $\mathrm{SF}$ & 0.3262 & Inner & Core \\
\hline $141-62$ & $\mathrm{SF}$ & 0.3025 & Inner & Core \\
\hline $140-63$ & CRP & 0.2096 & CRP & \\
\hline $140-64$ & CRP & 0.1980 & CRP & \\
\hline $140-65$ & SM & 0.2375 & Inner & Core \\
\hline $141-66$ & SM & 0.2525 & Inner & Core \\
\hline $142-67$ & $\mathrm{SM}$ & 0.2472 & Inner & Core \\
\hline $142-68$ & $S E$ & 0.2714 & Inner & Core \\
\hline $142-69$ & $S F$ & 0.2671 & Inner & Core \\
\hline $142-70$ & $\mathrm{SM}$ & 0.2238 & Inner & Core \\
\hline $142-71$ & CRP & 0.1692 & CRP & \\
\hline $142-72$ & CRP & 0.1666 & CRP & \\
\hline $142-73$ & $S F$ & 0.2107 & Outer & Core \\
\hline $142-76$ & DF & 0.3808 & Outer & Core \\
\hline $142-77$ & SF & 0.1396 & Outer & Core \\
\hline
\end{tabular}

$a_{S F}$ is single-fuel column with iron oxide, SM is single-fuel column with depleted-uranium metal, DF is double fuel column and CRP is control position.

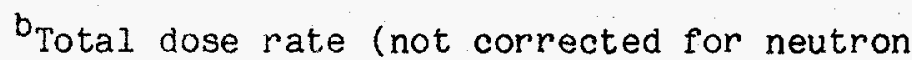
contribution) at an estimated reactor power of 1 watt. 
TABLE 7.3 Measured Axial Dose Distribution in Location $145-63$ in ZPPR-18A

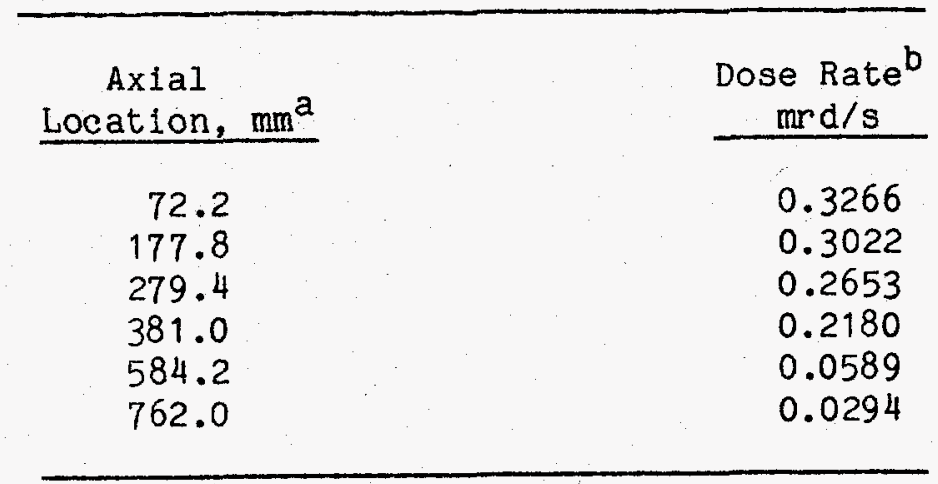

$a_{D i s t a n c e}$ from the reactor interface.

Dotal dose rate (not corrected for neutron contribution) at an estimated reactor power of 1 watt. 
TABLE 7.4 Measured Dose Rates along the $x$ axis in $\mathrm{ZPPR}-18 \mathrm{~B}$

\begin{tabular}{|c|c|c|c|}
\hline $\begin{array}{c}\text { Matrix } \\
\text { Location } \\
\end{array}$ & $\begin{array}{l}\text { Drawer } \\
\text { Type } \\
\end{array}$ & $\begin{array}{c}\text { Dose Rate, } \\
\mathrm{mrd} / \mathrm{s} \\
\end{array}$ & Zone \\
\hline $249-49$ & SF & 0.3940 & Inner Core \\
\hline $249-45$ & SM & 0.3623 & Inner Core \\
\hline $249 \div 41$ & SM & 0.3338 & Inner Core \\
\hline $249-31$ & SF & 0.3739 & Inner Core \\
\hline $249-33$ & SF & 0.3702 & Inner Core \\
\hline $249-29$ & SF & 0.3078 & Inner Core \\
\hline $249-26$ & SM & 0.2438 & Inner Core \\
\hline $249:-25$ & SF & 0.2634 & Outer Core \\
\hline $249-23$ & DF & 0.2743 & Outer Core \\
\hline $249-21$ & $\mathrm{SF}$ & 0.1573 & Outer Core \\
\hline $249-20$ & B. & 0.0893 & Blanket \\
\hline $249-17$ & B & 0.0292 & Blanket \\
\hline $249-16$ & B & 0.0212 & Blanket \\
\hline $249-15$ & $\mathrm{R}$ & 0.0134 & Reflector \\
\hline
\end{tabular}

${ }^{a} S F$ is single-fuel column with iron oxide, $S M$ is single-fuel column with depleted-uranium metal, DF is double fuel column and $B$ is blanket and $\mathrm{R}$ is reflector.

botal dose rate (not corrected for neutron contribution) at an estimated reactor power of 1 watt. 
TABLE 7.5 Measured Dose Rates through Control Rods and Control Rod Positions in ZPPR-18B

\begin{tabular}{|c|c|c|c|}
\hline $\begin{array}{l}\text { Matrix } \\
\text { Location }\end{array}$ & $\begin{array}{l}\text { Drawer } \\
\text { Type }\end{array}$ & $\begin{array}{c}\text { Dose Rate, } \\
\mathrm{mrd} / \mathrm{s}\end{array}$ & Zone \\
\hline $247-47$ & SF & 0.4008 & Inner Core \\
\hline $245-43$ & SM & 0.2808 & Inner Core \\
\hline $244-43$ & SF & 0.2847 & Inner Core \\
\hline $244-42$ & $\mathrm{CR}$ & 0.2462 & $\mathrm{CR}$ \\
\hline $244-41$ & $\mathrm{CR}$ & 0.2433 & $\mathrm{CR}$ \\
\hline $244 \div 40$ & SM & 0.2474 & Inner Core \\
\hline $243-39$ & SM & 0.3006 & Inner Core \\
\hline $243-38$ & SF & 0.3461 & Inner Core \\
\hline $242-37$ & $\mathrm{SF}$ & 0.3636 & Inner Core \\
\hline $241-36$ & SF & 0.3308 & Inner Core \\
\hline $240-35$ & CRP & 0.2425 & CRP \\
\hline $240-34$ & CRP & 0.2441 & CRP \\
\hline $240-33$ & SM & 0.2688 & Inner Core \\
\hline $243-30$ & SF & 0.3146 & Inner Core \\
\hline $243-29$ & SM & 0.2680 & Inner Core \\
\hline $242-28$ & SM & 0.2202 & Inner Core \\
\hline $242-27$ & $C R$ & 0.1823 & $\mathrm{CR}$ \\
\hline $242-26$ & $\mathrm{CR}$ & 0.1740 & $\mathrm{CR}$ \\
\hline $242-25$ & SF & 0.1834 & Outer core \\
\hline $241-25$ & SF & 0.1954 & Outer Core \\
\hline $241-24$ & SF & 0.1980 & Outer Core \\
\hline $241-22$ & SF & 0.1783 & Outer Core \\
\hline
\end{tabular}

$a_{S F}$ is single-fuel column with iron oxide, SM is singlefuel column with depleted-uranium metal, $\mathrm{CR}$ is control rod and CRP is control position.

${ }^{b}$ Total dose rate (not corrected for neutron contribution) at an estimated reactor power of 1 watt. 
TABLE 7.6 Measured Axial Dose D1stributions in $\mathrm{ZPPR}-18 \mathrm{~B}$

\begin{tabular}{|c|c|c|}
\hline \multirow[t]{2}{*}{$\begin{array}{c}\text { Axial } \\
\text { Location, } \mathrm{mm}^{\mathrm{a}} \\
\end{array}$} & \multicolumn{2}{|c|}{ Dose Rate $\mathrm{mrd} / \mathrm{s}$} \\
\hline & $1,247-42$ & $1,245-35$ \\
\hline-762.0 & 0.0245 & 0.0285 \\
\hline-584.2 & 0.0552 & 0.0651 \\
\hline-482.6 & 0.1427 & 0.1578 \\
\hline-381.0 & 0.2050 & 0.2220 \\
\hline-279.4 & 0.2607 & 0.2802 \\
\hline-177.8 & 0.2942 & 0.3242 \\
\hline-76.2 & 0.3458 & 0.3506 \\
\hline 76.2 & 0.3862 & 0.3737 \\
\hline 177.8 & 0.3618 & 0.3556 \\
\hline 279.4 & 0.3476 & 0.3122 \\
\hline 381.0 & 0.2924 & 0.2534 \\
\hline 482.6 & 0.2075 & 0.1881 \\
\hline 584.2 & 0.0877 & 0.0758 \\
\hline 762.0 & 0.0401 & 0.0387 \\
\hline
\end{tabular}

$a_{\text {With respect to zero at the reactor }}$ interface, negative values in half two.

$b_{\text {Total dose rate (not corrected for neutron }}$ contribution) at an estimated power of 1 watt. 

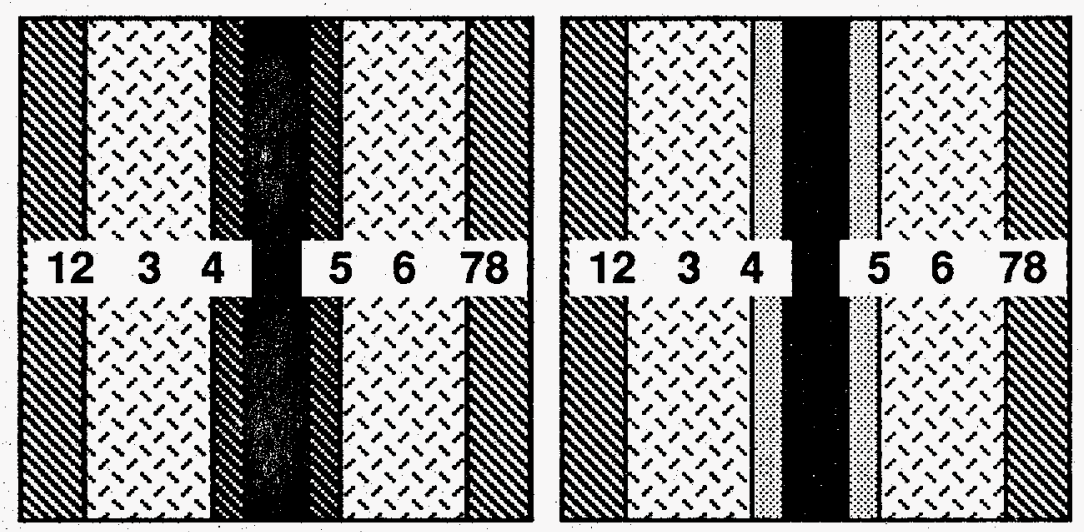

U U308

洨 Sodium

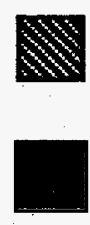

$238 \mathrm{U}$

ZPPR Fuel

$\mathrm{Fe} 2 \mathrm{O} 3$

INTRA-CELL $\quad \mathbf{2 4 9 - 5 3}$

249-52

LOCATION

DOSE RATE, $\mathrm{mrd} / \mathrm{s}$

\begin{tabular}{lll}
\hline 1 & 0.392 & 0.398 \\
2 & 0.396 & 0.422 \\
3 & 0.380 & 0.428 \\
4 & 0.384 & 0.434 \\
5 & 0.397 & 0.446 \\
6 & 0.378 & 0.416 \\
7 & 0.394 & 0.411 \\
8 & 0.391 & 0.408
\end{tabular}

Fig. 7.1 Measured In-cell Gamma Dose Distributions in ZPPR-18A 
8. CALCULATION MODELS FOR ZPPR-19A (G. L. Grasseschi and P. J. Collins)

ZPPR - 19A was a variant of the ZPPR 18 series of cores designed to reduce the fundamental to first harmonic eigenvalue separation from about $4 \% \Delta k$ to about $2 \% \Delta k$. The zone boundaries remained the same as in $Z P P R-18$, with distinct sectors in the outer core with uranium fuel and with plutonium fuel. The core was made near critical with the inner bank of six control rods fully inserted. The core required less fuel than did ZPPR-18C which had 18 control rods half-inserted. A detailed description of ZPPR-19A is given in ANL-ZPR-489, p. 2. No new drawer masters were required for ZPPR -19 .

Calculations for ZPPR-19A used the cross section library generated for $Z P P R-18$ (ANL-ZPR-489, p. 12). The reactor model used $x y z$ geometry with detailed compositions of each drawer master. The $x y$ plan is given in Fig. 8.1. The different drawer types are described in ANL-ZPR-489, Tables 4.1 and 5.1 .

Average compositions of each zone in ZPPR-19A are given in Table 8.1. 
Table 8.1 Atom Densities by Zone and Drawer Type in ZPPR-19A

\begin{tabular}{|c|c|c|c|c|c|c|}
\hline Isotope & $\begin{array}{l}\text { Inner } \\
\text { Core } \\
\text { Average } \\
0-20\end{array}$ & $\begin{array}{c}\text { Axial } \\
\text { Blanket } \\
(I C) \\
20-31 \\
\end{array}$ & $\begin{array}{l}\text { Reflector } \\
\text { Iron Block } \\
\text { (IC) } \\
31-36 \\
\end{array}$ & $\begin{array}{c}\text { Inner } \\
\text { Core } \\
(I C S F) \\
0-20 \\
\end{array}$ & $\begin{array}{c}\text { Inner } \\
\text { Core } \\
\text { (ICSM) } \\
0-20 \\
\end{array}$ & $\begin{array}{c}\text { Inner } \\
\text { Core } \\
\text { Single Pu } \\
0-20 \\
\end{array}$ \\
\hline C & 0.0001439 & 0.0000532 & 0.0005894 & 0.0000332 & 0.0000333 & 0.0000332 \\
\hline 0 & 0.0120392 & 0.0088207 & 0.0 & 0.0137453 & 0.0088143 & 0.0118548 \\
\hline $\mathrm{Na}$ & 0.0092004 & 0.0091883 & 0.0 & 0.0092715 & 0.0091481 & 0.0092241 \\
\hline Si & 0.0001588 & 0.0001924 & 0.0001264 & 0.0001570 & 0.0001587 & 0.0001577 \\
\hline Al & 0.0000041 & 0.0000029 & 0.0 & 0.0000040 & 0.0000040 & 0.0000040 \\
\hline Mn & 0.0002305 & 0.0003420 & 0.0006847 & 0.0002287 & 0.0002289 & 0.0002288 \\
\hline $\mathrm{Cr}$ & 0.0026943 & 0.0041649 & 0.0021198 & 0.0026684 & 0.0026773 & 0.0026718 \\
\hline $\mathrm{Fe}$ & 0.0115495 & 0.0147985 & 0.0759668 & 0.0128536 & 0.0095173 & 0.0115745 \\
\hline $\mathrm{Ni}$ & 0.0011851 & 0.0017648 & 0.0008612 & 0.0011678 & 0.0011814 & 0.0011730 \\
\hline $\mathrm{Cu}$ & 0.0000304 & 0.0000440 & 0.0000271 & 0.0000293 & 0.0000313 & 0.0000301 \\
\hline Mo & 0.0002503 & 0.0000346 & 0.0000136 & 0.0002397 & 0.0002383 & 0.0002392 \\
\hline U4 & 0.0 & 0.0 & $\quad 0.0$ & 0.0 & 0.0 & 0.0 \\
\hline U5 & 0.0000169 & 0.0000179 & 0.0 & 0.0000127 & 0.0000234 & 0.0000168 \\
\hline u6 & 0.0 & 0.0 & 0.0 & 0.0 & 0.0 & 0.0 \\
\hline U8 & 0.0077009 & 0.0081559 & 0.0 & 0.0058158 & 0.0106485 & 0.0076686 \\
\hline P8 & 0.0000005 & 0.0 & 0.0 & 0.0000004 & 0.0000005 & 0.0000004 \\
\hline P9 & 0.0009274 & 0.0 & 0.0 & 0.0008878 & 0.0008791 & 0.0008844 \\
\hline PO & 0.0001227 & 0.0 & 0.0 & 0.0001176 & 0.0001161 & 0.0001170 \\
\hline $\mathrm{P} 1$ & 0.0000071 & 0.0 & 0.0 & 0.0000065 & 0.0000069 & 0.0000067 \\
\hline P2 & 0.0000019 & 0.0 & 0.0 & 0.0000017 & 0019 & 0.0000018 \\
\hline A1 & 0.0000113 & 0.0 & 0.0 & 0.0000108 & 0.0000107 & 0.0000108 \\
\hline $\mathrm{P}$ & 0.0000052 & 0.0000102 & 0.0000238 & 0.0000053 & 0.0000051 & 0.0000052 \\
\hline$S$ & 0.0000011 & 0.0000081 & 0.0000314 & 0.0000010 & 0.0000011 & 0.0000010 \\
\hline $\mathrm{Cl}$ & 0.0000006 & 0.0000003 & 0.0 & 0.0000003 & 0.0000003 & 0.0000003 \\
\hline $\mathrm{Ca}$ & 0.0000020 & 0.0000021 & 0.0 & 0.0000021 & 0.0000021 & 0.0000021 \\
\hline Co & 0.0000016 & 0.0000016 & 0.0000009 & 0.0000008 & 0.0000024 & 0.0000014 \\
\hline
\end{tabular}


TABLE 8.1 (contd)

\begin{tabular}{|c|c|c|c|c|c|c|}
\hline Isotope & 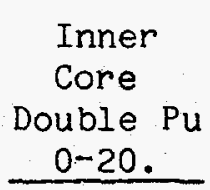 & $\begin{array}{l}\text { Outer } \\
\text { Core } \\
\text { Average } \\
0-20 . \\
\end{array}$ & $\begin{array}{c}\text { Outer } \\
\text { Core } \\
\text { Average } \\
20-20.44 \\
\end{array}$ & $\begin{array}{c}\text { Axial } \\
\text { Blanket } \\
\text { (OC) } \\
20.44-31 \\
\end{array}$ & $\begin{array}{l}\text { Reflector } \\
\text { Iron Block } \\
\text { (OC) } \\
31-36 . \\
\end{array}$ & $\begin{array}{c}\text { Outer } \\
\text { Core } \\
\text { Pu Fuel } \\
0-20 . \\
\end{array}$ \\
\hline $\mathrm{C}$ & 0.0023102 & 0.0016072 & 0.0000430 & 0.0000550 & 0.0005923 & 0.0008296 \\
\hline 0 & 0.0156589 & 0.0157892 & 0.0088279 & 0.0088211 & 0.0 & 0.0144145 \\
\hline $\mathrm{Na}$ & 0.0087410 & 0.0087498 & 0.0093061 & 0.0092564 & 0.0 & 0.0091166 \\
\hline $\mathrm{Si}$ & 0.0001814 & 0.0001676 & 0.0001702 & 0.0002039 & 0.0001167 & 0.0001656 \\
\hline Al & 0.0000061 & 0.0000044 & 0.0000028 & 0.0000029 & 0.0 & 0.0000048 \\
\hline$M n$ & 0.0002646 & 0.0002394 & 0.0002669 & 0.0003458 & 0.0006737 & 0.0002411 \\
\hline $\mathrm{Cr}$ & 0.0031355 & 0.0028416 & 0.0031949 & 0.0040979 & 0.0019566 & 0.0028304 \\
\hline $\mathrm{Fe}$ & 0.0110721 & 0.0143516 & 0.0113707 & 0.0145844 & 0.0760551 & 0.0122272 \\
\hline $\mathrm{Ni}$ & 0.0014220 & 0.0012562 & 0.0013686 & 0.0017587 & 0.0007933 & 0.0012551 \\
\hline $\mathrm{Cu}$ & 0.0000364 & 0.0000318 & 0.0000362 & 0.0000405 & 0.0000279 & 0.0000316 \\
\hline Mo & 0.0004677 & 0.0001438 & 0.0000233 & 0.0000289 & 0.0000140 & 0.0003193 \\
\hline U4 & 0.0 & 0.0000093 & 0.0000077 & 0.0 & 0.0 & 0.0 \\
\hline U5 & 0.0000183 & 0.0009624 & 0.0008004 & 0.0000179 & 0.0 & 0.0000146 \\
\hline U6 & 0.0 & 0.0000044 & 0.0000037 & 0.0 & 0.0 & 0.0 \\
\hline U8 & 0.0083377 & 0.0068596 & 0.0082019 & 0.0081562 & 0.0 & 0.0066889 \\
\hline $\mathrm{P} 8$ & 0.0000010 & 0.0000003 & 0.0 & 0.0 & 0.0 & 0.0000007 \\
\hline P9 & 0.0017696 & 0.0005021 & 0.0 & 0.0 & 0.0 & 0.0011920 \\
\hline PO & 0.0002341 & 0.0000752 & 0.0 & 0.0 & 0.0 & 0.0001786 \\
\hline P1 & 0.0000141 & 0.0000048 & 0.0 & 0.0 & 0.0 & 0.0000113 \\
\hline P2 & 0.0000039 & 0.0000017 & 0.0 & 0.0 & 0.0 & 0.0000041 \\
\hline A1 & 0.0000217 & 0.0000076 & 0.0 & 0.0 & 0.0 & 0.0000180 \\
\hline$P$ & 0.0000046 & 0.0000052 & 0.0000073 & 0.0000102 & 0.0000233 & 0.0000051 \\
\hline$S$ & 0.0000016 & 0.0000015 & 0.0000039 & 0.0000063 & 0.0000314 & 0.0000012 \\
\hline $\mathrm{Cl}$ & 0.0000062 & 0.0000043 & 0.0000003 & 0.0000003 & 0.0 & 0.0000024 \\
\hline $\mathrm{Ca}$ & 0.0000010 & 0.0000013 & 0.0000021 & 0.0000021 & 0.0 & 0.0000017 \\
\hline Co & 0.0000045 & 0.0000025 & 0.0000025 & 0.0000023 & 0.0000017 & 0.0000019 \\
\hline
\end{tabular}


TABLE 8.1 (contd)

\begin{tabular}{|c|c|c|c|c|c|c|}
\hline Isotope & $\begin{array}{c}\text { Axial } \\
\text { Blanket } \\
\text { (OC) Pu Fuel } \\
20-31 \\
\end{array}$ & $\begin{array}{c}\text { Reflector } \\
\text { Iron Block } \\
\text { (OC) Pu Fuel } \\
31-36 \\
\end{array}$ & $\begin{array}{c}\text { Outer } \\
\text { Core } \\
\text { (OC) Pu Fuel } \\
0-20 \\
\end{array}$ & $\begin{array}{c}\text { Outer } \\
\text { Core } \\
\text { Single } \mathrm{Pu} \\
0-20 \\
\end{array}$ & $\begin{array}{l}\text { Outer } \\
\text { Core } \\
\text { U Fuel } \\
0-20 \\
\end{array}$ & $\begin{array}{l}\text { Outer } \\
\text { Core } \\
\text { U Fuel } \\
20-20.44 \\
\end{array}$ \\
\hline C & 0.0000531 & 0.0005928 & 0.0023796 & 0.0000332 & 0.0012578 & 0.0000199 \\
\hline 0 & 0.0088210 & 0.0 & 0.0161289 & 0.0137451 & 0.0097183 & 0.0051099 \\
\hline $\mathrm{Na}$ & 0.0092557 & 0.0 & 0.0090421 & 0.0092985 & 0.0049102 & 0.0053839 \\
\hline Si & 0.0001926 & 0.00011 .65 & 0.0001869 & 0.0001570 & 0.0000979 & 0.0000890 \\
\hline AI & 0.0000029 & 0.0 & 0.0000061 & 0.0000041 & 0.0000024 & 0.0000017 \\
\hline $\mathrm{Mn}$ & 0.0003418 & 0.0006744 & 0.0002729 & 0.0002283 & 0.0001378 & 0.0001228 \\
\hline $\mathrm{Cr}$ & 0.0041641 & 0.0019578 & 0.0032323 & 0.0026649 & 0.0016495 & 0.0014400 \\
\hline $\mathrm{Fe}$ & 0.0147923 & 0.0761458 & 0.0114182 & 0.0128413 & 0.0092018 & 0.0051367 \\
\hline $\mathrm{Ni}$ & 0.0017667 & 0.0007914 & 0.0014630 & 0.0011662 & 0.0007276 & 0.0006244 \\
\hline $\mathrm{Cu}$ & 0.0000444 & 0.0000274 & 0.0000369 & 0.0000293 & 0.0000185 & 0.0000175 \\
\hline Mo & 0.0000348 & 0.0000138 & 0.0004825 & 0.0002391 & 0.0000093 & 0.0000086 \\
\hline U4 & 0.0 & 0.0 & 0.0 & 0.0 & 0.0000093 & 0.0000077 \\
\hline U5 & 0.0000179 & 0.0 & 0.0000187 & 0.0000127 & 0.0009563 & 0.0007928 \\
\hline U6 & 0.0 & 0.0 & 0.0 & 0.0 & 0.0000044 & 0.0000037 \\
\hline U8 & 0.0081561 & 0.0 & 0.0085400 & 0.0058281 & 0.0040424 & 0.0047667 \\
\hline P8 & 0.0 & 0.0 & 0.0000012 & 0.0000005 & 0.0 & 0.0 \\
\hline P9 & 0.0 & 0.0 & 0.0018152 & 0.0008855 & 0.0 & 0.0 \\
\hline PO & 0.0 & 0.0 & 0.0002998 & 0.0001172 & 0.0 & 0.0 \\
\hline P1 & 0.0 & 0.0 & 0.0000195 & 0.0000071 & 0.0 & 0.0 \\
\hline P2 & 0.0 & 0.0 & 0.0000084 & 0.0000019 & 0.0 & 0.0 \\
\hline A1 & $\quad 0.0$ & 0.0 & 0.0000317 & 0.0000110 & 0.0 & 0.0 \\
\hline $\mathrm{P}$ & 0.0000101 & 0.0000234 & 0.0000049 & 0.0000053 & 0.0000030 & 0.0000031 \\
\hline$S$ & 0.0000081 & 0.0000314 & 0.0000016 & 0.0000010 & 0.0000010 & 0.0000007 \\
\hline $\mathrm{Cl}$ & 0.0000003 & $\begin{array}{r}0.0 \\
0\end{array}$ & 0.0000064 & 0.0000003 & 0.0000034 & 0.0000002 \\
\hline $\mathrm{Ca}$ & 0.0000021 & 0.0 & 0.0000010 & 0.0000021 & 0.0000006 & 0.0000012 \\
\hline Co & 0.0000019 & 0.0000013 & 0.0000041 & 0.0000008 & 0.0000017 & 0.0000017 \\
\hline
\end{tabular}


TABLE 8.1 (contd)

\begin{tabular}{|c|c|c|c|c|c|c|}
\hline Isotope & $\begin{array}{c}\text { Axial } \\
\text { Blanket } \\
\text { (OC) U Fuel } \\
20.44-31 \\
\end{array}$ & $\begin{array}{c}\text { Reflector } \\
\text { Iron Block } \\
(O C) \text { U Fuel } \\
31-36 \\
\end{array}$ & $\begin{array}{c}\text { Outer Core } \\
\text { Single U } \\
\text { (oCUS) } \\
0-20 \\
\end{array}$ & $\begin{array}{c}\text { Outer Core } \\
\text { Single U } \\
\text { (OCUS) } \\
20-20.44 \\
\end{array}$ & $\begin{array}{c}\text { Outer Core } \\
\text { Double U } \\
\text { (OCUD) } \\
0-20 \\
\end{array}$ & $\begin{array}{c}\text { Outer Core } \\
\text { Double U } \\
\text { (OCUD) } \\
20-20.44 \\
\end{array}$ \\
\hline $\mathrm{C}$ & 0.0000326 & 0.0003426 & 0.0021064 & 0.0000340 & 0.0022470 & 0.0000349 \\
\hline 0 & 0.0051059 & 0.0 & 0.0171001 & 0.0088278 & 0.0164429 & 0.0088278 \\
\hline $\mathrm{Na}$ & 0.0053581 & 0.0 & 0.0083649 & 0.0092908 & 0.0086142 & 0.0093129 \\
\hline $\mathrm{Si}$ & 0.0001228 & 0.0000676 & 0.0001684 & 0.0001481 & 0.0001700 & 0.0001602 \\
\hline A1 & 0.0000017 & 0.0 & 0.0000043 & 0.0000029 & 0.0000038 & 0.0000028 \\
\hline Mn & 0.0002018 & 0.0003896 & 0.0002412 & 0.0002105 & 0.0002346 & 0.0002141 \\
\hline $\mathrm{Cr}$ & 0.0023441 & 0.0011320 & 0.0028881 & 0.0024576 & 0.0028069 & 0.0025214 \\
\hline $\mathrm{Fe}$ & 0.0083543 & 0.0439848 & 0.0177817 & 0.0087813 & 0.0137983 & 0.0089775 \\
\hline $\mathrm{Ni}$ & 0.0010146 & 0.0004600 & 0.0012690 & 0.0010563 & 0.0012435 & 0.0011037 \\
\hline $\mathrm{Cu}$ & 0.0000218 & 0.0000164 & 0.0000302 & 0.0000281 & 0.0000340 & 0.0000324 \\
\hline Mo & 0.0000142 & 0.0000082 & 0.0000147 & 0.0000133 & 0.0000175 & 0.0000167 \\
\hline U4 & 0.0 & 0.0 & 0.0000109 & 0.0000082 & 0.0000217 & 0.0000189 \\
\hline U5 & 0.0000104 & 0.0 & 0.0011314 & 0.0008546 & 0.0022319 & 0.0019431 \\
\hline U6 & 0.0 & 0.0 & 0.0000052 & 0.0000039 & 0.0000105 & 0.0000090 \\
\hline U8 & 0.0047211 & 0.0 & 0.0073446 & 0.0082050 & 0.006581 .5 & 0.0082681 \\
\hline P8 & $\quad 0.0$ & 0.0 & 0.0 & 0.0 & 0.0 & 0.0 \\
\hline P9 & 0.0 & 0.0 & 0.0 & 0.0 & 0.0 & 0.0 \\
\hline PO & 0.0 & 0.0 & 0.0 & 0.0 & 0.0 & 0.0 \\
\hline P1 & 0.0 & 0.0 & 0.0 & 0.0 & 0.0 & 0.0 \\
\hline P2 & 0.0 & 0.0 & 0.0 & 0.0 & 0.0 & 0.0 \\
\hline A1 & 0.0 & 0.0 & 0.0 & 0.0 & 0.0 & 0.0 \\
\hline $\mathrm{P}$ & 0.0000059 & 0.0000135 & 0.0000054 & 0.0000054 & 0.0000051 & 0.0000052 \\
\hline$S$ & 0.0000029 & 0.0000182 & 0.0000015 & 0.0000011 & 0.0000018 & 0.0000015 \\
\hline $\mathrm{Cl}$ & 0.0000002 & 0.0 & 0.0000056 & 0.0000003 & 0.0000060 & 0.0000003 \\
\hline $\mathrm{Ca}$ & 0.0000012 & 0.0 & 0.0000010 & 0.0000021 & 0.0000010 & 0.0000021 \\
\hline Co & 0.0000015 & 0.0000012 & 0.0000010 & 0.0000010 & 0.0000050 & 0.0000049 \\
\hline
\end{tabular}


TABLE 8.1 (contd)

\begin{tabular}{|c|c|c|c|c|c|c|}
\hline Isotope & $\begin{array}{c}\text { Radial } \\
\text { Blanket } \\
0-20\end{array}$ & $\begin{array}{c}\text { Radial } \\
\text { Blanket } \\
20-31 \\
\end{array}$ & $\begin{array}{c}\text { Radial } \\
\text { Blanket } \\
31-36\end{array}$ & $\begin{array}{c}\text { Radial } \\
\text { Reflector } \\
0-36 \\
\end{array}$ & $\begin{array}{c}\text { Axial } \\
\text { Reflector } \\
36-42 \\
\end{array}$ & $\begin{array}{c}\text { Empty } \\
\text { Matrix } \\
0-42 \\
\end{array}$ \\
\hline $\mathrm{C}$ & 0.0000318 & 0.0000319 & 0.0005928 & 0.0002493 & 0.0002143 & 0.0000188 \\
\hline 0 & 0.0193179 & 0.0193406 & 0.0 & 0.0 & 0.0 & 0.0 \\
\hline $\mathrm{Na}$ & 0.0036444 & 0.0036359 & 0.0 & 0.0 & 0.0 & 0.0 \\
\hline Si & 0.0001352 & 0.0001355 & 0.0001163 & 0.0008675 & 0.0008629 & 0.0000683 \\
\hline Al & 0.0000021 & 0.0000021 & 0.0 & 0.0 & $\quad 0.0$ & 0.0 \\
\hline Mn & 0.0001937 & 0.0001941 & 0.0006746 & 0.0014119 & 0.0015241 & 0.0001059 \\
\hline $\mathrm{Cr}$ & 0.0022430 & 0.0022455 & 0.0019586 & 0.0153499 & 0.0150441 & 0.0011891 \\
\hline $\mathrm{Fe}$ & 0.0080226 & 0.0080330 & 0.0761527 & 0.0563344 & 0.0531084 & 0.0042791 \\
\hline $\mathrm{Ni}$ & 0.0009584 & 0.0009588 & 0.0007902 & 0.0066625 & 0.0066621 & 0.0004802 \\
\hline $\mathrm{Cu}$ & 0.0000279 & 0.0000280 & 0.0000271 & 0.0000386 & 0.0000172 & 0.0000172 \\
\hline Mo & 0.0000132 & 0.0000131 & 0.0000136 & 0.0000322 & 0.0000083 & 0.0000083 \\
\hline U4 & 0.0 & 0.0 & $\begin{array}{r}0.0 \\
0\end{array}$ & $\begin{array}{r}0.0 \\
0\end{array}$ & $\begin{array}{r}0.0 \\
0\end{array}$ & 0.0 \\
\hline U5 & 0.0000369 & 0.0000370 & 0.0 & 0.0 & 0.0 & 0.0 \\
\hline U6 & 0.0 & 0.0 & 0.0 & 0.0 & 0.0 & 0.0 \\
\hline U8 & 0.0168314 & 0.0168695 & 0.0 & 0.0 & 0.0 & 0.0 \\
\hline P8 & 0.0 & 0.0 & 0.0 & 0.0 & 0.0 & 0.0 \\
\hline P9 & 0.0 & 0.0 & 0.0 & 0.0 & 0.0 & 0.0 \\
\hline PO & 0.0 & 0.0 & 0.0 & 0.0 & 0.0 & 0.0 \\
\hline P1 & 0.0 & 0.0 & 0.0 & 0.0 & 0.0 & 0.0 \\
\hline P2 & 0.0 & 0.0 & 0.0 & 0.0 & 0.0 & 0.0 \\
\hline A1 & 0.0 & 0.0 & 0.0 & 0.0 & 0.0 & 0.0 \\
\hline$P$ & 0.0000050 & 0.0000050 & 0.0000235 & 0.0000465 & 0.0 & 0.0000028 \\
\hline $\mathrm{S}$ & 0.0000010 & 0.0000010 & 0.0000314 & 0.0000335 & 0.0 & 0.0000007 \\
\hline $\mathrm{Cl}$ & 0.0000001 & 0.0000001 & 0.0 & 0.0 & 0.0 & 0.0 \\
\hline $\mathrm{Ca}$ & 0.0000009 & 0.0000009 & 0.0 & 0.0 & 0.0 & 0.0 \\
\hline Co & 0.0000015 & 0.0000015 & 0.0000010 & 0.0 & 0.0 & 0.0 \\
\hline
\end{tabular}


TABLE 8.1 (contd)

\begin{tabular}{|c|c|c|c|c|c|c|}
\hline Isotope & $\begin{array}{c}\text { Inner Ring } \\
\text { Control } \\
\text { Position } \\
0-20 \\
\end{array}$ & $\begin{array}{c}\text { Inner Ring } \\
\text { Control } \\
\text { Position } \\
20-36 \\
\end{array}$ & $\begin{array}{c}\text { Middle Ring } \\
\text { Control } \\
\text { Position } \\
0-20 \\
\end{array}$ & $\begin{array}{c}\text { Middle Ring } \\
\text { Control } \\
\text { Position } \\
20-36 \\
\end{array}$ & $\begin{array}{c}\text { Outer Ring } \\
\text { Control } \\
\text { Position } \\
0-20 \\
\end{array}$ & $\begin{array}{c}\text { Outer Ring } \\
\text { Control } \\
\text { Position } \\
20-36 \\
\end{array}$ \\
\hline $\mathrm{C}$ & 0.0000312 & 0.0000309 & 0.0000312 & 0.0000309 & 0.0000311 & 0.0000309 \\
\hline 0 & 0.0000013 & 0.0000013 & 0.0000013 & 0.0000013 & 0.0000013 & 0.0000013 \\
\hline $\mathrm{Na}$ & 0.0182904 & 0.0184405 & 0.0182904 & 0.0184405 & 0.0182904 & 0.0184405 \\
\hline $\mathrm{Si}$ & 0.0001661 & 0.0001649 & 0.0001661 & 0.0001649 & 0.0001657 & 0.0001649 \\
\hline Al & 0.0000047 & 0.0000049 & 0.0000047 & 0.0000049 & 0.0000047 & 0.0000049 \\
\hline $\mathrm{Mn}$ & 0.0002451 & 0.0002443 & 0.0002451 & 0.0002443 & 0.0002448 & 0.0002443 \\
\hline $\mathrm{Cr}$ & 0.0029778 & 0.0029657 & 0.0029778 & 0.0029657 & 0.0029723 & 0.0029657 \\
\hline $\mathrm{Fe}$ & 0.0105129 & 0.0104697 & 0.0105129 & 0.0104697 & 0.0104937 & 0.0104697 \\
\hline $\mathrm{Ni}$ & 0.0013332 & 0.0013281 & 0.0013332 & 0.0013281 & 0.0013307 & 0.0013281 \\
\hline $\mathrm{Cu}$ & 0.0000357 & 0.0000353 & 0.0000357 & 0.0000353 & 0.0000357 & 0.0000353 \\
\hline Mo & 0.0000176 & 0.0000174 & 0.0000176 & 0.0000174 & 0.0000175 & 0.0000174 \\
\hline U4 & 0.0 & 0.0 & 0.0 & 0.0 & 0.0 & 0.0 \\
\hline U5 & 0.0 & 0.0 & 0.0 & 0.0 & 0.0 & 0.0 \\
\hline U6. & 0.0 & 0.0 & 0.0 & 0.0 & 0.0 & 0.0 \\
\hline U8 & 0.0 & 0.0 & 0.0 & 0.0 & 0.0 & 0.0 \\
\hline $\mathrm{PB}$ & 0.0 & 0.0 & 0.0 & 0.0 & 0.0 & 0.0 \\
\hline P9 & 0.0 & 0.0 & 0.0 & 0.0 & 0.0 & 0.0 \\
\hline PO & 0.0 & 0.0 & 0.0 & 0.0 & $0.0^{\circ}$ & 0.0 \\
\hline P1 & 0.0 & 0.0 & 0.0 & 0.0 & 0.0 & 0.0 \\
\hline $\mathrm{P2}$ & 0.0 & 0.0 & 0.0 & 0.0 & 0.0 & 0.0 \\
\hline $\mathrm{A} 1$ & 0.0 & 0.0 & 0.0 & 0.0 & 0.0 & 0.0 \\
\hline $\mathrm{P}$ & 0.0000040 & 0.0000040 & 0.0000040 & 0.0000040 & 0.0000040 & 0.0000040 \\
\hline s & 0.0000012 & 0.0000012 & 0.0000012 & 0.0000012 & 0.0000012 & 0.0000012 \\
\hline $\mathrm{C} 1$ & 0.0000006 & 0.0000006 & 0.0000006 & 0.0000006 & 0.0000006 & 0.0000006 \\
\hline $\mathrm{Ca}$ & 0.0000042 & 0.0000042 & 0.0000042 & 0.0000042 & 0.0000042 & 0.0000042 \\
\hline Co & 0.0000038 & 0.0000037 & 0.0000038 & 0.0000037 & 0.0000038 & 0.0000037 \\
\hline
\end{tabular}


TABLE 8.1 (contd)

\begin{tabular}{|c|c|c|c|}
\hline Isotope & $\begin{array}{c}\text { Radial } \\
\text { Blanket } \\
\text { Master } 501 \\
0-31 \\
\end{array}$ & $\begin{array}{c}\text { Radial } \\
\text { Blanket } \\
\text { Master } 502 \\
0-31 \\
\end{array}$ & $\begin{array}{c}\text { Radial } \\
\text { Blanket } \\
\text { Master } 503 \\
0-31 \\
\end{array}$ \\
\hline C & 0.0000294 & 0.0000325 & 0.0000324 \\
\hline 0 & 0.0 & 0.0226351 & 0.0220579 \\
\hline $\mathrm{Na}$ & 0.0 & 0.0042352 & 0.0042350 \\
\hline Si & 0.0001099 & 0.0001398 & 0.0001417 \\
\hline Al & 0.0 & 0.0000024 & 0.0000024 \\
\hline Mn & 0.0001648 & 0.0002005 & 0.0001984 \\
\hline $\mathrm{Cr}$ & 0.0018487 & 0.0023279 & 0.0023140 \\
\hline $\mathrm{Fe}$ & 0.0066628 & 0.0083268 & 0.0082484 \\
\hline $\mathrm{Ni}$ & 0.0007495 & 0.0009950 & 0.0010098 \\
\hline $\mathrm{Cu}$ & 0.0000249 & 0.0000275 & 0.0000317 \\
\hline Mo & 0.0000124 & 0.0000127 & 0.0000150 \\
\hline U4 & $\quad 0.0$ & 0.0 & 0.0 \\
\hline U5 & 0.0000865 & 0.0000290 & 0.0000285 \\
\hline U6 & 0.0 & 0.0 & 0.0 \\
\hline U8 & 0.0387435 & 0.0133322 & 0.0131212 \\
\hline P8 & $\quad 0.0$ & 0.0 & 0.0 \\
\hline P9 & 0.0 & 0.0 & 0.0 \\
\hline $\mathrm{PO}$ & 0.0 & 0.0 & 0.0 \\
\hline P1 & 0.0 & 0.0 & 0.0 \\
\hline P2 & 0.0 & 0.0 & 0.0 \\
\hline Al & 0.0 & 0.0 & 0.0 \\
\hline$P$ & 0.0000046 & 0.0000052 & 0.0000047 \\
\hline$S$ & 0.0000010 & 0.0000010 & 0.0000012 \\
\hline $\mathrm{Cl}$ & 0.0 & 0.0000001 & 0.0000001 \\
\hline $\mathrm{Ca}$ & 0.0 & 0.0000010 & 0.0000010 \\
\hline $\mathrm{Co}$ & 0.0 & 0.0000007 & 0.0000045 \\
\hline
\end{tabular}




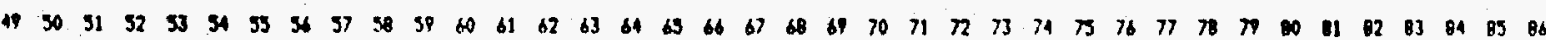

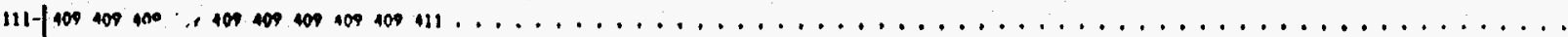

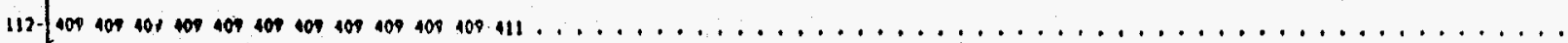

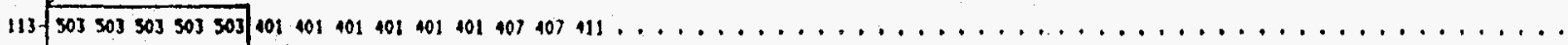

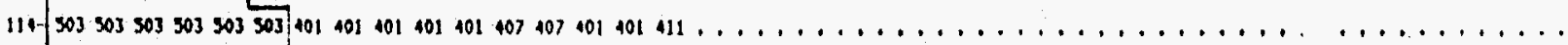

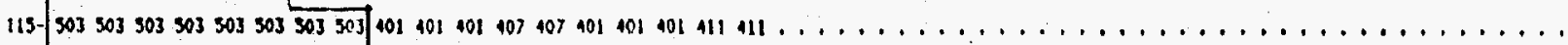

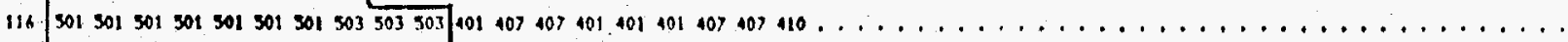

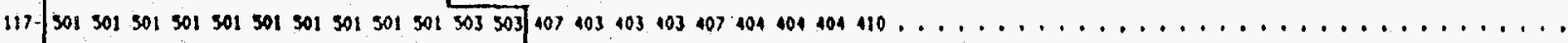

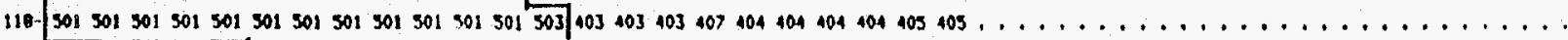

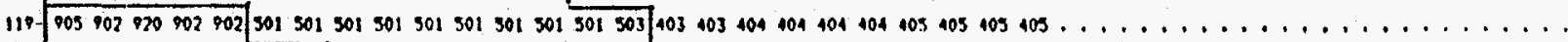

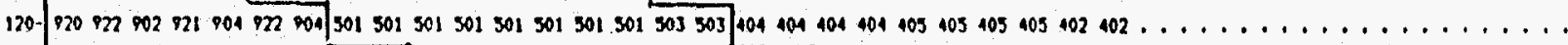

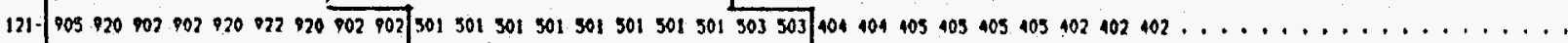

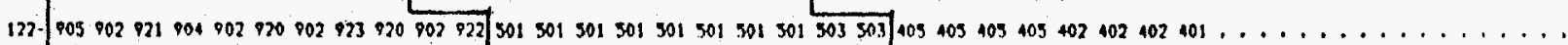

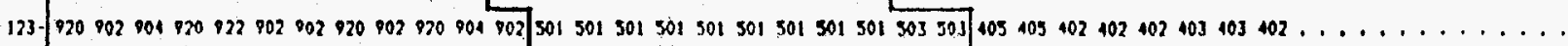

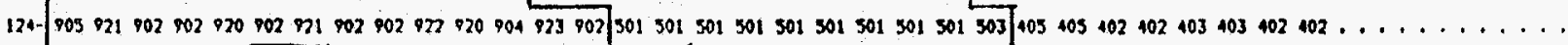

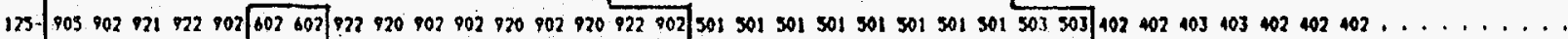

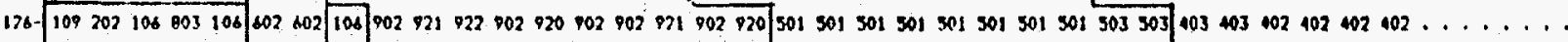

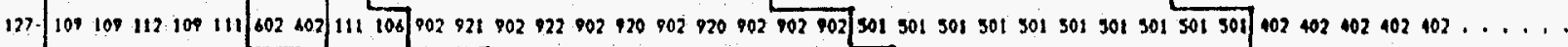

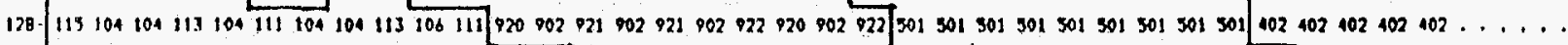

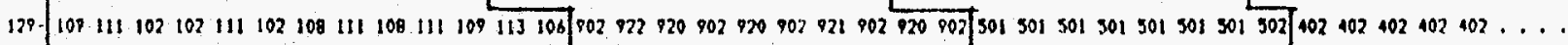

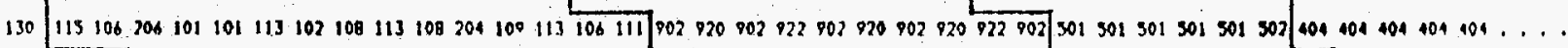

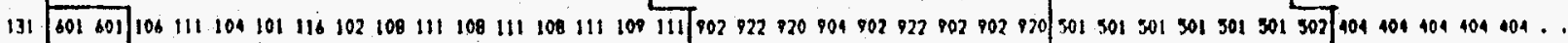

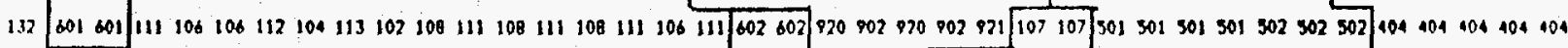

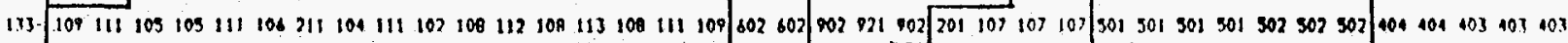

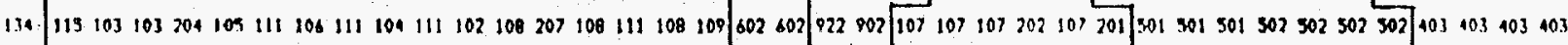

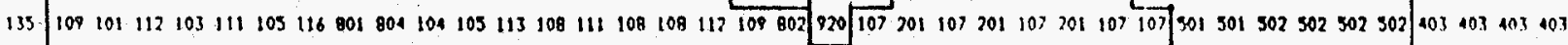

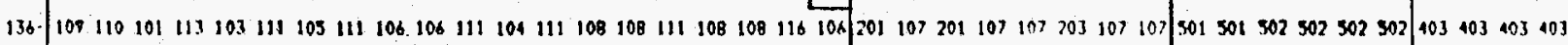

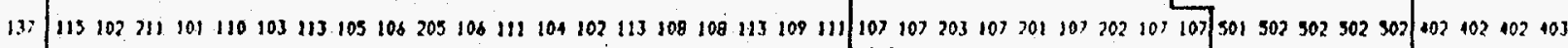

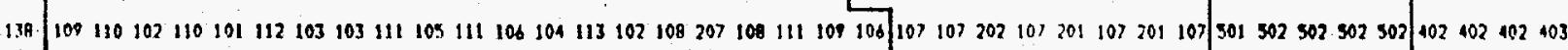

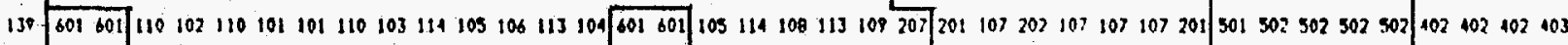

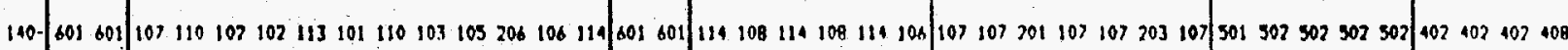

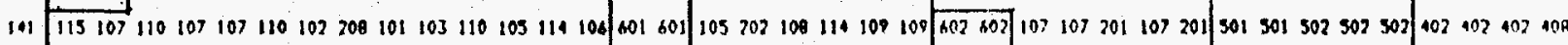

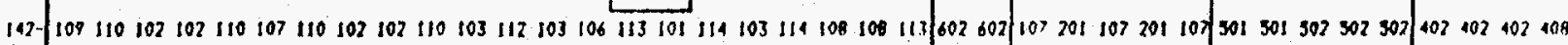

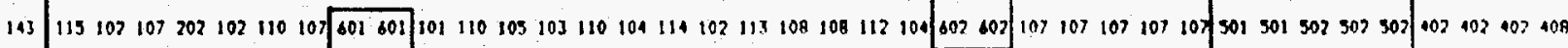

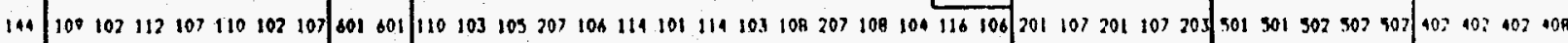

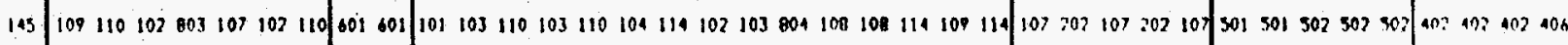

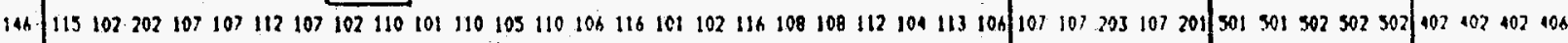

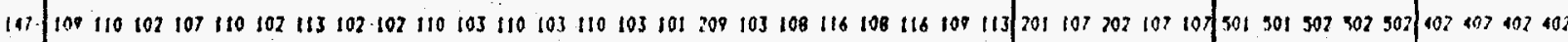

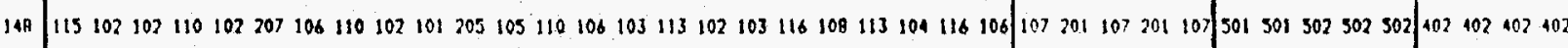

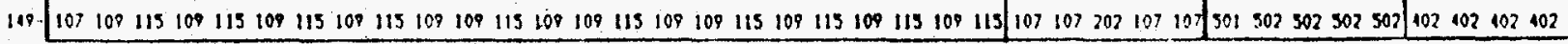

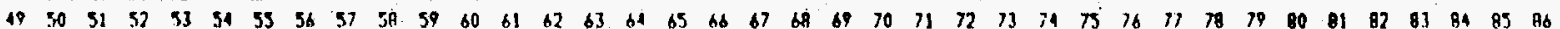

Fig. 8.1 The xyz Calculation Model for ZPPR-19A 
9. CALCULATION MODELS FOR ZPPR-19B (G. L. Grasseschi and P. J. Collins)

ZPPR-19B was built to provide further data on the effects of the uranium fuel in the outer core. The uranium and plutonium fuel drawers were rearranged to give an approximately uniform distribution throughout the outer core. Measurements of reaction rates and control rod worths in $\mathrm{ZPPR}-19 \mathrm{~B}$ were made to compare with those in $Z P P R-18 \mathrm{~A}$ which had uranium and plutonium sectors in the outer core. ZPPR-19B had substantially more uranium fuel than did ZPPR-18A since fuel was added in ZPPR-18B to improve the power distributions. Measurements in 18A and 19B cannot be compared directly, but it is believed that the change in the amount of fuel will have little effect on the analytical comparisons. A detailed description of ZPPR-19B is given in ANL-ZPR-489, p. 8 .

Calculations for ZPPR-19B used the ZPPR-18 cross section library (ANL-ZPR-489, p. 12) in which cross sections processing was made using group dependent buckling for cells in the sector model. Calculations were made in xyz geometry with detailed compositions by drawer master as was done for ZPPR-18. The $x y$ plan is given in Figure 9.1. The drawer masters shown in the figure are described in ANLFPR-489 Tables 4.1 and 5.1. Average composition for each zone in $Z P P R-19 B$ are given in Table 9.1 . 
TABLE 9.1 Atom Densities by Zone and Drawer Type in ZPPR-19B

\begin{tabular}{|c|c|c|c|c|c|c|}
\hline Isotope & $\begin{array}{l}\text { Inner } \\
\text { Core } \\
\text { Average } \\
0-20\end{array}$ & $\begin{array}{c}\text { Axial } \\
\text { Blanket } \\
\text { (IC) } \\
20-31\end{array}$ & $\begin{array}{c}\text { Reflector } \\
\text { Iron Block } \\
\text { (IC) } \\
31-36\end{array}$ & $\begin{array}{c}\text { Inner } \\
\text { Core } \\
\text { (ICSF) } \\
0-20\end{array}$ & $\begin{array}{l}\text { Inner } \\
\text { Core } \\
\text { (ICSM) } \\
0-20\end{array}$ & $\begin{array}{l}\text { Inner } \\
\text { Core } \\
\text { Single Pu } \\
0-20\end{array}$ \\
\hline C & 0.0000332 & 0.0000532 & 0.0005873 & 0.0000332 & 0.0000332 & 0.0000332 \\
\hline 0 & 0.0117067 & 0.0088210 & $\quad 0.0$ & 0.0137453 & 0.0088141 & 0.0117067 \\
\hline $\mathrm{Na}$ & 0.0092276 & 0.0091887 & 0.0 & 0.0092715 & 0.0091653 & 0.0092276 \\
\hline Si & 0.0001577 & 0.0001923 & 0.0001258 & 0.0001570 & 0.0001586 & 0.0001577 \\
\hline $\mathrm{Al}$ & 0.0000040 & 0.0000029 & 0.0 & 0.0000040 & 0.0000040 & 0.0000040 \\
\hline $\mathrm{Mn}$ & 0.0002288 & 0.0003421 & 0.0006824 & 0.0002287 & 0.0002289 & 0.0002288 \\
\hline $\mathrm{Cr}$ & 0.0026718 & 0.0041655 & 0.0021133 & 0.0026684 & 0.0026767 & 0.0026718 \\
\hline $\mathrm{Fe}$ & 0.0114739 & 0.0148016 & 0.0756998 & 0.0128536 & 0.0095163 & 0.0114739 \\
\hline $\mathrm{Ni}$ & 0.0011730 & 0.0017641 & 0.0008575 & 0.0011678 & 0.0011803 & 0.0011730 \\
\hline $\mathrm{Cu}$ & 0.0000300 & 0.0000439 & 0.0000268 & 0.0000293 & 0.0000311 & 0.0000300 \\
\hline Mo & 0.0002391 & 0.0000345 & 0.0000134 & 0.0002397 & 0.0002383 & 0.0002391 \\
\hline U4 & $\quad 0.0$ & 0.0 & 0.0 & 0.0 & 0.0 & 0.0 \\
\hline U5 & 0.0000171 & 0.0000179 & 0.0 & 0.0000127 & 0.0000234 & 0.0000171 \\
\hline U6 & 0.0 & 0.0 & 0.0 & 0.0 & 0.0 & 0.0 \\
\hline U8 & 0.0078140 & 0.0081561 & 0.0 & 0.0058158 & 0.0106494 & 0.0078140 \\
\hline P8 & 0.0000004 & 0.0 & 0.0 & 0.0000004 & 0.0000005 & 0.0000004 \\
\hline P9 & 0.0008843 & 0.0 & 0.0 & 0.0008878 & 0.0008794 & 0.0008843 \\
\hline PO & 0.0001170 & 0.0 & 0.0 & 0.0001176 & 0.0001162 & 0.0001170 \\
\hline P1 & 0.0000067 & 0.0 & 0.0 & 0.0000065 & 0.0000069 & 0.0000067 \\
\hline P2 & 0.0000018 & 0.0 & 0.0 & 0.0000017 & 0.0000019 & 0.0000018 \\
\hline $\mathrm{A} 1$ & 0.0000108 & 0.0 & 0.0 & 0.0000108 & 0.0000107 & 0.0000108 \\
\hline$P$ & 0.0000052 & 0.0000102 & 0.0000237 & 0.0000053 & 0.0000051 & 0.0000052 \\
\hline$S$ & 0.0000010 & 0.0000081 & 0.0000312 & 0.0000010 & 0.0000011 & 0.0000010 \\
\hline $\mathrm{Cl}$ & 0.0000003 & 0.0000003 & 0.0 & 0.0000003 & 0.0000003 & 0.0000003 \\
\hline $\mathrm{Ca}$ & 0.0000021 & 0.0000021 & 0.0 & 0.0000021 & 0.0000021 & 0.0000021 \\
\hline $\mathrm{Co}$ & 0.0000014 & 0.0000014 & 0.0000007 & 0.0000008 & 0.0000023 & 0.0000014 \\
\hline
\end{tabular}


TABLE 9.1 (contd)

\begin{tabular}{|c|c|c|c|c|c|}
\hline Isotope & $\begin{array}{c}\text { Outer } \\
\text { Core } \\
\text { Average } \\
0-20 \\
\end{array}$ & $\begin{array}{c}\text { Outer } \\
\text { Core } \\
\text { Average } \\
20-20.44 \\
\end{array}$ & $\begin{array}{c}\text { Axial } \\
\text { Blanket } \\
\text { (OC) } \\
20.44-31 \\
\end{array}$ & $\begin{array}{l}\text { Rerlector } \\
\text { Iron Block } \\
\text { (OC) } \\
31-36 \\
\end{array}$ & $\begin{array}{c}\text { Outer } \\
\text { Core } \\
\text { Pu Fuel } \\
0-20 \\
\end{array}$ \\
\hline C & 0.0015216 & 0.0000430 & 0.0000547 & 0.0005929 & 0.0006277 \\
\hline 0 & 0.0157793 & 0.0088366 & 0.0088298 & 0.0 & 0.0142449 \\
\hline $\mathrm{Na}$ & 0.0087748 & 0.0093153 & 0.0092655 & 0.0 & 0.0091654 \\
\hline Si & 0.0001667 & 0.0001699 & 0.0002019 & 0.0001167 & 0.0001635 \\
\hline A. & 0.0000043 & 0.0000029 & 0.0000029 & 0.0 & 0.0000046 \\
\hline$M n$ & 0.0002382 & 0.0002670 & 0.0003450 & 0.0006745 & 0.0002379 \\
\hline $\mathrm{Cr}$ & 0.0028267 & 0.0031948 & 0.0041058 & 0.0019593 & 0.0027889 \\
\hline $\mathrm{Fe}$ & 0.0146052 & 0.0113725 & 0.0146096 & 0.0761394 & 0.0123834 \\
\hline $\mathrm{Ni}$ & 0.0012475 & 0.0013673 & 0.0017579 & 0.0007929 & 0.0012336 \\
\hline $\mathrm{Cu}$ & 0.0000315 & 0.0000360 & 0.0000410 & 0.0000276 & 0.0000312 \\
\hline Mo & 0.0001351 & 0.0000232 & 0.0000297 & 0.0000139 & 0.0002990 \\
\hline U4 & 0.0000088 & 0.0000073 & 0.0 & 0.0 & 0.0 \\
\hline U5 & 0.0009128 & 0.0007587 & 0.0000179 & 0.0 & 0.0000142 \\
\hline U6 & 0.0000042 & 0.0000035 & $\quad 0.0$ & 0.0 & 0.0 \\
\hline U8 & 0.0068164 & 0.0082076 & 0.0081643 & 0.0 & 0.0064867 \\
\hline P8 & 0.0000003 & 0.0 & 0.0 & 0.0 & 0.0000006 \\
\hline P9 & 0.0004706 & 0.0 & 0.0 & 0.0 & 0.0011175 \\
\hline PO & 0.0000623 & 0.0 & 0.0 & 0.0 & 0.0001479 \\
\hline P1 & 0.0000038 & 0.0 & 0.0 & 0.0 & 0.0000089 \\
\hline P2 & 0.0000010 & 0.0 & 0.0 & 0.0 & 0.0000024 \\
\hline A) & 0.0000058 & 0.0 & 0.0 & 0.0 & 0.0000138 \\
\hline $\mathrm{P}$ & 0.0000052 & 0.0000074 & 0.0000102 & 0.0000234 & 0.0000051 \\
\hline$S$ & 0.0000014 & 0.0000039 & 0.0000065 & 0.0000314 & 0.0000012 \\
\hline $\mathrm{Cl}$ & 0.0000041 & 0.0000003 & 0.0000003 & 0.0 & 0.0000018 \\
\hline $\mathrm{Ca}$ & 0.0000013 & 0.0000021 & 0.0000021 & 0.0 & 0.0000018 \\
\hline Co & 0.0000022 & 0.0000022 & 0.0000021 & 0.0000015 & 0.0000018 \\
\hline
\end{tabular}


TABLE 9.1 (contd)

\begin{tabular}{|c|c|c|c|c|c|c|}
\hline Isotope & $\begin{array}{c}\text { Axial } \\
\text { Blanket } \\
\text { (OC) } \\
\text { Pu Euel } \\
20-31\end{array}$ & $\begin{array}{l}\text { Reflector } \\
\text { Iron Block } \\
\text { (OC) } \\
\text { Pu Fuel } \\
31-36 \\
\end{array}$ & $\begin{array}{c}\text { Outer } \\
\text { Core } \\
\text { (OC) } \\
\text { Pu Fuel } \\
\text { o-20 }\end{array}$ & $\begin{array}{r}\text { Outer } \\
\text { Core } \\
\text { Single } \mathrm{Pu} \\
0-20 \\
\end{array}$ & $\begin{array}{c}\text { Outer } \\
\text { Core } \\
\text { U Fuel } \\
0-20\end{array}$ & $\begin{array}{c}\text { Outer } \\
\text { Core } \\
\text { U Fue } 1 \\
20-20.44 \\
\end{array}$ \\
\hline $\mathrm{C}$ & 0.0000531 & 0.0005928 & 0.0023103 & 0.0000332 & 0.0021684 & 0.0000343 \\
\hline 0 & 0.0088211 & 0.0 & 0.0156589 & 0.0137451 & 0.0168662 & 0.0088277 \\
\hline $\mathrm{Na}$ & 0.0092557 & 0.0 & 087864 & 0.0092992 & 0.0084761 & 0.0093001 \\
\hline Si & 0.0001926 & 0.0001164 & 0.0001819 & 0.0001570 & 0.0001688 & 0.0001532 \\
\hline AI & 0.0000029 & 0.0 & 0.0000060 & 0.0000041 & 0.0000041 & 0.0000029 \\
\hline$M n$ & 0.0003419 & 0.0006746 & 0.0002649 & 0.0002283 & 0.0002381 & 0.0002119 \\
\hline $\mathrm{Cr}$ & 0.0041646 & 0.0019583 & 0.0031388 & 0.0026652 & 0.0028492 & 0.0024835 \\
\hline $\mathrm{Fe}$ & 0.0147953 & 0.0761507 & 0.0110838 & 0.0128424 & 0.0161911 & 0.0088611 \\
\hline $\mathrm{Ni}$ & 0.0017661 & 0.0007907 & 0.0014237 & 0.0011664 & 0.0012555 & 0.0010753 \\
\hline $\mathrm{Cu}$ & 0.0000443 & 0.0000273 & 0.0000364 & 0.0000293 & 0.0000317 & 0.0000298 \\
\hline Mo & 0.0000347 & 0.0000137 & 0.0004688 & 0.0002390 & 0.0000158 & 0.0000147 \\
\hline U4 & 0.0 & 0.0 & 0.0 & 0.0 & 0.0000152 & 0.0000125 \\
\hline U5 & 0.0000179 & 0.0 & 0.0000183 & 0.0000127 & 0.0015647 & 0.0012962 \\
\hline U6 & 0.0 & 0.0 & 0.0 & 0.0 & 0.0000073 & 0.0000060 \\
\hline U8 & 0.0081562 & 0.0 & 0.0083531 & 0.0058272 & 0.0070436 & 0.0082305 \\
\hline P8 & $\quad 0.0$ & 0.0 & 0.0000010 & 0.0000005 & 0.0 & 0.0 \\
\hline P9 & 0.0 & 0.0 & 0.0017747 & 0.0008852 & 0.0 & 0.0 \\
\hline PO & 0.0 & 0.0 & 0.0002348 & 0.0001171 & 0.0 & 0.0 \\
\hline P1 & 0.0 & 0.0 & 0.0000141 & 0.0000071 & 0.0 & 0.0 \\
\hline $\mathrm{P} 2$ & 0.0 & 0.0 & 0.0000039 & 0.0000019 & 0.0 & 0.0 \\
\hline A 1 & 0.0 & 0.0 & 0.0000218 & 0.0000110 & $\quad 0.0$ & 0.0 \\
\hline$P$ & 0.0000102 & 0.0000234 & 0.0000047 & 0.0000053 & 0.0000053 & 0.0000053 \\
\hline$S$ & 0.0000081 & 0.0000314 & 0.0000016 & 0.0000010 & 0.0000016 & 0.0000013 \\
\hline $\mathrm{Cl}$ & 0.0000003 & 0.0 & 0.0000062 & 0.0000003 & 0.0000058 & 0.0000003 \\
\hline $\mathrm{Ca}$ & 0.0000021 & 0.0 & 0.0000010 & 0.0000021 & 0.0000010 & 0.0000021 \\
\hline Co & 0.0000018 & 0.0000011 & 0.0000045 & 0.0000008 & 0.0000026 & 0.0000026 \\
\hline
\end{tabular}


TABLE 9.1 (contd)

\begin{tabular}{|c|c|c|c|c|c|c|}
\hline Isotope & $\begin{array}{c}\text { Axial } \\
\text { Blanket } \\
\text { (OC) } \\
\text { Pu Fuel } \\
20.44-31 \\
\end{array}$ & $\begin{array}{l}\text { Reflector } \\
\text { Iron Block } \\
\text { (OC) } \\
\text { U Fuel } \\
31-36 \\
\end{array}$ & $\begin{array}{c}\text { Outer } \\
\text { Core } \\
\text { Single U } \\
\text { (OCUS) } \\
0-20 \\
\end{array}$ & $\begin{array}{c}\text { Outer } \\
\text { Core } \\
\text { Single U } \\
\text { (OCUS) } \\
20-20.44 \\
\end{array}$ & $\begin{array}{c}\text { Outer } \\
\text { Core } \\
\text { Double U } \\
\text { (OCUD) } \\
0-20 \\
\end{array}$ & $\begin{array}{c}\text { Outer } \\
\text { Core } \\
\text { Double U } \\
\text { (OCUD) } \\
20-20.44 \\
\end{array}$ \\
\hline C & 0.0000557 & 0.0005919 & 0.0021064 & 0.0000340 & 0.0022637 & 0.0000349 \\
\hline 0 & 0.0088209 & $\quad 0.0$ & 0.0171001 & 0.0088278 & 0.0165071 & 0.0088277 \\
\hline $\mathrm{Na}$ & 0.0092567 & 0.0 & 0.0083664 & 0.0092904 & 0.0086446 & 0.0093153 \\
\hline $\mathrm{Si}$ & 0.0002084 & 0.0001167 & 0.0001684 & 0.0001481 & 0.0001694 & 0.0001610 \\
\hline $\mathrm{Al}$ & 0.0000029 & 0.0 & 0.0000043 & 0.0000029 & 0.0000038 & 0.0000028 \\
\hline $\mathrm{Mn}$ & 0.0003466 & 0.0006733 & 0.0002412 & 0.0002105 & 0.0002333 & 0.0002142 \\
\hline $\mathrm{Cr}$ & 0.0040559 & 0.0019566 & 0.0028882 & 0.0024577 & 0.0027894 & 0.0025231 \\
\hline $\mathrm{Fe}$ & 0.0144494 & 0.0759994 & 0.0177817 & 0.0087816 & 0.0137475 & 0.0089833 \\
\hline $\mathrm{Ni}$ & 0.0017489 & 0.0007932 & 0.0012690 & 0.0010564 & 0.0012347 & 0.0011044 \\
\hline $\mathrm{Cu}$ & 0.0000385 & 0.0000279 & 0.0000302 & 0.0000281 & 0.0000340 & 0.0000325 \\
\hline Mo & 0.0000260 & 0.0000140 & 0.0000147 & 0.0000133 & 0.0000175 & 0.0000167 \\
\hline U4 & 0.0 & 0.0 & 0.0000109 & 0.0000082 & 0.0000217 & 0.0000192 \\
\hline U5 & 0.0000179 & 0.0 & 0.0011314 & 0.0008546 & 0.0022303 & 0.0019746 \\
\hline U6 & $\quad 0.0$ & 0.0 & 0.0000052 & 0.0000039 & 0.0000104 & 0.0000092 \\
\hline u8 & 0.0081560 & 0.0 & 0.0073446 & 0.0082050 & 0.0065814 & 0.0082698 \\
\hline P8 & 0.0 & 0.0 & 0.0 & 0.0 & 0.0 & 0.0 \\
\hline P9 & 0.0 & 0.0 & 0.0 & 0.0 & 0.0 & 0.0 \\
\hline PO & 0.0 & 0.0 & 0.0 & 0.0 & 0.0 & 0.0 \\
\hline P1 & 0.0 & 0.0 & 0.0 & 0.0 & 0.0 & 0.0 \\
\hline P2 & 0.0 & 0.0 & 0.0 & 0.0 & 0.0 & 0.0 \\
\hline A1 & 0.0 & 0.0 & 0.0 & 0.0 & 0.0 & 0.0 \\
\hline$P$ & 0.0000102 & 0.0000233 & 0.0000054 & 0.0000054 & 0.0000051 & 0.0000052 \\
\hline $\mathrm{S}$ & 0.0000054 & 0.0000314 & 0.0000015 & 0.0000011 & 0.0000019 & 0.0000015 \\
\hline $\mathrm{Cl}$ & 0.0000003 & 0.0 & 0.0000056 & 0.0000003 & 0.0000061 & 0.0000003 \\
\hline $\mathrm{Ca}$ & 0.0000021 & 0.0 & 0.0000010 & 0.0000021 & 0.0000010 & 0.0000021 \\
\hline Co & 0.0000023 & 0.0000017 & 0.0000010 & 0.0000010 & 0.0000050 & 0.0000050 \\
\hline
\end{tabular}




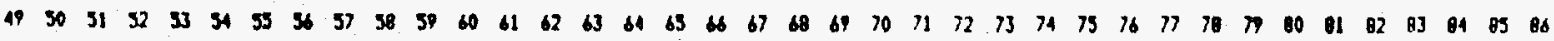

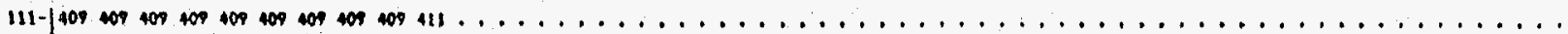

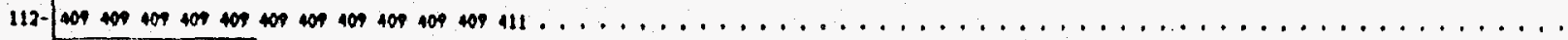

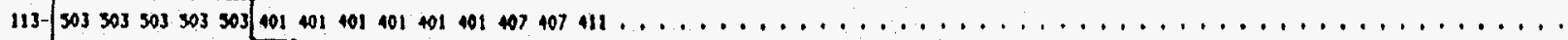

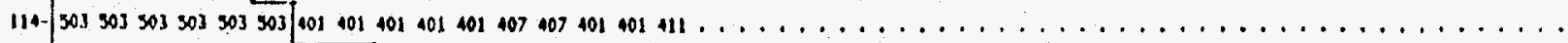

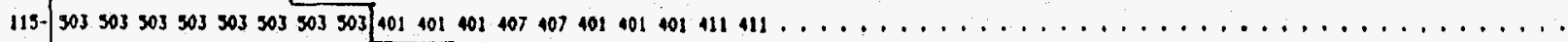

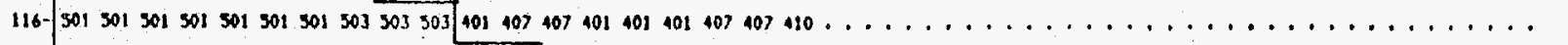

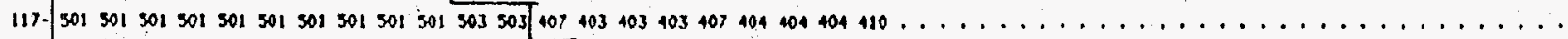

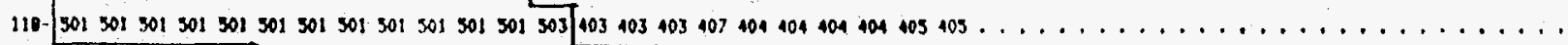

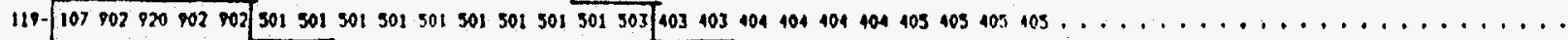

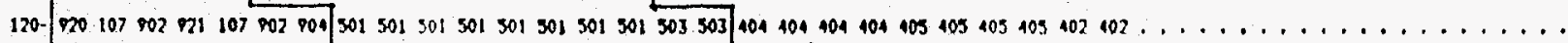

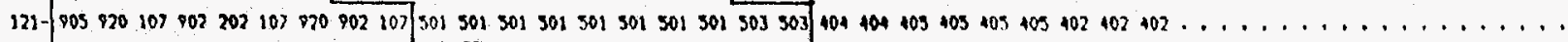

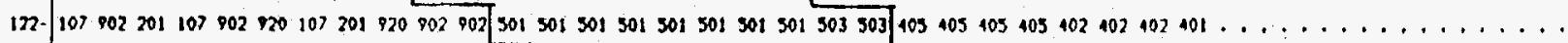

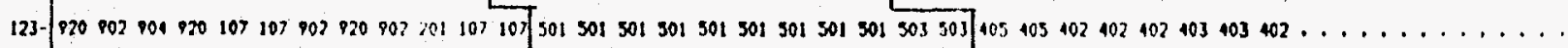

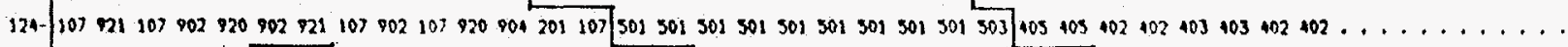

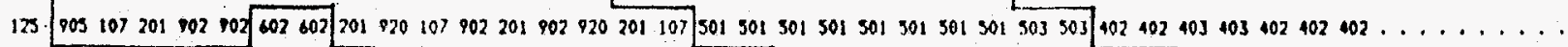

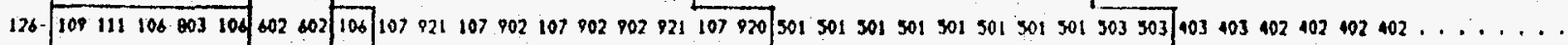

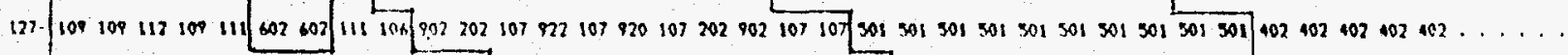

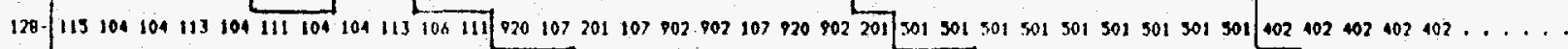

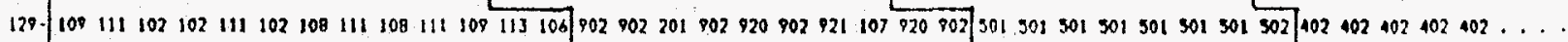

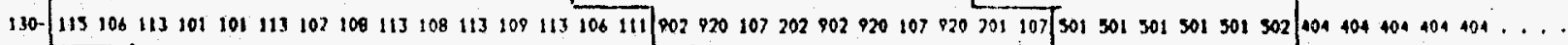

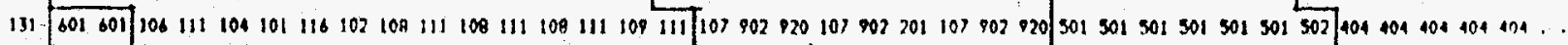

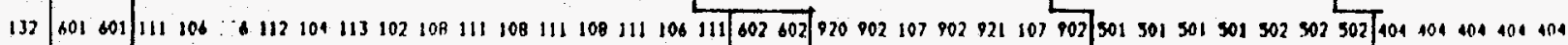

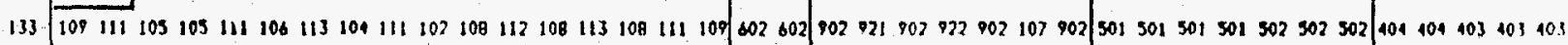

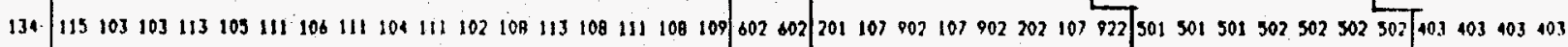

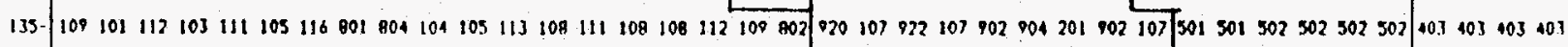

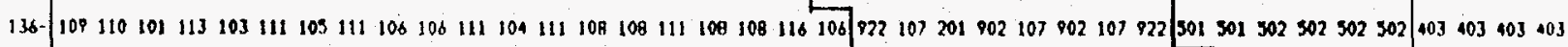

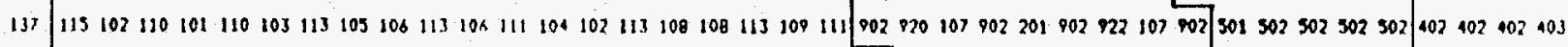

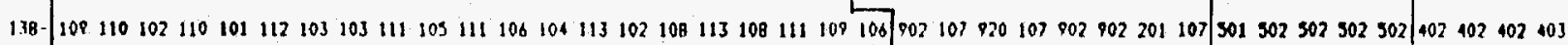

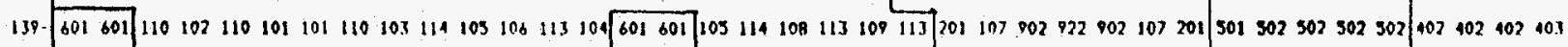

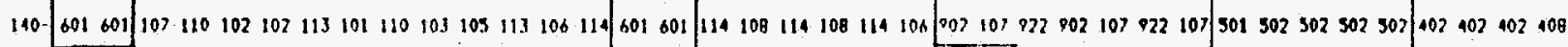

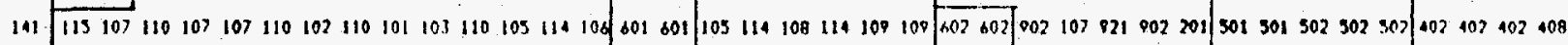

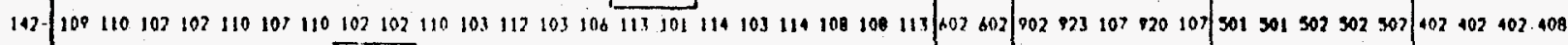

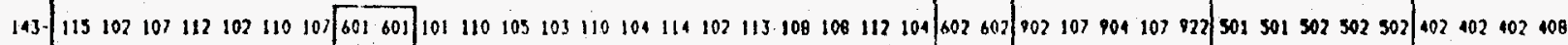

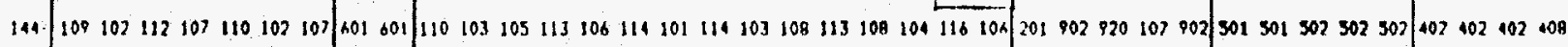

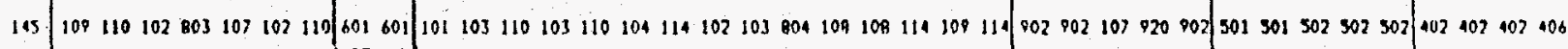

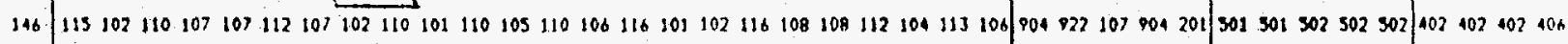

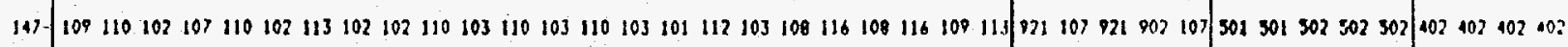

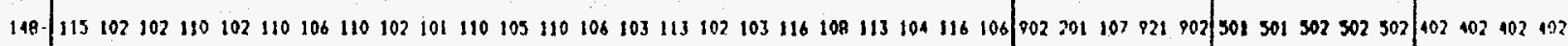

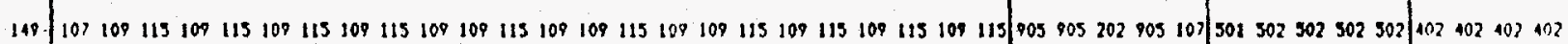

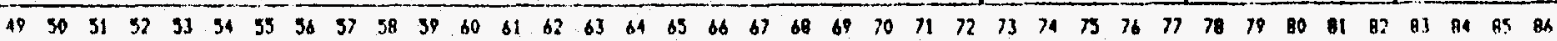




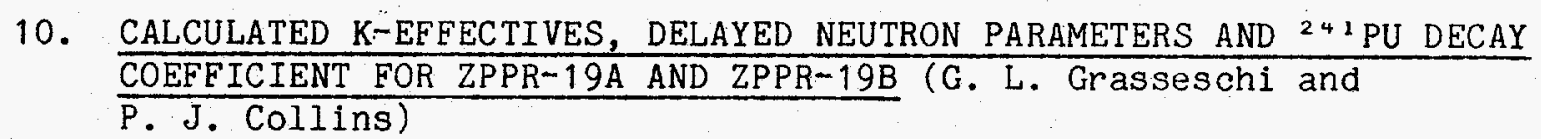

\section{1 k-effectives}

Calculations for ZPPR-19A and ZPPR-19B were made with xyz models using finite-difference diffusion (FDDT), nodal diffusion (NDT) and nodal transport (NTT). The calculation models are given in Sections 8 and 9 of this report. Mesh sizes, node spacings and treatment of mismatch in uranium and plutonium fuel column lengths were consistent with those used for ZPPR 18 .

Six group data were used for calculations of control rods in ZPPR-19B. Data were collapsed for each generic cell type using fluxes from a 21 group xyz diffusion calculation. Pseudo-absorption (buckling) terms for use in $x y$ calculations were calculated from core/axial blanket leakages from a 6 group xyz diffusion calculation.

The calculated k-effectives (not C/E results) for the different models of ZPPR-19 are given in Table 10.1. A number of different calculations were made for $Z P P R-19 B$ because of requirements of calculating control rod worths (subcritical reference with shim rods withdrawn), reaction rates (critical reference with shim rods inserted) and for performing group collapse and buckling generation (generic master compositions). These provide a collection of the effects of different modeling approximations which are given in Table 10.2.

The errors due to modeling approximations are quite similar to those found for $Z P P R-18$ (ANL-ZPR-489, p. 45). Transport and diffusion mesh effects are larger in $Z P P R-19 A$ than in $19 B$ due to the inserted control rods. The error due to six group condensation (0.0012) is less in $19 \mathrm{~B}$ than in $18 \mathrm{~A}(0.0021)$. The results in Table 10.2 also show the separability of corrections. For example, transport and mesh-size corrections are the same to $0.0001 \Delta \mathrm{k}$ whether calculated with 6 groups or with 21 groups. 
Experimental excess reactivities and operational data for ZPPR-19 are given in Tables 10.3 and 10.4. To derive an experimental value for kieffective, the data have been adjusted to a temperature of $293 \mathrm{~K}$ (used in generating the cross section libraries). For comparison, the subcritical references have been adjusted to the interface gap and ${ }^{241} \mathrm{Pu}$ decay of the critical references. The calculated $\beta$-effective values are used to convert measured values in cents to $\Delta \mathrm{k}$ units. Note that the uncertainties shown are due to the operational measurements alone. The total uncertainty is typically $0.05 \% \Delta k$ but strongly correlated between the ZPPR 18 and ZPPR 19 cores since the majority of the fuel is common to the assemblies.

The criticality predictions for ZPPRr 19, shown in Table 10.5, are similar to those for $Z P P R-18$. In particular, the nodal transport value for ZPPR 19B (0.9926) is close to that for $18 \mathrm{~A}(0.9925)$ while cores $19 \mathrm{~A}$ and $18 \mathrm{~B}$, with inserted control rods, have a higher k-effective by about 0.001 .

\subsection{Delayed Neutron Parameters}

Delayed neutron parameters for ZPPRr 19A and 19B were calculated with the xyz finite-difference diffusion models and 21 group data. The parameters are given in Tables 10.6 and 10.7. The increased $\beta$-effective for ZPPR-19B compared with ZPPR-18A $(0.3786)$ is attributed to the additional ${ }^{235} \mathrm{U}$ which was added. The higher $B$-effective in $19 \mathrm{~A}$ compared to that for $19 \mathrm{~B}$ is attributed to the effect of the inner control rod bank which moves fluxes towards the outer core.

\subsection{Plutonium Decay Coefficient for ZPPR-19}

\footnotetext{
The ${ }^{241} \mathrm{Pu}$ decay coefficient for ZPPR 19 was estimated from the worths of ${ }^{241} \mathrm{Pu}$ and ${ }^{241} \mathrm{Am}$ which were calculated for ZPPR-18A (ANL-ZPR-489, p. 43). The worths were adjusted by the interval between the references for $18 \mathrm{~A}$ and $19 \mathrm{~B}$ ( 0.288 years). The decay coefficient for ZPPR-19B was calculated to be $-0.02143 \phi$ day $^{-1}$. This estimate is adequate within the $10 \%$ (10) uncertainty.
} 
TABLE 10.1. Calculated k-effectives for ZPPR-19

\begin{tabular}{|c|c|c|c|c|c|c|c|c|c|c|}
\hline & Core & $\begin{array}{l}\text { Reactor } \\
\text { Loading }\end{array}$ & $\begin{array}{l}\text { Number } \\
\text { of Groups } \\
\end{array}$ & Geometry & Mesh & $\begin{array}{l}\text { Calculation } \\
\text { Method }^{-}\end{array}$ & Streaming & Shim & Rods ${ }^{C}$ & k-effectived \\
\hline 1 & ZPPR $-19 A$ & C & 21 & $x y z$ & $38 \times 39 \times 9$ & NDT & $Y$ & & $N$ & 0.991024 \\
\hline 3 & & C & 21 & xyz & $38 \times 39 \times 20$ & FDDT & $Y$ & & $\mathrm{~N}$ & 0.993295 \\
\hline 4 & & $S$ & 21 & $x y z$ & $38 \times 39 \times 20$ & FDDT & $Y$ & & $\mathrm{~N}$ & 0.992258 \\
\hline 7 & & $\mathrm{C}$ & 21 & $\mathrm{xyz}$ & $38 \times 39 \times 20$ & FDDT & $Y$ & & $\mathrm{~N}$ & 0.992258 \\
\hline 8 & & $S$ & 21 & $x y z$ & $38 \times 39 \times 9$ & NDT & $Y$ & & $\mathrm{~N}$ & 0.990089 \\
\hline 9 & & $S$ & 21 & $x y z$ & $38 \times 39 \times 9$ & NTT & $Y$ & & $\mathrm{~N}$ & 0.992118 \\
\hline 10 & & $\mathrm{~s}$ & 21 & $x y z$ & $38 \times 39 \times 20$ & FDDT & $Y$ & & $\mathrm{~N}$ & 0.990953 \\
\hline 11 & & $\mathrm{C}$ & 21 & $x y z$ & $38 \times 39 \times 9$ & NDT & $Y$ & & $Y$ & 0.990977 \\
\hline 12 & & C & 21 & $x y z$ & $38 \times 39 \times 9$ & NTT & $Y$ & & $Y$ & 0.993028 \\
\hline 13 & & C & 21 & xyz & $38 \times 39 \times 20$ & FDDT & $N$ & & $\mathrm{~N}$ & 0.995732 \\
\hline 18 & & $\mathrm{C}$ & 6 & xyz & $38 \times 39 \times 20$ & FDDT & $Y$ & & N & 0.991028 \\
\hline 19 & & $C$ & 6 & $\mathrm{xy}$ & $38 \times 39$ & FDDT & $Y$ & & $\mathrm{~N}$ & 0.990589 \\
\hline
\end{tabular}

${ }^{a_{C}}=$ critical reference, $S=$ subcritical reference.

$b_{\text {NDT }}=$ nodal diffusion, NTT $=$ nodal transport, $F D D T=$ finite-difference diffusion.

${ }^{c}$ Shim rods if inserted $(Y)$ are close to position during foil irradiations.

$\mathrm{d}_{\text {Not } \mathrm{C} / \mathrm{E} \text { values. }}$

Model with generic drawer master compositions averaged, for group collapse and buckling generation. 
TABLE 10.2 Calculation Modelling Effects for ZPPR-19

\begin{tabular}{|c|c|c|c|}
\hline Correction & Core & Cases $^{a}$ & $\Delta \mathrm{k}$ \\
\hline Transport & $\begin{array}{l}19 \mathrm{~A} \\
19 \mathrm{~B} \\
19 \mathrm{~B} \\
19 \mathrm{~B}\end{array}$ & $\begin{array}{r}1-2 \\
5-6 \\
15-16 \text { (6 groups) } \\
8-9 \text { (suberitical) }\end{array}$ & $\begin{array}{l}+0.002845 \\
+0.002044 \\
+0.001909 \\
+0.002029\end{array}$ \\
\hline FDDT Mesh & $\begin{array}{l}19 A \\
19 B \\
19 B \\
19 B\end{array}$ & $\begin{aligned} & 1-3 \\
& 5-7 \\
& 15-18 \text { (6 groups) } \\
& 8-10 \text { (subcritical) }\end{aligned}$ & $\begin{array}{l}-0.002271 \\
-0.000865 \\
-0.000871 \\
-0.000864\end{array}$ \\
\hline Streaming & $19 B$ & $7-13$ & -0.003474 \\
\hline Energy Groups & $\begin{array}{l}19 \mathrm{~B} \\
19 \mathrm{~B} \\
19 \mathrm{~B}\end{array}$ & $\begin{array}{ll}5-15 & (\text { NDT }) \\
6-16 & (\text { NTT }) \\
7-18 & (\text { FDDT })\end{array}$ & $\begin{array}{l}-0.001236 \\
+0.001371 \\
+0.001230\end{array}$ \\
\hline Bucklings & $19 \mathrm{~B}$ & 18-19 (6 groups) & +0.000439 \\
\hline All Master Models & $\begin{array}{l}19 \mathrm{~B} \\
19 \mathrm{~B}\end{array}$ & $\begin{array}{r}7-14 \\
17-18 \text { (6 groups) }\end{array}$ & $\begin{array}{l}+0.000304 \\
+0.000287\end{array}$ \\
\hline
\end{tabular}

$a_{\text {Refer to Table } 10.1 .}$ 
TABLE 10.3. Experimental k-effective Values for $2 P P R-19 A$

\begin{tabular}{|c|c|c|}
\hline & $\begin{array}{c}\text { Almost } \\
\text { Critical Reference } \\
\end{array}$ & Subcritical Reference \\
\hline Date & $1 / 8 / 88$ & $1 / 8 / 88$ \\
\hline Loading & 6 & 4 \\
\hline Reactor Run Number & 8 & 6 \\
\hline Temperature, $\mathrm{K}$ & 301.4 & 300.8 \\
\hline Interface Gap, mila & 53.8 & 54.7 \\
\hline Measured Excess Reactivity, & $-0.43 \pm 0.02$ & $-24.0 \pm 0.1$ \\
\hline Adjustment to $293 \mathrm{~K}, \phi^{\mathrm{b}}$ & $+10.50 \pm 0.92$ & $+9.75 \pm 0.86$ \\
\hline Adjustment to $53.8 \mathrm{mil}, \phi^{\mathrm{C}}$ & $+\infty$ & $+0.08 \pm 0.01$ \\
\hline Adjustment to $1 / 8 / 88, \phi^{\mathrm{d}}$ & $-\infty$ & $m-$ \\
\hline Adjusted Reactivity, $\phi$ & $+10.1 \pm 0.9$ & $-14.7 \pm 0.9$ \\
\hline Experimental K-effective ${ }^{e}$ & $1.000410 \pm 0.000037$ & $0.999403 \pm 0.000037$ \\
\hline
\end{tabular}

a on a scale with arbitrary zero.

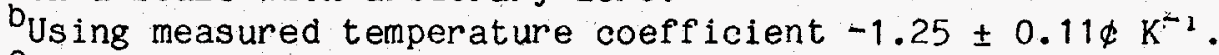

Using measured gap coefficlent $=0.09 \pm 0.01 \notin \mathrm{m}_{11^{-1}}$.

$\mathrm{d}_{\text {Using calculated }}{ }^{24} \mathrm{Pu}$ decay coefficient $-0.02143 \notin$ day $^{-1}$.

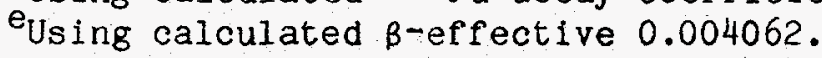


TABLE 10.4. Experimental k-effective Values for ZPPR-19B

Almost

Critical Reference

Date

Loading

Reactor Run Number

Temperature, $\mathrm{K}$

Interface Gap, mil ${ }^{a}$

Measured Excess Reactivity, $\phi$

Adjustment to $293 \mathrm{~K}$, $\phi$

Adjustment to $54.5 \mathrm{mil}, \mathrm{g}^{\mathrm{c}}$

Adjustment to $1 / 29 / 88, \phi^{\mathrm{d}}$

Adjusted Reactivity, $\varnothing$

Experimental K-effective
$1 / 29 / 88$

21

18

299.2

54.5

$13.0 \pm 0.1$

$+7.75 \pm 0.68$

$-\infty$

$-r r$

$+20.8 \pm 0.7$

$1.000804 \pm 0.000027$
Subcritical Reference

$2 / 8 / 88$

28

25

300.5

54.9

$-27.4 \pm 0.2$

$+9.38 \pm 0.83$

$+0.04 \pm 0.004$

$+0.21 \pm 0.02$

$-17.8 \pm 0.8$

$0.999312 \pm 0.000031$

aon a scale with arbitrary zero.

busing measured temperature coefficient $-1.25 \pm 0.11 \notin \mathrm{K}^{-1} \cdot(\mathrm{ZPPR}-18 \mathrm{~A})$

Using measured gap coefficient $-0.09 \pm 0.01 \phi \mathrm{mil}^{-1} \cdot(Z P P R-18 \mathrm{~A})$

$\mathrm{d}_{\text {Using calculated }}{ }^{24} \mathrm{Pu}$ decay coefficient $-0.02143 \notin$ day ${ }^{-1}$.

Using calculated $\beta$-effective 0.003866 . 
TABLE 10.5 Comparison of Criticality Predictions for ZPPR-19A

\begin{tabular}{|c|c|c|c|c|}
\hline \multirow[b]{3}{*}{ Calculation Model ${ }^{a}$} & \multicolumn{4}{|c|}{ Ratio Calculation/Experiment ${ }^{a}$} \\
\hline & \multicolumn{2}{|c|}{$Z P P R-19 A$} & \multicolumn{2}{|c|}{$Z P P R-19 B$} \\
\hline & Critical & Subcritcal & Critical & Subcritical \\
\hline Finite-difference Diffusion & 0.9929 & 0.9929 & 0.9915 & 0.9916 \\
\hline Nodal Diffusion & 0.9906 & $-\infty-\infty$ & 0.9906 & 0.9907 \\
\hline Nodal Transport & 0.9935 & $-m-\infty$ & 0.9926 & 0.9928 \\
\hline
\end{tabular}

${ }^{a}$ Calculations using $x y z$ models with 21 energy groups. 
TABLE 10.6 Delayed Neutron Parameters for ZPPR-19A

\begin{tabular}{|c|c|c|c|}
\hline Family & $a_{1}$ & $\lambda_{i}, \mathbf{s}^{-1}$ & $B_{i}, \%$ \\
\hline 1 & 0.02592 & 0.01291 & 0.01053 \\
\hline 2 & 0.20180 & 0.03157 & 0.08196 \\
\hline 3 & 0.18331 & 0.13080 & 0.07445 \\
\hline 4 & 0.37430 & 0.33809 & 0.15203 \\
\hline 5 & 0.16426 & 1.3798 & 0.06671 \\
\hline 6 & 0.05041 & 3.8157 & 0.02048 \\
\hline
\end{tabular}

B-effective $0.4062 \%$

Prompt neutron lifetime $4.798 \times 10^{-7} \mathrm{~s}$

TABLE 10.7 Delayed Neutron

Parameters for ZPPR -19B

\begin{tabular}{|c|c|c|c|}
\hline Family & $a_{i}$ & $\lambda_{1}, \mathbf{s}^{-1}$ & $B_{i}, \%$ \\
\hline 1 & 0.02501 & 0.01293 & 0.00967 \\
\hline 2 & 0.20238 & 0.03154 & 0.07823 \\
\hline 3 & 0.18339 & 0.13239 & 0.07089 \\
\hline 4. & 0.37061 & 0.34088 & 0.14326 \\
\hline 5 & 0.16635 & 1.3771 & 0.06431 \\
\hline 6 & 0.05226 & 3.8050 & 0.02020 \\
\hline
\end{tabular}


11. SODIUM REACTIVITY WORTH IN ZPPR-19B (R. W. Goin)

In ZPPR Assembly 19B, the reactivity effect of adding (or deleting) sodium was measured using the platercolumn osclilator technique. In this measurement, a $66 \mathrm{~cm}$ long column of $1.27 \mathrm{~cm}$ wide sodium cans was oscillated from fully inserted to $63.5 \mathrm{~cm}$ withdrawn. The reactivity effect of the oscillation was determined using inverse kinetics and correlated with the sodium column position. A least-squares spline-fitting technique was used to obtain the reactivity effect at each $\mathrm{cm}$ of travel, after correcting for reactor drift.

The measurement was then repeated using void cans in place of the sodium column. The result of the void can inverse kinetics output was subtracted from the sodium can oscillation to leave only the reactivity effect of sodium.

This measurement was done in matrix locations $149-40,149-49,155-49$ and 149-58. In 149-58, a $101.6 \mathrm{~cm}$ long column was oscillated, and its stroke was limited to $30.5 \mathrm{~cm}$ from the interface.

Details of the experiment are given in Table 11.1. The material weights for each location are given in Table 11.2.

The integral worths, and differential sodium void worths, were obtained at $1 \mathrm{~cm}$ intervals from the reactor interface. The results are given in Tables 11.3 and 11.4 and in Figs. 11.1 and 11.2 .

The integral worths of the $66 \mathrm{~cm}$ colums were arbitrarily normalized to 0.0 cents at the outermost position of travel. This normalization does not affect the differential worths, but does bias the integral worths. The $101.6 \mathrm{~cm}$ column measurement was normalized to the measurement in 149-40 at $30 \mathrm{~cm}$.

Note that the measured differential void worth is in reality the sum of the sodium removal worth at the interface end of the sodium column, and the sodium addition worth at the reflector end. Because sodium has a positive 
worth above $40 \mathrm{~cm}$, the differential void worths for the $66 \mathrm{~cm}$ long column are too high in the first $\sim 10 \mathrm{~cm}$ from the interface.

Because matrix positions $149-40$ and $149-58$ are in symmetrically equivalent locations, the comparison of the $101.6 \mathrm{~cm}$ long column oscillation in 149-58 with the $66 \mathrm{~cm}$ column in 149-40 provides a measure of the worth of adding sodium at the reflector end of the $66 \mathrm{~cm}$ column as it is withdrawn. The result of subtracting the $101.6 \mathrm{~cm}$ column measurement (in location 149-58) is shown in Figure 11.3.

This measurement provides two corrections to the $66 \mathrm{~cm}$ sodium column measurements. First, by plotting the worth above $66 \mathrm{~cm}$ and the uncorrected sodium worth on the same curve, the non-zero offset correction is obtained which gives the best overlap. This correction is $0.0085 \phi \pm 0.0015 \phi$, and accounts for sodium still having a non-zero positive worth (as shown in Figure 11.3) when it is withdrawn $650 \mathrm{~mm}$.

The second correction is to the point-by-point integral worth. When the $66 \mathrm{~cm}$ column is moved from the interface to $1 \mathrm{~mm}$ back, sodium displaces void in the space $660 \mathrm{~mm}$ to $661 \mathrm{~mm}$, etc. The integral sodium worths including both corrections are shown in Figure 11.3.

An attempt was made to extract a simflar correction to the differential worth. The differential vold worth shown in Figure 11.2 contains a number of irregularities, particularly in location 149-49. The source of the dips and peaks in the differential worth is not presently known.

The result of correcting for the end effect is shown for the location 149-40 data in Figure 11.4. While the magnitude of the correction may be uncertain, it is clear that the void reactivity coefficient has a smaller positive value near the interface than the measurements using $66 \mathrm{~cm}$ long sodium columns would indicate.

These corrections were only done for location $149-40$ because it is symmetric with the long column measurement. But it does indicate that the 
measured sodium voiding reactivity effect per unit mass of sodium has a substantial correction of about $29 \%$ at the interface.

The uncertainties presented for the integral column worths only represent statistical uncertainties; those presented for the differential worths were estimated by $\sqrt{2}$ times the statistical uncertainty of the integral worth. 
TABLE 11.1 Details of Sodium Void Worth Measurements in ZPPR 19B

\begin{tabular}{|c|c|c|c|c|c|}
\hline Location & Description & $\begin{array}{l}\text { Drawer } \\
\text { Master }\end{array}$ & $\begin{array}{l}\text { Reactor Run } \\
\text { No. } \\
\end{array}$ & $\begin{array}{l}\text { Loading } \\
\text { No. }\end{array}$ & Date \\
\hline $149-40$ & Sodium & $19-0-835$ & 22 & 25 & $2 / 3 / 88$ \\
\hline $149-40$ & Void & $19-0-836$ & 23 & 26 & $2 / 4 / 88$ \\
\hline $149-49$ & Sodium & $19 \div 0 \div 835$ & 22 & 25 & $2 / 3 / 88$ \\
\hline $149-49$ & Void & $19-0-836$ & 23 & 26 & $2 / 4 / 88$ \\
\hline $155 \div 49$ & Sodium & $19 * 0-839$ & 24 & 27 & $2 / 5 / 88$ \\
\hline $155-49$ & Void & $19-0-840$ & 23 & 26 & $2 / 4 / 88$ \\
\hline $149-58$ & Sodium & $19=0-837$ & 22 & 25 & $2 / 3 / 88$ \\
\hline $149-58$ & Void & $19-0-838$ & 23 & 26 & $2 / 4 / 88$ \\
\hline
\end{tabular}


TABLE 11.2. Details of Sodium Cans and Void Cans

\begin{tabular}{|c|c|c|c|c|c|c|}
\hline \multirow[b]{2}{*}{$\begin{array}{c}\text { Axial } \\
\text { Sequence } \\
\end{array}$} & \multirow[b]{2}{*}{$\begin{array}{l}\text { Length } \\
\text { in. } \\
\end{array}$} & \multicolumn{4}{|c|}{ Sodium Cans } & \multirow{2}{*}{$\begin{array}{l}\text { Void Cans } \\
\text { Can Mass } \\
\mathrm{g} \\
\end{array}$} \\
\hline & & $\begin{array}{c}\text { Serial } \\
\text { No. } \\
\end{array}$ & $\begin{array}{l}\text { Total Mass } \\
\mathrm{g} \\
\end{array}$ & $\begin{array}{c}\text { Can Mass } \\
8 \\
\end{array}$ & $\begin{array}{l}\text { Na Mass } \\
\mathrm{g} \\
\end{array}$ & \\
\hline & & & $149-40$ & & & \\
\hline $\begin{array}{l}1 \\
2 \\
3 \\
4\end{array}$ & $\begin{array}{l}6 \\
7 \\
7 \\
6\end{array}$ & $\begin{array}{l}106581 \\
33672 \\
12426 \\
106151\end{array}$ & $\begin{array}{l}143.65 \\
167.51 \\
166.58 \\
144.24\end{array}$ & $\begin{array}{l}61.22 \\
70.84 \\
70.15 \\
61.70\end{array}$ & $\begin{array}{l}82.43 \\
96.67 \\
96.43 \\
82.54\end{array}$ & $\begin{array}{l}61.55 \\
70.94 \\
71.30 \\
61.90\end{array}$ \\
\hline & & & $149-49$ & & & \\
\hline $\begin{array}{l}1 \\
2 \\
3 \\
4\end{array}$ & $\begin{array}{l}6 \\
7 \\
7 \\
6\end{array}$ & $\begin{array}{r}106605 \\
34022 \\
12330 \\
110779\end{array}$ & $\begin{array}{l}145.19 \\
168.25 \\
167.68 \\
143.04\end{array}$ & $\begin{array}{l}62.88 \\
70.74 \\
70.75 \\
60.13\end{array}$ & $\begin{array}{l}82.31 \\
97.51 \\
96.93 \\
82.91\end{array}$ & $\begin{array}{l}62.26 \\
70.76 \\
70.91 \\
61.32\end{array}$ \\
\hline & & & $155-49$ & & & \\
\hline $\begin{array}{l}1 \\
2 \\
3 \\
4\end{array}$ & $\begin{array}{l}6 \\
7 \\
7 \\
6\end{array}$ & $\begin{array}{r}105965 \\
107991 \\
33671 \\
11059\end{array}$ & $\begin{array}{l}144.63 \\
167.99 \\
166.70 \\
146.05\end{array}$ & $\begin{array}{l}61.63 \\
70.57 \\
69.99 \\
62.89\end{array}$ & $\begin{array}{l}83.00 \\
97.42 \\
96.71 \\
83.16\end{array}$ & $\begin{array}{l}61.89 \\
70.50 \\
69.61 \\
62.29\end{array}$ \\
\hline & & & $149-58$ & & & \\
\hline $\begin{array}{l}1 \\
2 \\
3 \\
4 \\
5 \\
6\end{array}$ & $\begin{array}{l}8 \\
8 \\
4 \\
8 \\
8 \\
4\end{array}$ & $\begin{array}{l}23732 \\
23726 \\
04030 \\
24213 \\
24926 \\
04771\end{array}$ & $\begin{array}{r}189.55 \\
191.95 \\
98.23 \\
190.05 \\
189.06 \\
98.15\end{array}$ & $\begin{array}{l}79.21 \\
80.39 \\
43.39 \\
79.21 \\
80.74 \\
43.64\end{array}$ & $\begin{array}{r}110.34 \\
111.56 \\
54.84 \\
110.84 \\
108.32 \\
54.51\end{array}$ & $\begin{array}{l}79.34 \\
80.28 \\
43.04 \\
79.25 \\
80.79 \\
43.66\end{array}$ \\
\hline
\end{tabular}


TABLE 11.3 Integral Reactivity Worth, in Cents, of Axial Sodium Column, ZPPR 19B

\begin{tabular}{|c|c|c|c|c|}
\hline $\begin{array}{l}\text { Distance from } \\
\text { Interface (mm) }\end{array}$ & $149-40$ & $149-49$ & $149-58,305 \mathrm{~mm}$ Stroke & $155-49$ \\
\hline 2.31 & $-0.05594 \pm 0.00055$ & $-0.06531 \pm 0.00053$ & $-0.04718 \pm 0.00050$ & $-0.06551+0.00042$ \\
\hline 10.00 & $-0.05159 \pm 0.00051$ & $-0.06150 \pm 0.00049$ & $-0.04477 \pm 0.00046$ & $-0.06088 \pm 0.00039$ \\
\hline 20.00 & $-0.04682 \pm 0.00057$ & $-0.05691 \pm 0.00055$ & $-0.04177 \pm 0.00051$ & $-0.05650 \pm 0.00044$ \\
\hline 30.00 & $-0.04300 \pm 0.00050$ & $-0.05270 \pm 0.00048$ & $-0.03891 \pm 0.00046$ & $-0.05390 \pm 0.00038$ \\
\hline 40.00 & $-0.03934 \pm 0.00055$ & $-0.04816 \pm 0.00053$ & $-0.03583 \pm 0.00049$ & $-0.05091 \pm 0.00042$ \\
\hline 50.00 & $-0.03547 \pm 0.00050$ & $-0.04300 \pm 0.00049$ & $-0.03241 \pm 0.00046$ & $-0.04655+0.00039$ \\
\hline 60.00 & $-0.03152 \pm 0.00052$ & $-0.03766 \pm 0.00051$ & $-0.02872 \pm 0.00048$ & $-0.04141 \pm 0.00040$ \\
\hline 70.00 & $-0.02785 \pm 0.00053$ & $-0.03348 \pm 0.00052$ & $-0.02502 \pm 0.00048$ & $-0.03728 \pm 0.00041$ \\
\hline 80.00 & $-0.02449 \pm 0.00049$ & $-0.03052 \pm 0.00047$ & $-0.02134 \pm 0.00046$ & $-0.03428 \pm 0.00038$ \\
\hline 90.00 & $-0.02124 \pm 0.00054$ & $-0.02707 \pm 0.00052$ & $-0.01795 \pm 0.00049$ & $-0.03107 \pm 0.00042$ \\
\hline 100.00 & $-0.01796 \pm 0.00050$ & $-0.02216 \pm 0.00049$ & $=0.01501 \pm 0.00046$ & $-0.02695 \pm 0.00039$ \\
\hline 110.00 & $-0.01466 \pm 0.00052$ & $-0.01632 \pm 0.00050$ & $-0.01240 \pm 0.00047$ & $-0.02224 \pm 0.00040$ \\
\hline 120.00 & $-0.01125 \pm 0.00054$ & $-0.01175 \pm 0.00052$ & $-0.00967 \pm 0.00049$ & $-0.01831 \pm 0.00041$ \\
\hline 130.00 & $-0.00772 \pm 0.00049$ & $-0.00868 \pm 0.00047$ & $-0.00675 \pm 0.00045$ & $-0.01529 \pm 0.00037$ \\
\hline 140.00 & $-0.00409 \pm 0.00054$ & $-0.00596 \pm 0.00051$ & $-0.00396 \pm 0.00049$ & $-0.01242 \pm 0.00041$ \\
\hline 150.00 & $-0.00041 \pm 0.00051$ & $-0.00274 \pm 0.00048$ & $-0.00151 \pm 0.00046$ & $-0.00908+0.00039$ \\
\hline 160.00 & $0.00320 \pm 0.00051$ & $0.00090 \pm 0.00048$ & $0.00072 \pm 0.00047$ & $-0.00546 \pm 0.00039$ \\
\hline 170.00 & $0.00602 \pm 0.00054$ & $0.00446 \pm 0.00051$ & $0.00332 \pm 0.00049$ & $-0.00238 \pm 0.00041$ \\
\hline 180.00 & $0.00793 \pm 0.00049$ & $0.00786 \pm 0.00046$ & $0.00637 \pm 0.00045$ & $0.00003 \pm 0.00037$ \\
\hline 190.00 & $0.00959 \pm 0.00053$ & $0.01103 \pm 0.00051$ & $0.00953 \pm 0.00049$ & $0.00224 \pm 0.00041$ \\
\hline 200.00 & $0.01168 \pm 0.00051$ & $0.01386 \pm 0.00049$ & $0.01239 \pm 0.00047$ & $0.00477 \pm 0.00039$ \\
\hline 210.00 & $0.01411 \pm 0.00050$ & $0.01637 \pm 0.00048$ & $0.01494 \pm 0.00046$ & $0.00758 \pm 0.00039$ \\
\hline 220.00 & $0.01640 \pm 0.00053$ & $0.01889 \pm 0.00051$ & $0.01713 \pm 0.00049$ & $0.01053 \pm 0.00041$ \\
\hline 230.00 & $0.01845 \pm 0.00048$ & $0.02144 \pm 0.00046$ & $0.01896 \pm 0.00045$ & $0.01358 \pm 0.00037$ \\
\hline 240.00 & $0.02033 \pm 0.00053$ & $0.02371 \pm 0.00051$ & $0.02052 \pm 0.00049$ & $0.01635 \pm 0.00041$ \\
\hline 250.00 & $0.02213 \pm 0.00051$ & $0.02516 \pm 0.00049$ & $0.02195 \pm 0.00047$ & $0.01827 \pm 0.00040$ \\
\hline 260.00 & $0.02384 \pm 0.00050$ & $0.02595 \pm 0.00047$ & $0.02329 \pm 0.00047$ & $0.01941 \pm 0.00038$ \\
\hline 270.00 & $0.02516 \pm 0.00053$ & $0.02736 \pm 0.00051$ & $0.02463 \pm 0.00049$ & $0.02056 \pm 0.00042$ \\
\hline 280.00 & $0.02602 \pm 0.00049$ & $0.02976 \pm 0.00046$ & $0.02603 \pm 0.00046$ & $0.02191 \pm 0.00038$ \\
\hline 290.00 & $0.02675 \pm 0.00052$ & $0.03247 \pm 0.00050$ & $0.02725 \pm 0.00052$ & $0.02341 \pm 0.00041$ \\
\hline 300.00 & $0.02794 \pm 0.00052$ & $0.03417 \pm 0.00050$ & $0.02794 \pm 0.00052$ & $0.02494 \pm 0.00040$ \\
\hline 310.00 & $0.02956 \pm 0.00049$ & $0.03489 \pm 0.00047$ & $-\infty-$ & $0.02644 \pm 0.00038$ \\
\hline 320.00 & $0.03077 \pm 0.00053$ & $0.03520 \pm 0.00051$ & 每 & $0.02735 \pm 0.00042$ \\
\hline 330.00 & $0.03126 \pm 0.00049$ & $0.03534 \pm 0.00047$ & $m-m$ & $0.02747 \pm 0.00038$ \\
\hline 340.00 & $0.03121 \pm 0.00052$ & $0.03535 \pm 0.00050$ & $m$ & $0.02712 \pm 0.00040$ \\
\hline 350.00 & $0.03098 \pm 0.00052$ & $0.03537 \pm 0.00050$ & $m-$ & $0.02716 \pm 0.00040$ \\
\hline 360.00 & $0.03062 \pm 0.00049$ & $0.03539 \pm 0.00047$ & $-m-$ & $0.02762 \pm 0.00038$ \\
\hline 370.00 & $0.03044 \pm 0.00053$ & $0.03533 \pm 0.00051$ & $-\infty$ & $0.02786+0.00041$ \\
\hline 380.00 & $0.03059+0.00049$ & $003516+0$ 000117 & & \\
\hline
\end{tabular}




\begin{tabular}{|c|c|c|c|c|}
\hline terface $(\mathrm{mm})$ & $149-40$ & $149-49$ & $149-58,305 \mathrm{~mm}$ Stroke & $155-49$ \\
\hline 390.00 & $0.03083 \pm 0.00051$ & $0.03490 \pm 0.00049$ & $m-m$ & $0.02694 \pm 0.00040$ \\
\hline 400.00 & $0.03043 \pm 0.00053$ & $0.03462 \pm 0.00050$ & $-n$ & $0.02648 \pm 0.00041$ \\
\hline 410.00 & $0.02935 \pm 0.00048$ & $0.03433 \pm 0.00046$ & $\therefore-m$ & $0.02623 \pm 0.00037$ \\
\hline 420.00 & $0.02835 \pm 0.00053$ & $0.03343 \pm 0.00051$ & $-\cdots$ & $0.02591 \pm 0.00041$ \\
\hline 430.00 & $0.02793 \pm 0.00050$ & $0.03153 \pm 0.00048$ & $\rightarrow-$ & $0.02535 \pm 0.00039$ \\
\hline 440.00 & $0.02786 \pm 0.00051$ & $0.02909 \pm 0.00049$ & $-m$ & $0.02454 \pm 0.00040$ \\
\hline 450.00 & $0.02727 \pm 0.00053$ & $0.02788 \pm 0.00051$ & -- & $0.02351 \pm 0.00041$ \\
\hline 460.01 & $0.02606 \pm 0.00048$ & $0.02812 \pm 0.00046$ & --- & $0.02227 \pm 0.00037$ \\
\hline 470.01 & $0.02443 \pm 0.00053$ & $0.02828 \pm 0.00051$ & $-\rightarrow$ & $0.02104 \pm 0.00041$ \\
\hline 480.01 & $0.02252 \pm 0.00050$ & $0.02710 \pm 0.00048$ & $-m$ & $0.02005 \pm 0.00039$ \\
\hline 490.01 & $0.02043 \pm 0.00050$ & $0.02470 \pm 0.00048$ & $-r$ & $0.01914 \pm 0.00039$ \\
\hline 500.01 & $0.01872 \pm 0.00053$ & $0.02175 \pm 0.00051$ & --- & $0.01752 \pm 0.00041$ \\
\hline 510.01 & $0.01749 \pm 0.00048$ & $0.01837 \pm 0.00046$ & $-\infty$ & $0.01506 \pm 0.00037$ \\
\hline 520.01 & $0.01629 \pm 0.00053$ & $0.01518 \pm 0.00051$ & $-\cdots$ & $0.01251 \pm 0.00041$ \\
\hline 530.01 & $0.01464 \pm 0.00051$ & $0.01288 \pm 0.00049$ & $n-$ & $0.01072 \pm 0.00039$ \\
\hline 540.01 & $0.01261 \pm 0.00050$ & $0.01137 \pm 0.00048$ & --- & $0.00963 \pm 0.00039$ \\
\hline 550.01 & $0.01071 \pm 0.00053$ & $0.00969 \pm 0.00051$ & $-m$ & $0.00874 \pm 0.00041$ \\
\hline 560.01 & $0.00904 \pm 0.00048$ & $0.00762 \pm 0.00046$ & $m$ & $0.00791 \pm 0.00037$ \\
\hline 570.01 & $0.00757 \pm 0.00053$ & $0.00555 \pm 0.00051$ & $--\Rightarrow$ & $0.00706 \pm 0.00041$ \\
\hline 580.01 & $0.00622 \pm 0.00051$ & $0.00400 \pm 0.00049$ & $-\infty$ & $0.00607 \pm 0.00040$ \\
\hline 590.01 & $0.00496 \pm 0.00050$ & $0.00297 \pm 0.00048$ & $m r$ & $0.00493 \pm 0.00039$ \\
\hline 600.01 & $0.00390 \pm 0.00054$ & $0.00212 \pm 0.00052$ & $\cdots$ & $0.00375 \pm 0.00042$ \\
\hline 610.01 & $0.00305 \pm 0.00049$ & $0.00134 \pm 0.00047$ & $=-\infty$ & $0.00257 \pm 0.00038$ \\
\hline 620.01 & $0.00230 \pm 0.00054$ & $0.00070 \pm 0.00052$ & -.- & $0.00156 \pm 0.00042$ \\
\hline 630.01 & $0.00143 \pm 0.00052$ & $0.00025 \pm 0.00050$ & -- & $0.00099 \pm 0.00040$ \\
\hline 640.01 & $0.00046 \pm 0.00057$ & $0.00000 \pm 0.00055$ & $-\cdots$ & $0.00082 \pm 0.00044$ \\
\hline 650.01 & $0.00000 \pm 0.00053$ & $0.00000 \pm 0.00051$ & $\ddot{m}$ & $0.00000 \pm 0.00041$ \\
\hline
\end{tabular}




\begin{tabular}{|c|c|c|c|c|}
\hline $\begin{array}{l}\text { Distance from } \\
\text { Interface (mm) }\end{array}$ & $149-40$ & $149-49$ & $149-58,305 \mathrm{~m}$ & $155-49$ \\
\hline 2.31 & $1.1123 \pm 0.0381$ & $0.9415 \pm 0.0373$ & $0.5883 \pm 0.0310$ & $1.2412 \pm 0.0293$ \\
\hline 10.00 & $0.9719 \pm 0.0395$ & $0.8841 \pm 0.0388$ & $0.5669 \pm 0.0320$ & $0.9776 \pm 0.0300$ \\
\hline 20.00 & $0.7892 \pm 0.0387$ & $0.8093 \pm 0.0381$ & $0.5391 \pm 0.0315$ & $0.6347 \pm 0.0293$ \\
\hline 30.00 & $0.6568 \pm 0.0371$ & $0.7781 \pm 0.0365$ & $0.5327 \pm 0.0301$ & $0.4279 \pm 0.0281$ \\
\hline 40.00 & $0.6944 \pm 0.0374$ & $0.8934 \pm 0.0368$ & $0.5984 \pm 0.0303$ & $0.6789 \pm 0.0283$ \\
\hline 50.00 & $0.7318 \pm 0.0355$ & $1.0087 \pm 0.0350$ & $0.6641 \pm 0.0289$ & $0.9298 \pm 0.0269$ \\
\hline 60.00 & $0.7043 \pm 0.0377$ & $0.8858 \pm 0.0371$ & $0.6828 \pm 0.0306$ & $0.8642 \pm 0.0285$ \\
\hline 70.00 & $0.6473 \pm 0.0354$ & $0.6554 \pm 0.0348$ & $0.6804 \pm 0.0286$ & $0.6556 \pm 0.0267$ \\
\hline 80.00 & $0.5990 \pm 0.0360$ & $0.4997 \pm 0.0354$ & $0.6659 \pm 0.0291$ & $0.5035 \pm 0.0272$ \\
\hline 90.00 & $0.6013 \pm 0.0369$ & $0.7707 \pm 0.0363$ & $0.5834 \pm 0.0299$ & $0.6745 \pm 0.0279$ \\
\hline 100.00 & $0.6036 \pm 0.0350$ & $1.0418 \pm 0.0345$ & $0.5010 \pm 0.0284$ & $0.8455 \pm 0.0265$ \\
\hline 110.00 & $0.6181 \pm 0.0374$ & $0.9798 \pm 0.0368$ & $0.4883 \pm 0.0303$ & $0.8094 \pm 0.0283$ \\
\hline 120.00 & $0.6404 \pm 0.0353$ & $0.7039 \pm 0.0345$ & $0.5206 \pm 0.0286$ & $0.6403 \pm 0.0267$ \\
\hline 130.00 & $0.6620 \pm 0.0357$ & $0.4535 \pm 0.0341$ & $0.5463 \pm 0.0289$ & $0.4887 \pm 0.0269$ \\
\hline 140.00 & $0.6733 \pm 0.0371$ & $0.5469 \pm 0.0352$ & $0.4835 \pm 0.0301$ & $0.5721 \pm 0.0281$ \\
\hline 150.00 & $0.6847 \pm 0.0350$ & $0.6404 \pm 0.0332$ & $0.4208 \pm 0.0284$ & $0.6555 \pm 0.0264$ \\
\hline 160.00 & $0.6022 \pm 0.0372$ & $0.6698 \pm 0.0352$ & $0.4363 \pm 0.0302$ & $0.6295 \pm 0.0281$ \\
\hline 170.00 & $0.4363 \pm 0.0354$ & $0.6423 \pm 0.0336$ & $0.5215 \pm 0.0288$ & $0.5058 \pm 0.0269$ \\
\hline 180.00 & $0.2705 \pm 0.0350$ & $0.6147 \pm 0.0335$ & $0.6068 \pm 0.0288$ & $0.3821 \pm 0.0268$ \\
\hline 190.00 & $0.3449 \pm 0.0368$ & $0.5517 \pm 0.0352$ & $0.5537 \pm 0.0302$ & $0.4369 \pm 0.0282$ \\
\hline 200.00 & $0.4220 \pm 0.0346$ & $0.4883 \pm 0.0330$ & $0.4992 \pm 0.0284$ & $0.4936 \pm 0.0265$ \\
\hline 210.00 & $0.4444 \pm 0.0365$ & $0.4578 \pm 0.0349$ & $0.4386 \pm 0.0301$ & $0.5337 \pm 0.0279$ \\
\hline 220.00 & $0.3998 \pm 0.0353$ & $0.4677 \pm 0.0337$ & $0.3705 \pm 0.0291$ & $0.5534 \pm 0.0269$ \\
\hline 230.00 & $0.3552 \pm 0.0348$ & $0.4775 \pm 0.0333$ & $0.3024 \pm 0.0288$ & $0.5731 \pm 0.0267$ \\
\hline 240.00 & $0.3393 \pm 0.0369$ & $0.3435 \pm 0.0354$ & $0.2762 \pm 0.0305$ & $0.4323 \pm 0.0283$ \\
\hline 250.00 & $0.3263 \pm 0.0345$ & $0.1949 \pm 0.0330$ & $0.2543 \pm 0.0291$ & $0.2756 \pm 0.0264$ \\
\hline 260.00 & $0.2867 \pm 0.0363$ & $0.1688 \pm 0.0347$ & $0.2436 \pm 0.0308$ & $0.1911 \pm 0.0278$ \\
\hline 270.00 & $0.2017 \pm 0.0356$ & $0.3517 \pm 0.0340$ & $0.2521 \pm 0.0306$ & $0.2302 \pm 0.0272$ \\
\hline 280.00 & $0.1167 \pm 0.0346$ & $0.5346 \pm 0.0332$ & $0.2608 \pm 0.0331$ & $0.2692 \pm 0.0266$ \\
\hline 290.00 & $0.1752 \pm 0.0369$ & $0.4090 \pm 0.0354$ & $0.1766 \pm 0.0372$ & $0.2790 \pm 0.0283$ \\
\hline 300.00 & $0.2634 \pm 0.0345$ & $0.2199 \pm 0.0330$ & $0.0734 \pm 0.0372$ & $0.2828 \pm 0.0264$ \\
\hline 310.00 & $0.2881 \pm 0.0360$ & $0.0757 \pm 0.0344$ & - & $0.2425 \pm 0.0276$ \\
\hline 320.00 & $0.1567 \pm 0.0358$ & $0.0420 \pm 0.0343$ & $m$ & $0.0943 \pm 0.0274$ \\
\hline 330.00 & $0.0254 \pm 0.0345$ & $0.0084 \pm 0.0331$ &.-- & $-0.0540 \pm 0.0264$ \\
\hline 340.00 & $-0.0276 \pm 0.0369$ & $0.0015 \pm 0.0354$ & $-m$ & $-0.0314 \pm 0.0283$ \\
\hline 350.00 & $-0.0544 \pm 0.0346$ & $0.0036 \pm 0.0332$ & $-\cdots$ & $0.0481 \pm 0.0264$ \\
\hline 360.00 & $-0.0631 \pm 0.0356$ & $0.0007 \pm 0.0342$ & $-\cdots$ & $0.0911 \pm 0.0272$ \\
\hline 370.00 & $-0.0032 \pm 0.0361$ & $-0.0211 \pm 0.0346$ & $-\cdots$ & $-0.0042 \pm 0.0276$ \\
\hline
\end{tabular}


TABLE 11.4 (Contd)

Distance from

Interface (mm)

380.00

390.00

400.00

410.00

420.00

430.00

440.00

450.00

460.01

470.01

480.01

490.01

500.01

510.01

520.01

530.01

540.01

550.01

560.01

570.01

580.01

590.01

600.01

610.01

620.01

630.01

640.01

650.01

\begin{abstract}
$149-40$
\end{abstract}
$0.0565 \pm 0.0345$ $-0.0094 \pm 0.0369$

$-0.1373 \pm 0.0346$

$-0.2352 \pm 0.0353$

$-0.1313 \pm 0.0363$

$-0.0275 \pm 0.0345$

$-0.0516 \pm 0.0368$

$-0.1650 \pm 0.0348$

$-0.2753 \pm 0.0351$

$-0.3267 \pm 0.0366$

$-0.3782 \pm 0.0345$

$-0.3587 \pm 0.0366$

$-0.2708 \pm 0.0350$

$-0.1831 \pm 0.0348$

$=0.2616 \pm 0.0368$

$-0.3453 \pm 0.0345$

$-0.3737 \pm 0.0364$

$-0.3285 \pm 0.0353$

$-0.2833 \pm 0.0348$

$-0.2607 \pm 0.0369$

$-0.2409 \pm 0.0346$

$-0.2146 \pm 0.0365$

$-0.1762 \pm 0.0358$

$-0.1379 \pm 0.0361$

$-0.1493 \pm 0.0382$

$-0.1724 \pm 0.0405$

$-0.1525 \pm 0.0415$

$-0.0160 \pm 0.0415$

\begin{abstract}
$149 \div 49$
\end{abstract}
$-0.0428 \pm 0.0331$

$-0.0505 \pm 0.0354$

$-0.0513 \pm 0.0333$

$-0.0754 \pm 0.0339$

$-0.2571 \pm 0.0349$

$-0.4388 \pm 0.0330$

$-0.3560 \pm 0.0353$

$-0.0889+0.0334$

$0.1529 \pm 0.0336$

$-0.0942 \pm 0.0351$

$-0.3413 \pm 0.0330$

$-0.5032 \pm 0.0352$

$-0.5832 \pm 0.0336$

$-0.6631 \pm 0.0335$

$-0.5053 \pm 0.0353$

$-0.3401+0.0330$

$-0.2756 \pm 0.0349$

$-0.3450 \pm 0.0339$

$-0.4146 \pm 0.0333$

$-0.3342 \pm 0.0354$

$-0.2352 \pm 0.0333$

$-0.1653 \pm 0.0349$

$-0.1494 \pm 0.0344$

$-0.1336 \pm 0.0347$

$-0.1013 \pm 0.0366$

$-0.0650 \pm 0.0388$

$-0.0247 \pm 0.0398$

$0.0264 \pm 0.0398$
$149-58,305 \mathrm{~m}$

$155-49$

\begin{tabular}{|c|c|}
\hline & $-0.0996 \pm 0.0264$ \\
\hline & $-0.1046 \pm 0.0282$ \\
\hline & $-0.0652 \pm 0.0265$ \\
\hline & $=0.0366 \pm 0.0270$ \\
\hline & $=0.0814 \pm 0.0278$ \\
\hline & $-0.1261 \pm 0.0264$ \\
\hline & $-0.1687 \pm 0.0281$ \\
\hline & $-0.2095 \pm 0.0266$ \\
\hline & $-0.2463 \pm 0.0269$ \\
\hline & $-0.2046 \pm 0.0280$ \\
\hline & $-0.1629 \pm 0.0264$ \\
\hline & $-0.2212 \pm 0.0281$ \\
\hline & $-0.3760 \pm 0.0269$ \\
\hline & $-0.5308 \pm 0.0267$ \\
\hline & $-0.3995 \pm 0.0281$ \\
\hline & $-0.2591 \pm 0.0264$ \\
\hline & $-0.1732 \pm 0.0279$ \\
\hline & $-0.1595 \pm 0.0270$ \\
\hline & $-0.1459 \pm 0.0267$ \\
\hline & $-0.1696 \pm 0.0283$ \\
\hline & $-0.1980 \pm 0.0266$ \\
\hline & $-0.2164 \pm 0.0280$ \\
\hline & $-0.2163 \pm 0.0276$ \\
\hline & $-0.2162 \pm 0.0277$ \\
\hline & $-0.1474 \pm 0.0294$ \\
\hline & $-0.0622 \pm 0.0311$ \\
\hline & $-0.0533 \pm 0.0319$ \\
\hline & $-0.2509 \pm 0.0319$ \\
\hline
\end{tabular}


TABLE 11.5 Integral Reactivity worth, in Cents, of Sodium Column in 149-40

Distance from

Interface (mm)

2.31

10.00

20.00

30.00

40.00

50.00

60.00

70.00

80.00

90.00

100.00

110.00

120.00

130.00

140.00

150.00

160.00

170.00

180.00

190.00

200.00

210.00

220.00

230.00

240.00

250.00

260.00

270.00

280.00

290.00

300.00

310.00

320.00

330.00

340.00

350.00

360.00

370.00

380.00

390.00

400.00

410.00

420.00

430.00

440.00

450.00

460.01

470.01

480.01

490.01
Corrected, Adjusted

$-0.03868 \pm 0.00183$

$-0.03627 \pm 0.00180$

$-0.03327 \pm 0.00185$

$-0.03041 \pm 0.00179$

$-0.02733 \pm 0.00183$

$-0.02391 \pm 0.00179$

$-0.02022 \pm 0.00181$

$-0.01652 \pm 0.00182$

$-0.01284 \pm 0.00179$

$-0.00945 \pm 0.00182$

$-0.00651 \pm 0.00179$

$-0.00390 \pm 0.00181$

$-0.00117 \pm 0.00182$

$0.00175 \pm 0.00179$

$0.00454 \pm 0.00182$

$0.00699 \pm 0.00180$

$0.00922 \pm 0.00180$

$0.01182 \pm 0.00182$

$0.01487 \pm 0.00179$

$0.01803 \pm 0.00182$

$0.02089 \pm 0.00180$

$0.02344 \pm 0.00179$

$0.02563 \pm 0.00182$

$0.02746 \pm 0.00178$

$0.02902 \pm 0.00182$

$0.03045 \pm 0.00180$

$0.03179 \pm 0.00180$

$0.03313 \pm 0.00182$

$0.03453 \pm 0.00179$

$0.03575 \pm 0.00182$

$0.03644 \pm 0.00182$

$0.03806 \pm 0.00166$

$0.03927 \pm 0.00168$

$0.03976 \pm 0.00166$

$0.03971 \pm 0.00167$

$0.03948 \pm 0.00167$

$0.03912 \pm 0.00166$

$0.03894 \pm 0.00168$

$0.03909 \pm 0.00166$

$0.03933 \pm 0.00167$

$0.03893 \pm 0.00168$

$0.03785 \pm 0.00166$

$0.03685 \pm 0.00168$

$0.03642 \pm 0.00167$

$0.03636 \pm 0.00167$

$0.03577 \pm 0.00168$

$0.03456 \pm 0.00166$

$0.03293 \pm 0.00168$

$0.03102 \pm 0.00167$

$0.02893 \pm 0.00167$
Worth Above

$660 \mathrm{~mm}$

$0.00876 \pm 0.00072$

$0.00682 \pm 0.00067$

$0.00505 \pm 0.00075$

$0.00409 \pm 0.00066$

$0.00351 \pm 0.00072$

$0.00306 \pm 0.00066$

$0.00280 \pm 0.00068$

$0.00283 \pm 0.00070$

$0.00315 \pm 0.00065$

$0.00329 \pm 0.00071$

$0.00295 \pm 0.00066$

$0.00226 \pm 0.00068$

$0.00158 \pm 0.00071$

$0.00097 \pm 0.00065$

$0.00013 \pm 0.00071$

$-0.00110 \pm 0.00067$

$-0.00248 \pm 0.00067$

$-0.00270 \pm 0.00071$

$-0.00156 \pm 0.00065$

$-0.00006 \pm 0.00070$

$0.00071 \pm 0.00067$

$0.00083 \pm 0.00066$

$0.00073 \pm 0.00070$

$0.00051 \pm 0.00064$

$0.00019 \pm 0.00070$

$-0.00018 \pm 0.00068$

$-0.00055 \pm 0.00067$

$-0.00053 \pm 0.00071$

$0.00001 \pm 0.00065$

$0.00050 \pm 0.00072$

$0.00000 \pm 0.00072$

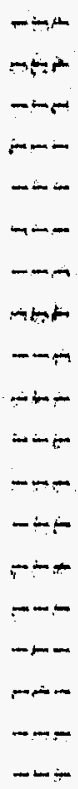

Uncorrected

$-0.05594 \pm 0.00055$

$-0.05159 \pm 0.00051$

$-0.04682 \pm 0.00057$

$-0.04300 \pm 0.00050$

$-0.03934 \pm 0.00055$

$-0.03547 \pm 0.00050$

$-0.03152 \pm 0.00052$

$-0.02785 \pm 0.00053$

$-0.02449 \pm 0.00049$

$-0.02124 \pm 0.00054$

$-0.01796 \pm 0.00050$

$-0.01466 \pm 0.00052$

$-0.01125 \pm 0.00054$

$-0.00772 \pm 0.00049$

$-0.00409 \pm 0.00054$

$-0.00041 \pm 0.00051$

$0.00320 \pm 0.00051$

$0.00602 \pm 0.00054$

$0.00793 \pm 0.00049$

$0.00959 \pm 0.00053$

$0.01168 \pm 0.00051$

$0.01411 \pm 0.00050$

$0.01640 \pm 0.00053$

$0.01845 \pm 0.00048$

$0.02033 \pm 0.00053$

$0.02213 \pm 0.00051$

$0.02384 \pm 0.00050$

$0.02516 \pm 0.00053$

$0.02602 \pm 0.00049$

$0.02675 \pm 0.00052$

$0.02794 \pm 0.00052$

$0.02956 \pm 0.00049$

$0.03077 \pm 0.00053$

$0.03126 \pm 0.00049$

$0.03121 \pm 0.00052$

$0.03098 \pm 0.00052$

$0.03062 \pm 0.00049$

$0.03044 \pm 0.00053$

$0.03059 \pm 0.00049$

$0.03083 \pm 0.00051$

$0.03043 \pm 0.00053$

$0.02935 \pm 0.00048$

$0.02835 \pm 0.00053$

$0.02792 \pm 0.00050$

$0.02786 \pm 0.00051$

$0.02727 \pm 0.00053$

$0.02606 \pm 0.00048$

$0.02443 \pm 0.00053$

$0.02252 \pm 0.00050$

$0.02043 \pm 0.00050$ 


\begin{tabular}{|c|c|c|c|}
\hline $\begin{array}{l}\text { Distance from } \\
\text { Interface (mm) }\end{array}$ & $\begin{array}{c}\text { Corrected, } \\
\text { Adjusted } \\
\end{array}$ & $\begin{array}{c}\text { Worth Above } \\
660 \mathrm{~mm} .\end{array}$ & Uncorrected \\
\hline 500.01 & $0.02722 \pm 0.00168$ & --- & $0.01872 \pm 0.00053$ \\
\hline 510.01 & $0.02599 \pm 0.00166$ & $-\cdots$ & $0.01749 \pm 0.00048$ \\
\hline 520.01 & $0.02480 \pm 0.00168$ & $m-r$ & $0.01630 \pm 0.00053$ \\
\hline 530.01 & $0.02314 \pm 0.00167$ & $-=-$ & $0.01464 \pm 0.00051$ \\
\hline 540.01 & $0.02111 \pm 0.00167$ & $-m$ & $0.01261 \pm 0.00050$ \\
\hline 550.01 & $0.01921 \pm 0.00168$ & r- & $0.01071 \pm 0.00053$ \\
\hline 560.01 & $0.01754 \pm 0.00166$ & $-+\infty$ & $0.00904 \pm 0.00048$ \\
\hline 570.01 & $0.01607 \pm 0.00168$ & $--\infty$ & $0.00757 \pm 0.00053$ \\
\hline 580.01 & $0.01472 \pm 0.00167$ & $-\infty$ & $0.00622 \pm 0.00051$ \\
\hline 590.01 & $0.01346 \pm 0.00167$ & $m-$ & $0.00496 \pm 0.00050$ \\
\hline 600.01 & $0.01240 \pm 0.00168$ & $-\infty$ & $0.00390 \pm 0.00054$ \\
\hline 610.01 & $0.01155 \pm 0.00166$ & $m-$ & $0.00305 \pm 0.00049$ \\
\hline 620.01 & $0.01080 \pm 0.00168$ & $-\infty$ & $0.00230 \pm 0.00054$ \\
\hline 630.01 & $0.00993 \pm 0.00167$ & 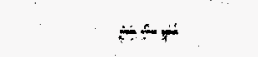 & $0.00143 \pm 0.00052$ \\
\hline 640.01 & $0.00896 \pm 0.00169$ & $-\infty$ & $0.00046 \pm 0.00057$ \\
\hline 650.01 & $0.00850 \pm 0.00168$ & $\rightarrow-r$ & $0.00000 \pm 0.00053$ \\
\hline
\end{tabular}


TABLE 11.6 Differential Reactivity Worth, in Cents per Kilogram, of Sodium Column in 149-40

\begin{tabular}{|c|c|c|c|}
\hline $\begin{array}{l}\text { Distance from } \\
\text { Interface (mm) }\end{array}$ & $\begin{array}{c}\text { Corrected, } \\
\text { Adjusted } \\
\end{array}$ & $\begin{array}{c}\text { Worth Above } \\
660 \mathrm{~mm} \\
\end{array}$ & Uncorrected \\
\hline 2.31 & $-0.5883 \pm 0.0621$ & $-0.5241 \pm 0.0491$ & $-1.1124 \pm 0.0381$ \\
\hline 10.00 & $-0.5669 \pm 0.0643$ & $-0.4050 \pm 0.0508$ & $-0.9719 \pm 0.0395$ \\
\hline 20.00 & $\therefore 0.5391 \pm 0.0631$ & $-0.2500 \pm 0.0499$ & $-0.7891 \pm 0.0387$ \\
\hline 30.00 & $-0.5327 \pm 0.0604$ & $-0.1241 \pm 0.0477$ & $-0.6568 \pm 0.0371$ \\
\hline 40.00 & $-0.5984 \pm 0.0610$ & $-0.0960 \pm 0.0482$ & $-0.6944 \pm 0.0374$ \\
\hline 50.00 & $-0.6641 \pm 0.0579$ & $-0.0678 \pm 0.0458$ & $-0.7319 \pm 0.0355$ \\
\hline 60.00 & $-0.6828 \pm 0.0614$ & $-0.0215 \pm 0.0485$ & $-0.7043 \pm 0.0377$ \\
\hline 70.00 & $-0.6804 \pm 0.0576$ & $0.0331 \pm 0.0455$ & $-0.6473 \pm 0.0354$ \\
\hline 80.00 & $-0.6659 \pm 0.0586$ & $0.0668 \pm 0.0463$ & $-0.5991 \pm 0.0360$ \\
\hline 90.00 & $-0.5834 \pm 0.0601$ & $-0.0179 \pm 0.0475$ & $-0.6013 \pm 0.0369$ \\
\hline 100.00 & $-0.5010 \pm 0.0571$ & $-0.1025 \pm 0.0451$ & $-0.6035 \pm 0.0350$ \\
\hline 110.00 & $-0.4883 \pm 0.0610$ & $-0.1298 \pm 0.0482$ & $-0.6181 \pm 0.0374$ \\
\hline 120.00 & $-0.5206 \pm 0.0576$ & $-0.1198 \pm 0.0455$ & $-0.6404 \pm 0.0353$ \\
\hline 130.00 & $-0.5463 \pm 0.0582$ & $-0.1158 \pm 0.0460$ & $-0.6621 \pm 0.0357$ \\
\hline 140.00 & $-0.4835 \pm 0.0604$ & $-0.1898 \pm 0.0477$ & $-0.6733 \pm 0.0371$ \\
\hline 150.00 & $-0.4208 \pm 0.0571$ & $-0.2638 \pm 0.0451$ & $-0.6846 \pm 0.0350$ \\
\hline 160.00 & $-0.4363 \pm 0.0606$ & $-0.1659 \pm 0.0479$ & $-0.6022 \pm 0.0372$ \\
\hline 170.00 & $-0.5215 \pm 0.0577$ & $0.0852 \pm 0.0456$ & $-0.4363 \pm 0.0354$ \\
\hline 180.00 & $-0.6068 \pm 0.0573$ & $0.3363 \pm 0.0454$ & $-0.2705 \pm 0.0350$ \\
\hline 190.00 & $-0.5537 \pm 0.0602$ & $0.2088 \pm 0.0476$ & $-0.3449 \pm 0.0368$ \\
\hline 200.00 & $-0.4992 \pm 0.0565$ & $0.0772 \pm 0.0447$ & $-0.4220 \pm 0.0346$ \\
\hline 210.00 & $-0.4386 \pm 0.0597$ & $-0.0058 \pm 0.0473$ & $-0.4444 \pm 0.0365$ \\
\hline 220.00 & $-0.3705 \pm 0.0577$ & $-0.0293 \pm 0.0457$ & $-0.3998 \pm 0.0353$ \\
\hline 230.00 & $-0.3024 \pm 0.0570$ & $-0.0528 \pm 0.0451$ & $-0.3552 \pm 0.0348$ \\
\hline 240.00 & $-0.2762 \pm 0.0605$ & $-0.0631 \pm 0.0479$ & $-0.3393 \pm 0.0369$ \\
\hline 250.00 & $-0.2543 \pm 0.0568$ & $-0.0720 \pm 0.0451$ & $-0.3263 \pm 0.0345$ \\
\hline 260.00 & $-0.2436 \pm 0.0599$ & $-0.0431 \pm 0.0476$ & $-0.2867 \pm 0.0363$ \\
\hline 270.00 & $-0.2521 \pm 0.0589$ & $0.0504 \pm 0.0469$ & $-0.2017 \pm 0.0356$ \\
\hline 280.00 & $-0.2608 \pm 0.0591$ & $0.1441 \pm 0.0479$ & $-0.1167 \pm 0.0346$ \\
\hline 290.00 & $-0.1766 \pm 0.0641$ & $0.0013 \pm 0.0524$ & $-0.1753 \pm 0.0369$ \\
\hline 300.00 & $-0.0734 \pm 0.0613$ & $-0.1900 \pm 0.0507$ & $-0.2634 \pm 0.0345$ \\
\hline 310.00 & $-0.2881 \pm 0.0360$ & $-\infty$ & $-0.2881 \pm 0.0360$ \\
\hline 320.00 & $-0.1567 \pm 0.0358$ & $\ldots-$ & $-0.1567 \pm 0.0358$ \\
\hline 330.00 & $-0.0253 \pm 0.0345$ & $-\infty$ & $-0.0253 \pm 0.0345$ \\
\hline 340.00 & $0.0277 \pm 0.0369$ & $m-$ & $0.0277 \pm 0.0369$ \\
\hline 350.00 & $0.0544 \pm 0.0346$ & -- & $0.0544 \pm 0.0346$ \\
\hline 360.00 & $0.0630 \pm 0.0356$ & $-\cdots-$ & $0.0630 \pm 0.0356$ \\
\hline 370.00 & $0.0032 \pm 0.0361$ & -- & $0.0032 \pm 0.0361$ \\
\hline 380.00 & $-0.0565 \pm 0.0345$ & $-\cdots$ & $-0.0565 \pm 0.0345$ \\
\hline 390.00 & $0.0093 \pm 0.0369$ & $-\cdots$ & $0.0093 \pm 0.0369$ \\
\hline 400.00 & $0.1373 \pm 0.0346$ & $m-$ & $0.1373 \pm 0.0346$ \\
\hline 410.00 & $0.2352 \pm 0.0353$ & $-\infty$ & $0.2352 \pm 0.0353$ \\
\hline 420.00 & $0.1314 \pm 0.0363$ & -- & $0.1314 \pm 0.0363$ \\
\hline 430.00 & $0.0275 \pm 0.0345$ & $-m$ & $0.0275 \pm 0.0345$ \\
\hline 440.00 & $0.0517 \pm 0.0368$ & $-\infty$ & $0.0517 \pm 0.0368$ \\
\hline 450.00 & $0.1650 \pm 0.0348$ & $-\cdots$ & $0.1650 \pm 0.0348$ \\
\hline 460.01 & $0.2754 \pm 0.0351$ & - & $0.2754 \pm 0.0351$ \\
\hline 470.01 & $0.3267 \pm 0.0366$ & $-\infty$ & $0.3267 \pm 0.0366$ \\
\hline 480.01 & $0.3780 \pm 0.0345$ & $m-\infty$ & $0.3780 \pm 0.0345$ \\
\hline 490.01 & $0.3586 \pm 0.0366$ & $-\cdots$ & $0.3586 \pm 0.0366$ \\
\hline
\end{tabular}


TABLE 11.6 (Cont $d$ )

\begin{tabular}{|c|c|c|c|}
\hline $\begin{array}{l}\text { Distance from } \\
\text { Interface (mm) }\end{array}$ & $\begin{array}{c}\text { Corrected, } \\
\text { Adjusted }\end{array}$ & $\begin{array}{l}\text { Worth Above } \\
660 \mathrm{~mm} \\
\end{array}$ & Uncorrected \\
\hline 500.01 & $0.2709 \pm 0.0350$ & $-m$ & $0.2709 \pm 0.0350$ \\
\hline 510.01 & $0.1831 \pm 0.0348$ & $\cdots-$ & $0.1831 \pm 0.0348$ \\
\hline 520.01 & $0.2617 \pm 0.0368$ & $-\cdots$ & $0.2617 \pm 0.0368$ \\
\hline 530.01 & $0.3454 \pm 0.0345$ & $\ldots$ & $0.3454 \pm 0.0345$ \\
\hline 540.01 & $0.3737 \pm 0.0364$ & $\ldots$ & $0.3737 \pm 0.0364$ \\
\hline 550.01 & $0.3285 \pm 0.0353$ & $\cdots-$ & $0.3285 \pm 0.0353$ \\
\hline 560.01 & $0.2833 \pm 0.0348$ & $\ldots$ & $0.2833 \pm 0.0348$ \\
\hline 570.01 & $0.2607 \pm 0.0369$ & $\cdots$ & $0.2607 \pm 0.0369$ \\
\hline 580.01 & $0.2408 \pm 0.0346$ & $\ldots$ & $0.2408 \pm 0.0346$ \\
\hline 590.01 & $0.2146 \pm 0.0365$ & $\ldots$ & $0.2146 \pm 0.0365$ \\
\hline 600.01 & $0.1763 \pm 0.0358$ & $-\cdots$ & $0.1763 \pm 0.0358$ \\
\hline 610.01 & $0.1379 \pm 0.0361$ & $\therefore-$ & $0.1379 \pm 0.0361$ \\
\hline 620.01 & $0.1493 \pm 0.0382$ & $-\cdots$ & $0.1493 \pm 0.0382$ \\
\hline 630.01 & $0.1724 \pm 0.0405$ & $\ldots-$ & $0.1724 \pm 0.0405$ \\
\hline 640.01 & $0.1526 \pm 0.0415$ & $-\cdots$ & $0.1526 \pm 0.0415$ \\
\hline 650.01 & $0.0160 \pm 0.0415$ & $\ldots-\infty$ & $0.0160 \pm 0.0415$ \\
\hline
\end{tabular}




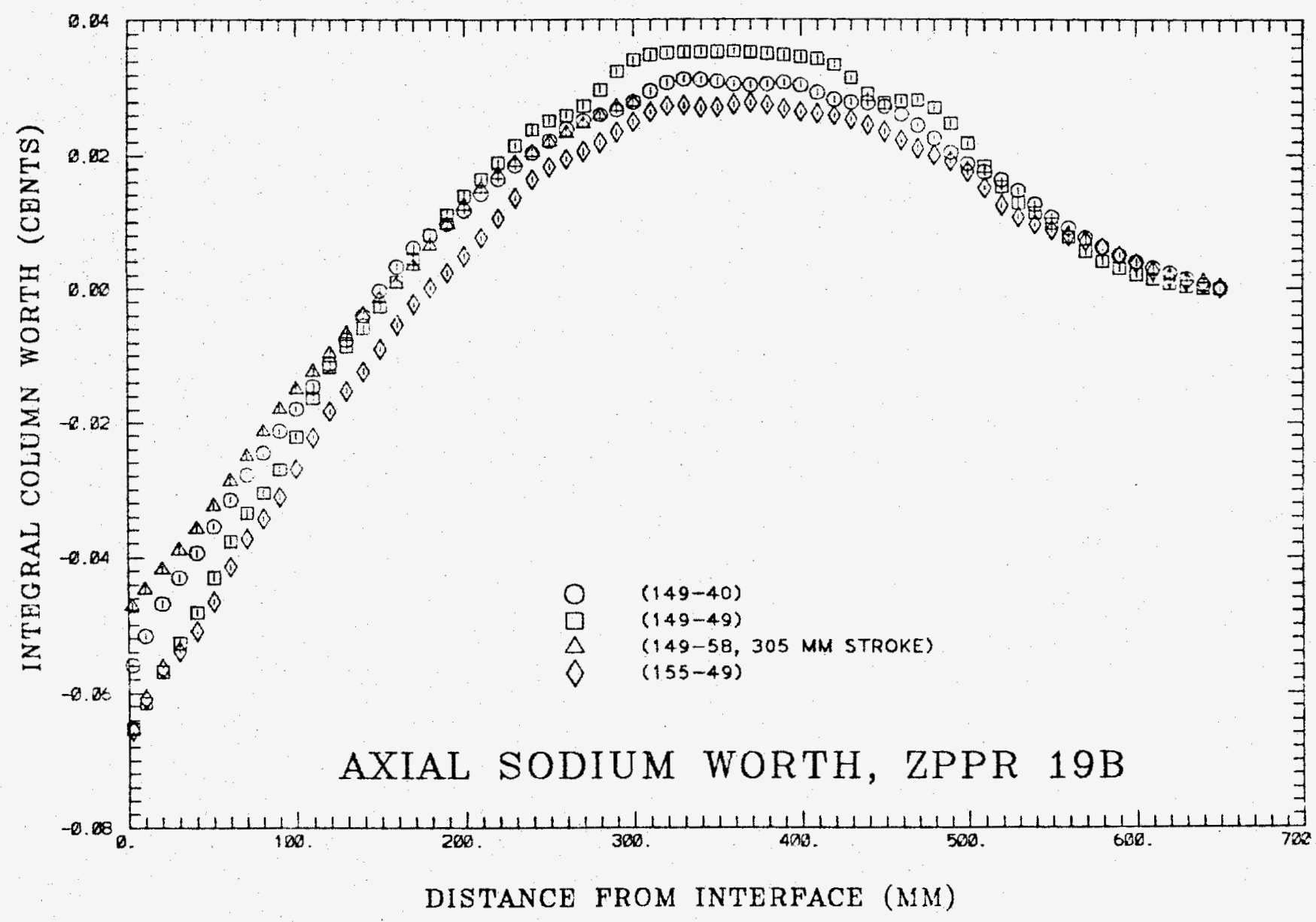

Eig. 11.1 Integral Sodium Worth Axial Profiles in ZPPR-19B 
DIFFERENTIAL WORTH (CENTS/KG.)

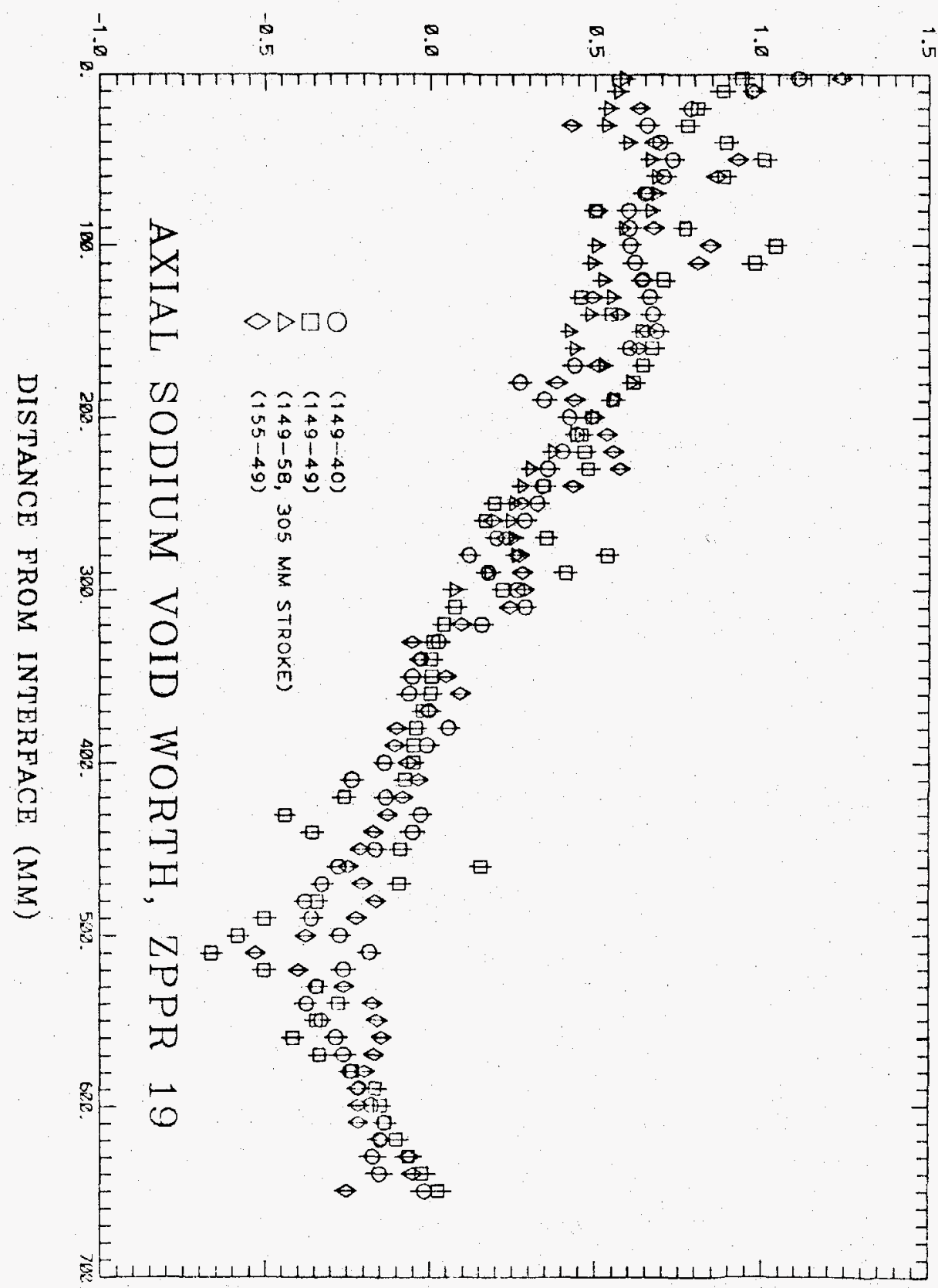




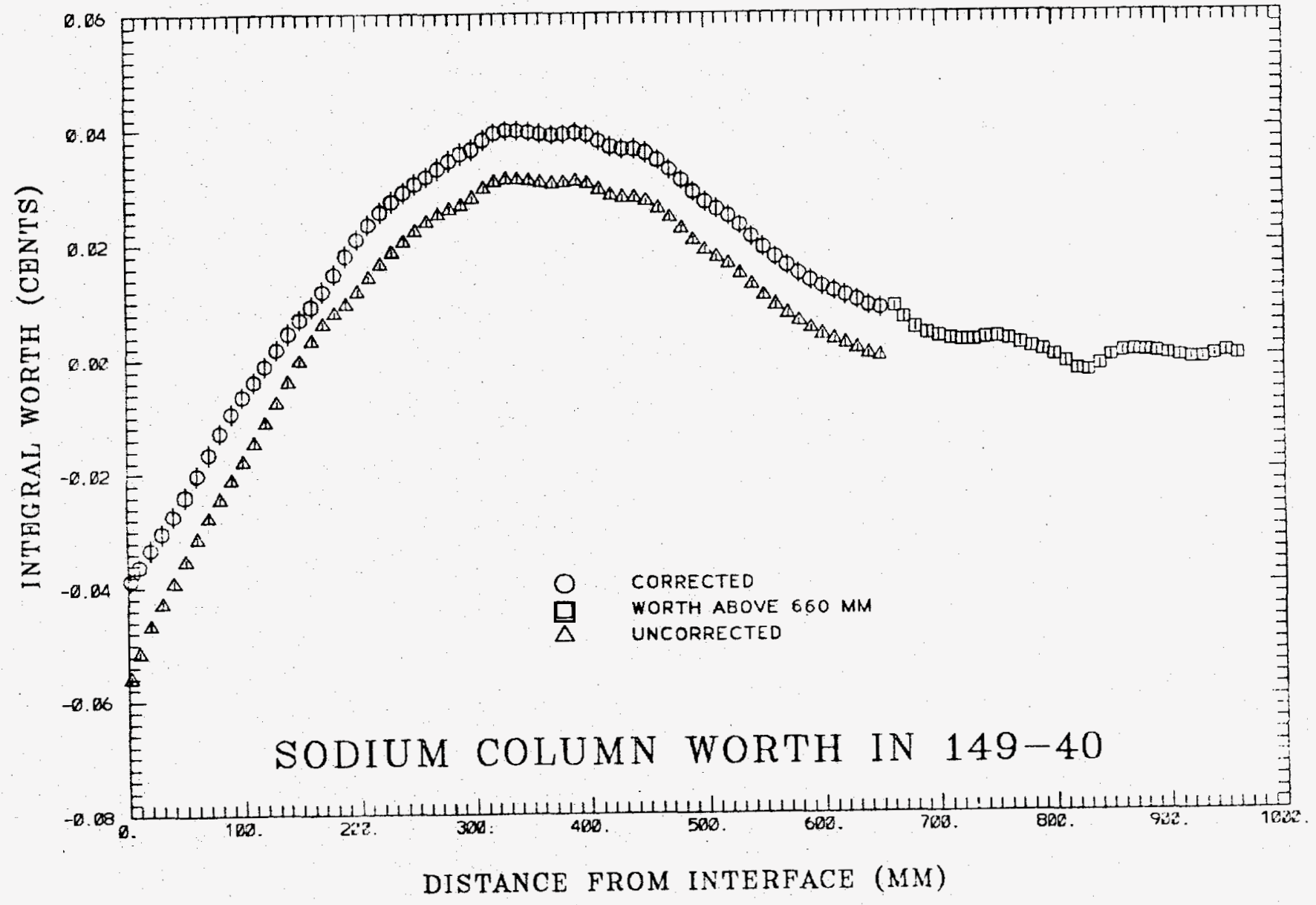

Fig. 11.3 Comparison of Corrected and Uncorrected Integral Sodium Worth Axial Profiles in ZPPR -19B 


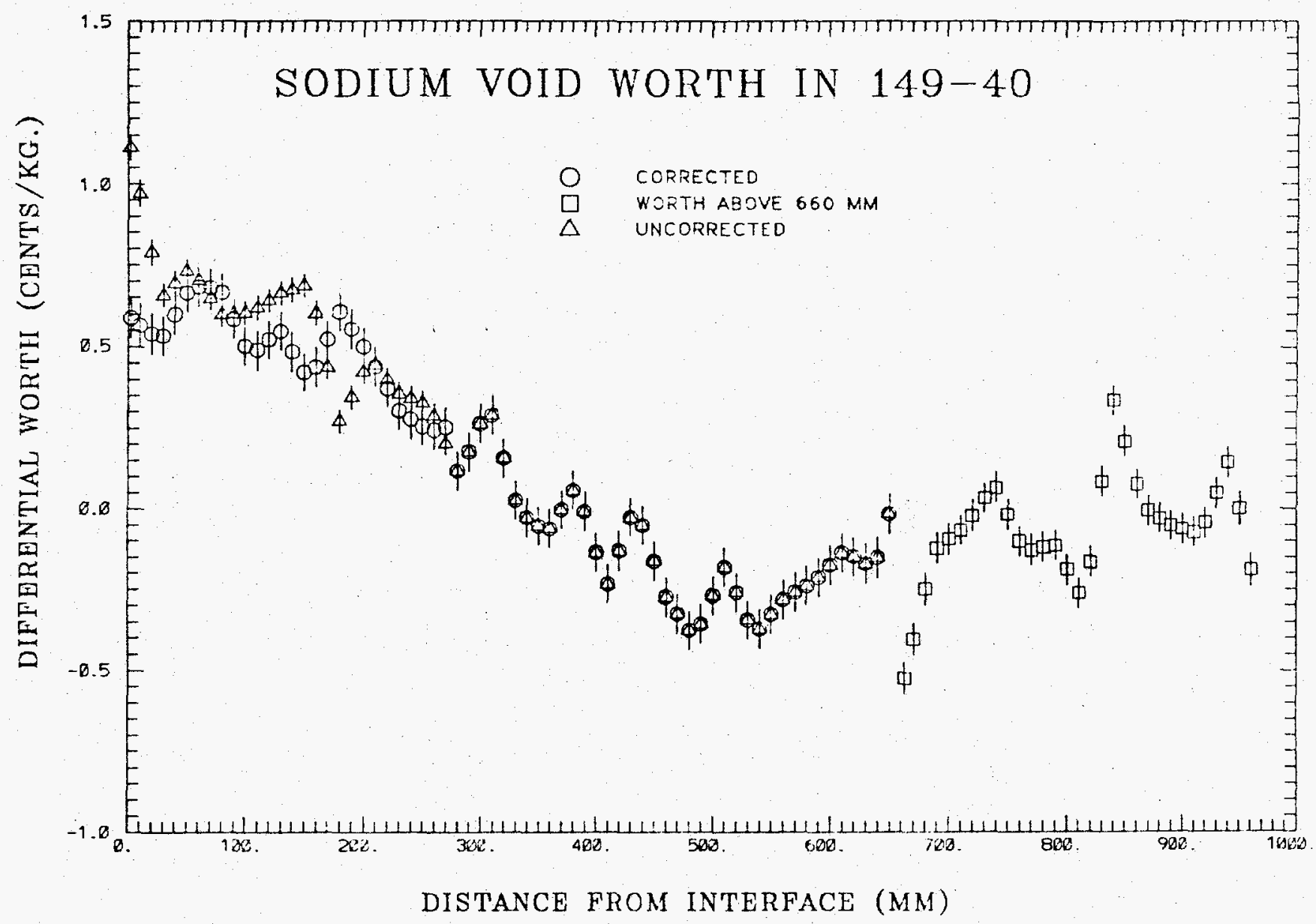

Fig. 11.4 Comparison of Corrected and Uncorrected Differential Sodium Worth Axial Profiles in ZPPR-19B 
12. $\frac{\text { ON THE MODELING OF THE URANIUM FUEL IN ZPPR-18 }}{\text { (G. L. Grasseschi and P. J. Collins) }}$

The axial half-height of the uranium fuel in $Z P P R-18$ was $1.11 \mathrm{~cm}$ greater than that of the plutonium fuel. To avoid using a fine node spacing in the nodal transport calculations, the xyz model for ZPPR- 18 smeared the extra $1.11 \mathrm{~cm}$ of uranium over the first $5.08 \mathrm{~cm}$ node of the axial blanket (ANL-ZPR-489, p. 13). In order to test the effect of this approximation, two calculations were made using finite-difference diffusion theory (FDDT) with mesh boundaries 1.11 and $5.08 \mathrm{~cm}$ above the axial blanket boundary. The first FDDT calculation smeared the uranium through $5.08 \mathrm{~cm}$ as in the nodal calculation. The second FDDT calculation retained the uranium in the $1.11 \mathrm{~cm}$ region only. In the plutonium sector, the $1.11 \mathrm{~cm}$ region was assigned only axial blanket material.

The differences between the FDDT model and the reference model appear to be negligible for most parameters calculated in ZPPR 18:

i. k-effective differed by 0.000001

if Reaction rate distributions near the midplane and at $28 \mathrm{~cm}$ from the midplane differed by less than $0.1 \%$

ii $i$ The axial reaction rate distributions in the outer core at the $x^{-}$ axis differed by less than $0.1 \%$, including values in the axial blanket. (Only a very small difference in blanket compositions existed in these locations due to different piece lengths between the first $1.11 \mathrm{~cm}$ and the entire blanket thickness.)

Differences for a ${ }^{235} \mathrm{U}$ fission distribution in location 121-49, along the $y$-axis in the uranium sector, are shown in Table 12.1. A difference of $0.9 \%$ is indicated at the inner edge of the blanket which changes the source of neutrons into the blanket and reflection of neutron back to the core. Some uncertainty in these results may exist due to mesh-size effects.

Calculated control rod worths for outer ring rods at the boundary of the uranium sector may be affected by a few tenths of a percent by the simplified modeling. In ZPPR-19B the axial distribution in the outer core 
of mixed uranium and plutonium drawers will also be changed but these effects will be masked by experimental statistics and other modeling approximations. Note that, on a finer scale, all ZPPR models have smeared fuel over the full lengths of the fuel column and neglected effects of the end clads. 
TABLE 12.1 Comparison of ${ }^{235} \mathrm{U}$ Fission Rates in the Uranium Sector

\begin{tabular}{|c|c|c|c|c|}
\hline \multirow[b]{2}{*}{ Zone } & \multirow{2}{*}{$\begin{array}{l}\text { Mesh Boundary, } \\
\text { Distance from } \\
\text { Midplane, cm }\end{array}$} & \multicolumn{3}{|c|}{ Mesh-average Fission Rate } \\
\hline & & $\begin{array}{l}\text { Refined } \\
\text { Model } \\
\end{array}$ & $\begin{array}{c}\text { Reference } \\
\text { Model } \\
\end{array}$ & Ratio \\
\hline \multirow[t]{5}{*}{ Core } & 36.80 & & & 00008 \\
\hline & 41.49 & יכל & בנם (19) & של \\
\hline & 46.18 & 10.400 & 10.409 & 0.9991 \\
\hline & & 9.0000 & 9.0204 & 0.9973 \\
\hline & 50.88 & 8.2819 & 8.3003 & 0.9978 \\
\hline Uranium & 51.99 & 8.6056 & 8.5258 & 1.0094 \\
\hline \multirow[t]{6}{*}{$\begin{array}{l}\text { Axial } \\
\text { Blanket }\end{array}$} & 55.96 & 7.8712 & 7.8181 & 1.0068 \\
\hline & 60.53 & 6.9980 & 6.9634 & 1.0050 \\
\hline & 65.10 & & י & 1.000 \\
\hline & 69.67 & 6.1448 & 6.1229 & 1.0036 \\
\hline & 74.24 & 5.4114 & 5.3973 & 1.0060 \\
\hline & & 4.9089 & 4.8988 & 1.0021 \\
\hline Reflector & 78.82 & & & \\
\hline
\end{tabular}

${ }^{a}$ Abritrary units, both calculations normalized to the same reactor power. 